Marcelo Tanaka Hayashi

\title{
CÁlCulo de SENSIBILIDADES NÃO-GEOMÉTRICAS EM ESCOAMENTOS MODELADOS PELAS EQUAÇÕES DE EULER COMPRESSÍVEIS UTILIZANDO O MÉTODO ADJUNTO
}

Tese apresentada à Escola Politécnica da Universidade de São Paulo para obtenção do Título de Doutor em Engenharia Mecânica. 
Marcelo Tanaka Hayashi

\section{CÁlCulo de SENSIBILIDADES NÃO-GEOMÉTRICAS EM ESCOAMENTOS MODELADOS PELAS EQUAÇÕES DE EULER COMPRESSÍVEIS UTILIZANDO O MÉTODO ADJUNTO}

Tese apresentada à Escola Politécnica da Universidade de São Paulo para obtenção do Título de Doutor em Engenharia Mecânica.

Área de concentração:

Engenharia Mecânica

Orientador:

Prof. Dr. Ernani Vitillo Volpe 
Este exemplar foi revisado e corrigido em relação à versão original, sob responsabilidade única do autor e com a anuência de seu orientador.

São Paulo, de de

Assinatura do autor:

Assinatura do orientador:

\section{Catalogação-na-publicação}

Hayashi, Marcelo Tanaka

Cálculo de sensibilidades não-geométricas em escoamentos modelados pelas equações de Euler compressíveis utilizando o método adjunto / M. T. Hayashi -- versão corr. -- São Paulo, 2016. $113 \mathrm{p}$.

Tese (Doutorado) - Escola Politécnica da Universidade de São Paulo. Departamento de Engenharia Mecânica.

1.Método adjunto 2.Sensibilidades não-geométricas 3.Aerodinâmica 4.Dinâmica dos fluidos computacional 5.Condições de contorno I.Universidade de São Paulo. Escola Politécnica. Departamento de Engenharia Mecânica II.t. 


\section{DEDICATÓRIA}

Aos meus pais e irmã, que jamais pouparam esforços em prol da minha educação. 


\section{AgRADECIMENTOS}

Em primeiro lugar, gostaria de agradecer ao Prof. Dr. Ernani Vitillo Volpe pela orientação, suporte e, sobretudo, por ter me dado a oportunidade de trabalhar ao seu lado durante todos estes anos dedicados às atividades de pesquisa.

Ao Conselho Nacional de Desenvolvimento Científico e Tecnológico (CNPq) que, através da concessão da bolsa de estudos, propiciou a minha dedicação integral para o desenvolvimento desta tese.

Ao pesquisador do Instituto de Pesquisas Tecnológicas (IPT) Dr. Paulo Jabardo que, durante um momento de dificuldade no andamento do trabalho, contribuiu com sugestões sobre a utilização de ferramentas computacionais que se mostraram fundamentais para a obtenção dos resultados aqui apresentados.

Ao Prof. Dr. João Azevedo (ITA), com quem aprendi toda a fundamentação teórica a respeito de métodos numéricos para escoamentos compressíveis e que utilizei durante toda a minha vida acadêmica como pós-graduando. Além disso, agradeço pelo constante acompanhamento do meu trabalho realizado em poucas, mas proveitosas reuniões ao longo dos úlimos anos.

De uma maneira geral, agradeço a todos os professores que tive até hoje. Entretanto, não posso deixar de citar nomes como: Prof. Dr. José Augusto Penteado Aranha (EPUSP), Prof. Dr. Bruno Carmo (EPUSP), Prof. Dr. Roberto Gil (ITA), Prof. Dr. Marcos Pimenta (EPUSP) e Prof. Dr. Rafael Giória (EPUSP) pelas correções e valiosas sugestões que resultaram na edição revisada desta tese.

Aos membros do Núcleo de Dinâmica dos Fluidos (NDF-USP), em especial aos que participaram em algum momento do grupo de pesquisa a que pertenço: Dr. Marco Ceze e os doutorandos Bruno Chieregatti e João Brasil.

Por fim, não posso deixar de agradecer aos meus amigos que por razões diferentes, ainda que indiretamente, contribuiram para a conclusão deste trabalho: Andrei Agostinho, Daniela Muniz, Erika Carvalho, Fabiana Rabacow, Janilton Gomes, Letícia Vicente, Mariana Madruga e Thales Albuquerque. 
"Um especialista é alguém que cometeu todos os erros possiveis em um campo do conhecimento muito restrito."

Niels Henrik David Bohr 


\section{RESUMO}

O método adjunto tem sido extensivamente utilizado como ferramenta de síntese no projeto de aeronaves por permitir que se obtenham sensibilidades de distintas medidas de mérito com relação a parâmetros que controlam a geometria de superfícies aerodinâmicas. O presente trabalho visa uma ampliação das aplicações da formulação contínua do método, ao utilizar propriedades físicas do escoamento nas fronteiras permeáveis do domínio computacional como parâmetros de controle de uma particular medida de mérito. Desse modo é possível, entre muitas possibilidades, determinar a sensibilidade de integrais como sustentação ou arrasto de uma aeronave com relação às condições de cruzeiro, por exemplo. Mais do que isso, essa informação pode ser obtida com a mesma solução adjunta computada para realizar otimização de forma. Vale destacar, ainda, que para que se consiga obter essa informação a partir das equações adjuntas, é necessário que se implemente condições de contorno baseadas em equações diferenciais características, resolvendo o problema de Riemann completo nas fronteiras do domínio. A implementação das usuais condições de contorno homogêneas, vastamente difundidas na literatura, resultaria em gradientes nulos. Esta nova abordagem do método é então aplicada a escoamentos modelados pelas equações de Euler 2-D compressíveis em estado estacionário. Ambos os problemas, físico e adjunto, são resolvidos numericamente com um código computacional que utiliza o método dos volumes finitos com segunda ordem de precisão no espaço e discretização centrada com dissipação artificial. As soluções estacionárias são obtidas ao se postular um termo tempo-dependente e integrá-lo com um esquema Runge-Kutta de 5 passos e $2^{\text {a }}$ ordem de precisão. As simulações são realizadas em malhas nãoestruturadas formadas por elementos triangulares em 4 geometrias distintas: um bocal divergente, um perfil diamante, um aerofólio simétrico (NACA 0012) e o outro assimétrico (RAE 2822). Os gradientes adjuntos são então validados por meio da comparação com os obtidos pelo método de diferenças finitas nos regimes de escoamento subsônico, supersônico e transônico.

Palavras chave: Método Adjunto, Otimização, Aerodinâmica, Condições de Contorno. 


\section{ABSTRACT}

The adjoint method has been extensively used as an aircraft design tool, since it enables one to obtain sensitivities of many different mesures of merit with respect to parameters that control the aerodynamic surface geometry. This works aims to open up the possibilities of the method's applications by using flow physical properties at the permeable boundaries of the computational domain as control parameters of a particular measure of merit. This way it is possible, among many possibilities, to compute lift or drag sensitivities of an aircraft with respect to cruise conditions, for instance. Moreover, this information can be obtained with the same adjoint solution used to perform shape optimization. It is also worth noting that in order to obtain this information from the adjoint equations it is necessary to implement characteristics-based boundary conditions, resolving the complete Riemann problem at the boundaries of the computational domain. The use of the traditional homogeneous boundary conditions, widely spread in the literature, would lead the gradient to vanish. This new approach of the method is, then, applied to flows modeled by the 2-D steady state compressible Euler equations. Both, physical and adjoint problems are numerically solved with a computational code that makes use of a $2^{\text {nd }}$ order finite volume method and central differences with artifficial dissipation. The steady solutions are obtained by postulating a time-dependent term and integrating it with a 5-stage $2^{\text {nd }}$ order Runge-Kutta scheme. The simulations are performed on unstructured triangular meshes to 4 different geometries: a divergent nozzle, a diamond profile, a symmetric airfoil (NACA 0012) and a assymmetric airfoil (RAE 2822). The adjoint gradients are then validated by comparison with those obtained by finite differences method in subsonic, supersonic and transonic flow regimes.

Key words: Adjoint Method, Optimization, Aerodynamics, Boundary Conditions. 


\title{
SUMÁRIO
}

\section{Lista de Figuras}

\author{
Lista de Tabelas
}

\section{Acrônimos}

\section{Nomenclatura}

1 Introdução 1

1.1 O Projeto Aerodinâmico . . . . . . . . . . . . . . . . . . . . 1

1.2 Métodos de Otimização e Projeto Inverso . . . . . . . . . . . . . . 2

1.3 O Método Adjunto e Suas Aplicações . . . . . . . . . . . . . . . . 4

1.4 Formulação do Problema Adjunto . . . . . . . . . . . . . . . . . . 6

1.4.1 Formas de Discretização da Equação Adjunta .... . 7

1.4.2 Abordagem Tradicional - Jameson . . . . . . . . . . . 8

1.4.3 Abordagem Alternativa - Cacuci . . . . . . . . . . . . 14

1.5 Histórico de Pesquisas . . . . . . . . . . . . . . . . . . 17

1.6 Objetivos, Contribuição e Estrutura da Tese . . . . . . . . . . . . 20

2 Modelo Matemático do Escoamento 22

2.1 As Equações de Navier-Stokes . . . . . . . . . . . . . . . . . . . 22

2.2 As Equações de Euler . . . . . . . . . . . . . . . . . . . . . . . . 23

2.2.1 Forma Característica das Equações de Euler . . . . . . . . 26

2.2.2 Equações de Euler em Coordenadas Generalizadas . . . . . 32

2.2.3 Representação Utilizando Notação Indicial . . . . . . . . . 37 
3.1 O Problema Variacional . . . . . . . . . . . . . . . . . . . 39

3.2 As Equações Adjuntas . . . . . . . . . . . . . . . . . . . . . 43

3.3 Condições de Contorno Adjuntas . . . . . . . . . . . . . . . . . . 44

3.3.1 O Problema 2-D ................... 45

3.4 Gradiente Adjunto . . . . . . . . . . . . . . . . 59

4 Resultados $\quad 61$

4.1 Método de Validação da Solução Adjunta . . . . . . . . . . . . . . 61

4.2 Medidas de Mérito Adotadas . . . . . . . . . . . . . . . . . . 62

4.3 Escoamento Interno . . . . . . . . . . . . . . . . . . 63

4.3 .1 Caso Supersônico . . . . . . . . . . . . . . . . . 64

4.3 .2 Caso Subsônico . . . . . . . . . . . . . . . . 66

4.4 Escoamento Externo . . . . . . . . . . . . . . . 69

$4.4 .1 \quad$ Perfil Diamante . . . . . . . . . . . . . . . 69

$4.4 .2 \quad$ Perfil RAE $2822 \ldots \ldots \ldots \ldots$. . . . . . . . . . . 79

4.4 .3 Perfil NACA $0012 \ldots \ldots \ldots \ldots . \ldots \ldots$

5 Conclusões e Trabalhos Futuros $\quad 99$

5.1 Conclusões . . . . . . . . . . . . . . . . . . . . . . . . 99

5.2 Trabalhos Futuros . . . . . . . . . . . . . . . . 100

$\begin{array}{ll}\text { Referências } & 102\end{array}$

Apêndice A - Coeficientes das Equações Características Adjuntas 109

Apêndice B - Redução do Gradiente Geométrico 111

Apêndice C - Diagrama $\theta-\beta-M \quad 112$ 


\section{LisTA DE FiguRAS}

1.1 Teste de validação de projeto inverso aerodinâmico $\left(M_{\infty}=0.75\right.$,

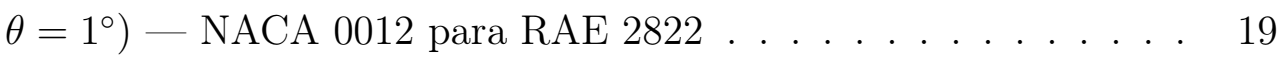

1.2 Exemplo de aplicação de projeto inverso aerodinâmico $\left(M_{\infty}=\right.$ $\left.0.75, \theta=1^{\circ}\right) \ldots \ldots \ldots \ldots \ldots$

2.1 Transformação de coordenadas . . . . . . . . . . . . . . . . . . . 32

3.1 Propagação de características adjuntas em fronteiras de saída . . . 48

3.2 Propagação de características adjuntas em fronteiras de entrada . 52

4.1 Escoamento supersônico em bocal divergente $(M=2) . \ldots .64$

4.2 Solução adjunta em bocal divergente supersônico $(M=2, I=L) . \quad 65$

4.3 Sensibilidade em bocal supersônico . . . . . . . . . . . . . . 65

4.4 Diferenças de sensibilidades em bocal supersônico . . . . . . . . . 66

4.5 Históricos de convergência dos problemas físico e adjunto em bocal divergente supersônico. . . . . . . . . . . . . . . . . 66

4.6 Sensibilidade em bocal subsônico . . . . . . . . . . . . . . . 67

4.7 Diferenças de sensibilidades em bocal subsônico (Adjunto vs. Diferenças Finitas) . . . . . . . . . . . . . . . . . . . . . 68

4.8 Históricos de convergência dos problemas físico e adjunto em bocal divergente subsônico. . . . . . . . . . . . . . . . . . 68

4.9 Malhas tipicamente utilizadas para simular escoamentos externos em torno de aerofólios. . . . . . . . . . . . . . . . . . . . . 69

4.10 Malha com condições de contorno periódicas nas fronteiras superior e inferior. . . . . . . . . . . . . . . . . . 70

4.11 Escoamento supersônico em perfil diamante $\left(M_{\infty}=1.8, \theta=2^{\circ}\right) \quad . \quad 70$

4.12 Solução adjunta de escoamento supersônico em perfil diamante $\left(M_{\infty}=1.8, \theta=2^{\circ}\right) \ldots \ldots \ldots \ldots \ldots \ldots \ldots$ 
4.13 Sensibilidade da sustentação em perfil diamante $\operatorname{com} \theta=0^{\circ} \quad$. . 72

4.14 Sensibilidade da sustentação em perfil diamante $\operatorname{com} \theta=1^{\circ} \quad \ldots \quad 72$

4.15 Sensibilidade da sustentação em perfil diamante $\operatorname{com} \theta=2^{\circ} \quad \ldots \quad 72$

4.16 Sensibilidade da sustentação em perfil diamante $\operatorname{com} \theta=3^{\circ} \ldots 73$

4.17 Sensibilidade da sustentação em perfil diamante $\operatorname{com} \theta=4^{\circ} \quad \ldots \quad 73$

4.18 Históricos de convergência dos problemas físico e adjunto em perfil diamante supersônico. . . . . . . . . . . . . . . . . . 74

4.19 Perfil diamante $-\partial L / \partial \rho \ldots \ldots \ldots \ldots . \ldots . \ldots . \ldots 74$

4.20 Perfil diamante $-\partial L / \partial u \ldots \ldots \ldots . \ldots . \ldots . \ldots 74$

4.21 Perfil diamante $-\partial L / \partial v \ldots \ldots \ldots \ldots \ldots . \ldots . \ldots . \ldots 75$

4.22 Perfil diamante $-\partial L / \partial p \ldots \ldots \ldots \ldots . \ldots . \ldots 75$

4.23 Sensibilidade do arrasto de onda em perfil diamante $\operatorname{com} \theta=0^{\circ} \quad . \quad 76$

4.24 Sensibilidade do arrasto de onda em perfil diamante $\operatorname{com} \theta=1^{\circ} \quad . \quad 76$

4.25 Sensibilidade do arrasto de onda em perfil diamante $\operatorname{com} \theta=2^{\circ} \quad . \quad 76$

4.26 Sensibilidade do arrasto de onda em perfil diamante $\operatorname{com} \theta=3^{\circ} \quad . \quad 77$

4.27 Sensibilidade do arrasto de onda em perfil diamante $\operatorname{com} \theta=4^{\circ} \quad . \quad 77$

4.28 Perfil diamante $-\partial D / \partial \rho \ldots \ldots \ldots \ldots 77$

4.29 Perfil diamante $-\partial D / \partial u \ldots \ldots \ldots \ldots 78 \ldots \ldots$

4.30 Perfil diamante $-\partial D / \partial v \ldots \ldots \ldots \ldots . \ldots . \ldots 78$

4.31 Perfil diamante $-\partial D / \partial p \ldots \ldots \ldots \ldots . \ldots . \ldots . \ldots 78$

4.32 Solução do perfil RAE $2822\left(M_{\infty}=0.9, \theta=0^{\circ}\right) \ldots \ldots$

4.33 Solução adjunta do perfil RAE $2822\left(M_{\infty}=0.9, \theta=0^{\circ}\right) \quad \ldots \quad . \quad 80$

4.34 Sensibilidade da sustentação em perfil RAE $2822 \operatorname{com} \theta=0^{\circ} \ldots 81$

4.35 Sensibilidade da sustentação em perfil RAE $2822 \operatorname{com} \theta=1^{\circ} \ldots 81$

4.36 Sensibilidade da sustentação em perfil RAE $2822 \operatorname{com} \theta=2^{\circ} \ldots 81$

4.37 Sensibilidade da sustentação em perfil RAE $2822 \operatorname{com} \theta=3^{\circ} \ldots 82$

4.38 Sensibilidade da sustentação em perfil RAE $2822 \operatorname{com} \theta=4^{\circ} \ldots 82$

4.39 Malhas RAE $2822 \ldots \ldots \ldots$. . . . . . . . . . . . 83 
4.40 Comparação da gradiente $\partial L / \partial p_{o}$ em diferentes malhas do perfil RAE $2822 \operatorname{com} \theta=3^{\circ} \ldots \ldots \ldots \ldots$

4.41 Contornos de número de Mach do perfil RAE $2822\left(M_{\infty}=0.8\right.$, $\left.\theta=3^{\circ}\right) \ldots \ldots \ldots \ldots \ldots \ldots$

4.42 Histórico de convergência do perfil RAE $2822\left(M_{\infty}=0.8, \theta=3^{\circ}\right) \quad 85$

4.43 Variação de entropia, $\Delta s$, em perfil RAE $2822\left(M_{\infty}=0.8, \theta=3^{\circ}\right) \quad 86$

4.44 Contornos de número de Mach do perfil RAE $2822\left(M_{\infty}=0.9\right.$, $\left.\theta=3^{\circ}\right) \ldots \ldots \ldots \ldots \ldots \ldots \ldots$

4.45 Sensibilidade do momento em perfil RAE $2822\left(\theta=0^{\circ}\right) \ldots . . .88$

4.46 Sensibilidade do momento em perfil RAE $2822\left(\theta=1^{\circ}\right) \ldots$. . . 88

4.47 Sensibilidade do momento em perfil RAE $2822\left(\theta=2^{\circ}\right) \ldots . . \quad 88$

4.48 Sensibilidade do momento em perfil RAE $2822\left(\theta=3^{\circ}\right) \ldots . . .89$

4.49 Sensibilidade do momento em perfil RAE $2822\left(\theta=4^{\circ}\right) \ldots . \quad$. . 89

4.50 Solução do perfil NACA $0012\left(M_{\infty}=0.8, \alpha=2^{\circ}\right) \ldots$. . . . . 90

4.51 Solução adjunta do perfil NACA $0012\left(M_{\infty}=0.8, \alpha=2^{\circ}\right) \quad \ldots \quad .90$

4.52 Sensibilidade da sustentação em perfil NACA $0012\left(\theta=0^{\circ}\right)$. . . 91

4.53 Sensibilidade da sustentação em perfil NACA $0012\left(\theta=1^{\circ}\right)$. . . 91

4.54 Sensibilidade da sustentação em perfil NACA $0012\left(\theta=2^{\circ}\right)$. . . 91

4.55 Sensibilidade da sustentação em perfil NACA $0012\left(\theta=3^{\circ}\right) \ldots . .92$

4.56 Sensibilidade da sustentação em perfil NACA $0012\left(\theta=4^{\circ}\right)$. . . . 92

4.57 Malhas NACA0012 . . . . . . . . . . . . . . . . 93

4.58 Comparação da sensibilidade $\partial L / \partial \theta$ em diferentes malhas do perfil NACA $0012 \operatorname{com} \theta=2^{\circ} \ldots \ldots \ldots \ldots$. . . . . . . . . 94

4.59 Contornos do número de Mach do perfil NACA $0012\left(M_{\infty}=0.8\right.$, $\left.\theta=2^{\circ}\right) \ldots \ldots \ldots \ldots \ldots$

4.60 Histórico de convergência das soluções físicas do perfil NACA 0012 $\left(M_{\infty}=0.8, \theta=2^{\circ}\right) \ldots \ldots \ldots \ldots \ldots$

4.61 Contornos de número de Mach do perfil NACA $0012\left(M_{\infty}=0.8\right.$, $\theta=2^{\circ} \ldots \ldots \ldots \ldots \ldots \ldots \ldots$ 
4.62 Contornos de número de Mach do perfil NACA $0012\left(M_{\infty}=0.9\right.$, $\theta=2^{\circ} \ldots \ldots \ldots \ldots \ldots \ldots \ldots$

4.63 Sensibilidade do momento em perfil NACA $0012\left(\theta=0^{\circ}\right) \ldots$

4.64 Sensibilidade do momento em perfil NACA $0012\left(\theta=1^{\circ}\right) \ldots$. . 97

4.65 Sensibilidade do momento em perfil NACA $0012\left(\theta=2^{\circ}\right) \ldots . . .97$

4.66 Sensibilidade do momento em perfil NACA $0012\left(\theta=3^{\circ}\right) \ldots . . .97$

4.67 Sensibilidade do momento em perfil NACA $0012\left(\theta=4^{\circ}\right) \ldots$

C.1 Diagrama $\theta-\beta-M \ldots \ldots \ldots \ldots$ 


\section{LISTA DE TABELAS}

3.1 Resumo das condições de contorno adjuntas de saída . . . . . . . 50

3.2 Resumo das condições de contorno adjuntas de entrada . . . . . . 59

4.1 Condições de contorno impostas em bocal divergente subsônico . . 67

4.2 Condições de contorno impostas em escoamentos subsônicos/transônicos externos . . . . . . . . . . . . . . . . . . . . 79

4.3 Malhas RAE $2822 \ldots \ldots$. . . . . . . . . . . . . . . . 83

4.4 Malhas NACA0012 . . . . . . . . . . . . . . . . . 93

A.1 Coeficientes de Riemann adjuntos . . . . . . . . . . . . . 110 


\section{ACRÔNIMOS}

CFD Computational Fluid Dynamics

CNPq Conselho Nacional de Desenvolvimento Científico e Tecnológico CST Class Shape-function Transformation

EPUSP Escola Politécnica da Universidade de São Paulo

ESSS Engineering Simulation and Scientific Software

FAPESP Fundação de Amparo à Pesquisa do Estado de São Paulo

MGM Modified Garabedian-McFadden

NACA National Advisory Committee for Aeronautics

NDF Núcleo de Dinâmica dos Fluidos

PDE Partial Differential Equation

RAE Royal Aircraft Establishment

RANS Reynolds-Averaged Navier-Stokes 


\section{NOMENCLATURA}

a Vetor de variáveis adjuntas que garantem que parâmetros de controle assumam valores referentes a uma configuração base

A Matriz Jacobiana de fluxo das variáveis conservadas na dir. coordenada $x$

A Matriz Jacobiana das variáveis primitivas na direção coordenada $x$

$\mathbb{A}_{K} \quad$ Matriz Jacobiana associada à uma direção $\vec{k}$

$\mathcal{B} \quad$ Condições de contorno do problema físico

$\mathcal{B}^{*} \quad$ Condições de contorno do problema adjunto

$b_{c p} \quad$ Planos de corte

$b_{\infty} \quad$ Farfield

B Matriz Jacobiana de fluxo das variáveis conservadas na dir. coordenada $y$

B Matriz Jacobiana das variáveis primitivas na direção coordenada $y$

$b_{w} \quad$ Superfície sólida

c Velocidade do som

$C_{p} \quad$ Calor específico à pressão constante

$C_{v} \quad$ Calor específico à volume constante

$\mathcal{D} \quad$ Domínio

E Vetor de fluxos na direção $x$

e $\quad$ Energia específica

$e_{i} \quad$ Energia interna específica

F Vetor de fluxos na direção $y$

$\vec{f}_{e} \quad$ Vetor de forças externas

$f_{\alpha}^{k^{\prime}} \quad$ Vetor de fluxo em coordenadas cartesianas associado a direção $x^{k^{\prime}}$ 
$\mathcal{F} \quad$ Funcional objetivo genérico

G Funcional aumentado

$\mathcal{G} \quad$ Geometria de uma superfície aerodinâmica genérica

$g^{i^{\prime} k^{\prime}} \quad$ Tensor métrico

$H \quad$ Entalpia total

I $\quad$ Medida de mérito

$\hat{\imath} \quad$ Versor da coordenada cartesiana $x$

$J \quad$ Jacobiano da transformação de coordenadas

$\hat{\jmath} \quad$ Versor da coordenada cartesiana $y$

$\vec{k} \quad$ Vetor genérico

$\hat{k} \quad$ Versor da coordenada cartesiana $z$

$k_{x} \quad$ Componente cartesiana do vetor $\vec{k}$ na direção $x$

$k_{y} \quad$ Componente cartesiana do vetor $\vec{k}$ na direção $y$

L Matriz de transformação de similaridade que diagonaliza de $\tilde{A}_{n}$

$\mathcal{L} \quad$ Forma linearizada das equações não-lineares $\mathbf{N}$

$\mathcal{L}^{*} \quad$ Operador adjunto de $\mathcal{L}$

$\mathcal{M}$ Matriz resultante da decomposição do concomintante bilinear

M Matriz de transformação das variáveis conservadas para primitivas

N Sistema de equações diferenciais não-lineares que governam o escoamento

P Matriz de transformação de similaridade que diagonaliza de $A_{n}$

$p \quad$ Pressão estática

$P_{1} \quad$ Operador resultante da decomposição do concomintante bilinear

$\mathcal{P} \quad$ Parâmetros de controle de um sistema

$P[]_{s}$ Concomitante bilinear

$p_{d} \quad$ Pressão desejada 
$\vec{q} \quad$ Vetor de fluxo de calor

Q Vetor de variáveis de estado

$R \quad$ Constante do gás

R Termo fonte das equações que governam o escoamento

S Linearização das equações governantes que agrupa variações dos parâmetros de controle

$\mathcal{S} \quad$ Área da superfície do volume $\mathcal{V}$

T Temperatura absoluta

$u \quad$ Componente $x$ do vetor velocidade

$U_{n} \quad$ Velocidade do fluido perpendicular a uma fronteira

$v \quad$ Componente $y$ do vetor velocidade

$\overrightarrow{\mathbf{v}} \quad$ Vetor velocidade

$\mathcal{V} \quad$ Volume do domínio computacional

V Vetor de variáveis primitivas

W Vetor de variáveis características

$x \quad$ Coordenada cartesiana horizontal

y Coordenada cartesiana vertical

Subscritos

$s \quad$ Implica condições impostas em fronteiras apropriadas

\section{Símbolos Gregos}

$\beta_{i^{\prime}}^{k} \quad$ Operador transformação de coordenadas

$\chi \quad$ Coordenadas no espaço de fase

$\partial \mathcal{D} \quad$ Fronteiras do domínio

$\eta \quad$ Coordenada generalizada normal

$\gamma \quad$ Razão de calores específicos 

$\Lambda \quad$ Matriz diagonal
$\lambda \quad$ Passo do processo de otimização
$\nabla \quad$ Operador divergente
$\Phi \quad$ Vetor de variáveis adjuntas $\phi_{\alpha}$ no domínio
$\Psi \quad$ Vetor de variáveis adjuntas $\psi_{\alpha}$ nas fronteiras do domínio
$\rho \quad$ Massa específica
$\sigma(\vec{k}) \quad$ Autovalor da matriz $\mathbb{A}_{K}$
$\tau \quad$ Coordenada generalizada temporal
$\xi \quad$ Coordenada generalizada tangencial 


\section{INTRODUÇÃO}

\subsection{O Projeto Aerodinâmico}

Apesar de depender da missão a qual se destina, o projeto de aeronaves visa, de uma maneira geral, otimizar a forma geométrica de uma configuração, levando em conta uma relação de compromisso entre desempenho aerodinâmico, peso estrutural e especificações de volume interno para armazenamento de combustível e carga útil (REUTHER; JAMESON, 1994). O procedimento adotado para que se obtenha tal configuração ótima, no entanto, evoluiu consideravelmente ao longo dos anos. Devido à complexidade do escoamento de fluidos, conhecidamente regido por equações diferenciais a derivadas parciais (PDE's) não-lineares, a estratégia utilizada no projeto aerodinâmico em tempos mais remotos era baseada em métodos empíricos em que, a partir da análise do desempenho de uma geometria base através de ensaios realizados em túneis de vento, eram propostas alterações até que se obtivesse uma forma que estivesse de acordo com os requisitos impostos.

O enorme progresso experimentado pela Dinâmica dos Fluidos Computacional (CFD) nas últimas décadas, por sua vez, ensejou uma revolução no modo de se projetar superfícies aerodinâmicas. O desenvolvimento de métodos mais robustos e confiáveis aliado a crescente disponibilidade de recursos computacionais, tanto no que diz respeito à capacidade de armazenamento quanto à velocidade de processamento, alçaram o que era inicialmente apenas um instrumento de estudo para problemas físicos em nível de investigação científica à posição de poderosa ferramenta utilizada para se obter soluções dos mais complexos problemas de engenharia (MALISKA, 2004). Além de, geralmente, permitir a avaliação de diferentes configurações a custos relativamente mais baixos do que ensaios em túneis de vento, a CFD ainda possibilitou a simulação do movimento de fluidos em regimes mais difíceis de se reproduzir experimentalmente como, por exemplo, escoamentos hipersônicos a grandes altitudes.

Apesar de reduzir custos na indústria, a CFD era utilizada apenas como ferramenta de análise e o método de tentativa e erro continuava a ser aplicado durante 
a fase de projeto. Entretanto, é improvável que este processo iterativo resulte em um produto verdadeiramente ótimo. Além disso, o natural aumento da complexidade dos projetos e o consequente aumento do número de parâmetros envolvidos no processo dificultavam as decisões de seus responsáveis. Isso ocorria porque não havia como garantir que esse procedimento explorasse adequadamente o espaço de soluções viáveis. Foi então que, com o desenvolvimento de métodos de otimização e projeto inverso, ocorreu uma segunda revolução no projeto aerodinâmico. Combinados com recursos de simulação de escoamentos, esses métodos acrescentaram uma nova dimensão à CFD, ao aliarem a já conhecida capacidade de análise à possibilidade de investigar de forma eficiente o espaço de projeto.

\subsection{Métodos de Otimização e Projeto Inverso}

Em geral, o projetista tem uma idéia do tipo de distribuição de pressão sobre a superfície aerodinâmica que resultaria no desempenho desejado. Assim, seria interessante se houvesse a possibilidade de determinar a geometria que corresponde a uma dada distribuição de pressão objetivo sob certas condições prescritas. Dessa forma conseguir-se-ia, por exemplo, evitar gradientes de pressão adversos que induziriam à separação prematura da camada limite em aerofólios. Conhecido como projeto inverso aerodinâmico, esse problema foi estudado pela primeira vez por Lighthill (1945). Seu trabalho consistia em projetar perfis de asas bidimensionais em escoamentos representados pelo modelo potencial incompressível, utilizando a técnica de transformação conforme de um aerofólio para o círculo unitário. Esse tipo de mapeamento, no entanto, não permitia a aplicação do método em escoamentos compressíveis, já que a presença de ondas de choque na solução provocaria uma descontinuidade na função de transformação. McFadden (1979) contornou esse problema através da adição de dissipação artificial para suprimir a presença de choques em escoamentos transônicos.

Apesar das vantagens descritas, essa classe de métodos não garante que a distribuição desejada, que deve ser especificada a priori, seja realizável ${ }^{1}$, ou que implique em melhores coeficientes aerodinâmicos. Além disso, não se pode garantir que a configuração obtida represente uma solução ótima para as condições prescritas (REUTHER; JAMESON, 1994). Os métodos de otimização, por outro lado, proporcionam uma abordagem mais abrangente do projeto aerodinâmico. Essencialmente, eles buscam extremos de funções de mérito previamente defini-

\footnotetext{
${ }^{1}$ Entenda-se por realizável uma distribuição de pressão que satisfaça as equações que governam o escoamento.
} 
das, que representam pontos de máximo ou mínimo locais no espaço de projeto. De qualquer modo, a existência de um extremo, ainda que local, deve corresponder a uma configuração ótima. Para diferenciar esses métodos da tradicional forma de projeto por tentativa e erro, eles procuram avaliar a sensibilidade de cada medida de mérito com respeito a variações dos parâmetros através de suas derivadas. Entretanto, esses funcionais geralmente envolvem variáveis físicas do escoamento que, por sua vez, também dependem dos parâmetros de projeto. Infelizmente, essa dependência só é conhecida de forma fechada em um número muito pequeno de casos simples, que não cobrem o universo de aplicações de interesse (VOLPE, 2011). Por outro lado, quando se consegue estimar esses gradientes de sensibilidade - e há meios de o fazer - então se pode fazer uso de poderosos métodos de busca baseados neles (VANDERPLAATS, 1984).

Contudo, nem todos os métodos de otimização são baseados em gradiente. Dentre os que não fazem uso dessa informação para realizar o processo de otimização, destacam-se os chamados algoritmos genéticos (GOLDBERG, 1989), baseados no processo biológico de seleção natural. Neles, a partir de uma população inicial de soluções base, são selecionadas aquelas mais bem adaptadas, ou seja, as que apresentam melhores valores da medida de mérito em questão, para então promovê-las com maior probabilidade de serem utilizadas em uma recombinação para gerar novas soluções. Assim como ocorre na natureza, por vezes, impõem-se a ocorrência de mutações em seus produtos. As novas soluções obtidas substituem, então, algumas ou todas as soluções da população antiga. Esta classe de métodos é, particularmente, útil em casos em que a função objetivo não é contínua e, portanto, não é diferenciável, quando há um conjunto de medidas de mérito independentes a serem consideradas, ou então, quando se espera que existam múltiplos pontos de ótimo local. Zingg, Nemec e Pulliam (2008) comparam a evolução dos algoritmos genéticos com um método baseado em gradiente em aplicações de otimização aerodinâmica e concluem que os primeiros são mais apropriados para o projeto preliminar, onde modelos mais simplificados são tipicamente utilizados, enquanto algoritmos baseados em gradiente são mais adequados em uma fase posterior, em que o projeto é mais detalhado e, consequentemente, existe a necessidade de simulações mais fiéis a física.

A utilização de técnicas numéricas para otimização de perfis aerodinâmicos em escoamentos transônicos foi iniciada por Hicks, Murman e Vanderplaats (1974) e, posteriormente, estendida para o projeto de asas por Hicks e Henne (1978). Os últimos exploraram a possibilidade de combinar objetivos de projeto desejados com o processo de otimização com restrições. Nesse caso, a configuração é 
especificada por um conjunto de parâmetros. O método de otimização, então, seleciona valores desses parâmetros que maximizam alguma medida de mérito como a razão da sustentação pelo arrasto, sujeita a restrições como espessura e volume da asa. Em princípio esse método permite ao projetista especificar quaisquer objetivos de projeto razoáveis. Entretanto, o método se torna extremamente caro a medida em que o número de parâmetros aumenta e, na prática, o sucesso das suas aplicações depende fortemente da escolha da representação paramétrica da configuração.

Desde então, diversos métodos de otimização aerodinâmica foram desenvolvidos, dentre eles o método Modified Garabedian-McFadden (MGM) baseado no modelo potencial linearizado do escoamento (SANTOS, 1993; VOLPE, 2004; VOLPE, 2005; VOLPE et al., 2007) e o método adjunto (JAMESON, 1988; REUTHER, 1996; SANTOS, 1995; JAMESON; NADARAJAH, 2000; JAMESON; SRIRAM; MARTINELLI, 2003; JAMESON; KIM, 2003a), que ganhou destaque por permitir grande flexibilidade quanto ao modelo adotado para a física do escoamento e para a medida de mérito. Por isso serve tanto para problemas de otimização como de projeto inverso. Também tem o atrativo de tornar o custo computacional aproximadamente independente do número de parâmetros de controle, como será visto mais adiante. Tais características fizeram desse método um prolífico tema de pesquisa.

\subsection{O Método Adjunto e Suas Aplicações}

O método adjunto, baseado na teoria de controle de sistemas governados por equações diferenciais (LIONS, 1971), foi inicialmente proposto por Pironneau (1983) para otimização de forma em problemas elípticos e, mais tarde, estendido para escoamentos transônicos por Jameson (1988), que utilizou seu conhecimento sobre teoria de controle ótimo para desenvolver o que ele próprio chama de métodos de projeto ótimo (GILES; PIERCE, 2000b).

A continuidade dos trabalhos de Jameson e seus colaboradores resultou no desenvolvimento das formulações adjuntas para escoamentos modelados pela equação potencial (JAMESON, 1988), equações de Euler (REUTHER, 1996) e NavierStokes (JAMESON; MARTINELLI; PIERCE, 1997). Além disso, a complexidade das aplicações de seu trabalho também progrediu desde a otimização de aerofólios 2-D (JAMESON, 1990) utilizando a técnica de transformação conforme, passando por projeto de asas 3-D (JAMESON, 1994), estudos de interferência aerodinâmica com interação asa isolada/asa-fuselagem (REUTHER; JAMESON, 1995) e, final- 
mente, chegando a configurações completas de aeronaves (REUTHER et al., 1996).

Posteriormente, Kim (2001) ampliou as investigações a respeito do método com estudos voltados para a otimização de aerofólios em configurações que utilizam mecanismos hipersustentadores como flaps e slats em escoamentos modelados pelas equações de Navier-Stokes com média de Reynolds (RANS). Em seguida, Alonso e Kroo (2002) fizeram uso de algoritmos de otimização para redução de ruído em plataformas supersônicas e, pouco depois, Jameson, Sriram e Martinelli (2003) conseguiram aplicar o método em malhas não-estruturadas, fato que certamente ampliou sua utilidade, na medida em que possibilitou a otimização de geometrias mais complexas. Aplicações ainda foram desenvolvidas para escoamentos não-estacionários (THOMAS; HALL; DOWELL, 2005; NADARAJAH; JAMESON, 2006; NADARAJAH; JAMESON, 2007; MANI; MAVRIPLIS, 2008; ECONOMON; PALACIOS; ALONSO, 2014), escoamentos hipersônicos (KLINE et al., 2015; KLINE; ECONOMON; ALONSO, 2016), além de trabalhos propostos nas áreas de interferência aerodinâmica e integração propulsiva (FUJITA, 2002; MAKINO; IWAMIYA; LEI, 2003; RODRIGUEZ, 2003; KIM; KOC; NAKAHASHI, 2005).

As aplicações do método adjunto, no entanto não se resumem à aerodinâmica externa. Duta, Giles e Campobasso (2002) aplicaram o método em escoamentos internos ao otimizar turbo-máquinas. Já Giles e Pierce (1997) investiam em estudos mais teóricos do método como condições de contorno, comportamento da solução, propriedades das equações (GILES; PIERCE, 1998a), e até mesmo solução analítica para o caso de escoamentos governados pelas equações de Euler quase unidimensionais (GILES; PIERCE, 2000a; GILES; PIERCE, 2001).

Também se desenvolveram aplicações do método adjunto para aumentar a precisão de funcionais relevantes, como medidas de sustentação e arrasto (GILES; PIERCE, 1998b; GILES; PIERCE, 1999a; GILES; PIERCE, 1999b; PIERCE; GILES, 2004). Uma abordagem semelhante a essa deu origem a uma nova classe de aplicações, que se voltaram à análise de erros em CFD (GILES; PIERCE; SÜLI, 2004; GILES; SÜLI, 2002). Seguindo essa linha, Venditti e Darmofal (1999) iniciaram suas pesquisas sobre o método adjunto para estimativa de erros e adaptação de malhas. Seus estudos comprendem desde escoamentos quase-1D (VENDITTI; DARMOFAL, 2000), passando por escoamentos 2-D invíscidos (VENDITTI; DARMOFAL, 2002) e adaptação anisotrópica em escoamentos viscosos (VENDITTI; DARMOFAL, 2003). Mais tarde, Nielsen et al. (2004) trabalharam com escoamentos turbulentos em malhas não-estruturadas e Martins, Alonso e Reuther (2005), por sua vez, contribuiram para a evolução do estado da arte na área de otimização ao utilizar o método adjunto para integrar duas áreas do projeto de aeronaves: aerodinâmica 
e estruturas. Alguns anos depois, Palaniappan et al. (2011) conseguiram utilizar o método adjunto para controlar um fenômeno físico associado a estabilidade de sistemas aeroelásticos conhecido como flutter.

Vale destacar que a solução do problema adjunto envolve aproximadamente o mesmo custo computacional que a simulação do escoamento propriamente dita. Ainda assim há trabalhos que procuram reduzir mais esses custos. Nesse sentido, Taasan, Kuruvila e Salas (1992) implementaram o método one shot em que as equações que governam o escoamento, usualmente impostas como restrições do problema variacional, são requeridas para satisfazer apenas a solução final convergida (KURUVILA; TAASAN; SALAS, 1994). Em seu trabalho, os custos computacionais também foram reduzidos ao aplicar técnicas multigrid às modificações da geometria, bem como à solução das equações do escoamento e adjuntas. Seu trabalho foi explorado por Beux e Dervieux (1993) ao considerar a influência da forma de parametrização da geometria no desempenho do método adjunto (MARCO; BEUX, 1993). As aplicações do método adjunto, entretanto, não param por aí. Sua versatilidade possibilitou aplicações nas mais diferentes áreas da engenharia, envolvendo temas que vão da termo-hidráulica de reatores nucleares às ciências atmosféricas (CACUCI et al., 1980; HALL; CACUCI, 1983).

A eficiência deste método, naturalmente, instigou o interesse da indústria a utilizá-lo na fase de concepção de aeronaves como, por exemplo, no projeto aerodinâmico do McDonnel Douglas MDXX (JAMESON, 1997), onde, ainda, predominam métodos de projeto inverso associados a modelos específicos do escoamento como o potencial compressível linearizado (NAIK et al., 1995; OLIVEIRA; TRAPP; MACEDO, 2003; SADRI; LEBLOND; PIPERNI, 2002).

\subsection{Formulação do Problema Adjunto}

No contexto da teoria de controle de Lions (1971), uma superfície aerodinâmica pode ser entendida como um dispositivo que controla o escoamento. Ao impor as equações da mecânica dos fluidos como restrições do problema variacional, as variações da medida de mérito são projetadas no espaço de soluções realizáveis, o que reduz sensivelmente o custo computacional para obtenção de sensibilidades com relação ao escoamento de interesse. Matematicamente, o problema consiste em minimizar uma função de mérito sob certas restrições, que são representadas por um conjunto de PDE's chamadas de equações de estado. 


\subsubsection{Formas de Discretização da Equação Adjunta}

A implementação numérica do método adjunto requer a utilização de uma forma discretizada da equação adjunta. Existem, basicamente, duas formas possíveis para sua obtenção. Primeiro, ela pode ser derivada à partir da forma discretizada das equações que governam o escoamento e, portanto, seria obtida diretamente em uma forma discretizada. Esta forma, conhecida na literatura como formulação discreta do método, fica, no entanto, condicionada à forma como as equações da mecânica dos fluidos foram discretizadas, tanto no que diz respeito à complexidade, quanto à precisão dos resultados. Este procedimento ainda pode levar a expressões bastante complexas, especialmente quando se usam aproximações de ordem superior para as equações governantes.

Alternativamente, a equação adjunta pode ser derivada a partir da forma original das equações que governam o escoamento para, só então, ser discretizada. Esta é a formulação contínua do método. A equação adjunta pode ser, então, resolvida de modo independente dos métodos utilizados para obter a solução das equações do escoamento. Deve-se ressaltar que a forma discretizada da equação adjunta obtida na formulação contínua não coincide com aquela da formulação discreta. Pode-se atribuir esta diferença ao fato de que, nesta última, a restrição que se está impondo corresponde a uma particular discretização das equações da mecânica dos fluidos, ao invés das equações originais.

Em princípio, se a discretização das equações da mecânica dos fluidos é consistente, de modo que a solução da equação modificada (forma discreta) se aproxime da solução exata do problema original à medida em que o tamanho do passo de tempo e dos elementos da malha computacional tendam a zero $(\Delta t \rightarrow 0$, $\Delta x \rightarrow 0)$ de forma independente, então os resultados da formulação discreta do método adjunto também devem se aproximar àqueles da formulação contínua com o refinamento da malha, uma vez que eles impõe precisamente essa aproximação consistente do escoamento.

Jameson e Nadarajah (2000) comparam ambas as formulações do método adjunto e concluem que, no procedimento utilizado por eles, os gradientes adjuntos discretos são mais condizentes com os gradientes obtidos por diferenças finitas do que os gradientes contínuos, mas as diferenças são geralmente pequenas. Entretanto, a convergência da função de mérito não é afetada significativamente quando se utiliza o gradiente discreto ao invés do contínuo. Consequentemente, não se encontra um benefício particular em utilizar o método adjunto discreto. 


\subsubsection{Abordagem Tradicional — Jameson}

Medidas de mérito relevantes às aplicações aerodinâmicas podem representar uma medida de erro quadrática de uma dada distribuição de pressão $\left(c_{p}\right)$ com relação a uma distribuição objetivo $\left(c_{p t}\right)$ na superfície de um corpo sólido $\left(b_{w}\right)$, a energia cinética do campo de perturbações no domínio $(\mathcal{D})$ como um todo, ou ainda a média temporal da força que o fluido exerce sobre uma superfície sólida $(\mathbf{n} \cdot \boldsymbol{\sigma})_{b_{w}}$, projetada em uma dada direção $(\mathbf{e})$, como mostram as expressões 1.1 abaixo:

$$
I=\oint_{b_{w}} \frac{\left(c_{p}-c_{p t}\right)^{2}}{2} \mathrm{~d} S ; K=\int_{\mathcal{D}} \frac{\rho u^{2}}{2} \mathrm{~d} V ; \overline{C_{f}}=\frac{1}{T} \int \oint_{0 b_{w}}^{T} \frac{\mathbf{n} \cdot \boldsymbol{\sigma} \cdot \mathbf{e}}{\frac{\rho_{\infty} U_{\infty}^{2}}{2}} \mathrm{~d} S \mathrm{~d} t
$$

Geralmente, essas medidas de mérito envolvem, portanto, funcionais ou funções que dependem das variáveis do escoamento, $\mathbf{Q}$, e da geometria, $\mathcal{G}$, de suas fronteiras (JAMESON; KIM, 2003b; REUTHER, 1996). Assim, podem ser representadas de maneira geral segundo a expressão:

$$
I[\mathbf{Q}, \mathcal{P}]=\int_{\chi} \mathcal{F}[\mathbf{Q}(\chi), \mathcal{P}(\chi), \chi] \mathrm{d} \chi
$$

onde o vetor $\mathbf{Q}$ representa as coordenadas do sistema analisado, no espaço de estado. O vetor $\chi$ representa as coordenadas dos pontos no espaço de fase nas aplicações aqui consideradas, $\chi$ corresponde ao espaço físico. Finalmente, o vetor $\mathcal{P}$ representa, da forma mais geral, os parâmetros que controlam o sistema, a exemplo daqueles que especificam a geometria de um corpo.

$$
\begin{aligned}
\mathbf{Q}(\chi) & =\left[Q_{1}(\chi), \ldots Q_{K}(\chi)\right] \\
\chi & =\left(\chi_{1}, \ldots, \chi_{J}\right) \\
\mathcal{P}(\chi) & =\left[\mathcal{P}_{1}(\chi), \ldots, \mathcal{P}_{L}(\chi)\right]
\end{aligned}
$$

Afim de facilitar a notação aqui utilizada, considere, as seguintes definições de produto interno no espaço de fase:

$$
\langle\mathbf{f}, \mathbf{g}\rangle \equiv \int_{\mathcal{D}} \mathbf{f}(\chi) \cdot \mathbf{g}(\chi) \mathrm{d} \mathcal{V} \quad ; \quad\langle\mathbf{f}, \mathbf{g}\rangle_{s} \equiv \int_{\partial \mathcal{D}} \mathbf{f}(\chi) \cdot \mathbf{g}(\chi) \mathrm{d} \mathcal{S}
$$

no domínio e em suas fronteiras, respectivamente.

Variações da geometria podem ser produzidas por alterações nos parâmetros $\delta \mathcal{P}$ e, naturalmente, elas implicam em variações no campo do escoamento $\delta \mathbf{Q}$. Assim, em princípio, a sensibilidade da medida de mérito a variações da geometria 
é medida pela primeira variação de Gâteaux (LUSTERNICK; SOBOLEV, 1961) do funcional $I$, dada pela expressão:

$$
\delta I=\underbrace{\left\langle\mathcal{F}_{\mathbf{Q}}^{\prime}, \delta \mathbf{Q}\right\rangle}_{\delta I_{\mathbf{Q}}}+\underbrace{\left\langle\mathcal{F}_{\mathcal{P}}^{\prime}, \delta \mathcal{P}\right\rangle}_{\delta I_{\mathcal{P}}}
$$

onde o primeiro termo da eq. (1.7), $\delta I_{\mathbf{Q}}$, corresponde a parte física $\delta \mathbf{Q}(\chi)$ da variação total $\delta I$, enquanto o segundo, $\delta I_{\mathcal{P}}$, está relacionado a parte paramétrica $\delta \mathcal{P}(\chi)$. Uma vez que a geometria da superfície aerodinâmica é descrita por parâmetros conhecidos, o termo $\mathcal{F}_{\mathcal{P}}^{\prime}$ pode ser, geralmente, determinado de maneira analítica e, consequentemente, a variação $\delta I_{\mathcal{P}}$ é facilmente obtida. O que torna a avaliação da variação total difícil é parte física da sua variação. Embora $\mathcal{F}_{\mathbf{Q}}^{\prime}$ possa ser determinado, o termo $\delta \mathbf{Q}(\chi)$ é raramente conhecido, uma vez que, em geral, não se conhece explicitamente a dependência das variáveis do escoamento com respeito aos parâmetros que controlam a geometria devido à complexidade das equações diferenciais não-lineares que governam os escoamentos. Além disso, não é possível assegurar a realizabilidade das variações no campo do escoamento $\delta \mathbf{Q}$, que não foi imposta à eq. (1.7). Neste ponto, vale ressaltar que as variações $\delta \mathbf{Q}$ são deslocamentos virtuais e, portanto, representam soluções possíveis. Este conceito é de fundamental importância para o entendimento do método adjunto e será destacado outras vezes ao longo desta tese.

Não sendo possível determinar a sensibilidade de $I$ através da eq. (1.7), a forma mais direta de se atacar o problema seria avaliar o gradiente $\partial I / \partial \mathcal{P}_{\alpha}$ por diferenças finitas. Em linhas gerais, o procedimento envolveria obter a solução convergida para a geometria original $\mathcal{G}(\chi, \mathcal{P})$, que seria a solução base. Em seguida, cada um dos parâmetros de $\mathcal{G}$ seria perturbado individualmente, ou seja, $\mathcal{P}_{\alpha}+\delta \mathcal{P}_{\alpha}$, enquanto os demais manteriam seus valores originais. Para cada uma dessas perturbações, seria obtida a solução convergida correspondente. Os valores da medida de mérito seriam calculados para as soluções perturbadas e a solução base. De posse desses resultados, seria possível estimar numericamente, em primeira ordem, a sensibilidade de $I$ com relação a cada um dos parâmetros que definem a geometria através da seguinte expressão:

$$
\frac{\partial I}{\partial \mathcal{P}_{\alpha}} \cong \frac{I\left(\mathcal{P}_{\alpha}+\delta \mathcal{P}_{\alpha}\right)-I\left(\mathcal{P}_{\alpha}\right)}{\delta \mathcal{P}_{\alpha}}
$$

Então, o vetor gradiente $\partial I / \partial \mathcal{P}_{\alpha}$, que tem sua precisão limitada pela magnitude da variação de cada um dos parâmetros $\delta \mathcal{P}_{\alpha}$, pode ser utilizado para determinar a direção de otimização. Para tanto, é possível utilizar, por exemplo, o método steepest descent (PIRONNEAU, 1983) que aplica um passo fixo $\lambda$ 
no sentido negativo do gradiente, obtendo os novos parâmetros otimizados $\mathcal{P}_{\alpha}$ da seguinte maneira:

$$
\mathcal{P}_{\alpha}^{n+1}=\mathcal{P}_{\alpha}^{n}-\lambda \frac{\partial I}{\partial \mathcal{P}_{\alpha}}
$$

Este procedimento assegura a realizabilidade das variações $\delta \mathbf{Q}$ à medida que busca uma solução convergida do escoamento para cada uma das perturbações. Entretanto, ele o faz a custa de grande aumento do esforço computacional. Justamente porque requer uma solução convergida para cada perturbação $\delta \mathcal{P}_{\alpha}$. Em resumo, se $\mathcal{G}$ tem $N$ parâmetros, então são necessárias $N+1$ soluções independentes das equações do movimento, levando-se em conta a solução base (JAMESON; NADARAJAH, 2000), para conseguir obter o gradiente da medida de mérito com relação aos parâmetros que descrevem a geometria da superfície aerodinâmica que se quer otimizar. É evidente que o custo computacional desta abordagem se torna proibitivo à medida que aumenta o número de parâmetros que controlam $\mathcal{G}(\chi, \mathcal{P})$.

À princípio, uma forma de contornar esta dificuldade seria restringir, a priori, as variações das grandezas físicas $\delta \mathbf{Q}$ ao espaço das soluções realizáveis, então, talvez, fosse possível eliminar a necessidade dos caros cálculos de soluções perturbadas para estimar o gradiente de sensibilidade. O método adjunto explora esta possibilidade por meio de conceitos de teoria de controle para alcançar este objetivo. Basicamente, ele impõe as equações que governam o escoamento como restrições ao problema variacional e, com isso, impede soluções não realizáveis. Para tanto, considera-se que a solução dessas equações depende das variáveis do escoamento e da forma de suas fronteiras. Em termos gerais, supondo que as equações que governam o escoamento componham um sistema de $K$ equações diferenciais não-lineares $\mathbf{N}$, submetidas a um conjunto de condições de contorno correspondentes $\mathcal{B}$. Em termos de operadores, teria-se:

$$
\begin{aligned}
\mathbf{N}[\mathbf{Q}(\chi), \mathcal{P}] & =\mathbf{R}(\chi, \mathcal{P}) \\
\mathcal{B}[\mathbf{Q}(\chi)]_{s} & =0
\end{aligned}
$$

onde o subscrito [ ] implica que as condições são impostas em fronteiras apropriadas.

Então, poderia-se em princípio, construir um funcional aumentado $G$ que representa o problema variacional submetido a restrições, geralmente não-holo- 
nômicas ${ }^{2}$, na forma:

$$
G(\mathbf{Q}, \mathcal{P}, \Phi)=I[\mathbf{Q}, \mathcal{P}]-\langle\Phi, \mathbf{N}-\mathbf{R}\rangle-\langle\Psi, \mathcal{B}\rangle_{s}
$$

onde os vetores $\Phi$ e $\Psi$ correspondem aos multiplicadores de Lagrange que impõem as equações que governam o escoamento e suas condições de contorno, respectivamente, e que podem ser vistos, portanto, como condições de realizabilidade.

A determinação dos extremos de $G$ requer o cálculo de sua variação, $\delta G$. Para isso, consideram-se as equações que governam a física, (1.10). Suas diferenciais de Fréchet são dadas por:

$$
\begin{aligned}
\mathcal{L} \delta \mathbf{Q} & =\mathbf{S} \delta \mathcal{P} \\
\mathcal{B}_{\mathbf{Q}}^{\prime} \delta \mathbf{Q} & =0
\end{aligned}
$$

onde se definem os operadores $\mathcal{L} \equiv \mathbf{N}_{\mathbf{Q}}^{\prime}$ e $\mathbf{S} \equiv \mathbf{R}_{\mathcal{P}}^{\prime}-\mathbf{N}_{\mathcal{P}}^{\prime}$. O primeiro deles, $\mathcal{L}$, representa a forma linearizada das equações originais, enquanto o segundo agrupa as variações dos parâmetros de controle.

Para se calcular a primeira variação de (1.11), observa-se que o primeiro componente de seu lado direito já tem sua variação determinada em (1.7). Avaliando a variação de Gâteaux restante, tem-se:

$$
\delta(\langle\Phi, \mathbf{N}-\mathbf{R}\rangle)=\langle\delta \Phi, \mathbf{N}-\mathbf{R}\rangle+\langle\Phi, \mathcal{L} \delta \mathbf{Q}\rangle-\langle\Phi, \mathbf{S} \delta \mathbf{P}\rangle
$$

Para o segundo termo do lado direito da eq. (1.13), ao fazer uso do teorema de Gauss, transferem-se os operadores diferenciais das variáveis de estado $\mathbf{Q}$ para os multiplicadores de Lagrange $\Phi$, o que resulta em:

$$
\langle\Phi, \mathcal{L} \delta \mathbf{Q}\rangle=\left\langle\mathcal{L}^{*} \Phi, \delta \mathbf{Q}\right\rangle-P[\Phi, \delta \mathbf{Q}]_{s}
$$

onde o operador $P[\Phi, \delta \mathbf{Q}]_{s}$ representa o concomitante bilinear (CACUCI et al., 1980; MORSE; FESHBACH, 1953), que recebe este nome porque é linear nas variáveis $\operatorname{adjuntas} \Phi$ e nas variações do escoamento $\delta \mathbf{Q}$. Este termo deve ser avaliado nas fronteiras apropriadas do domínio de interesse, daí o subscrito [ $]_{s}$. Nesse caso, ele dá origem às condições de contorno do problema dual ${ }^{3}$ (HAYASHI, 2009). Já termo $\mathcal{L}^{*}$ representa o operador adjunto de $\mathcal{L}$. A primeira variação do funcional

\footnotetext{
${ }^{2}$ Em mecânica clássica, uma restrição holonômica é aquela que pode ser escrita na forma $f\left(q_{1}, q_{2}, q_{3}, \ldots, q_{n}, t\right)=0$, onde $\left\{q_{1}, q_{2}, q_{3}, \ldots, q_{n}\right\}$ são as coordenadas que descrevem o sistema. As equações que governam o movimento de fluidos também dependem, tipicamente, de termos $\dot{q}_{j}$ e são, geralmente, não-holonômicas.

${ }^{3}$ No contexto de otimização, o problema adjunto é denominado dual, enquanto o problema físico é conhecido por primal.
} 
aumentado $\delta G$, fica então definida pela expressão:

$$
\begin{aligned}
\delta G= & -\langle\delta \Phi, \mathbf{N}-\mathbf{R}\rangle-\langle\delta \Psi, \mathcal{B}\rangle_{s}-\left\langle\mathcal{L}^{*} \Phi-\mathcal{F}_{\mathbf{Q}}^{\prime}, \delta \mathbf{Q}\right\rangle+ \\
& +P[\Phi, \delta \mathbf{Q}]_{s}-\left\langle\Psi, \mathcal{B}_{\mathbf{Q}}^{\prime} \delta \mathbf{Q}\right\rangle_{s}+\left\langle\mathcal{F}_{\mathcal{P}}^{\prime}, \delta \mathcal{P}\right\rangle+\langle\Phi, \mathbf{S} \delta \mathcal{P}\rangle
\end{aligned}
$$

Uma vez que a solução do escoamento esteja convergida, os dois primeiros termos do lado direito da eq. (1.15) são naturalmente nulos. O terceiro e o quarto termos são anulados ao "escolher" $\mathbf{\Phi}$ de forma a satisfazer a equação adjunta:

$$
\mathcal{L}^{*} \Phi=\mathcal{F}_{\mathrm{Q}}^{\prime}
$$

e suas respectivas condições de contorno.

Por fim, da eq. (1.12b), o quinto termo do lado direito da eq. (1.15) também é zero. Logo, a variação da medida de mérito $\delta G$ se restringe à expressão:

$$
\delta G=\left\langle\mathcal{F}_{\mathcal{P}}^{\prime}, \delta \mathcal{P}\right\rangle+\langle\Phi, \mathbf{S} \delta \mathcal{P}\rangle
$$

Portanto, a resolução da equação adjunta (1.16) permite uma simplificação na forma final de $\delta G$, apresentada pela equação (1.17), que é uma conseqüência direta da imposição da realizabilidade de $\delta \mathbf{Q}$ através da eq. (1.12). Note também, que a precisão do gradiente obtido pelo método adjunto não depende mais da magnitude de qualquer variação dos parâmetros $\delta \mathcal{P}_{\alpha}$ como ocorria no caso de diferenças finitas. Ao lado disso, a independência da variação $\delta G$ com relação a $\delta \mathbf{Q}$ permite que se calcule a sensibilidade com respeito a qualquer número de parâmetros $\mathcal{P}_{\alpha}$, sem a necessidade de simulações adicionais do escoamento. Essa é a essência do método adjunto proposto por Jameson (JAMESON, 1988; JAMESON, 1994) para escoamentos transônicos.

Note que ambos os termos $\mathcal{L}^{*}$ (que define os coeficientes das variáveis adjuntas) e $\mathcal{F}_{\mathbf{Q}}^{\prime}$ (termo não-homogêneo) da equação (1.16) são independentes do vetor $\boldsymbol{\Phi}$, o que faz com que a equação adjunta seja linear. É interessante notar que ela seja linear apesar de ser derivada a partir das equações que governam escoamentos que são, geralmente, não-lineares (como no caso das equações de Euler ou Navier-Stokes) e que formulações dos problemas primal e dual são representações equivalentes do mesmo problema linear ${ }^{4}$, como mostram Giles e Pierce (1997). Além disso, sua estrutura concentra no lado esquerdo os termos que dependem apenas das equações que governam o escoamento, enquanto o termo não-homogêneo, no lado direito, é o único que envolve a particular medida de

\footnotetext{
${ }^{4}$ Observe que as eqs. (1.12) representam uma linearização do problema primal (1.10) e que o problema adjunto (dual) advém dessa linearização.
} 
mérito adotada.

Se, por um lado, a linearidade traz benefícios claros para as aplicações; por outro, a estrutura da equação adjunta tem profundas consequências na formulação do método. A separação entre os termos que dependem das equações da mecânica dos fluidos e os que envolvem a medida de mérito implica na existência de um operador adjunto associado apenas ao modelo da física do escoamento. Então, em princípio, ao se obter o operador associado ao modelo em questão, pode-se otimizar uma ampla gama de medidas de mérito, pois isto envolveria modificar apenas o termo não-homogêneo, que por sinal, está presente somente na condição de contorno de parede ${ }^{5}$ e que, consequentemente, consiste em integrais apenas na superfície aerodinâmica que se deseja otimizar. Outra consequência imediata desta separação é que, em grande medida, o algoritmo para resolver a equação (1.16) depende do particular operador adjunto considerado, e não do termo forçante. Isso permite enorme flexibilidade na definição das medidas de mérito.

Vale acrescentar que, no escopo desta tese, as equações adjuntas são obtidas a partir de uma solução estacionária do escoamento. Assim, as variáveis de estado Q são constantes durante o processo de solução do problema dual e, portanto, o termo $\mathcal{L}^{*}$ nada mais é que uma matriz quadrada $4 \times 4$ constante para cada elemento da malha computacional durante o processo de solução do problema adjunto no caso bidimensional, por exemplo. Consequentemente, basta "invertêla" para resolver o sistema de equações (1.16) e obter a solução adjunta.

Embora restritas a problemas mais simples, as soluções analíticas baseadas na função de Green ilustram perfeitamente as propriedades apontadas acima. Com este enfoque, Giles e Pierce conduziram extensa investigação das propriedades matemáticas das equações adjuntas e as funções de Green a elas associadas (GILES; PIERCE, 1997; GILES; PIERCE, 1998a; GILES; PIERCE, 2000b). Obtiveram soluções analíticas para problemas selecionados (GILES; PIERCE, 2000a) e identificaram outras aplicações importantes do método adjunto. Exemplos da variedade de tais aplicações são a estimativa de funcionais de arrasto e sustentação (GILES; PIERCE, 1999a; GILES; PIERCE, 1999b), além da análise numérica em CFD (GILES; PIERCE, 1997; GILES; PIERCE, 1998b).

\footnotetext{
${ }^{5}$ É claro que, nem todas as medidas de mérito relevantes a aplicações aerodinâmicas se restringem a condição de contorno de parede. Entretanto, as funções objetivo tratadas neste trabalho se limitam a forças e momentos, que se enquadram nessa condição.
} 


\subsubsection{Abordagem Alternativa - Cacuci}

Esta seção apresenta um resumo da formulação alternativa do método adjunto proposta por Cacuci et al. (1980) para se obter sensibilidades gerais de PDE's nãolineares. É justamente esta abordagem que permite que se avaliem sensibilidades em relação a parâmetros de projeto impostos nas fronteiras permeáveis onde ocorre entrada de escoamento no domínio computacional. Para isso, considerase inicialmente uma medida de mérito, ou funcional objetivo, de forma genérica dada pela mesma eq. (1.2) utilizada na seção 1.4.2.

Supondo que as equações que governam o escoamento componham um sistema de $K$ equações diferenciais não-lineares $\mathbf{N}$, submetidas a um conjunto de condições de contorno correspondentes $\mathcal{B}$. Em termos de operadores:

$$
\begin{aligned}
& \mathbf{N}[\mathbf{Q}(\mathcal{X}), \mathcal{P}]=\mathbf{R}(\mathcal{X}, \mathcal{P}) \\
& \mathcal{B}[\mathbf{Q}(\mathcal{X}), \mathcal{P}]_{s}=0
\end{aligned}
$$

Note que as eqs. (1.10) e (1.18) diferem apenas por suas condições de contorno (1.10b) e (1.18b). Na última, o operador de condições de contorno passa a depender também dos parâmetros que controlam o sistema.

O funcional aumentado $G$ poderia, então, ser escrito na forma:

$$
G(\mathbf{Q}, \mathcal{P}, \Phi, \Psi, \mathbf{a})=I[\mathbf{Q}, \mathcal{P}]-\langle\Phi, \mathbf{N}-\mathbf{R}\rangle-\langle\Psi, \mathcal{B}\rangle_{s}-\left\langle\mathbf{a}, \mathcal{P}-\mathcal{P}_{o}\right\rangle
$$

onde os vetores $\Phi, \Psi$ e a são multiplicadores de Lagrange. O primeiro impõe as equações que governam o escoamento, e que podem ser vistas como condições de realizabilidade. $\mathrm{O}$ vetor $\Psi$ impõe as condições de contorno correspondentes, e o vetor a impõe que os parâmetros de controle assumam os valores referentes a uma dada configuração base $\mathcal{P}=\mathcal{P}_{o}$.

Considere, agora, as diferenciais de Fréchet das eqs. (1.18a) e (1.18b):

$$
\begin{aligned}
\mathcal{L} \delta \mathbf{Q} & =\mathbf{S} \delta \mathcal{P} \\
\mathcal{B}_{\mathbf{Q}}^{\prime} \delta \mathbf{Q} & =-\mathcal{B}_{\mathcal{P}}^{\prime} \delta \mathcal{P}
\end{aligned}
$$

Vale ressaltar que é justamente neste ponto que reside a principal diferença da formulação proposta por Cacuci et al. (1980) em relação a abordagem de Jameson (1988). Note que ao impor parâmetros de controle $\mathcal{P}$ nas condições de contorno $\mathcal{B}$, sua diferencial de Fréchet passa a ter dois termos $\left(\mathcal{B}_{\mathbf{Q}}^{\prime} \delta \mathbf{Q}=\right.$ $\left.-\mathcal{B}_{\mathcal{P}}^{\prime} \delta \mathcal{P}\right)$ e, consequentemente, a parte física dessa variação passa a ser não-nula, 
ao contrário da formulação tradicional $\left(\mathcal{B}_{\mathbf{Q}}^{\prime} \delta \mathbf{Q}=0\right)$ abordada na seção anterior.

Para se calcular a primeira variação de (1.19), observa-se que os dois primeiros componentes de seu lado direito já tem sua variação determinada em (1.7) e (1.13). Avaliando as demais variações de Gâteaux separadamente, tem-se:

$$
\begin{aligned}
\delta(\langle\Psi, \mathcal{B}\rangle) & =\langle\delta \Psi, \mathcal{B}\rangle_{s}+\left\langle\Psi, \mathcal{B}_{\mathbf{Q}}^{\prime} \delta \mathbf{Q}\right\rangle_{s}+\left\langle\Psi, \mathcal{B}_{\mathcal{P}}^{\prime} \delta \mathcal{P}\right\rangle_{s} \\
\delta\left(\left\langle\mathbf{a}, \mathcal{P}-\mathcal{P}_{o}\right\rangle\right) & =\left\langle\delta \mathbf{a}, \mathcal{P}-\mathcal{P}_{o}\right\rangle+\langle\mathbf{a}, \delta \mathcal{P}\rangle
\end{aligned}
$$

A primeira variação do funcional aumentado $\delta G$ fica, então, definida pela expressão:

$$
\begin{aligned}
\delta G= & -\langle\delta \Phi, \mathbf{N}-\mathbf{R}\rangle-\langle\delta \Psi, \mathcal{B}\rangle_{s}-\left\langle\delta \mathbf{a}, \mathcal{P}-\mathcal{P}_{o}\right\rangle-\left\langle\mathcal{L}^{*} \Phi-\mathcal{F}_{\mathbf{Q}}^{\prime}, \delta \mathbf{Q}\right\rangle+ \\
& -\left\langle\Psi, \mathcal{B}_{\mathbf{Q}}^{\prime} \delta \mathbf{Q}\right\rangle_{s}+P[\Phi, \delta \mathbf{Q}]_{s}+\left\langle\mathcal{F}_{\mathcal{P}}^{\prime}, \delta \mathcal{P}\right\rangle+\langle\Phi, \mathbf{S} \delta \mathcal{P}\rangle-\langle\mathbf{a}, \delta \mathcal{P}\rangle+ \\
& -\left\langle\Psi, \mathcal{B}_{\mathcal{P}}^{\prime} \delta \mathcal{P}\right\rangle_{s}
\end{aligned}
$$

Quando não se tinham parâmetros de controle na fronteira, o termo $\left\langle\Psi, \mathcal{B}_{\mathbf{Q}}^{\prime} \delta \mathbf{Q}\right\rangle_{s}$ era, naturalmente, nulo. Entretanto, com $\mathcal{B}$ dependendo dos parâmetros $\mathcal{P}$, isto não é mais verdade. Assim, para eliminar esse termo da variação total, Cacuci et al. (1980) propõe uma decomposição do concomitante bilinear $P[\Phi, \delta \mathbf{Q}]_{s}$ em dois termos. Ambos são produtos internos, que devem ser computados sobre as fronteiras apropriadas. O primeiro deles envolve um operador $P_{1}(\Phi)$ e a forma linearizada do operador de condições de contorno do escoamento $\mathcal{B}_{\mathbf{Q}}^{\prime} \delta \mathbf{Q}$. Enquanto o segundo envolve $\mathcal{B}^{*}(\Phi)$, que representa o operador das condições de contorno adjuntas, e o termo $\mathcal{M} \delta \mathbf{Q}$. A decomposição de $P$ não é única, e o mesmo vale para a definição de $P_{1}$ e $\mathcal{M}$. Ao contrário, a única restrição que efetivamente se impõe no processo é que o operador $\mathcal{M}$ seja linearmente independente de $\mathcal{B}_{\mathbf{Q}}^{\prime}$. Como resultado, a própria determinação do operador de condições de contorno adjuntas depende de uma decomposição que não é única, e faz sentido que seja assim. Afinal, deve haver alguma flexibilidade no método, para garantir que o problema adjunto possa ser bem posto. Assim, a eq. (1.23) pode ser escrita na forma:

$$
\begin{aligned}
\delta G= & -\langle\delta \Phi, \mathbf{N}-\mathbf{R}\rangle-\langle\delta \Psi, \mathcal{B}\rangle_{s}-\left\langle\delta \mathbf{a}, \mathcal{P}-\mathcal{P}_{o}\right\rangle+\left\langle\mathcal{L}^{*} \Phi-\mathcal{F}_{\mathbf{Q}}^{\prime}, \delta \mathbf{Q}\right\rangle+ \\
& -\left\langle\Psi, \mathcal{B}_{\mathbf{Q}}^{\prime} \delta \mathbf{Q}\right\rangle_{s}-\left[\left\langle P_{1}(\Phi), \mathcal{B}_{\mathbf{Q}}^{\prime} \delta \mathbf{Q}\right\rangle_{s}+\left\langle\mathcal{B}^{*}(\Phi), \mathcal{M} \delta \mathbf{Q}\right\rangle_{s}\right]+\left\langle\mathcal{F}_{\mathcal{P}}^{\prime}, \delta \mathcal{P}\right\rangle+ \\
& +\langle\Phi, \mathbf{S} \delta \mathcal{P}\rangle-\langle\mathbf{a}, \delta \mathcal{P}\rangle-\left\langle\Psi, \mathcal{B}_{\mathcal{P}}^{\prime} \delta \mathcal{P}\right\rangle_{s}
\end{aligned}
$$

Extremos do funcional aumentado $G$ são obtidos desde que (1.24) seja zero 
para variações arbitrárias, porém realizáveis, de seus parâmetros. Isto é:

$$
\delta G=0 \forall\{\delta \mathbf{Q}, \delta \mathcal{P}, \delta \Phi, \delta \Psi, \delta \mathbf{a}\} \in\{\text { locus de realizabilidade }\}
$$

Tal ocorrência, por sua vez, implica que as seguintes condições sejam satisfeitas:

I - As equações que governam o escoamento (1.18a) e as repectivas condições de contorno (1.18b) devem ser satisfeitas. Além disso, os parâmetros de controle devem corresponder à configuração base, $\mathcal{P}=\mathcal{P}_{o}$. Isso implica que os três primeiros termos do lado direito da eq. (1.24) sejam identicamente nulos.

II - Quando se impõe que

$$
\Psi=-P_{1}(\Phi)
$$

a soma do quinto com o sexto termos do lado direito de (1.24) se anula. Esta condição também resolve os multiplicadores $\Psi$ em termos de $\Phi$.

III - O vetor $\Phi$ deve satisfazer a equação adjunta definida por:

$$
\mathcal{L}^{*} \Phi-\mathcal{F}_{\mathrm{Q}}^{\prime}=0
$$

que aparece no quarto termo de (1.24). As condições de contorno adjuntas são dadas por

$$
\mathcal{B}^{*}(\Phi)=0
$$

que vêm do sétimo termo daquela equação. Estas condições devem determinar o vetor $\Phi$ na fronteira, assim como os valores de $\Psi$ correspondentes.

IV - O vetor a é especificado pela seguinte condição:

$$
\langle\mathbf{a}, \delta \mathcal{P}\rangle=\left\langle\mathcal{F}_{\mathcal{P}}^{\prime}, \delta \mathcal{P}\right\rangle+\langle\Phi, \mathbf{S} \delta \mathcal{P}\rangle-\left\langle\Psi, \mathcal{B}_{\mathcal{P}}^{\prime} \delta \mathcal{P}\right\rangle_{s}
$$

que reune as variações restantes no caso em que $\delta G=0$. De fato, esta é a parte realizável do gradiente de sensibilidade $\delta I$.

Afim de provar a asserção a respeito da sensibilidade (CACUCI et al., 1980), é suficiente identificar que: se as equações governantes (1.18a) e (1.18b) forem identicamente satisfeitas para uma dada variação $\Delta G$ de qualquer magnitude, 
então, da própria definição de $G$ dada pela eq. (1.19), tem-se:

$$
\begin{aligned}
& \Delta G=\Delta I-\langle\mathbf{a}, \Delta \mathcal{P}\rangle \\
& \operatorname{para}\left\{\begin{array}{l}
\Delta G=G\left(\mathbf{Q}_{2}, \mathcal{P}_{2} ; \phi_{2}, \psi_{2}, \mathbf{a}_{2}\right)-G\left(\mathbf{Q}_{1}, \mathcal{P}_{1} ; \phi_{1}, \psi_{1}, \mathbf{a}_{1}\right) \\
\Delta I=I\left(\mathbf{Q}_{2}, \mathcal{P}_{2}\right)-I\left(\mathbf{Q}_{1}, \mathcal{P}_{1}\right) \\
\Delta \mathcal{P}=\mathcal{P}_{2}-\mathcal{P}_{1}
\end{array}\right.
\end{aligned}
$$

Em particular, para uma variação infinitesimal $\Delta G \rightarrow \delta G$, sob as condições acima e onde $\phi, \psi$ e $\mathcal{P}$ satisfazem os 4 requisitos acima, deve corresponder a um valor estacionário de $G$. Portanto, pode-se escrever:

$$
\begin{aligned}
\delta G & =\delta I-\langle\mathbf{a}, \delta \mathcal{P}\rangle=0 \\
\delta I & =\langle\mathbf{a}, \delta \mathcal{P}\rangle \\
\delta I & =\left\langle\mathcal{F}_{\mathcal{P}}^{\prime}, \delta \mathcal{P}\right\rangle+\left\langle\phi,\left(\mathbf{R}_{\mathcal{P}}^{\prime}-\mathbf{N}_{\mathcal{P}}^{\prime}\right) \delta \mathcal{P}\right\rangle+\left\langle P_{1}(\phi), \mathbf{B}_{\mathcal{P}}^{\prime} \delta \mathcal{P}\right\rangle_{s}
\end{aligned}
$$

onde as equações (1.26), (1.29) e a definição de $\mathbf{S}$ foram utilizadas. Com a expressão (1.31) acima, pode-se estimar o gradiente apenas com base na solução adjunta $\phi$ e variações de parâmetros $\delta \mathcal{P}$.

Vale notar que toda variação física $\delta \mathbf{Q}$ foi removida com sucesso da expressão do gradiente. Mais do que isso, o primeiro termo do lado direito da eq. (1.31) é precisamente $\delta I_{\mathcal{P}}$, enquanto o segundo mede os efeitos diretos de $\delta \mathcal{P}$ nas equações governantes e o terceiro faz o mesmo com respeito às suas condições de contorno.

\subsection{Histórico de Pesquisas}

O Núcleo de Dinâmica e Fluidos (NDF-EPUSP) iniciou investigação nessa área com a participação no Projeto FAPESP 2000/13768-4 (VOLPE, 2004; VOLPE, 2005). Trabalhando juntamente com pesquisadores da EMBRAER e da ESSS, desenvolveu-se uma rotina de projeto inverso aerodinâmico baseada no método MGM (VOLPE et al., 2007), que se aplica ao regime de escoamento transônico e tem como finalidade obter uma geometria que aproxime a distribuição de pressões desejada, especificada a priori.

A continuidade das pesquisas nessa área passa, claramente, por métodos que utilizem um modelo mais realista das equações que governam o escoamento. As características descritas nas seções anteriores tornaram o método adjunto uma escolha natural. Volpe e Santos (2009) iniciaram as pesquisas sobre o tema ao desenvolver um procedimento de obtenção de condições de contorno adjuntas baseadas em características que tornam o problema adjunto matematicamente 
tão bem posto quanto o problema primal. Este procedimento ainda possibilita dar um significado físico às variáveis adjuntas, que podem ser entendidas como forças de vínculo generalizadas que mantêm as soluções dentro de um espaço realizável. Utilizando o modelo Euler compressível para escoamentos em bocais quase 1-D, este mesmo trabalho apresenta condições internas, mostrando que o comportamento da solução adjunta ao atravessar ondas de choque depende da natureza da particular medida de mérito adotada.

Essa investigação foi ampliada com os trabalhos de Ceze (2008), Hayashi (2009) e Chieregatti (2008) com o desenvolvimento de rotinas de projeto inverso de aerofólios utilizando o método adjunto baseado nas equações de Euler compressíveis. O trabalho de Hayashi (2009), em particular, estendeu o procedimento de obtenção de condições de contorno adjuntas proposto por Volpe e Santos (2009) em escoamentos quase unidimensionais para problemas multidimensionais. Assim, o problema de contorno adjunto foi construído à imagem e semelhança das equações de Euler 2-D, com ambos os problemas de contorno baseados em relações diferenciais características e variáveis de Riemann, garantindo a compatibilidade entre os problemas primal e dual.

As superfícies aerodinâmicas desses trabalhos foram parametrizadas utilizando o método CST proposto por Kulfan e Bussoletti (2006). As soluções físicas e adjuntas são sempre obtidas pelas mesmas malhas não estruturadas com elementos triangulares. Além disso, ambos os códigos numéricos fazem uso do método de volumes finitos, utilizando a discretização centrada de Jameson, Schmidt e Turkel (1981) com dissipação artificial e integrados no tempo pelo esquema de Runge-Kutta de 5 passos e $2^{\text {a }}$ ordem de precisão.

A título de ilustração, o texto que segue apresenta alguns resultados obtidos nesses trabalhos. Como já dito, em problemas inversos existe a possibilidade de que a distribuição de pressão desejada não seja realizável. Esta dificuldade pode ser contornada tratando o problema como um caso particular de um problema de otimização, com uma função de mérito que mede o erro na solução do problema inverso. Desta forma, o processo deve levar até a solução realizável mais próxima do desejado, no sentido dos mínimos quadrados. Assim, converte-se um problema possivelmente mal-posto em um problema bem-posto (JAMESON, 2003). Por exemplo, se $p_{d}$ é a pressão de superfície desejada, pode-se tomar a função de mérito $I$ como sendo a integral:

$$
I=\frac{1}{2} \int_{b_{w}}\left(p-p_{d}\right)^{2} \mathrm{~d} s
$$


Este funcional, em particular, pode ser muito útil na validação do código computacional implementado. Como as variáveis adjuntas não tem um significado físico direto, é impossível visualizar o campo de solução adjunta e dizer se está correto. Uma maneira de validação do método é utilizar uma medida de mérito como a apresentada pela eq. (1.32) em que a distribuição de pressão objetivo corresponde a uma geometria conhecida. Assim, o código pode ser considerado validado se recuperar a essa geometria. A figura 1.1 apresenta um caso de validação que utiliza essa idéia. Neste caso, a distribuição de pressão desejada corresponde ao escoamento Euler incidindo em um perfil RAE 2822 com número de Mach do escoamento livre $M_{\infty}=0.75$ e ângulo de ataque $1^{\circ}$. Partindo de uma geometria inicial NACA 0012 obteve-se, após 50 ciclos de projeto, o aerofólio delimitado pela linha verde na figura 1.1(b). Apesar de não ser um caso prático de aplicação, já que um projetista jamais gostaria de ter um resultado com uma onda de choque mais forte do que sua forma inicial, este caso destaca uma das qualidades do método adjunto quando aplicado a escoamentos compressíveis: a capacidade de posicionar ondas de choque corretamente.

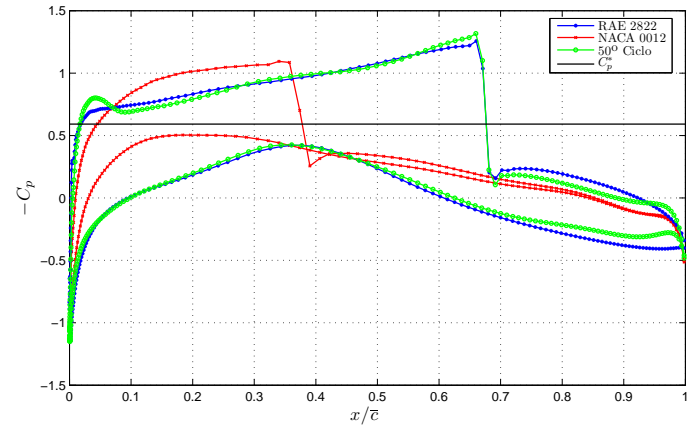

(a) Evolução de $C_{p}$.

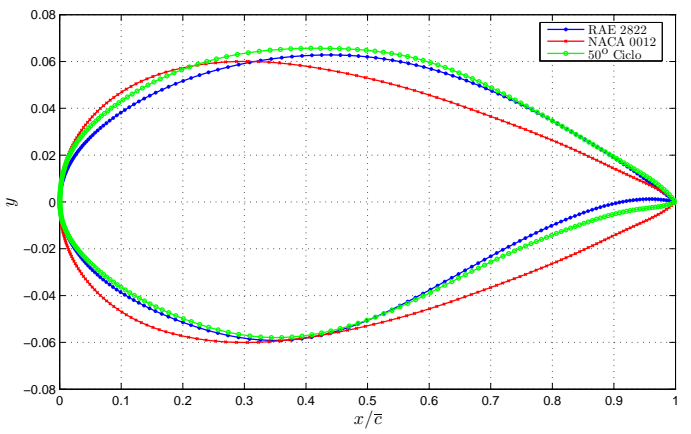

(b) Evolução da geometria.

Figura 1.1: Teste de validação de projeto inverso aerodinâmico $\left(M_{\infty}=0.75\right.$, $\theta=1^{\circ}$ ) - Vermelho, NACA 0012 (configuração inicial); azul, RAE 2822 (objetivo); verde, resultado final.

Já a figura 1.2 representa um caso de aplicação um pouco mais realista, em que o perfil inicial é o RAE 2822 e as condições de escoamento livre são as mesmas do caso anterior. A distribuição desejada, agora, não corresponde a uma geometria pré-definida, ela foi editada visando a eliminação da onda de choque. Aqui, vale notar que a edição da distribuição objetivo não garante que esta seja realizável. Entretanto, ao formular o problema de maneira apropriada, ou seja, como um caso especial de otimização, o funcional é minimizado e, apesar de não conseguir eliminar a onda de choque, minimizou-se a diferença entre a distribuição de pressão inicial e a desejada. Além disso, características como o chamado roof top 
foram alcançadas com sucesso.

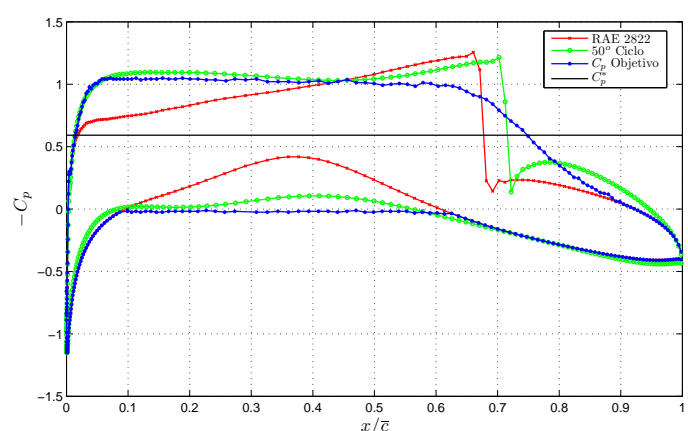

(a) Evolução de $C_{p}$.

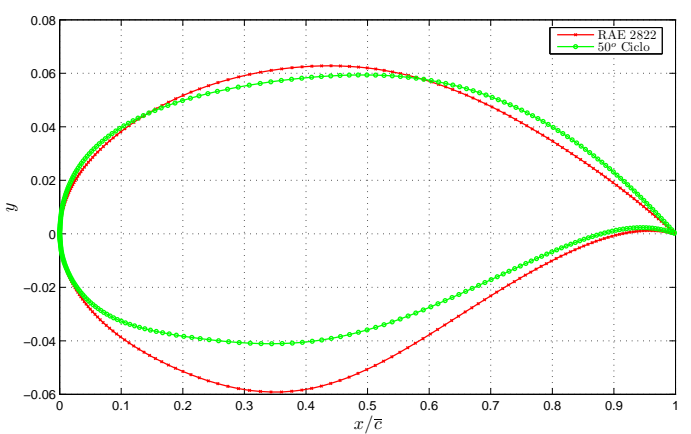

(b) Evolução da geometria.

Figura 1.2: Exemplo de aplicação de projeto inverso aerodinâmico $\left(M_{\infty}=0.75\right.$, $\theta=1^{\circ}$ ) - Vermelho, RAE 2822 (configuração inicial); azul, objetivo; verde, resultado final.

\subsection{Objetivos, Contribuição e Estrutura da Tese}

Este trabalho tem por objetivo estender a capacidade de avaliar sensibilidades do método adjunto, ao desenvolver a formulação contínua do método aplicada às equações de Euler compressíveis, utilizando a abordagem originalmente proposta por Cacuci et al. (1980) para obtenção de sensibilidades gerais de sistemas de PDE's não-lineares, que foi apresentada, em linhas gerais, na seção 1.4.3. Além disso, também visa mostrar que as condições de contorno adjuntas propostas por Hayashi (2009) são compatíveis com a decomposição do concomitante bilinear sugerida por Cacuci et al. (1980) e que permitem, portanto, a obtenção de gradientes de medidas de mérito com relação a variáveis físicas do escoamento em fronteiras permeáveis do domínio computacional como a de entrada de escoamento. Desta forma, é possível, por exemplo, estimar sensibilidades de forças como sustentação e arrasto com relação a parâmetros como velocidade, pressão estática e massa específica na condição de escoamento não perturbado. Mais do que isso, esta informação pode ser computada a partir da mesma solução adjunta utilizada para otimização de forma. Esse conceito deve, em princípio, permitir que se utilize o método adjunto para a obtenção de derivadas de estabilidade de aeronaves a partir de uma solução estacionária do escoamento.

É claro que o escopo deste trabalho se limita a apresentar uma prova de conceito, dadas as limitações de tempo inerentes a uma tese de doutorado. Nesse contexto, é natural a escolha de um modelo matemático simplificado para representar o movimento de fluidos. Apesar de representarem um modelo idealizado 
do escoamento, as equações de Euler, apresentadas no Capítulo 2, retratam uma parte importante da física em aplicações típicas de engenharia aeronáutica, o que justifica a sua escolha. O Capítulo 3 expõe, detalhadamente, a derivação do método adjunto aplicado às equações de Euler e seu problema de contorno. O Capítulo 4 expõe os resultados de validação, que comparam os gradientes obtidos através do método adjunto e por diferenças finitas em casos-teste que incluem escoamentos internos e externos tanto nos regimes subsônico, como supersônico com diferentes medidas de mérito. Por fim, o Capítulo 5 apresenta as conclusões e sugestões para trabalhos futuros. 


\section{Modelo Matemático do ESCOAMENTO}

Este capítulo tem por objetivo apresentar o modelo matemático escolhido para representar as equações que governam o escoamento. Em particular, dois conceitos importantes são expostos no que segue: sua formulação característica, que é fundamental para o problema de contorno, e uma transformação do sistema de coordenadas cartesiano para o generalizado, que será primordial para a derivação das equações adjuntas no capítulo 3 .

\subsection{As Equações de Navier-Stokes}

Sabe-se que o movimento dos fluidos compressíveis é completamente descrito pelas leis de conservação de massa, quantidade de movimento e energia (HIRSCH, 1988a). Dentre os modelos matemáticos utilizados para representar essas leis, aquele que as descreve com o maior nível de aproximação da física do escoamento é representado pelo sistema de equações (2.1) abaixo em sua forma integral.

$$
\begin{aligned}
\text { massa } & \frac{\partial}{\partial t} \int_{\mathcal{V}} \rho \mathrm{d} \mathcal{V}+\oint_{\mathcal{S}} \rho \overrightarrow{\mathbf{v}} \cdot \mathrm{d} \overrightarrow{\mathcal{S}}=0 \\
\text { quant. mov. } & \frac{\partial}{\partial t} \int_{\mathcal{V}} \rho \overrightarrow{\mathbf{v}} \mathrm{d} \mathcal{V}+\oint_{\mathcal{S}}(\rho \overrightarrow{\mathbf{v}} \cdot \mathrm{d} \overrightarrow{\mathcal{S}}) \overrightarrow{\mathbf{v}}+\oint_{\mathcal{S}} p \mathrm{~d} \overrightarrow{\mathcal{S}}=\oint_{\mathcal{S}} \overrightarrow{f_{e}} \mathrm{~d} \mathcal{S} \\
\text { energia } & \frac{\partial}{\partial t} \int_{\mathcal{V}} e \mathrm{~d} \mathcal{V}+\oint_{\mathcal{S}} \rho H \overrightarrow{\mathbf{v}} \cdot \mathrm{d} \overrightarrow{\mathcal{S}}=\oint_{\mathcal{S}} \vec{f}_{e} \cdot \overrightarrow{\mathbf{v}} \mathrm{d} \mathcal{S}+\oint_{\mathcal{S}} \vec{q} \cdot \mathrm{d} \overrightarrow{\mathcal{S}}
\end{aligned}
$$

Onde $\rho$ é a massa específica de um fluido com velocidade $\overrightarrow{\mathbf{v}}$, sob pressão $p$, sujeito à forças externas $\vec{f}_{e}$, fluxo de calor $\vec{q}$, com entalpia total $H$ e energia específica e em um domínio de volume $\mathcal{V}$ e área de superfície $\mathcal{S}$.

Observa-se que, no caso tridimensional, por exemplo, este sistema possui 15 incógnitas (massa específica $\rho, 3$ componentes do vetor velocidade $\overrightarrow{\mathbf{v}}$, pressão estática $p, 6$ componentes do tensor de tensões viscosas $\tau$, energia $e$ e 3 componentes 
de fluxo de calor) e apenas 5 equações (conservação de massa, 3 componentes da conservação de quantidade de movimento e conservação de energia). Portanto, este sistema de equações é "aberto", uma vez que existem mais incógnitas do que equações. Assim, é necessário complementá-las com um conjunto de relações constitutivas que permitam "fechá-lo". Este sistema de equações completo, incluindo as relações constitutivas, é freqüentemente referenciado na engenharia aeronáutica como "equações de Navier-Stokes", embora este termo tenha sido empregado, originalmente, apenas à equação de conservação da quantidade de movimento, em homenagem à Claude-Louis Navier e George Gabriel Stokes, primeiros a deduzi-la.

\subsection{As Equações de Euler}

As equações de Euler, deduzidas diretamente das leis de Newton, estão reproduzidas no sistema de equações (2.2) também em sua forma integral. Elas correspondem a uma simplificação das equações de (2.1) e representam um nível de aproximação da física do escoamento em que as forças viscosas e os efeitos de transferência de calor são desprezados. Formalmente, elas são obtidas tomandose o "limite matemático" das equações de Navier-Stokes quando o número de Reynolds tende a infinito (AZEVEDO, 2006), ou seja, quando as forças viscosas são desprezíveis se comparadas às forças inerciais. Apesar de representarem um escoamento idealizado, elas são muito importantes na engenharia aeronáutica, já que grande parte dos escoamentos de interesse na área são de alta velocidade, nos quais as forças viscosas ficam concentradas numa região limitada do domínio conhecida como camada limite. Além disso, são capazes de capturar efeitos de compressibilidade não-lineares como as ondas de choque, fato de extrema relevância quando se fala em projeto aerodinâmico para escoamentos em regime transônico. Desta forma, acredita-se que elas são suficientes para primeira aplicação da formulação adjunta utilizada para a obtenção do gradiente de sensibilidades não-geométricos proposto por este trabalho.

$$
\begin{aligned}
& \frac{\partial}{\partial t} \int_{\mathcal{V}} \rho \mathrm{d} \mathcal{V}+\oint_{\mathcal{S}} \rho \overrightarrow{\mathbf{v}} \cdot \mathrm{d} \overrightarrow{\mathcal{S}}=0 \\
& \frac{\partial}{\partial t} \int_{\mathcal{V}} \rho \overrightarrow{\mathbf{v}} \mathrm{d} \mathcal{V}+\oint_{\mathcal{S}}(\rho \overrightarrow{\mathbf{v}} \cdot \mathrm{d} \overrightarrow{\mathcal{S}}) \overrightarrow{\mathbf{v}}+\oint_{\mathcal{S}} p \mathrm{~d} \overrightarrow{\mathcal{S}}=0 \\
& \frac{\partial}{\partial t} \int_{\mathcal{V}} e \mathrm{~d} \mathcal{V}+\oint_{\mathcal{S}} \rho H \overrightarrow{\mathbf{v}} \cdot \mathrm{d} \overrightarrow{\mathcal{S}}=0
\end{aligned}
$$


Observe que as equações de Euler tridimensionais também formam um sistema de equações "aberto". Entretanto, apenas uma relação constitutiva é necessária para "fechá-lo", uma vez que existem 6 incógnitas (massa específica $\rho, 3$ componentes do vetor velocidade $\overrightarrow{\mathbf{v}}$, pressão estática $p$, e energia $e$ ) e as mesmas 5 equações. Esta relação constitutiva é uma equação de estado termodinâmico, no caso, a equação de gás perfeito.

Essa forma das equações (2.2) é particularmente importante quando se deseja resolvê-las numericamente. O método dos volumes finitos, um dos mais utilizados em códigos de CFD, faz uso dessa forma das equações, e a característica essencial dessa formulação reside na presença da integral de superfície e o fato que a variação no tempo das variáveis conservadas dentro do volume só depende dos valores dos fluxos na superfície. Assim, para uma subdivisão arbitrária do volume $\mathcal{V}$, pode-se escrever as leis de conservação para cada subvolume e recuperar as leis de conservação globais ao somar as leis de conservação dos subvolumes. Ao somar as integrais de superfície as contribuições das linhas internas sempre aparecerão 2 vezes, mas com sinais opostos, e serão canceladas na soma. Essa propriedade essencial tem que ser satisfeita pela discretização numérica das contribuições de fluxo de modo que o esquema seja conservativo. Quando isso não ocorre, ou seja, quando após a soma das equações discretizadas em um certo número de elementos adjacentes, a equação resultante ainda contém contribuições de fluxo, a discretização é dita não-conservativa, e as contribuições de fluxo internas aparecem como fontes numéricas nos volumes internos (HIRSCH, 1988a).

As equações de Euler podem, ainda, ser representadas de outras maneiras. A literatura, frequentemente as apresenta em sua forma divergente apresentada no sistema (2.3), que pode ser obtida ao aplicar o teorema de Gauss às integrais de superfície do sistema de equações (2.2), transformando-as em integrais no volume.

$$
\begin{aligned}
& \frac{\partial}{\partial t} \int_{\mathcal{V}} \rho \mathrm{d} \mathcal{V}+\int_{\mathcal{V}} \nabla \cdot(\rho \overrightarrow{\mathbf{v}}) \mathrm{d} \mathcal{V}=0 \\
& \frac{\partial}{\partial t} \int_{\mathcal{V}} \rho \overrightarrow{\mathbf{v}} \mathrm{d} \mathcal{V}+\int_{\mathcal{V}} \nabla \cdot(\rho \overrightarrow{\mathbf{v}} \overrightarrow{\mathbf{v}}) \mathrm{d} \mathcal{V}+\int_{\mathcal{V}} \nabla \cdot p \mathrm{~d} \mathcal{V}=0 \\
& \frac{\partial}{\partial t} \int_{\mathcal{V}} e \mathrm{~d} \mathcal{V}+\int_{\mathcal{V}} \nabla \cdot(\rho H \overrightarrow{\mathbf{v}}) \mathrm{d} \mathcal{V}=0
\end{aligned}
$$

onde o operador divergente $\nabla$, que nomeia esta forma de representação das equa- 
ções, é dado pela seguinte expressão:

$$
\nabla \equiv \hat{\imath} \frac{\partial}{\partial x}+\hat{\jmath} \frac{\partial}{\partial y}+\hat{\kappa} \frac{\partial}{\partial z}
$$

em que $\hat{\imath}, \hat{\jmath}$ e $\hat{\kappa}$ são os versores nas direções coordenadas $x, y$ e $z$, respectivamente.

Observe, no entanto, que a utilização do teorema de Gauss requer que não haja descontinuidades no volume de controle $\mathcal{V}$ (PRAGER, 1961). Assim, o sistema de equações (2.3), a rigor, não é aplicável em ondas de choque. Nestas regiões, vale apenas a solução fraca, que é obtida somente a partir das equações (2.2) ao considerar a onda de choque isolada em um volume de controle e, então, estabelecer a continuidade dos fluxos de massa, quantidade de movimento e energia na superfície desse volume. Este procedimento equivale à imposição das relações de Rankine-Hugoniot (LEVEQUE, 2002).

Uma vez que as equações (2.3) são escritas para um volume arbitrário $\mathcal{V}$, elas devem ser válidas localmente para qualquer ponto do domínio. Isto leva a forma diferencial das leis de conservação (HIRSCH, 2007), apresentada abaixo pela equação (2.5) para escoamentos bidimensionais. Aqui vale esclarecer que as derivações presentes neste trabalho estão restritas ao caso 2-D por uma limitação de tempo e escopo naturais a uma tese de doutoramento. O objetivo principal da pesquisa, no entanto, é propor e validar uma abordagem. Embora restrita a aplicações 2-D neste trabalho, ela pode perfeitamente ser extendida aos casos 3-D, na sua continuação em trabalhos futuros.

$$
\frac{\partial \mathbf{Q}}{\partial t}+\frac{\partial \mathbf{E}}{\partial x}+\frac{\partial \mathbf{F}}{\partial y}=0
$$

onde o vetor de estados $\mathbf{Q}$ e os vetores de fluxo $\mathbf{E}$ e $\mathbf{F}$ são dados por:

$$
\mathbf{Q}=\left\{\begin{array}{c}
\rho \\
\rho u \\
\rho v \\
e
\end{array}\right\} \quad, \quad \mathbf{E}=\left\{\begin{array}{c}
\rho u \\
\rho u^{2}+p \\
\rho u v \\
(e+p) u
\end{array}\right\} \quad, \quad \mathbf{F}=\left\{\begin{array}{c}
\rho v \\
\rho u v \\
\rho v^{2}+p \\
(e+p) v
\end{array}\right\}
$$

De modo a obter a relação constitutiva para completar o sistema de equações é comum, neste tipo de aplicação, utilizar a hipótese de gás termicamente perfeito:

$$
p=\rho R T=(\gamma-1) \rho e_{i}
$$

onde $T$ é a temperatura absoluta do fluido, $\gamma$ é a razão de calores específicos, $R$ 
é a constante do gás e a energia interna específica $e_{i}$ é dada por:

$$
e_{i}=C_{v} T
$$

Como última hipótese, admite-se que o gás seja também caloricamente perfeito, ou seja, os calores específicos e a relação abaixo são constantes:

$$
\gamma \equiv \frac{C_{p}}{C_{v}}=\text { constante }
$$

Por fim, a energia específica total, definida pela soma da energia interna com a energia cinética, é dada pela seguinte expressão:

$$
e \equiv \rho\left[e_{i}+\frac{1}{2}\left(u^{2}+v^{2}\right)\right]
$$

Associando as equações (2.7) e (2.10), obtém-se a equação de estado (2.11) abaixo que será utilizada para "fechar" o sistema:

$$
p=\left[e-\frac{1}{2}\left(\rho u^{2}+\rho v^{2}\right)\right](\gamma-1)
$$

\subsubsection{Forma Característica das Equações de Euler}

Observa-se que todos os autovalores das matrizes jacobianas associadas aos vetores de fluxo nas equações de Euler são reais. Portanto, elas são caracterizadas como um sistema de equações hiperbólicas no domínio espaço-tempo (HIRSCH, 1988a). Além disso, a propagação de informação ocorre por meio das características. Estas propriedades são descritas detalhadamente no que segue.

Considere as equações de Euler bidimensionais em sua forma diferencial dada pela expressão (2.5). Note que os vetores de fluxo $\mathbf{E}$ e $\mathbf{F}$ podem ser escritos em função das variáveis conservadas do vetor de estado Q. Então, pela regra da cadeia:

$$
\frac{\partial \mathbf{E}}{\partial x}=\frac{\partial \mathbf{E}}{\partial \mathbf{Q}} \frac{\partial \mathbf{Q}}{\partial x} \quad, \quad \frac{\partial \mathbf{F}}{\partial y}=\frac{\partial \mathbf{F}}{\partial \mathbf{Q}} \frac{\partial \mathbf{Q}}{\partial y}
$$

Assim, ao substituir as derivadas dos vetores de fluxo, apresentadas acima pelas expressões (2.12), na equação (2.5), obtém-se a forma quase-linear das equações de Euler:

$$
\frac{\partial \mathbf{Q}}{\partial t}+\underbrace{\frac{\partial \mathbf{E}}{\partial \mathbf{Q}}}_{\mathbf{A}} \frac{\partial \mathbf{Q}}{\partial x}+\underbrace{\frac{\partial \mathbf{F}}{\partial \mathbf{Q}}}_{\mathbf{B}} \frac{\partial \mathbf{Q}}{\partial y}=0
$$


onde as matrizes Jacobianas de fluxo A e B são dadas por:

$$
\begin{aligned}
& \mathbf{A}=\left[\begin{array}{cccc}
0 & 1 & 0 & 0 \\
\frac{(\gamma-1)\left(u^{2}+v^{2}\right)}{2}-u^{2} & (3-\gamma) u & (1-\gamma) v & (\gamma-1) \\
-u v & v & u & 0 \\
(\gamma-1) u\left(u^{2}+v^{2}\right)-\gamma u \frac{e}{\rho} & \gamma \frac{e}{\rho}-\frac{(\gamma-1)\left(3 u^{2}+v^{2}\right)}{2} & (1-\gamma) u v & \gamma u
\end{array}\right] \\
& \mathbf{B}=\left[\begin{array}{cccc}
0 & 0 & 1 & 0 \\
-u v & v & u & 0 \\
\frac{(\gamma-1)\left(u^{2}+v^{2}\right)}{2}-v^{2} & (1-\gamma) u & (3-\gamma) v & (\gamma-1) \\
(\gamma-1) v\left(u^{2}+v^{2}\right)-\gamma u \frac{e}{\rho} & (1-\gamma) u v & \gamma \frac{e}{\rho}-\frac{(\gamma-1)\left(u^{2}+3 v^{2}\right)}{2} & \gamma v
\end{array}\right]
\end{aligned}
$$

Aqui, vale ressaltar que $\mathbf{E}$ e $\mathbf{F}$ são homogêneos $^{1}$ de ordem 1 em relação a $\mathbf{Q}$. Desta forma, pode-se mostrar que:

$$
\frac{\partial \mathbf{E}}{\partial \mathbf{Q}}=\mathbf{A} \Rightarrow \mathbf{E}=\mathbf{A Q} \quad \text { e } \quad \frac{\partial \mathbf{F}}{\partial \mathbf{Q}}=\mathbf{B} \Rightarrow \mathbf{F}=\mathbf{B Q}
$$

Observa-se que A e B são complicadas. No entanto, as mesmas equações podem ser escritas em função de um vetor de variáveis primitivas $\mathbf{V}$ (que podem ser medidas diretamente), com matrizes Jacobianas mais simples, como mostra a equação abaixo (HIRSCH, 1988a):

$$
\frac{\partial \mathbf{V}}{\partial t}+\tilde{\mathbf{A}} \frac{\partial \mathbf{V}}{\partial x}+\tilde{\mathbf{B}} \frac{\partial \mathbf{V}}{\partial y}=0
$$

onde:

$$
\mathbf{V}=\left\{\begin{array}{c}
\rho \\
u \\
v \\
p
\end{array}\right\}, \tilde{\mathbf{A}}=\left[\begin{array}{cccc}
u & \rho & 0 & 0 \\
0 & u & 0 & 1 / \rho \\
0 & 0 & u & 0 \\
0 & \gamma p & 0 & u
\end{array}\right], \tilde{\mathbf{B}}=\left[\begin{array}{cccc}
v & 0 & 0 & 0 \\
0 & v & 0 & 0 \\
0 & 0 & v & 1 / \rho \\
0 & 0 & \gamma p & v
\end{array}\right]
$$

A relação entre as matrizes Jacobianas das variáveis conservadas e primitivas é dada pela seguinte transformação de similaridade:

$$
\begin{aligned}
\tilde{\mathbf{A}} & =\mathbf{M}^{-1} \mathbf{A M} \\
\tilde{\mathbf{B}} & =\mathbf{M}^{-1} \mathbf{B M}
\end{aligned}
$$

onde M, matriz Jacobiana da transformação das variáveis conservadas para as

\footnotetext{
${ }^{1}$ Lomax, Pulliam e Zingg (2001) apresentam a propriedade homogênea das equações de Euler de uma forma mais detalhada.
} 
variáveis primitivas, e a sua inversa $\mathbf{M}^{-1}$ são definidas como:

$$
\begin{gathered}
\mathbf{M}=\frac{\partial \mathbf{Q}}{\partial \mathbf{V}}=\left[\begin{array}{cccc}
1 & 0 & 0 & 0 \\
u & \rho & 0 & 0 \\
v & 0 & \rho & 0 \\
\frac{1}{2}\left(u^{2}+v^{2}\right) & \rho u & \rho v & \frac{1}{\gamma-1}
\end{array}\right] \\
\mathbf{M}^{-1}=\frac{\partial \mathbf{V}}{\partial \mathbf{Q}}=\left[\begin{array}{cccc}
1 \\
-\frac{u}{\rho} & 0 & 0 & 0 \\
-\frac{v}{\rho} & 0 & 0 & 0 \\
\frac{1}{2}(\gamma-1)\left(u^{2}+v^{2}\right) & -(\gamma-1) u & -(\gamma-1) v & \gamma-1
\end{array}\right]
\end{gathered}
$$

Naturalmente, existem outras formas de representar as equações de Euler. Pode-se escrevê-las através de qualquer combinação linear destas equações ou, em outras palavras, multiplicando-as por qualquer matriz inversível de mesma dimensão das matrizes Jacobianas. Observe que este procedimento altera as equações, mas não as variáveis dependentes, que ainda são conservadas (LANEY, 1998).

Uma vez que as equações de Euler são hiperbólicas, sempre é possível definir uma matriz $\mathbb{A}_{K}$ associada à um vetor $\vec{k}$ que possui autovalores reais e um conjunto completo de autovetores para qualquer direção deste vetor. Em outras palavras, o caráter hiperbólico das equações de Euler implica que a matriz $\mathbb{A}_{K}$ seja diagonalizável.

$$
\mathbb{A}_{K}=\vec{k} \cdot \overrightarrow{\mathbf{A}}=k_{x} \cdot \mathbf{A}+k_{y} \cdot \mathbf{B}
$$

onde $k_{x}$ e $k_{y}$ são, respectivamente, as componentes $x$ e $y$ do vetor $\vec{k}$ no sistema cartesiano bidimensional. Assim, a matriz $\mathbb{A}_{K}$ pode ser escrita da seguinte forma:

$$
\mathbb{A}_{K}=\left[\begin{array}{cc}
0 & k_{x} \\
k_{x}\left[\frac{(\gamma-1)\left(u^{2}+v^{2}\right)}{2}-u^{2}\right]-k_{y} u v & v k_{y}-(\gamma-3) u k_{x} \\
k_{y}\left[\frac{(\gamma-1)\left(u^{2}+v^{2}\right)}{2}-v^{2}\right]-k_{x} u v & v k_{x}-(\gamma-1) u k_{y} \\
\left(u k_{x}+v k_{y}\right)\left[(\gamma-1)\left(u^{2}+v^{2}\right)-\gamma \frac{e}{\rho}\right] & \gamma \frac{e}{\rho} k_{x}-(\gamma-1)\left[u v k_{y}+\frac{\left(3 u^{2}+v^{2}\right) k_{x}}{2}\right] \\
k_{y} & (\gamma-1) k_{x} \\
u k_{y}-(\gamma-1) v k_{x} & (\gamma-1) k_{y} \\
u k_{x}-(\gamma-3) v k_{y} & \gamma\left(u k_{x}+v k_{y}\right)
\end{array}\right]
$$

Observa-se que, apesar de $\mathbb{A}_{K}$ não ser simétrica, ela tem um conjunto com- 
pleto de autovetores, isto é, possui 4 autovalores reais e 4 autovetores linearmente independentes.

A condição de hiperbolicidade das equações de Euler é expressa pela existência de soluções na forma de onda (HIRSCH, 1988b). Portanto, sua solução pode ser interpretada como uma série de ondas que se propagam no espaço carregando informação do escoamento. À cada autovalor $\sigma(\vec{k})$ da matriz $\mathbb{A}_{K}$ pode-se associar uma superfície característica, normal ao vetor $\vec{k}$, que indica a direção de propagação de uma frente de onda, representando um aspecto de escoamentos invíscidos chamado propagação de perturbações. O estado físico de um dado ponto pode ser interpretado como resultado das quantidades transportadas ao longo das características. Isto implica que o sistema de equações pode ser reformulado como relações diferenciais escritas ao longo da frente de onda ou superfícies características apenas, conhecidas na literatura como formulação característica das equações de Euler. Nesse contexto, os autovalores de $\mathbb{A}_{K}$ representam a velocidade de propagação da onda (HIRSCH, 1988b).

Utilizando a transformação apresentada anteriormente para tornar o sistema de equações função das variáveis primitivas, tem-se:

$$
\tilde{\mathbb{A}}_{K}=\mathbf{M}^{-1} \mathbb{A}_{K} \mathbf{M}
$$

É possível, então, diagonalizar este sistema de equações ao utilizar a seguinte relação:

$$
\begin{aligned}
\boldsymbol{\Lambda} & =\mathbf{L}^{-1} \tilde{\mathbb{A}}_{K} \mathbf{L} \\
& =\mathbf{L}^{-1} \mathbf{M}^{-1} \mathbb{A}_{K} \mathbf{M L}
\end{aligned}
$$

onde $\boldsymbol{\Lambda}$ é uma matriz diagonal e $\mathbf{L}$ é tal que:

$$
\mathbf{L}^{-1}=\left[\begin{array}{cccc}
1 & 0 & 0 & -1 / c^{2} \\
0 & k_{y} & -k_{x} & 0 \\
0 & k_{x} & k_{y} & 1 / \rho c \\
0 & -k_{x} & -k_{y} & 1 / \rho c
\end{array}\right] \quad, \quad \mathbf{L}=\left[\begin{array}{cccc}
1 & 0 & \rho / 2 c & \rho / 2 c \\
0 & k_{y} & k_{x} / 2 & -k_{x} / 2 \\
0 & -k_{x} & k_{y} / 2 & -k_{y} / 2 \\
0 & 0 & \rho c / 2 & \rho c / 2
\end{array}\right]
$$

Assim, definindo as matrizes:

$$
\mathbf{P}=\mathbf{M L} \quad \text { e } \quad \mathbf{P}^{-1}=\mathbf{L}^{-1} \mathbf{M}^{-1}
$$


Que podem ser escritas de modo completo através das expressões:

$$
\begin{gathered}
\mathbf{P}=\left[\begin{array}{cccc}
1 & 0 & \rho / 2 c & \rho / 2 c \\
u & \rho k_{y} & \frac{\rho\left(c k_{x}+u\right)}{2 c} & \frac{\rho\left(u-c k_{x}\right)}{2 c} \\
v & -\rho k_{x} & \frac{\rho\left(c k_{y}+v\right)}{2 c} & \frac{\rho\left(v-c k_{y}\right)}{2 c} \\
\frac{\left(u^{2}+v^{2}\right)}{2} & \rho\left(u k_{y}-v k_{x}\right) & \frac{\rho}{2}\left(\frac{c}{\gamma-1}+\overrightarrow{\mathbf{v}} \cdot \vec{n}+\frac{u^{2}+v^{2}}{2 c}\right) & \frac{\rho}{2}\left(\frac{c}{\gamma-1}-\overrightarrow{\mathbf{v}} \cdot \vec{n}+\frac{u^{2}+v^{2}}{2 c}\right)
\end{array}\right] \\
\mathbf{P}^{-1}=\left[\begin{array}{cccc}
1-\frac{(\gamma-1)\left(u^{2}+v^{2}\right)}{2 c^{2}} & \frac{(\gamma-1) u}{c^{2}} & \frac{(\gamma-1) v}{c^{2}} & \frac{1-\gamma}{c^{2}} \\
\frac{v k_{x}-u k_{y}}{\rho} & \frac{k_{y}}{\rho} & \frac{-k_{x}}{\rho} & 0 \\
\frac{(\gamma-1)\left(u^{2}+v^{2}\right)-2 c\left(u k_{x}+v k_{y}\right)}{2 \rho c} & \frac{c k_{x}-(\gamma-1) u}{\rho c} & \frac{c k_{y}-(\gamma-1) v}{\rho c} & \frac{\gamma-1}{\rho c} \\
\frac{(\gamma-1)\left(u^{2}+v^{2}\right)+2 c\left(u k_{x}+v k_{y}\right)}{2 \rho c} & \frac{-c k_{x}-(\gamma-1) u}{\rho c} & \frac{-c k_{y}-(\gamma-1) v}{\rho c} & \frac{\gamma-1}{\rho c}
\end{array}\right]
\end{gathered}
$$

Então, a matriz $\mathbb{A}_{K}$ pode ser diagonalizada diretamente da seguinte maneira:

$$
\Lambda=\mathbf{P}^{-1} \mathbb{A}_{K} \mathbf{P}
$$

Resultando na matriz diagonal $\Lambda$, que pode ser escrita explicitamente como:

$$
\boldsymbol{\Lambda}=\left[\begin{array}{cccc}
k_{x} u+k_{y} v & 0 & 0 & 0 \\
0 & k_{x} u+k_{y} v & 0 & 0 \\
0 & 0 & k_{x} u+k_{y} v+c & 0 \\
0 & 0 & 0 & k_{x} u+k_{y} v-c
\end{array}\right]
$$

Os dois primeiros autovalores são iguais à componente normal do vetor velocidade, $v_{n}$. Os dois autovalores restantes estão associados à ondas acústicas e são iguais à $v_{n} \pm c$. Então o sinal destes autovalores será determinado pela componente normal da velocidade na superfície de contorno.

Assim, de maneira a obter as equações de Euler decorrentes da diagonalização da matriz Jacobiana numa direção $\vec{k}$, pode-se pré-multiplicar a equação $(2.15)$ por $\mathbf{L}^{-1}$ :

$$
\mathbf{L}^{-1} \frac{\partial \mathbf{V}}{\partial t}+\mathbf{L}^{-1} \tilde{\mathbf{A}} \frac{\partial \mathbf{V}}{\partial x}+\mathbf{L}^{-1} \tilde{\mathbf{B}} \frac{\partial \mathbf{V}}{\partial y}=0
$$

Mas, como $\mathbf{L L}^{-1}=\mathbf{I}$, pode-se introduzi-lo ao sistema de equações sem alterá-lo:

$$
\mathbf{L}^{-1} \frac{\partial \mathbf{V}}{\partial t}+\mathbf{L}^{-1} \tilde{\mathbf{A}} \mathbf{L} \mathbf{L}^{-1} \frac{\partial \mathbf{V}}{\partial x}+\mathbf{L}^{-1} \tilde{\mathbf{B}} \mathbf{L} \mathbf{L}^{-1} \frac{\partial \mathbf{V}}{\partial y}=0
$$

As relações (2.27) remetem à introdução de um novo conjunto de variáveis características W (HIRSCH, 1988b), também conhecidas como variáveis de Rie- 
mann, definidas pela relação de variações $\delta$ :

$$
\delta \mathbf{W}=\mathbf{L}^{-1} \delta \mathbf{V}=\left\{\begin{array}{c}
\delta w_{1} \\
\delta w_{2} \\
\delta w_{3} \\
\delta w_{4}
\end{array}\right\}=\left\{\begin{array}{c}
\delta \rho-\frac{1}{c^{2}} \delta p \\
k_{y} \delta u-k_{x} \delta v \\
\frac{1}{\rho c} \delta p-\left(k_{x} \delta u+k_{y} \delta v\right) \\
\frac{1}{\rho c} \delta p+\left(k_{x} \delta u+k_{y} \delta v\right)
\end{array}\right\}
$$

Apesar de $\mathbf{L}$ também ser uma matriz Jacobiana, esta não apresenta a propriedade homogênea, descrita no início desta seção, então:

$$
\mathbf{L} \equiv \frac{\partial \mathbf{V}}{\partial \mathbf{W}} \quad \operatorname{mas} \quad \mathbf{V} \neq \mathbf{L} \mathbf{W}
$$

Assim, as variáveis características só são definidas na forma diferencial (2.28). Ao utilizá-las para escrever o sistema de equações características, obtém-se:

$$
\frac{\partial \mathbf{W}}{\partial t}+\mathbf{L}^{-1} \tilde{\mathbf{A}} \mathbf{L} \frac{\partial \mathbf{W}}{\partial x}+\mathbf{L}^{-1} \tilde{\mathbf{B}} \mathbf{L} \frac{\partial \mathbf{W}}{\partial y}=0
$$

Que também pode ser apresentado com os termos expandidos, em função das variáveis primitivas, da seguinte forma:

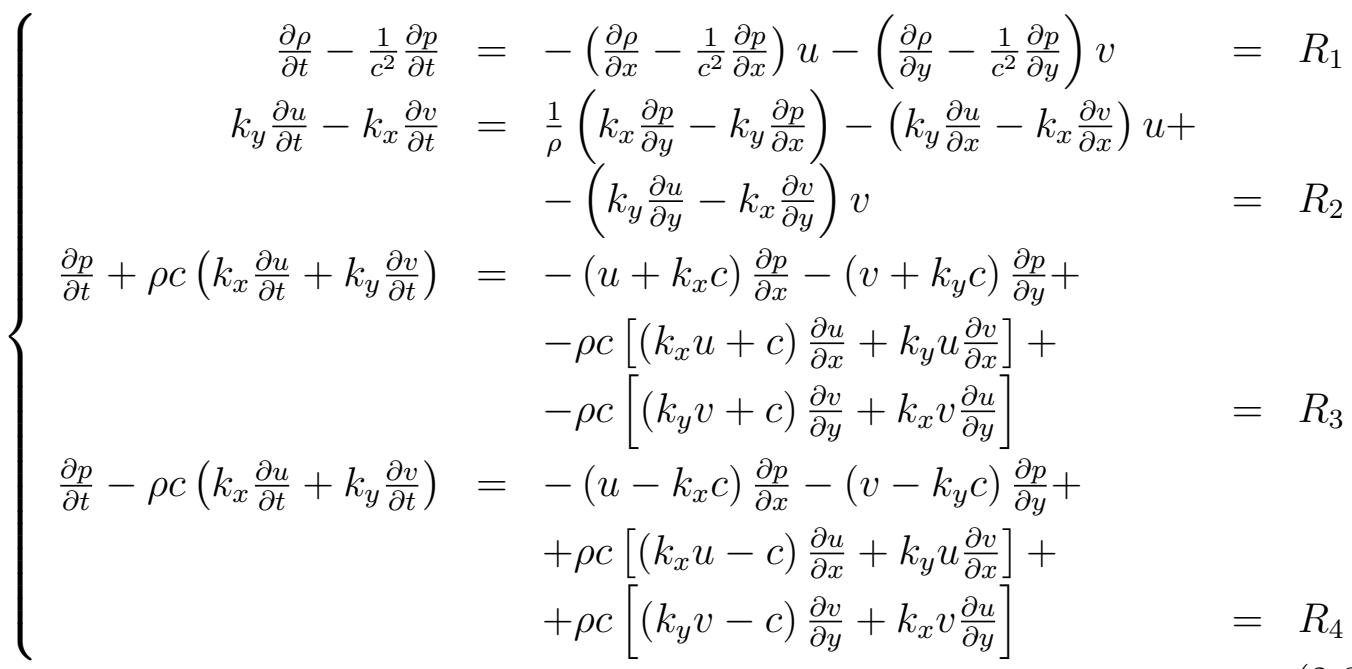

A formulação característica das equações de Euler é completamente equivalente à equação (2.5) apresentada anteriormente. A única diferença entre elas é o fato da formulação característica ter cada uma das equações relacionada a um autovalor (associado a uma direção de propagação $\vec{k}$ ). Assim, esta formulação é obtida de tal modo que as características sejam desacopladas. Em outras palavras, cada equação do sistema (2.31) corresponde, agora, à uma característica. 


\subsubsection{Equações de Euler em Coordenadas Generalizadas}

Considere as equações de Euler bidimensionais em sua forma diferencial escritas em coordenadas cartesianas (2.5). Assumindo uma transformação de coordenadas que transforma o espaço cartesiano em um espaço transformado onde a fronteira do corpo é mapeada em um plano de coordenada constante. Por exemplo, um aerofólio, como indicado na figura 2.1:

$$
\left\{\begin{array}{l}
\tau=t \\
\xi=\xi(x, y, t) \\
\eta=\eta(x, y, t)
\end{array}\right.
$$
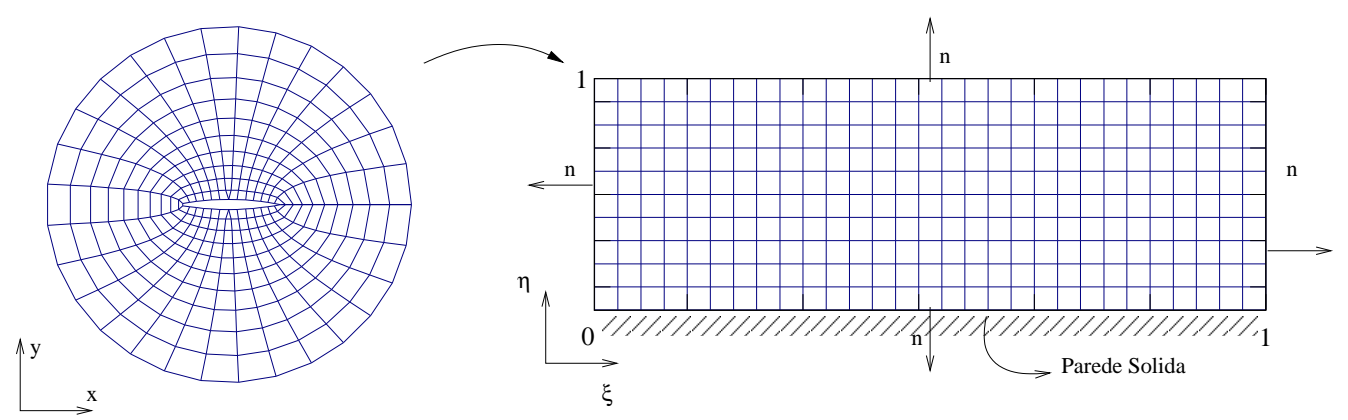

Figura 2.1: Transformação de coordenadas

Então, utilizando a regra da cadeia, pode-se escrever:

$$
\begin{aligned}
\frac{\partial \mathbf{Q}}{\partial t} & =\frac{\partial \mathbf{Q}}{\partial \tau} \frac{\partial f^{1}}{\partial t}+\frac{\partial \mathbf{Q}}{\partial \xi} \frac{\partial \xi}{\partial t}+\frac{\partial \mathbf{Q}}{\partial \eta} \frac{\partial \eta}{\partial t}=\frac{\partial \mathbf{Q}}{\partial \tau}+\xi_{t} \frac{\partial \mathbf{Q}}{\partial \xi}+\eta_{t} \frac{\partial \mathbf{Q}}{\partial \eta} \\
\frac{\partial \mathbf{E}}{\partial x} & =\frac{\partial \mathbf{E}}{\partial \tau} \frac{\partial f^{0}}{\partial x}+\frac{\partial \mathbf{E}}{\partial \xi} \frac{\partial \xi}{\partial x}+\frac{\partial \mathbf{E}}{\partial \eta} \frac{\partial \eta}{\partial x}=\xi_{x} \frac{\partial \mathbf{E}}{\partial \xi}+\eta_{x} \frac{\partial \mathbf{E}}{\partial \eta} \\
\frac{\partial \mathbf{F}}{\partial y} & =\frac{\partial \mathbf{F}}{\partial \tau} \frac{\partial f^{0}}{\partial y}+\frac{\partial \mathbf{F}}{\partial \xi} \frac{\partial \xi}{\partial y}+\frac{\partial \mathbf{F}}{\partial \eta} \frac{\partial \eta}{\partial y}=\xi_{y} \frac{\partial \mathbf{F}}{\partial \xi}+\eta_{y} \frac{\partial \mathbf{F}}{\partial \eta}
\end{aligned}
$$

Substituindo (2.32), (2.33) e (2.34) em (2.5), obtém-se:

$$
\frac{\partial \mathbf{Q}}{\partial \tau}+\xi_{t} \frac{\partial \mathbf{Q}}{\partial \xi}+\eta_{t} \frac{\partial \mathbf{Q}}{\partial \eta}+\xi_{x} \frac{\partial \mathbf{E}}{\partial \xi}+\eta_{x} \frac{\partial \mathbf{E}}{\partial \eta}+\xi_{y} \frac{\partial \mathbf{F}}{\partial \xi}+\eta_{y} \frac{\partial \mathbf{F}}{\partial \eta}=0
$$

Multiplicando (2.35) pelo Jacobiano da transformação $J$, tem-se:

$$
\underbrace{J \frac{\partial \mathbf{Q}}{\partial \tau}}_{I}+\underbrace{J \xi_{t} \frac{\partial \mathbf{Q}}{\partial \xi}}_{I I}+\underbrace{J \eta_{t} \frac{\partial \mathbf{Q}}{\partial \eta}}_{I I I}+\underbrace{J \xi_{x} \frac{\partial \mathbf{E}}{\partial \xi}}_{I V}+\underbrace{J \eta_{x} \frac{\partial \mathbf{E}}{\partial \eta}}_{V}+\underbrace{J \xi_{y} \frac{\partial \mathbf{F}}{\partial \xi}}_{V I}+\underbrace{J \eta_{y} \frac{\partial \mathbf{F}}{\partial \eta}}_{V I I}=0
$$


onde,

$$
J=\left|\frac{\partial(t, x, y)}{\partial(\tau, \xi, \eta)}\right|=\left|\begin{array}{ccc}
1 & x_{\tau} & y_{\tau} \\
0 & x_{\xi} & y_{\xi} \\
0 & x_{\eta} & y_{\eta}
\end{array}\right|=x_{\xi} y_{\eta}-x_{\eta} y_{\xi}
$$

Mas, pela regra da cadeia:

$$
\begin{aligned}
I & \rightarrow \frac{\partial}{\partial \tau}(J \mathbf{Q})=\mathbf{Q} \frac{\partial}{\partial \tau}(J)+J \frac{\partial \mathbf{Q}}{\partial \tau} \\
I I & \rightarrow \frac{\partial}{\partial \xi}\left(J \xi_{t} \mathbf{Q}\right)=\mathbf{Q} \frac{\partial}{\partial \xi}\left(J \xi_{t}\right)+J \xi_{t} \frac{\partial \mathbf{Q}}{\partial \xi} \\
I I I & \rightarrow \frac{\partial}{\partial \eta}\left(J \eta_{t} \mathbf{Q}\right)=\mathbf{Q} \frac{\partial}{\partial \eta}\left(J \eta_{t}\right)+J \eta_{t} \frac{\partial \mathbf{Q}}{\partial \eta} \\
I V & \rightarrow \frac{\partial}{\partial \xi}\left(J \xi_{x} \mathbf{E}\right)=\mathbf{E} \frac{\partial}{\partial \xi}\left(J \xi_{x}\right)+J \xi_{x} \frac{\partial \mathbf{E}}{\partial \xi} \\
V & \rightarrow \frac{\partial}{\partial \eta}\left(J \eta_{x} \mathbf{E}\right)=\mathbf{E} \frac{\partial}{\partial \eta}\left(J \eta_{x}\right)+J \eta_{x} \frac{\partial \mathbf{E}}{\partial \eta} \\
V I & \rightarrow \frac{\partial}{\partial \xi}\left(J \xi_{y} \mathbf{F}\right)=\mathbf{F} \frac{\partial}{\partial \xi}\left(J \xi_{y}\right)+J \xi_{y} \frac{\partial \mathbf{F}}{\partial \xi} \\
V I I & \rightarrow \frac{\partial}{\partial \eta}\left(J \eta_{y} \mathbf{F}\right)=\mathbf{F} \frac{\partial}{\partial \eta}\left(J \eta_{y}\right)+J \eta_{y} \frac{\partial \mathbf{F}}{\partial \eta}
\end{aligned}
$$

Substituindo (2.38)-(2.44) em (2.36), chega-se a seguinte expressão:

$$
\begin{gathered}
\frac{\partial}{\partial \tau}(J \mathbf{Q})-\mathbf{Q} \frac{\partial}{\partial \tau}(J)+\frac{\partial}{\partial \xi}\left(J \xi_{t} \mathbf{Q}\right)-\mathbf{Q} \frac{\partial}{\partial \xi}\left(J \xi_{t}\right)+\frac{\partial}{\partial \eta}\left(J \eta_{t} \mathbf{Q}\right)+ \\
-\mathbf{Q} \frac{\partial}{\partial \eta}\left(J \eta_{t}\right)+\frac{\partial}{\partial \xi}\left(J \xi_{x} \mathbf{E}\right)-\mathbf{E} \frac{\partial}{\partial \xi}\left(J \xi_{x}\right)+\frac{\partial}{\partial \eta}\left(J \eta_{x} \mathbf{E}\right)-\mathbf{E} \frac{\partial}{\partial \eta}\left(J \eta_{x}\right)+ \\
+\frac{\partial}{\partial \xi}\left(J \xi_{y} \mathbf{F}\right)-\mathbf{F} \frac{\partial}{\partial \xi}\left(J \xi_{y}\right)+\frac{\partial}{\partial \eta}\left(J \eta_{y} \mathbf{F}\right)-\mathbf{F} \frac{\partial}{\partial \eta}\left(J \eta_{y}\right)=0(2.45)
\end{gathered}
$$

Rearranjando os termos de forma conveniente, obtém-se:

$$
\begin{aligned}
\frac{\partial}{\partial \tau}(J \mathbf{Q})+\frac{\partial}{\partial \xi}\left[J\left(\xi_{t} \mathbf{Q}+\xi_{x} \mathbf{E}+\xi_{y} \mathbf{F}\right)\right]+\frac{\partial}{\partial \eta}\left[J\left(\eta_{t} \mathbf{Q}+\eta_{x} \mathbf{E}+\eta_{y} \mathbf{F}\right)\right]+ \\
-\left\{\mathbf{Q}\left[\frac{\partial}{\partial \tau}(J)+\frac{\partial}{\partial \xi}\left(J \xi_{t}\right)+\frac{\partial}{\partial \eta}\left(J \eta_{t}\right)\right]+\mathbf{E}\left[\frac{\partial}{\partial \xi}\left(J \xi_{x}\right)+\frac{\partial}{\partial \eta}\left(J \eta_{x}\right)\right]+\right. \\
\left.+\mathbf{F}\left[\frac{\partial}{\partial \xi}\left(J \xi_{y}\right)+\frac{\partial}{\partial \eta}\left(J \eta_{y}\right)\right]\right\}=0(2.46)
\end{aligned}
$$

As derivadas dos termos em coordenadas cartesianas podem ser expressas em função das derivadas dos termos em coordenadas generalizadas da seguinte 
maneira:

$$
\underbrace{\left\{\begin{array}{c}
\frac{\partial}{\partial t} \\
\frac{\partial}{\partial x} \\
\frac{\partial}{\partial y}
\end{array}\right\}}_{\left[D_{c}\right]}=\underbrace{\left[\begin{array}{ccc}
\frac{\partial f^{1}}{\partial t} & \frac{\partial \xi}{\partial t} & \frac{\partial \eta}{\partial t} \\
\frac{\partial f^{0}}{\partial x} & \frac{\partial \xi}{\partial x} & \frac{\partial \eta}{\partial x} \\
\frac{\partial f^{\prime}}{\partial y} & \frac{\partial \xi}{\partial y} & \frac{\partial \eta}{\partial y}
\end{array}\right]}_{[A]} \underbrace{\left\{\begin{array}{c}
\frac{\partial}{\partial \tau} \\
\frac{\partial}{\partial \xi} \\
\frac{\partial}{\partial \eta}
\end{array}\right\}}_{D_{g}} \Rightarrow\left\{D_{c}\right\}=\left[\begin{array}{ccc}
1 & \xi_{t} & \eta_{t} \\
0 & \xi_{x} & \eta_{x} \\
0 & \xi_{y} & \eta_{y}
\end{array}\right]\left\{D_{g}\right\}
$$

ou, de forma inversa:

$$
\left\{\begin{array}{c}
\frac{\partial}{\partial \tau} \\
\frac{\partial}{\partial \xi} \\
\frac{\partial}{\partial \eta}
\end{array}\right\}=\underbrace{\left[\begin{array}{ccc}
\frac{\partial f^{1}}{\partial \tau} & \frac{\partial x}{\partial \tau} & \frac{\partial y}{\partial \tau} \\
\frac{\partial f^{0}}{\partial \xi} & \frac{\partial x}{\partial \xi} & \frac{\partial y}{\partial \xi} \\
\frac{\partial y^{0}}{\partial \eta} & \frac{\partial x}{\partial \eta} & \frac{\partial y}{\partial \eta}
\end{array}\right]}_{[B]}\left\{\begin{array}{c}
\frac{\partial}{\partial t} \\
\frac{\partial}{\partial x} \\
\frac{\partial}{\partial y}
\end{array}\right\} \Rightarrow\left\{D_{g}\right\}=\left[\begin{array}{ccc}
1 & x_{\tau} & y_{\tau} \\
0 & x_{\xi} & y_{\xi} \\
0 & x_{\eta} & y_{\eta}
\end{array}\right]\left\{D_{c}\right\}
$$

Substituindo (2.48) em (2.47), obtém-se a relação:

$$
\left\{D_{c}\right\}=[A][B]\left\{D_{c}\right\} \Rightarrow[A][B]=[I]
$$

onde $[I]$ é a matriz identidade.

Assim, pode-se afirmar que:

$$
[A]=[B]^{-1}
$$

Calculando-se, agora, $[X]=[B]^{-1}$, tem-se:

$$
[X][B]=[I]
$$

ou, ainda:

$$
\left[\begin{array}{lll}
X_{11} & X_{12} & X_{13} \\
X_{21} & X_{22} & X_{23} \\
X_{31} & X_{32} & X_{33}
\end{array}\right]\left[\begin{array}{lll}
1 & x_{\tau} & y_{\tau} \\
0 & x_{\xi} & y_{\xi} \\
0 & x_{\eta} & y_{\eta}
\end{array}\right]=\left[\begin{array}{ccc}
1 & 0 & 0 \\
0 & 1 & 0 \\
0 & 0 & 1
\end{array}\right]
$$

Resolvendo o sistema, chega-se à seguinte matriz $[X]$

$$
[X]=\left[\begin{array}{ccc}
1 & J^{-1}\left(x_{\eta} y_{\tau}-x_{\tau} y_{\eta}\right) & J^{-1}\left(x_{\tau} y_{\xi}-x_{\xi} y_{\tau}\right) \\
0 & J^{-1} y_{\eta} & -J^{-1} y_{\xi} \\
0 & -J^{-1} x_{\eta} & J^{-1} x_{\xi}
\end{array}\right]
$$


Comparando as matrizes $[X]$ e $[A]$, chega-se as seguintes relações:

$$
\begin{aligned}
\xi_{t} & =-x_{\tau} \xi_{x}-y_{\tau} \xi_{y} \\
\xi_{x} & =J^{-1} y_{\eta} \\
\xi_{y} & =-J^{-1} x_{\eta} \\
\eta_{t} & =-x_{\tau} \eta_{x}-y_{\tau} \eta_{y} \\
\eta_{x} & =-J^{-1} y_{\xi} \\
\eta_{y} & =J^{-1} x_{\xi}
\end{aligned}
$$

Substituindo as relações obtidas no termo entre chaves $(\{\cdots\})$ da equação (2.46), obtém-se o seguinte resultado:

$$
\begin{aligned}
& \mathbf{Q}\left[\frac{\partial}{\partial \tau}(J)+\frac{\partial}{\partial \xi}\left(J \xi_{t}\right)+\frac{\partial}{\partial \eta}\left(J \eta_{t}\right)\right]+ \mathbf{E}\left[\frac{\partial}{\partial \xi}\left(J \xi_{x}\right)+\frac{\partial}{\partial \eta}\left(J \eta_{x}\right)\right]+ \\
&+\mathbf{F}\left[\frac{\partial}{\partial \xi}\left(J \xi_{y}\right)+\frac{\partial}{\partial \eta}\left(J \eta_{y}\right)\right]=0
\end{aligned}
$$

Portanto, a equação (2.46) fica simplificada:

$$
\frac{\partial}{\partial \tau}(J \mathbf{Q})+\frac{\partial}{\partial \xi}\left[J\left(\xi_{t} \mathbf{Q}+\xi_{x} \mathbf{E}+\xi_{y} \mathbf{F}\right)\right]+\frac{\partial}{\partial \eta}\left[J\left(\eta_{t} \mathbf{Q}+\eta_{x} \mathbf{E}+\eta_{y} \mathbf{F}\right)\right]=0
$$

e ainda pode ser excrita como:

$$
\frac{\partial \overline{\mathbf{Q}}}{\partial \tau}+\frac{\partial \overline{\mathbf{E}}}{\partial \xi}+\frac{\partial \overline{\mathbf{F}}}{\partial \eta}=0
$$

onde o novo vetor de estados transformado $\overline{\mathbf{Q}}$ pode ser representado da seguinte forma:

$$
\overline{\mathbf{Q}}=J \mathbf{Q} \Rightarrow \overline{\mathbf{Q}}=J\left\{\begin{array}{c}
\rho \\
\rho u \\
\rho v \\
e
\end{array}\right\}
$$

E os vetores de fluxo transformados $\overline{\mathbf{E}}$ e $\overline{\mathbf{F}}$ :

$$
\overline{\mathbf{E}}=J\left(\xi_{t} \mathbf{Q}+\xi_{x} \mathbf{E}+\xi_{y} \mathbf{F}\right)=J\left\{\begin{array}{c}
\xi_{t} \rho+\xi_{x}(\rho u)+\xi_{y}(\rho v) \\
\xi_{t}(\rho u)+\xi_{x}\left(\rho u^{2}+p\right)+\xi_{y}(\rho u v) \\
\xi_{t}(\rho v)+\xi_{x}(\rho u v)+\xi_{y}\left(\rho v^{2}+p\right) \\
\xi_{t} e+\xi_{x}[(e+p) u]+\xi_{y}[(e+p) v]
\end{array}\right\}
$$




$$
\overline{\mathbf{F}}=J\left(\eta_{t} \mathbf{Q}+\eta_{x} \mathbf{E}+\eta_{y} \mathbf{F}\right)=J\left\{\begin{array}{c}
\eta_{t} \rho+\eta_{x}(\rho u)+\eta_{y}(\rho v) \\
\eta_{t}(\rho u)+\eta_{x}\left(\rho u^{2}+p\right)+\eta_{y}(\rho u v) \\
\eta_{t}(\rho v)+\eta_{x}(\rho u v)+\eta_{y}\left(\rho v^{2}+p\right) \\
\eta_{t} e+\eta_{x}[(e+p) u]+\eta_{y}[(e+p) v]
\end{array}\right\}
$$

Definindo as componentes contravariantes de velocidade como:

$$
\begin{aligned}
& U=\xi_{t}^{0}+\xi_{x} u+\xi_{y} v \\
& V=\not \not_{t}^{0}+\eta_{x} u+\eta_{y} v
\end{aligned}
$$

Pode-se escrever os vetores $\overline{\mathbf{E}}$ e $\overline{\mathbf{F}}$ da seguinte maneira:

$$
\overline{\mathbf{E}}=J\left\{\begin{array}{c}
\rho U \\
\rho u U+p \xi_{x} \\
\rho v U+p \xi_{y} \\
(e+p) U
\end{array}\right\} \quad, \quad \overline{\mathbf{F}}=J\left\{\begin{array}{c}
\rho V \\
\rho u V+p \eta_{x} \\
\rho v V+p \eta_{y} \\
(e+p) V
\end{array}\right\}
$$

ou, de outro modo:

$$
\begin{aligned}
\overline{\mathbf{Q}} & \equiv J \mathbf{Q} \\
\overline{\mathbf{E}} & \equiv \mathbf{E}\left(J \frac{\partial \xi}{\partial x}\right)+\mathbf{F}\left(J \frac{\partial \xi}{\partial y}\right) \\
\overline{\mathbf{F}} & \equiv \mathbf{E}\left(J \frac{\partial \eta}{\partial x}\right)+\mathbf{F}\left(J \frac{\partial \eta}{\partial y}\right)
\end{aligned}
$$

Assim, pode-se definir as matrizes Jacobianas $\mathbf{C}_{1}$ e $\mathbf{C}_{2}$ do sistema transformado de forma análoga às matrizes $\mathbf{A}$ e $\mathbf{B}$ do sistema cartesiano:

$$
\begin{aligned}
& \mathbf{C}_{1}=\frac{\partial \overline{\mathbf{E}}}{\partial \mathbf{Q}}=\frac{\partial \mathbf{E}}{\partial \mathbf{Q}}\left(J \frac{\partial \xi}{\partial x}\right)+\frac{\partial \mathbf{F}}{\partial \mathbf{Q}}\left(J \frac{\partial \xi}{\partial y}\right) \\
& \mathbf{C}_{2}=\frac{\partial \overline{\mathbf{F}}}{\partial \mathbf{Q}}=\frac{\partial \mathbf{E}}{\partial \mathbf{Q}}\left(J \frac{\partial \eta}{\partial x}\right)+\frac{\partial \mathbf{F}}{\partial \mathbf{Q}}\left(J \frac{\partial \eta}{\partial y}\right)
\end{aligned}
$$

Com isso, tem-se uma representação das equações de Euler que é válida em qualquer sistema de coordenadas. Esta forma será vital no que se segue, para separar as variações físicas das variações geométricas na derivação das equações adjuntas. 


\subsubsection{Representação Utilizando Notação Indicial}

Como será visto no capítulo seguinte, a derivação do problema adjunto resulta em expressões bastante extensas. De maneira a apresentá-la de uma forma mais clara, é interessante utilizar a notação indicial durante esse processo. Considere, então, as equações de Euler já apresentadas no espaço físico em notação indicial:

$$
\frac{\partial Q_{\alpha}}{\partial t}+\frac{\partial f_{\alpha}^{k^{\prime}}}{\partial x^{k^{\prime}}}=0
$$

onde índices com apóstrofes $\left(x^{k^{\prime}}\right)$ implicam coordenadas cartesianas e os subscritos gregos variam de 1 a 4 (no espaço 2-D), indicando coordenadas no espaço de estado: 1 se refere à eq. da continuidade, 2 e 3 às eqs. de quantidade de movimento e 4 à eq. da energia. O estado $Q_{\alpha}$ e os vetores de fluxos $f_{\alpha}^{k^{\prime}}$ são definidos por:

$$
Q_{\alpha} \Rightarrow\left(\frac{\rho}{\rho u^{i^{\prime}}} \frac{;}{e}\right) \quad f_{\alpha}^{k^{\prime}} \Rightarrow\left(\frac{\rho u^{k^{\prime}}}{\frac{\rho u^{i^{\prime}} u^{k^{\prime}}+p g^{i^{\prime} k^{\prime}}}{(e+p) u^{k^{\prime}}}}\right)
$$

em particular para as equações de conservação de quantidade de movimento, existe uma relação entre o superescrito $i^{\prime}$ e o subscrito $\alpha$ : $i^{\prime}=\alpha-1$ para $2 \leq$ $\alpha \leq 3$. O símbolo $e$ representa a energia total, $e=\rho\left(e_{i}+\mathbf{u} \cdot \mathbf{u} / 2\right), e_{i}$ corresponde à energia interna específica e $g^{i^{\prime} j^{\prime}}$ ao tensor métrico, que se resume a matriz identidade em coordenadas cartesianas.

No espaço transformado (índices sem apóstrofe $\xi^{k}$ ), as equações de Euler em notação indicial são dadas por (REUTHER, 1996):

$$
J \frac{\partial Q_{\alpha}}{\partial t}+J \beta_{i^{\prime}}^{k} \frac{\partial f_{\alpha}^{i^{\prime}}}{\partial \xi^{k}}=0 \Rightarrow \frac{\partial\left(J Q_{\alpha}\right)}{\partial t}+\frac{\partial F_{\alpha}^{k}}{\partial \xi^{k}}=0
$$

onde $\beta_{i^{\prime}}^{k}$ é o operador de transformação de coordenadas entre os espaços físico e transformado. Juntamente com sua inversa, são definidos como:

$$
\beta \Rightarrow \beta_{i^{\prime}}^{k}=\frac{\partial \xi^{k}}{\partial x^{i^{\prime}}} \quad ; \quad \beta^{-1} \Rightarrow \beta_{k}^{i^{\prime}}=\frac{\partial x^{i^{\prime}}}{\partial \xi^{k}} \quad ;
$$

O Jacobiano da transformação é dado por $J=\operatorname{det}\left(\beta_{i^{\prime}}^{k}\right)$, que é assumido independente do tempo. Também se assume que $J$ não se anule nem mude de sinal em nenhum ponto do domínio $\mathcal{D}$.

Além disso, a seguinte identidade tensorial foi utilizada para se obter a última forma:

$$
\frac{\partial\left(J \beta_{i^{\prime}}^{k}\right)}{\partial \xi^{k}}=0 \quad \Rightarrow \quad F_{\alpha}^{k}=J \beta_{i^{\prime}}^{k} f_{\alpha}^{i^{\prime}}
$$


As matrizes generalizadas de fluxo Jacobiano são então definidas com base na eq. (2.67), resultando:

$$
C_{\alpha \beta}^{k}=J \beta_{i^{\prime}}^{k} \frac{\partial f_{\alpha}^{i^{\prime}}}{\partial Q_{\beta}}=J \beta_{i^{\prime}}^{k} A_{\alpha \beta}^{i^{\prime}}
$$

e elas levam a seguinte forma das equações de Euler generalizadas:

$$
\frac{\partial Q_{\alpha}}{\partial t}+\frac{C_{\alpha \beta}^{k}}{J} \frac{\partial Q_{\beta}}{\partial \xi^{k}}=0
$$




\section{TEORia de Controle APLICADA ÀS EquAÇões DO MOVIMENTO}

Este capítulo apresenta a aplicação da abordagem alternativa da formulação contínua do problema adjunto proposta por Cacuci et al. (1980), descrita em linhas gerais na seção 1.4.3, para determinar sensibilidades de uma função objetivo com relação a parâmetros de controle impostos nas fronteiras de entrada de escoamentos modelados pelas equações de Euler 2-D compressíveis em estado estacionário. Nas seções que seguem, encontram-se a derivação das equações adjuntas, suas condições de contorno e das expressões utilizadas para o cálculo do gradiente da medida de mérito para escoamentos nos regimes subsônico e supersônico.

Vale destacar que a independência da formulação contínua com respeito ao método numérico adotado para a simulação do escoamento traz vantagens importantes: permite uma abordagem conceitual mais genérica das equações adjuntas e das suas condições de contorno. Uma rotina assim construída pode, em princípio, ser associada a diferentes códigos de simulação do escoamento.

\subsection{O Problema Variacional}

O principal objetivo de métodos de otimização consiste em minimizar a função que representa uma determinada medida de mérito. Considere, para efeito de derivação das equações adjuntas, aquela apresentada pela eq. (1.2) na seção 1.4 .2 .

As aplicações de interesse aqui tratam principalmente de forças aerodinâmicas em escoamentos estacionários. Assim, o foco recai em funções objetivo que são integradas sobre a superfície aerodinâmica, ao contrário de integrais de domínio - é desnecessário dizer que as primeiras podem ser recuperadas a partir das últimas por meio de distribuições (SCHWARTZ, 1966). Nesse contexto, a forma 
estacionária da eq. (2.65) é imposta em (1.2) e, de acordo com (1.19), resulta:

$$
G=\underbrace{\int_{b_{w}} \mathcal{F}(\mathbf{Q}, \alpha)\left|\frac{\mathrm{d} S^{\prime} \mid}{\mathrm{d} S}\right| \mathrm{d} S}_{I}+\underbrace{\left\langle\phi_{\alpha}, \frac{\partial F_{\alpha}^{k}}{\partial \xi^{k}}\right\rangle}_{I_{c_{1}}}+\underbrace{\langle\psi, \mathcal{B}\rangle_{s}}_{I_{c_{2}}}+\underbrace{\left\langle\mathbf{a}, \mathcal{P}-\mathcal{P}_{o}\right\rangle}_{I_{c_{3}}}
$$

no espaço transformado. Aqui, I representa a medida de mérito original, enquanto $I_{c_{1}}, I_{c_{2}}$ and $I_{c_{3}}$ indicam os funcionais restritivos. Os vetores $\phi_{\alpha}, \psi$ and a são os já mencionados multiplicadores de Lagrange.

Aqui vale acrescentar um comentário a respeito da utilização da forma generalizada das equações Euler estacionárias impostas como restrição ao problema variacional na eq. (3.1). Apesar de não ser essencial para o prosseguimento da derivação, ao mapear uma superfície aerodinâmica em um plano de coordenada constante, essa forma das equações governantes permite que se separe variações físicas e geométricas naturalmente. Em linhas gerais, a idéia é a seguinte: a representação geométrica da superfície sólida é fixa no espaço transformado, ou seja, ela não muda com variações dos parâmetros de controle escolhidos, embora a forma física seja alterada. A única entidade que, na realidade, muda com as variações dos parâmetros $\delta \mathcal{P}$ são os operadores transformação de coordenadas $\beta$, assim como a métrica do espaço transformado. Dessa forma, variações físicas e geométricas podem ser separadas. Isso também simplifica consideravelmente o problema variacional, já que o domínio de integração permanece fixo no espaço transformado.

Agora, da eq. (1.7), a primeira variação de $I$ é explícitamente escrita como:

$$
\delta I=\left\langle\frac{\partial \mathcal{F}}{\partial Q_{\alpha}}\left|\frac{\mathrm{d} S^{\prime}}{\mathrm{d} S}\right|, \delta Q_{\alpha}\right\rangle_{b_{w}}+\left\langle\mathcal{F}, \delta\left|\frac{\mathrm{d} S^{\prime}}{\mathrm{d} S}\right|\right\rangle_{b_{w}}
$$

que identifica a razão de elementos de área entre os espaços físico e transformado, $\left|\mathrm{d} S^{\prime} / \mathrm{d} S\right|$, como único parâmetro de controle que aparece em $I$.

Assim, para os funcionais restritivos, cada um deles será considerado separadamente, abaixo. Primeiro, $I_{c_{1}}$, que envolve a variação das equações governantes, é dado por:

$$
\delta I_{c_{1}}=\left\langle\delta \phi_{\alpha}, \frac{\partial F_{\alpha}^{k}}{\partial \xi^{k}}\right\rangle+\left\langle\phi_{\alpha}, \frac{\partial\left(\delta F_{\alpha}^{k}\right)}{\partial \xi^{k}}\right\rangle
$$

Mas, pela regra da cadeia:

$$
\frac{\partial}{\partial \xi^{k}}\left(J \phi_{\alpha} \delta F_{\alpha}^{k}\right)=J \phi_{\alpha} \frac{\partial\left(\delta F_{\alpha}^{k}\right)}{\partial \xi^{k}}+\delta F_{\alpha}^{k} \frac{\partial\left(J \phi_{\alpha}\right)}{\partial \xi^{k}}
$$


Portanto, substituindo (3.4) em (3.3), tem-se:

$$
\delta I_{c_{1}}=\left\langle\delta \phi_{\alpha}, \frac{\partial F_{\alpha}^{k}}{\partial \xi^{k}}\right\rangle+\int_{\mathcal{D}} \frac{1}{J} \frac{\partial\left(J \phi_{\alpha} \delta F_{\alpha}^{k}\right)}{\partial \xi^{k}} \mathrm{~d} \mathcal{V}-\left\langle\delta F_{\alpha}^{k}, \frac{1}{J} \frac{\partial\left(J \phi_{\alpha}\right)}{\partial \xi^{k}}\right\rangle
$$

Aplicando o teorema de Gauss no segundo termo da expressão (3.5), obtém-se a variação de $I_{c_{1}}$ :

$$
\delta I_{c_{1}}=\left\langle\delta \phi_{\alpha}, \frac{\partial F_{\alpha}^{k}}{\partial \xi^{k}}\right\rangle+\left\langle\phi_{\alpha}, \delta F_{\alpha}^{k} n_{k}\right\rangle_{s}-\left\langle\delta F_{\alpha}^{k}, \frac{1}{J} \frac{\partial\left(J \phi_{\alpha}\right)}{\partial \xi^{k}}\right\rangle
$$

onde a integral de superfície define o concomitante bilinear, $P[\phi, \delta \mathbf{Q}]_{s}$, e $n_{k}$ é o versor normal apontando para dentro do domínio $\mathcal{D}$. O subscrito $s$ se refere a toda fronteira, $\partial \mathcal{D}$, que inclui a superfície do corpo $\left(b_{w}\right)$, o farfield $\left(b_{\infty}\right)$ e os planos de corte $\left(b_{c p}\right)$. As variações de $I_{c_{2}}$ e $I_{c_{3}}$ seguem diretamente das diferenciais de Fréchet, como na eq. (1.24). Agora, o objetivo deste trabalho é, particularmente, controlar as condições de contorno em fronteiras de entrada de escoamento. Portanto,

$$
\delta I_{c_{2}}+\delta I_{c_{3}}=\langle\delta \psi, \mathcal{B}\rangle_{s_{i}}+\left\langle\psi, \mathcal{B}_{Q}^{\prime} \delta \mathbf{Q}\right\rangle_{s_{i}}+\left\langle\psi, \mathcal{B}_{\mathcal{P}}^{\prime} \delta \mathcal{P}\right\rangle_{s_{i}}+\left\langle\delta \mathbf{a}, \mathcal{P}-\mathcal{P}_{o}\right\rangle+\langle\mathbf{a}, \delta \mathcal{P}\rangle
$$

onde a notação \langle\rangle$_{s_{i}}$ naturalmente se refere à fronteira de entrada, que é a porção de $b_{\infty}$ onde $\mathbf{u} \cdot \mathbf{n}>0$.

A variação do funcional aumentado (3.1) é então determinada ao subtrair as eqs. (3.6) e (3.7) da eq. (3.2). Separando as integrais de superfície que constroem o concomitante bilinear de acordo com cada fronteira, tem-se:

$$
\begin{aligned}
\delta G & =\left\langle\delta \phi_{\alpha}, \frac{\partial F_{\alpha}^{k}}{\partial \xi^{k}}\right\rangle+\langle\delta \psi, \mathcal{B}\rangle_{s_{i}}+\left\langle\delta \mathbf{a}, \mathcal{P}-\mathcal{P}_{o}\right\rangle-\left\langle\delta F_{\alpha}^{k}, \frac{1}{J} \frac{\partial\left(J \phi_{\alpha}\right)}{\partial \xi^{k}}\right\rangle+ \\
& +\left\langle\frac{\partial \mathcal{F}}{\partial Q_{\alpha}}\left|\frac{\mathrm{d} S^{\prime}}{\mathrm{d} S}\right|, \delta Q_{\alpha}\right\rangle_{b_{w}}+\left\langle\psi, \mathcal{B}_{Q}^{\prime} \delta \mathbf{Q}\right\rangle_{s_{i}}+\left\{\left\langle\phi_{\alpha}, \delta F_{\alpha}^{k} n_{k}\right\rangle_{b_{w}}+\right. \\
& \left.+\left\langle\phi_{\alpha}, \delta F_{\alpha}^{k} n_{k}\right\rangle_{s_{i}}+\left[\left\langle\phi_{\alpha}, \delta F_{\alpha}^{k} n_{k}\right\rangle_{b_{c p}}\right]_{b_{c p}^{+}}^{b_{c p}^{-}}+\left\langle\phi_{\alpha}, \delta F_{\alpha}^{k} n_{k}\right\rangle_{s_{o}}\right\}+ \\
& +\left\langle\mathcal{F}, \delta\left|\frac{\mathrm{d} S^{\prime}}{\mathrm{d} S}\right|\right\rangle_{b_{w}}+\langle\mathbf{a}, \delta \alpha\rangle+\left\langle\psi, \mathcal{B}_{\mathcal{P}}^{\prime} \delta \mathcal{P}\right\rangle_{s_{i}}
\end{aligned}
$$

onde a notação \langle\rangle$_{s_{o}}$ se refere a fronteira de saída, i.e., a parte de $b_{\infty}$ em que $\mathbf{u} \cdot \mathbf{n} \leq 0$, e \langle\rangle$_{b_{c p}}$ se refere aos planos de corte. Os colchetes implicam que o termo dentro deles seja integrado sobre cada lado do plano de corte, com vetores normais opostos. Daqui, simplesmente impondo condições de contorno periódicas em $\phi_{\alpha}$ dadas pela eq. (3.9), as integrais são automaticamente canceladas (HAYASHI; CEZE; VOLPE, 2013).

$$
\left.\phi_{\alpha}\right|_{b_{c p}^{+}}=\left.\phi_{\alpha}\right|_{b_{c p}^{-}}
$$


Note, no entanto, que a imposição desse tipo de condição de contorno requer que as variáveis adjuntas $\phi_{\alpha}$ e todas as suas derivadas sejam contínuas através do plano de corte $b_{c p}$.

Outra condição de realizabilidade física implica que a velocidade normal em uma superfície sólida $\left(b_{w}\right)$ seja zero, $\mathbf{u} \cdot \mathbf{n}=0$. Esse requisito restringe o vetor de fluxo normal à $\left(b_{w}\right)$ :

$$
\left.F_{\alpha}^{k}\right|_{\xi^{2}=0}=J^{-1}\left\{\begin{array}{c}
0 \\
p \xi_{x}^{2} \\
p \xi_{y}^{2} \\
0
\end{array}\right\}
$$

e, naturalmente, sua primeira variação (HAYASHI; CEZE; VOLPE, 2013; REUTHER, 1996; JAMESON; KIM, 2003b), como mostra a eq. (3.11) abaixo:

$$
\left.\delta F_{\alpha}^{k}\right|_{\xi^{2}=0}=J\left\{\begin{array}{c}
0 \\
\delta p \xi_{x}^{2} \\
\delta p \xi_{y}^{2} \\
0
\end{array}\right\}+p\left\{\begin{array}{c}
0 \\
\delta\left(J \xi_{x}^{2}\right) \\
\delta\left(J \xi_{y}^{2}\right) \\
0
\end{array}\right\}
$$

Ou seja, os componentes de massa e energia desaparecem identicamente. Assumindo que a parede seja mapeada por um plano coordenado $\xi^{2}, b_{w} \Rightarrow \xi^{2}=0$, e usando a relação $\alpha=i^{\prime}+1$, a equação de conservação da quantidade de movimento (2.64), se reduz à:

$$
\left.\phi_{\alpha} \delta F_{\alpha}^{k} n_{k}\right|_{b_{w}}=\left.\phi_{\alpha} \delta F_{\alpha}^{k} n_{k}\right|_{\xi^{2}=0}=\delta p\left[\phi_{\left(i^{\prime}+1\right)} J \beta_{i^{\prime}}^{2} n_{2}\right]+p\left[\phi_{\left(i^{\prime}+1\right)} \delta\left(J \beta_{i^{\prime}}^{2}\right) n_{2}\right]
$$

Por fim, a variação dos vetores de fluxo para o restante do domínio computacional é obtida ao combinar as eqs. (2.67) e (2.68):

$$
\delta F_{\alpha}^{k}=C_{\alpha \beta}^{k} \delta Q_{\beta}+\delta\left(J \beta_{i^{\prime}}^{k}\right) f_{\alpha}^{i^{\prime}}
$$

onde o primeiro termo representa a parte física, enquanto o segundo, a parte paramétrica da variação total.

Ao substituir as eqs. (3.12) e (3.13) para os termos correspondentes na eq. (3.8), impor a periodicidade em $\phi_{\alpha}$ nos planos de corte, e rearranjar termos de maneira apropriada, obtém-se a seguinte expressão para $\delta G$ : 


$$
\begin{aligned}
& \delta G=\underbrace{\left\langle\delta \phi_{\alpha}, \frac{\partial F_{\alpha}^{k}}{\partial \xi^{k}}\right\rangle+\langle\delta \psi, \mathcal{B}\rangle_{s_{i}}+\left\langle\delta \mathbf{a}, \mathcal{P}-\mathcal{P}_{o}\right\rangle}_{(a)}-\underbrace{\left\langle\frac{C_{\alpha \beta}^{k}}{J} \frac{\partial\left(J \phi_{\alpha}\right)}{\partial \xi^{k}}, \delta Q_{\beta}\right\rangle}_{(b)}+ \\
& +\underbrace{\left\langle\psi_{\alpha} \frac{\partial \mathcal{B}_{\alpha}}{\partial Q_{\beta}}+\phi_{\alpha} C_{\alpha \beta}^{2} n_{2}, \delta Q_{\beta}\right\rangle_{s_{i}}}_{(c)}+\underbrace{\left\langle\phi_{\alpha} C_{\alpha \beta}^{2} n_{2}, \delta Q_{\beta}\right\rangle_{s_{o}}}_{(d)}+\underbrace{\left\langle\phi_{\alpha}, \delta\left(J \beta_{i^{\prime}}^{2}\right) f_{\alpha}^{i^{\prime}} n_{2}\right\rangle_{b_{\infty}}}_{(e)}+ \\
& +\underbrace{\left\langle\frac{\partial \mathcal{F}}{\partial Q_{\alpha}}\left|\frac{\mathrm{d} S^{\prime}}{\mathrm{d} S}\right|+\left[\phi_{\left(i^{\prime}+1\right)} J \beta_{i^{\prime}}^{2} n_{2}\right] \frac{\partial p}{\partial Q_{\alpha}}, \delta Q_{\alpha}\right\rangle_{b_{w}}}_{(f)}-\underbrace{\left\langle\delta\left(J \beta_{i^{\prime}}^{k}\right), \frac{f_{\alpha}^{i^{\prime}}}{J} \frac{\partial\left(J \phi_{\alpha}\right)}{\partial \xi^{k}}\right\rangle}_{(g)}+ \\
& +\underbrace{\left\langle\mathcal{F}, \delta\left|\frac{\mathrm{d} S^{\prime}}{\mathrm{d} S}\right|\right\rangle_{b_{w}}+\left\langle p,\left[\phi_{\left(i^{\prime}+1\right)} \delta\left(J \beta_{i^{\prime}}^{2}\right) n_{2}\right]\right\rangle_{b_{w}}}_{(h)}+\underbrace{\langle\mathbf{a}, \delta \mathcal{P}\rangle+\left\langle\psi, \mathcal{B}_{\mathcal{P}}^{\prime} \delta \mathcal{P}\right\rangle_{s_{i}}}_{(i)}
\end{aligned}
$$

onde as variações paramétricas dos fluxos nas seções de entrada e saída das fronteiras de farfield foram agrupadas no último termo da segunda linha, na forma $s_{i} \cup s_{o} \Rightarrow b_{\infty}$. Também foi assumido que o que essa fronteira é mapeada em um plano de coordenada constante, $b_{\infty} \Rightarrow \xi^{2}=1$. Uma separação entre as variações física e paramétrica é aparente na equação acima. Os termos $a, b, c, d$ e $f$ pertencem ao primeiro grupo, que dá origem ao problema adjunto. Os termos restantes $e, g, h$ e $i$ são parte do gradiente de sensibilidade. Os termos $e, g$ e $h$ ainda podem ser simplificados como mostra o apêndice B (JAMESON; KIM, 2003b).

\subsection{As Equações Adjuntas}

A equação adjunta é obtida a partir do termo b, na eq. (3.14), e segue o mesmo procedimento já amplamente discutido na literatura (HAYASHI; CEZE; VOLPE, 2013; REUTHER, 1996; JAMESON; KIM, 2003b). Basta encontrar um vetor $\phi_{\alpha}$ não-trivial que leve o produto interno a zero para qualquer variação arbitrária, porém realizável, $\delta \mathbf{Q}$. O procedimento dá origem a forma estacionária da equação adjunta.

Entretanto, a solução adjunta estacionária é usualmente computada de forma análoga ao que se faz com o escoamento, ou seja, postulando um termo tempodependente para então realizar uma marcha no tempo até que se atinja uma solução estacionária (REUTHER, 1996). Dessa forma, tem-se:

$$
\frac{\partial \phi_{\alpha}}{\partial t}-\frac{C_{\beta \alpha}^{k}}{J} \frac{\partial\left(J \phi_{\beta}\right)}{\partial \xi^{k}}=0
$$


Ao comparar as equação de Euler original (2.69) com a eq. (3.15) acima, pode-se notar que a matriz de fluxo Jacobiano é transposta e tem seu sinal trocado com relação à primeira. Assim, o caráter hiperbólico das equações de Euler é mantido no problema dual. Por simplicidade, a variável adjunta pode ser redefinida de maneira que leve em consideração a transformação Jacobiana, $\varphi_{\alpha} \equiv J \phi_{\alpha}$, que leva a expressão:

$$
\frac{\partial \varphi_{\alpha}}{\partial t}-C_{\beta \alpha}^{k} \frac{\partial \varphi_{\beta}}{\partial \xi^{k}}=0
$$

Apesar deste trabalho ter foco apenas em soluções de estado estacionário, a inclusão do termo tempo-dependente para a resolução das equações adjuntas é crucial no que tange a formulação das condições de contorno propostas por Hayashi, Ceze e Volpe (2013), que fazem uso do caráter hiperbólico de ambos os problemas primal e dual, com problemas de Riemann complementares.

\subsection{Condições de Contorno Adjuntas}

Claramente, o problema de contorno adjunto é formulado com base nos termos $c, d$ e $f$ da eq. (3.14), já que são os termos que envolvem integrais nas fronteiras do domínio que incluem variações físicas $\delta \mathbf{Q}$. Esses produtos internos devem ser levados a zero, de uma forma que sejam consistentes com as condições de contorno do problema físico e que façam da equação adjunta bem-posta. Esse assunto foi largamente discutido por Hayashi, Ceze e Volpe (2013), de tal forma que apenas os resultados aqui relevantes serão relatados.

De forma a colocá-los numa ordem crescente de complexidade, os planos de corte são, certamente, as condições de contorno mais simples. Tudo que ela requer é que haja periodicidade das variáveis adjuntas ao cruzar esses planos, em uma conexão com a eq. (3.8).

Para paredes sólidas $\left(b_{w}\right)$, a restrição de realizabilidade é dada pela eq. (3.12). Sua introdução na expressão para $\delta G$ (3.14) dá origem ao termo $f$, que também envolve o funcional objetivo através da derivada $\partial \mathcal{F} / \partial Q_{\beta}$. A necessidade de conduzir esse termo a zero para uma variação $\delta \mathbf{Q}$ arbitrária leva a simples condição de contorno de parede, que pode ser escrita na forma:

$$
\varphi_{\left(i^{\prime}+1\right)} J \beta_{i^{\prime}}^{2} n_{2}=\left.\varphi_{\left(i^{\prime}+1\right)} n_{i^{\prime}}\right|_{b_{w}}=-\frac{\partial \mathcal{F}}{\partial Q_{\beta}} \frac{\partial Q_{\beta}}{\partial p}\left|\frac{\mathrm{d} S^{\prime}}{\mathrm{d} S}\right|=-\frac{\partial \mathcal{F}}{\partial p}\left|\frac{\mathrm{d} S^{\prime}}{\mathrm{d} S}\right|
$$

Esta condição é acoplada com o esquema de Reuther (1996) ao extrapolar as 
variáveis adjuntas em paredes sólidas.

As condições de contorno de farfield são obtidas a partir dos termos $c$ e $d$, que podem ser postos na forma:

$$
\Delta_{c_{1}}+\Delta_{c_{2}}+\Delta_{d}=\underbrace{\left\langle\beta_{\alpha} \frac{\partial B_{\alpha}}{\partial Q_{\beta}}, \delta Q_{\beta}\right\rangle_{s_{i}}+\left\langle\varphi_{\alpha} A_{\alpha \beta}^{i^{\prime}} n_{i^{\prime}}, \delta Q_{\beta}\right\rangle_{s_{i}}}_{(c)}+\underbrace{\left\langle\varphi_{\alpha} A_{\alpha \beta}^{i^{\prime}} n_{i^{\prime}}, \delta Q_{\beta}\right\rangle_{s_{o}}}_{(d)}
$$

onde foi feito uso da eq. (2.68) e $c$ foi dividido em 2 termos, de forma a associá-los com suas contrapartes na eq. (1.24). O primeiro termo no lado direito da equação, aqui, corresponde ao quinto em (1.24). Enquanto o segundo e terceiro termos são ambos concomitantes bilineares, um para a seção de entrada $\left(s_{i}\right)$ e outro para a seção de saída $\left(s_{o}\right)$ das fronteiras permeáveis. Por conveniência futura, eles são nomeados $\Delta_{c_{1}}, \Delta_{c_{2}}$ e $\Delta_{d}$, respectivamente. Os concomitantes bilineares $\Delta_{c_{2}}$ e $\Delta_{d}$ devem ser decompostos de acordo com a eq. (1.24).

Antes de dar prosseguimento, deve-se notar que a equação adjunta (3.16) é generalizada. Ela vale para qualquer sistema de coordenadas e, particularmente, para o cartesiano. O mesmo se aplica às condições de contorno que são impostas nos planos de corte, paredes sólidas e farfield. No primeiro caso, os requisitos de periodicidade permanecem imutáveis, enquanto para os outros dois, eqs. (3.17) e (3.18) apresentam suas formas cartesianas. Assim, prosseguir-se-á com o mesmo que foi discutido por Hayashi, Ceze e Volpe (2013), e resolver o problema adjunto diretamente no espaço físico, que é representado pelas coordenadas cartesianas.

\subsubsection{O Problema 2-D}

Para escoamentos bidimensionais, os subscritos Gregos variam de 1 a 4, onde 1 se refere a continuidade, 2 e 3 para quantidade de movimento e 4 para a energia. Índices latinos se referem às duas dimensões no espaço físico - por simplicidade, $x^{1^{\prime}}=x, x^{2^{\prime}}=y$. As matrizes de fluxo Jacobiano correspondentes são desginadas por: $\mathbf{A}^{1^{\prime}}=\mathbf{A}$ e $\mathbf{A}^{2^{\prime}}=\mathbf{B}$, respectivamente. Sob estas circunstâncias, a equação adjunta pode ser escrita na forma:

$$
\frac{\partial \varphi_{\alpha}}{\partial t}-A_{\beta \alpha} \frac{\varphi_{\beta}}{\partial x}-B_{\beta \alpha} \frac{\varphi_{\beta}}{\partial y}=0
$$

O operador fluxo Jacobiano é simbolizado por $A_{\beta \alpha}^{i^{\prime}} \Rightarrow \overline{\mathbf{A}}^{T}=\left(\mathbf{A}^{T}, \mathbf{B}^{T}\right)$, por simplicidade (HIRSCH, 1988b). Sua projeção na direção normal a uma dada fron- 
teira, $\mathbf{k}=\left(k_{x}, k_{y}\right)^{T}$, é definida da seguinte forma:

$$
-\mathbb{A}_{K}^{T} \equiv-\overline{\mathbf{A}}^{T} \cdot \mathbf{k}=-k_{x} \mathbf{A}^{T}-k_{y} \mathbf{B}^{T}
$$

onde o sinal negativo e a transposição, da eq. (3.19), foi mantida por conveniência. A matriz de transformação de similaridade que diagonaliza a matriz resultante fornece:

$$
\begin{aligned}
-\boldsymbol{\Lambda} & =-\mathbf{P}^{T} \cdot \mathbb{A}_{K}^{T} \cdot\left(\mathbf{P}^{-1}\right)^{T} \\
& =\left(\begin{array}{cccc}
-\mathbf{u} \cdot \mathbf{n} & 0 & 0 & 0 \\
0 & -\mathbf{u} \cdot \mathbf{n} & 0 & 0 \\
0 & 0 & -(\mathbf{u} \cdot \mathbf{n}+c) & 0 \\
0 & 0 & 0 & -(\mathbf{u} \cdot \mathbf{n}-c)
\end{array}\right)
\end{aligned}
$$

que são, obviamente, os mesmos autovalores das equações de Euler, mas com sinais opostos. Também associados com a direção k, os operadores de transformação de similaridade são dados por (HIRSCH, 1988b):

$$
\begin{aligned}
\mathbf{P}^{T}= & \left(\begin{array}{cccc}
1 & u & v & \frac{\mathbf{u} \cdot \mathbf{u}}{2} \\
0 & \rho k_{y} & -\rho k_{x} & \rho\left(u k_{y}-v k_{x}\right) \\
\frac{\rho}{2 c} & \frac{\rho\left(c k_{x}+u\right)}{2 c} & \frac{\rho\left(c k_{y}+v\right)}{2 c} & \frac{\rho}{2}\left(\frac{c}{\gamma-1}+\mathbf{u} \cdot \mathbf{n}+\frac{\mathbf{u} \cdot \mathbf{u}}{2 c}\right) \\
\frac{\rho}{2 c} & \frac{\rho\left(v-c k_{x}\right)}{2 c} & \frac{\rho\left(v-c k_{y}\right)}{2 c} & \frac{\rho}{2}\left(\frac{c}{\gamma-1}-\mathbf{u} \cdot \mathbf{n}+\frac{\mathbf{u} \cdot \mathbf{u}}{2 c}\right)
\end{array}\right) \\
\left(\mathbf{P}^{-1}\right)^{T}= & \left(\begin{array}{cccc}
1-\frac{(\gamma-1)(\mathbf{u} \cdot \mathbf{u})}{2 c^{2}} & \frac{v k_{x}-u k_{y}}{\rho} & \frac{(\gamma-1)(\mathbf{u} \cdot \mathbf{u})-2 c(\mathbf{u} \cdot \mathbf{n})}{2 \rho c} & \frac{(\gamma-1)(\mathbf{u} \cdot \mathbf{u})+2 c(\mathbf{u} \cdot \mathbf{n})}{2 \rho c} \\
\frac{(\gamma-1) u}{c^{2}} & \frac{k_{y}}{\rho} & \frac{c k_{x}-(\gamma-1) u}{\rho c} & \frac{-c k_{x}-(\gamma-1) u}{\rho c} \\
\frac{(\gamma-1) v}{c^{2}} & -\frac{k_{x}}{\rho} & \frac{c k_{y}-(\gamma-1) v}{\rho c} & \frac{-c k_{y}-(\gamma-1) v}{\rho c} \\
-\frac{(\gamma-1)}{c^{2}} & 0 & \frac{\gamma-1}{\rho c} & \frac{\gamma-1}{\rho c}
\end{array}\right)
\end{aligned}
$$

A forma característica da equação adjunta é obtida ao pré-multiplicar (3.19) por $\mathbf{P}^{T}$, que leva a expressão:

$$
\mathbf{P}^{T} \frac{\partial \boldsymbol{\varphi}}{\partial t}-\mathbf{P}^{T} \mathbf{A}^{T}\left(\mathbf{P}^{-1}\right)^{T} \mathbf{P}^{T} \frac{\partial \boldsymbol{\varphi}}{\partial x}-\mathbf{P}^{T} \mathbf{B}^{T}\left(\mathbf{P}^{-1}\right)^{T} \mathbf{P}^{T} \frac{\partial \boldsymbol{\varphi}}{\partial y}=0
$$

Pode ser acresecentado que o produto $\left(\mathbf{P}^{T} \delta \boldsymbol{\varphi}\right)$ define os diferenciais adjuntos de Riemann. Consequentemente, ao computar os produtos de matrizes na eq. (3.24), pode-se obter um sistema de equações de Riemann, ou características, na forma:

$$
\sum_{\beta=1}^{N} K_{\alpha \beta}^{t} \frac{\partial \varphi_{\beta}}{\partial t}=\sum_{\beta=1}^{N} K_{\alpha \beta}^{x} \frac{\partial \varphi_{\beta}}{\partial x}+\sum_{\beta=1}^{N} K_{\alpha \beta}^{y} \frac{\partial \varphi_{\beta}}{\partial y}
$$

onde $N=4$ e os coeficientes $K_{\alpha \beta}^{t}, K_{\alpha \beta}^{x}$ e $K_{\alpha \beta}^{y}$ estão listados na tabela A.1 apresen- 
tada no apêndice A. O conjunto acima mantém a linearidade da equação original (3.19). A única diferença reside no que cada equação $\alpha$ de (3.25) está associada com uma particular velocidade característica, ou seja, o autovalor correspondente da eq. (3.21).

Ao tomar o vetor $\mathbf{k}$ normal às fronteiras do domínio, esses autovalores determinam qual das características adjuntas entram ou deixam o domínio. Como discutido por Hayashi, Ceze e Volpe (2013), as características que entram no domínio são substituídas por condições de contorno, que são obtidas ao encontrar uma relação de compatibilidade entre as variáveis adjuntas $\varphi_{\alpha}$ que anule o concomitante bilinear da fronteira em questão para qualquer variação arbitrária, porém realizável, de $\delta Q_{\alpha}$. Já as características que saem do domínio fornecem para a fronteira a informação vinda do escoamento, completando assim o problema de Riemann. As equações que representam as últimas são tomadas a partir da eq. (3.25) para cada caso.

\subsubsection{Parede Sólida}

Em uma transformação 2-D do tipo $(x, y) \rightleftharpoons(\xi, \eta)$, o contorno do corpo pode ser especificado tanto como uma curva de nível, como $\eta(x, y)=0$, ou em forma paramétrica por $x(\xi, 0)$ and $y(\xi, 0)$. Em qualquer das duas, a razão dos elementos de área pode ser escrita como:

$$
\left|\frac{\mathrm{d} S^{\prime}}{\mathrm{d} S}\right|=\sqrt{\left(\frac{\partial x}{\partial \xi}\right)^{2}+\left(\frac{\partial y}{\partial \xi}\right)^{2}}=\sqrt{g_{11}}
$$

onde $g_{11}$ é um elemento do tensor métrico $g_{i j}$ (FLÜGGE, 1972). Assim, a condição de contorno de parede sólida da eq. (3.17) se torna:

$$
k_{x} \varphi_{2}+k_{y} \varphi_{3}=\frac{\partial \mathcal{F}}{\partial p} \sqrt{g_{11}}
$$

Em um esquema numérico onde o valor das variáveis adjuntas nas faces de um elemento é aproximada pela média aritmética de $\varphi_{\alpha}$ dos elementos adjacentes a ela (HAYASHI, 2009), a condição de contorno adjunta discretizada em paredes sólidas é dada por:

$$
k_{x} \frac{\left(\varphi_{2}^{-}+\varphi_{2}^{+}\right)}{2}+k_{y} \frac{\left(\varphi_{3}^{-}+\varphi_{3}^{+}\right)}{2}=\frac{\partial \mathcal{F}}{\partial p} \sqrt{g_{11}}
$$

onde $\varphi_{k}^{-}$representam os valores das variáveis adjuntas nos volumes fantasmas (ghost cells), enquanto $\varphi_{k}^{+}$correspondem aos valores nos elementos internos correspondentes. 
O fato desta condição possuir duas variáveis adjuntas a serem determinadas nos volumes fantasmas e apenas uma equação restritiva, naturalmente implica em infinitas combinações de $\varphi_{2}^{-}$e $\varphi_{3}^{-}$que satisfazem a eq. (3.28). Nesta Tese, optou-se pelo já mencionado esquema de Reuther (1996), que se adequa bem ao escopo deste trabalho.

$$
\left\{\begin{array}{l}
\varphi_{1}^{-}=\varphi_{1}^{+} \\
\varphi_{2}^{-}=\varphi_{2}^{+}+\frac{2 k_{x}}{k_{x}^{2}+k_{y}^{2}}\left(\frac{\partial \mathcal{F}}{\partial p} \sqrt{g_{11}}-k_{x} \varphi_{2}^{+}-k_{y} \varphi_{3}^{+}\right) \\
\varphi_{3}^{-}=\varphi_{3}^{+}+\frac{2 k_{y}}{k_{x}^{2}+k_{y}^{2}}\left(\frac{\partial \mathcal{F}}{\partial p} \sqrt{g_{11}}-k_{x} \varphi_{2}^{+}-k_{y} \varphi_{3}^{+}\right) \\
\varphi_{4}^{-}=\varphi_{4}^{+}
\end{array}\right.
$$

Esse esquema tem sido amplamente verificado na literatura. Foi mostrado que ele leva a estimativas do gradiente de sensibilidade que estão de acordo com aqueles obtidos por outros métodos, dentro de níveis de precisão razoáveis (REUTHER, 1996). Assim, ele deve ser consistente com as aplicações propostas.

\subsubsection{Saída}

Como já referido, a imposição de condições de contorno em uma determinada fronteira do domínio computacional está diretamente relacionada com o sentido de propagação de características. A figura 3.1 apresenta o sentido de propagação de cada uma delas em fronteiras permeáveis onde ocorre a saída de escoamento.

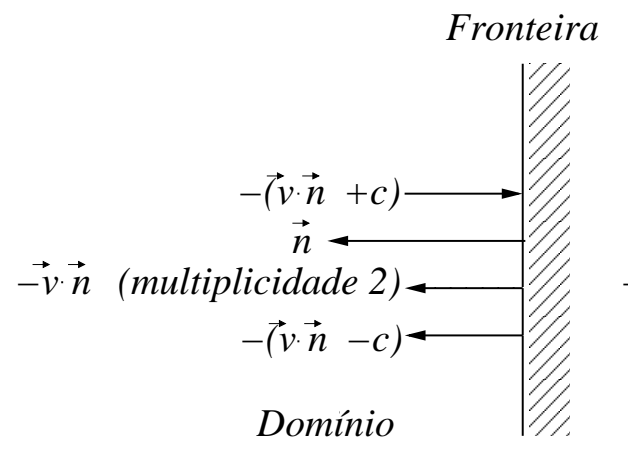

(a) Caso subsônico

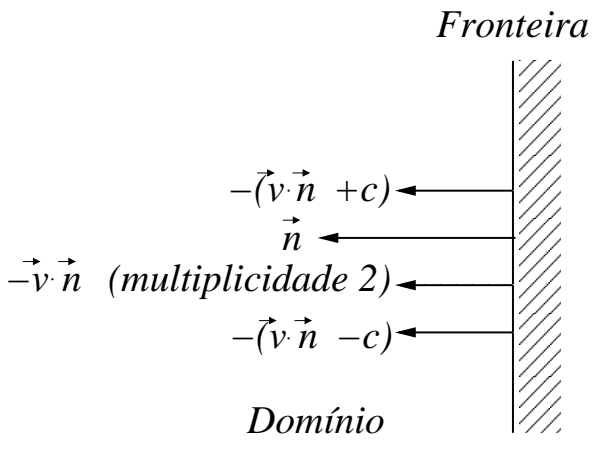

(b) Caso supersônico

Figura 3.1: Propagação de características adjuntas em fronteiras de saída

As expressões para essa condição de contorno são obtidas a partir do termo $d$ nas equações (3.14) e (3.18). Como esse tipo de fronteira em particular não está sujeito a qualquer controle paramétrico, a condição de contorno a ser imposta resulta diretamente da anulação do concomitante bilinear $\Delta_{d}$, reapresentado na eq. (3.30), para variações arbitrárias, porém realizáveis das propriedades do es- 
coamento.

$$
\Delta_{d}=\left\langle\varphi_{\alpha} A_{\alpha \beta}^{i^{\prime}} n_{i^{\prime}}, \delta Q_{\beta}\right\rangle_{s_{o}} \Leftrightarrow\left\langle\boldsymbol{\varphi}^{T} \cdot \mathbb{A}_{K} \cdot \delta \mathbf{Q}\right\rangle_{s_{o}}=0
$$

A derivação segue o mesmo procedimento proposto por Hayashi, Ceze e Volpe (2013), de modo que permaneça consistente com o fato de que as equações governantes e adjuntas sejam bem postas.

O problema de contorno é um tanto trivial para saídas supersônicas. Uma vez que elas não requerem qualquer condição de contorno física e, portanto, qualquer variação $\delta \mathbf{Q}$ é possível, a única maneira de garantir que $\Delta_{d}$ seja nulo é aplicar condições de contorno adjuntas homogêneas $\left.\varphi_{\alpha}\right|_{s_{o}}=0$ :

Por outro lado, absolutamente diferentes, seções de saída em regime de escoamento subsônico implicam a presença de três características adjuntas entrando no domínio. Elas representam o primeiro, segundo e quarto autovalores em (3.21) e suas linhas correspondentes em (3.25) devem ser substituídas por condições de contorno. Assim, apenas a terceira característica adjunta, que é associada ao autovalor $-(\mathbf{u} \cdot \mathbf{n}+c)$, precisa ser resolvida, na realidade.

Portanto, as fronteiras de saída subsônica requerem três condições de contorno adjuntas. Elas vem da única restrição de realizabilidade que é imposta em $\delta Q_{\alpha}$ nessa fronteira. Nas aplicações de interesse deste trabalho, a pressão estática é a única quantidade que é fixada. Ao escrevê-la em função das variáveis conservadas Q e impor que sua variação $\delta p$ seja nula, obtém-se a seguinte relação (HAYASHI; CEZE; VOLPE, 2013):

$$
\delta Q_{4}=u \delta Q_{2}+v \delta Q_{3}-\frac{\left(u^{2}+v^{2}\right)}{2} \delta Q_{1}
$$

A equação acima representa o locus de variações realizáveis nas fronteiras de saída subsônica, i.e., aquelas que correspondem à imposição da condição de contorno: pressão estática constante. Ela preserva três graus de liberdade físicos, uma vez que fornece um $\delta Q_{4}$ realizável em termos de $\delta Q_{1}, \delta Q_{2}$ e $\delta Q_{3}$, que podem ser arbitrários. Em essência, os graus de liberdade físicos preservados correspondem a características do escoamento que saem do domínio e, então, representam uma solução do escoamento e suas variações.

Substituindo (3.31) para a variação correspondente no concomitante bilinear dado pela eq. (3.30), e forçando-o a zero, obtém-se três condições de contorno 
adjuntas correspondentes (HAYASHI; CEZE; VOLPE, 2013):

$$
\left\{\begin{aligned}
\varphi_{1} & =\frac{1}{2}\left[2 e_{t} \gamma-(\gamma-1)\left(u^{2}+v^{2}\right)\right] \varphi_{4}=C_{1} \varphi_{4} \\
\varphi_{2} & =\left[\frac{-2 e_{t} \gamma k_{x}+(\gamma-2) k_{x} u^{2}-2 k_{y} u v+\gamma k_{x} v^{2}}{2\left(k_{x} u+k_{y} v\right)}\right] \varphi_{4}=C_{2} \varphi_{4} \\
\varphi_{3} & =\left[\frac{-2 e_{t} \gamma k_{y}+(\gamma-2) k_{y} v^{2}-2 k_{x} u v+\gamma k_{y} u^{2}}{2\left(k_{x} u+k_{y} v\right)}\right] \varphi_{4}=C_{3} \varphi_{4}
\end{aligned}\right.
$$

A tabela 3.1 apresenta um resumo das condições de contorno de saída em ambos os regimes de escoamento subsônico e supersônico.

Tabela 3.1: Resumo das condições de contorno adjuntas de saída

\begin{tabular}{ccc}
\hline \hline Regime & Variação $\delta \mathbf{Q}$ & Condição adjunta $\boldsymbol{\varphi}$ \\
\hline \multirow{2}{*}{ subsônico } & $\delta Q_{4}\left(\delta Q_{1}, \delta Q_{2}, \delta Q_{3}\right)$ & $\varphi_{1}\left(\varphi_{4}\right), \varphi_{2}\left(\varphi_{4}\right), \varphi_{3}\left(\varphi_{4}\right)$ \\
& eq. $(3.31)$ & eq. $(3.32)$ \\
\hline supersônico & $\delta Q_{\alpha}$ livres & $\varphi_{\alpha}=0$ \\
\hline \hline
\end{tabular}

Em termos de operadores, as eqs. (3.31) e (3.32) correspondem a $\mathcal{B}_{Q}^{\prime} \cdot \delta \mathbf{Q}=0$ e $\mathcal{B}^{*} \cdot \boldsymbol{\varphi}=0$, respectivamente. A primeira vem da eq. (1.20b) e leva em conta o fato de que $\mathcal{B}_{\mathcal{P}}^{\prime}=0$ na saída. As expressões para $\mathcal{B}_{Q}^{\prime}$ e $\mathcal{B}^{*}$ são dadas por:

$$
\left.\mathcal{B}_{Q}^{\prime}\right|_{s_{o}}=\left(\begin{array}{cccc}
0 & 0 & 0 & 0 \\
0 & 0 & 0 & 0 \\
0 & 0 & 0 & 0 \\
-\frac{\mathbf{u} \cdot \mathbf{u}}{2} & u & v & -1
\end{array}\right) \quad ;\left.\quad \mathcal{B}^{*}\right|_{s_{o}}=\left(\begin{array}{cccc}
1 & 0 & 0 & -C_{1} \\
0 & 1 & 0 & -C_{2} \\
0 & 0 & 1 & -C_{3} \\
0 & 0 & 0 & 0
\end{array}\right)
$$

As três primeiras linhas de $\mathcal{B}_{Q}^{\prime}$ se referem aos graus de liberdade livres $\left(\delta Q_{1}\right.$, $\left.\delta Q_{2}, \delta Q_{3}\right)$, que, por sua vez, correspondem às três características que deixam o domínio no problema físico. Enquanto a última linha de $\mathcal{B}^{*}$ implica que $\varphi_{4}$ seja obtido ao resolver a única característica adjunta que sai do domínio, ou seja, a terceira.

Sob essas condições, a decomposição proposta do concomitante bilinear na eq. (3.30) é dada por:

$$
\left\{\begin{array}{l}
P_{1}(\boldsymbol{\varphi})=-\boldsymbol{\varphi}^{T} \mathbb{A}_{K} \\
\mathcal{B}^{*}(\boldsymbol{\varphi})=\boldsymbol{\varphi}^{T} \mathcal{B}^{* T}
\end{array}\right.
$$

Então, levando em conta o fato de que $\Delta_{d}$ é, na realidade, um escalar e ao substituir (3.34) por sua contraparte na expressão entre colchetes de (1.24), 
tem-se:

$$
\begin{aligned}
\left\langle\boldsymbol{\varphi}^{T} \cdot \mathbb{A}_{K} \cdot \delta \mathbf{Q}\right\rangle_{s_{o}} & =\left\langle P_{1}(\boldsymbol{\varphi}), \mathcal{B}_{Q}^{\prime} \delta \mathbf{Q}\right\rangle_{s_{o}}+\left\langle\mathcal{B}^{*}(\boldsymbol{\varphi}), \mathcal{M} \delta \mathbf{Q}\right\rangle_{s_{o}} \\
& =-\left\langle\boldsymbol{\varphi}^{T} \cdot \mathbb{A}_{K} \cdot \mathcal{B}_{Q}^{\prime} \cdot \delta \mathbf{Q}\right\rangle_{s_{o}}+\left\langle\boldsymbol{\varphi}^{T} \cdot \mathcal{B}^{* T} \cdot \mathcal{M} \cdot \delta \mathbf{Q}\right\rangle_{s_{o}}
\end{aligned}
$$

Portanto,

$$
\left\langle\boldsymbol{\varphi}^{T} \cdot \mathbb{A}_{K} \cdot\left(\mathbf{I}+\mathcal{B}_{Q}^{\prime}\right) \cdot \delta \mathbf{Q}\right\rangle_{s_{o}}=\left\langle\boldsymbol{\varphi}^{T} \cdot \mathcal{B}^{* T} \cdot \mathcal{M} \cdot \delta \mathbf{Q}\right\rangle_{s_{o}}
$$

onde $\mathbf{I}$ representa a matriz identidade e a matriz $\mathcal{M}$ é dada por:

$$
\left.\mathcal{M}\right|_{s_{o}}=\sqrt{g_{11}}\left(\begin{array}{cccc}
0 & k_{x} & k_{y} & 0 \\
-u(\mathbf{u} \cdot \mathbf{n}) & k_{x} u+\mathbf{u} \cdot \mathbf{n} & k_{y} u & 0 \\
-v(\mathbf{u} \cdot \mathbf{n}) & k_{x} v & \mathbf{u} \cdot \mathbf{n}+k_{y} v & 0 \\
0 & 0 & 0 & 0
\end{array}\right)
$$

que claramente resulta em um operador que é linearmente independente de $\mathcal{B}_{Q}^{\prime}$ na eq. (3.33). Portanto, prova-se que a mesma condição de contorno adjunta nas fronteiras de saída, que mostraram-se consistentes com o fato do problema ser bem posto (HAYASHI; CEZE; VOLPE, 2013), também satisfazem os requisitos propostos por Cacuci et al. (1980).

Para finalizar a construção do problema de Riemann completo nas fronteiras de saída, três condições de contorno da eq. (3.32) devem ser combinadas com a terceira equação característca adjunta de (3.25) em um único sistema. Isso pode ser feito tanto usando as equações como são ou, alternativamente, diferenciando (3.32) com respeito ao tempo. Em princípio, a segunda opção parece permitir uma implementação mais simples e, por isso é escolhida.

Ao diferenciar a eq. (3.32) com respeito ao tempo, tem-se: $\dot{\varphi}_{\alpha}=\dot{C}_{\alpha} \varphi_{4}+C_{\alpha} \dot{\varphi}_{4}$, onde $1 \leq \alpha \leq 3$ e o ponto ( $\left(^{*}\right)$ representa $\partial() / \partial t$. Entretanto, neste contexto, os coeficientes são baseados em uma solução estacionária do escoamento, então $\dot{C}_{\alpha}=0$. Assim, o sistema completo para o problema de Riemann em fronteiras de saída pode ser escrito na forma:

$$
\left\{\begin{aligned}
\dot{\varphi}_{1}-C_{1} \dot{\varphi}_{4}= & 0 \\
\dot{\varphi}_{2}-C_{2} \dot{\varphi}_{4}= & 0 \\
\dot{\varphi}_{3}-C_{3} \dot{\varphi}_{4}= & 0 \\
\left(\sum_{\beta=1}^{3} K_{3 \beta}^{t} C_{\beta}+K_{34}^{t}\right) \dot{\varphi}_{4} & =R_{3}
\end{aligned}\right.
$$

onde as derivadas espaciais no lado direito da terceira característica na eq. (3.25) 
foram agrupadas no termo $R_{3}$. O sistema resultante é sujeito a integração numérica explicitamente.

\subsubsection{Entrada}

Assim como foi feito com as fronteiras de saída, considere a figura 3.2 e o sentido de propagação de características nas fronteiras permeáveis onde ocorre entrada de escoamento no domínio em ambos os regimes subsônico e supersônico. Novamente, isto será vital no que diz respeito às condições de contorno adjuntas.

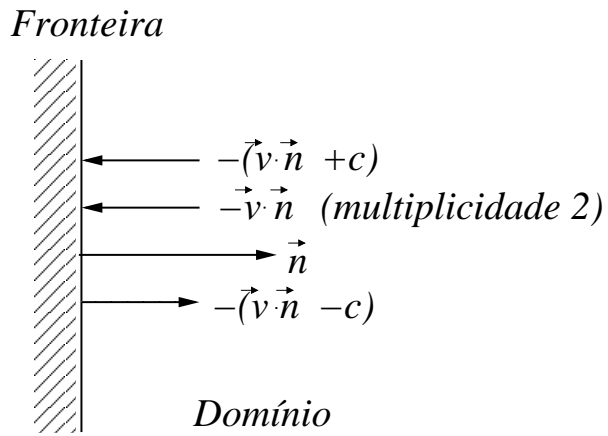

(a) Caso subsônico

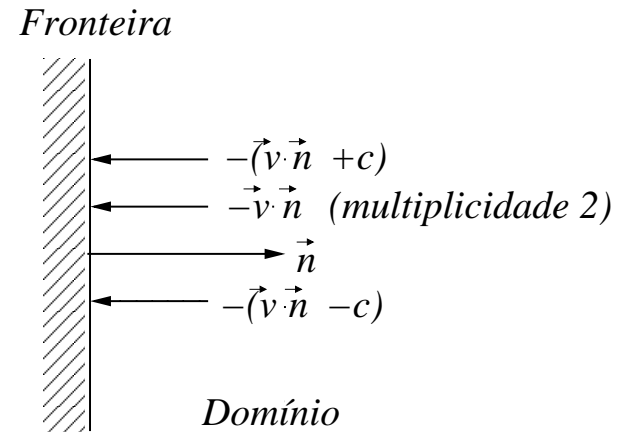

(b) Caso supersônico

Figura 3.2: Propagação de características adjuntas em fronteiras de entrada

Já que estão sujeitas a controle paramétrico, as condições de contorno de entrada são as mais complexas consideradas neste trabalho. Os dois termos responsáveis por essa fronteira na eq. (3.18) são:

$$
\begin{aligned}
\Delta_{c_{1}}+\Delta_{c_{2}} & =\left\langle\psi_{\alpha} \frac{\partial \mathcal{B}_{\alpha}}{\partial Q_{\beta}}, \delta Q_{\beta}\right\rangle_{s_{i}}+\left\langle\varphi_{\alpha} A_{\alpha \beta}^{i^{\prime}} n_{i^{\prime}}, \delta Q_{\beta}\right\rangle_{s_{i}} \\
& =\left\langle\boldsymbol{\psi} \cdot \mathcal{B}_{Q}^{\prime} \cdot \delta \mathbf{Q}\right\rangle_{s_{i}}+\left\langle\boldsymbol{\varphi}^{T} \cdot \mathbb{A}_{K} \cdot \delta \mathbf{Q}\right\rangle_{s_{i}}
\end{aligned}
$$

e eles correspondem ao produto interno $c$ na eq. (3.14). Em particular, o segundo termo $\Delta_{c_{2}}$ é um concomitante bilinear e, então, pode ser decomposto de acordo com Cacuci et al. (1980).

Em regime supersônico, as variáveis primitivas $\mathbf{V} \equiv(\rho, u, v, p)^{T}$ são todas especificadas nesse tipo de fronteira e elas fixam $\mathbf{Q}$, o que implica que $\mathcal{P} \equiv \mathbf{V}$ e $\mathcal{B}[\mathbf{Q}, \mathbf{V}]=0$. Reciprocamente, as variáveis adjuntas não são impostas em qualquer condição de contorno e $\boldsymbol{\varphi}$ vem da solução adjunta propriamente dita. Como resultado, o operador de contorno do escoamento é a matriz identidade $\mathcal{B}_{Q}^{\prime}=\mathbf{I}$ e, na falta de qualquer condição de contorno adjunta, tem-se $\mathcal{B}^{*}=0$. 
Portanto, a eq. (1.20b) toma a forma:

$$
\left(\begin{array}{llll}
1 & 0 & 0 & 0 \\
0 & 1 & 0 & 0 \\
0 & 0 & 1 & 0 \\
0 & 0 & 0 & 1
\end{array}\right)\left(\begin{array}{c}
\delta Q_{1} \\
\delta Q_{2} \\
\delta Q_{3} \\
\delta Q_{4}
\end{array}\right)=\left(\begin{array}{cccc}
1 & 0 & 0 & 0 \\
u & \rho & 0 & 0 \\
v & 0 & \rho & 0 \\
\frac{\mathbf{u} \cdot \mathbf{u}}{2} & \rho u & \rho v & \frac{1}{(\gamma-1)}
\end{array}\right)\left(\begin{array}{c}
\delta \rho \\
\delta u \\
\delta v \\
\delta p
\end{array}\right)
$$

onde a matriz quadrada do lado direito da eq. (3.40) claramente representa o operador $-\mathcal{B}_{\alpha}^{\prime} \equiv-\mathcal{B}_{V}^{\prime}$. Além disso, deve-se adicionar que, embora as variáveis $\mathbf{Q}$ e V sejam fixadas para cada solução particular do escoamento, tomadas individualmente, isso não implica que suas variações virtuais ao redor daquela solução sejam necessariamente zero. De fato, a sensibilidade do escoamento para tais variações virtuais é precisamente o principal objetivo desta investigação.

Sob tais circunstâncias, a decomposição do concomitante bilinear proposta para fronteiras de entrada supersônica se resume a:

$$
P_{1}(\boldsymbol{\varphi})=\boldsymbol{\varphi}^{T} \mathbb{A}_{K} \quad \text { e } \quad \mathcal{B}^{*}(\boldsymbol{\varphi})=0
$$

que resulta:

$$
\begin{aligned}
\left\langle\boldsymbol{\varphi}^{T} \cdot \mathbb{A}_{K} \cdot \delta \mathbf{Q}\right\rangle_{s_{i}} & =\left\langle P_{1}(\boldsymbol{\varphi}), \mathcal{B}_{Q}^{\prime} \delta \mathbf{Q}\right\rangle_{s_{i}}+\left\langle\mathcal{B}^{*}(\boldsymbol{\varphi}), \mathcal{M} \delta \mathbf{Q}\right\rangle_{s_{i}} \\
& =\left\langle P_{1}(\boldsymbol{\varphi}), \mathcal{B}_{Q}^{\prime} \delta \mathbf{Q}\right\rangle_{s_{i}} \\
& =\left\langle\boldsymbol{\varphi}^{T} \mathbb{A}_{K}, \mathbf{I} \delta \mathbf{Q}\right\rangle_{s_{i}}
\end{aligned}
$$

Uma vez que $\mathcal{B}^{*}$ é zero, o operador $\mathcal{M}$, aqui, poderia ser qualquer. Dado que é linearmente independente da matriz identidade $\mathcal{B}_{Q}^{\prime}$. Então, da eq. (1.26), pode-se resolver para $\psi$, que resulta:

$$
\boldsymbol{\psi}=-P_{1}(\boldsymbol{\varphi})=-\varphi^{T} \cdot \mathbb{A}_{K}
$$

e, da eq. (3.39), obtém-se para $\Delta_{c_{1}}+\Delta_{c_{2}}$ :

$$
\left\langle\boldsymbol{\psi} \cdot \mathcal{B}_{Q}^{\prime} \cdot \delta \mathbf{Q}\right\rangle_{s_{i}}+\left\langle\boldsymbol{\varphi}^{T} \cdot \mathbb{A}_{K} \cdot \delta \mathbf{Q}\right\rangle_{s_{i}}=-\left\langle\boldsymbol{\varphi}^{T} \cdot \mathbb{A}_{K} \cdot \mathbf{I} \cdot \delta \mathbf{Q}\right\rangle_{s_{i}}+\left\langle\boldsymbol{\varphi}^{T} \cdot \mathbb{A}_{K} \cdot \delta \mathbf{Q}\right\rangle_{s_{i}}=0
$$

Portanto, ela satisfaz os requisitos que foram propostos por Cacuci et al. (1980), a respeito da decomposição do concomitante bilinear e o cancelamento dos termos $\Delta_{c_{1}}+\Delta_{c_{2}}=0$.

Isso é absolutamente diferente das fronteiras de entrada em regime subsônico, em que apenas uma característica adjunta entra no domínio. Para uma normal apontando para dentro do domínio, ela é associada com o autovalor $-(\mathbf{u} \cdot \mathbf{n}-c)$ 
na eq. (3.21). Assim, a quarta PDE do sistema (3.25) deve ser substituída por uma condição de contorno adjunta. O escoamento, por sua vez, envolve três características que entram no domínio, desta forma requer três condições de contorno físicas.

Nas aplicações de interesse, a direção do escoamento $\theta$, juntamente com pressão de estagnação $p_{o}$ e temperatura de estagnação $T_{o}$, são prescritas. Em termos de variáveis de estado, tem-se:

$$
\left\{\begin{array}{l}
p_{o} \quad=\mathcal{B}_{1}\left(Q_{\beta}\right)=\frac{(\gamma-1)\left[2 Q_{1} Q_{4}-\left(Q_{2}^{2}+Q_{3}^{2}\right)\right]}{2 Q_{1}}\left\{1+\frac{\left(Q_{2}^{2}+Q_{3}^{2}\right)}{\gamma\left[2 Q_{1} Q_{4}-\left(Q_{2}^{2}+Q_{3}^{2}\right)\right]}\right\}^{\frac{\gamma}{(\gamma-1)}} \\
T_{o}=\mathcal{B}_{2}\left(Q_{\beta}\right)=\frac{(\gamma-1)}{2 \gamma R Q_{1}^{2}}\left[2 \gamma Q_{1} Q_{4}-(\gamma-1)\left(Q_{2}^{2}+Q_{3}^{2}\right)\right] \\
\tan (\theta)=\mathcal{B}_{3}\left(Q_{\beta}\right)=\frac{Q_{3}}{Q_{2}}
\end{array}\right.
$$

De modo a tornar a álgebra um pouco mais simples, pode-se referir a partir daqui o uso da notação $\vartheta \equiv \tan (\theta)$. Então, poderia-se escrever o sistema acima na forma da eq. (1.18b) assim:

$$
\mathcal{B}[\mathbf{Q}, \mathcal{P}]=0 \Rightarrow\left\{\begin{array}{l}
\mathcal{B}_{1}\left(Q_{\beta}\right)-p_{o}=0 \\
\mathcal{B}_{2}\left(Q_{\beta}\right)-T_{o}=0 \\
\mathcal{B}_{3}\left(Q_{\beta}\right)-\vartheta=0
\end{array}\right.
$$

onde os parâmetros de controle são precisamente formados pelo conjunto $\mathcal{P} \equiv$ $\left(p_{o}, T_{o}, \vartheta\right)^{T}$.

O teorema da função implícita (KAPLAN, 1971) pode ser utilizado par obter a forma linearizada da eq. (3.46). De modo a permanecer consistente com as derivações de Hayashi, Ceze e Volpe (2013), $\delta Q_{1}$ é tomado como o grau de liberdade livre, ou seja, a variação que é relatada para a única característica que deixa o domínio. Enquanto $\delta Q_{2}, \delta Q_{3}$ e $\delta Q_{4}$ são considerados as variações dependentes. Assim:

$$
\left\{\begin{array}{l}
\frac{\partial \mathcal{B}_{1}}{\partial Q_{2}} \delta Q_{2}+\frac{\partial \mathcal{B}_{1}}{\partial Q_{3}} \delta Q_{3}+\frac{\partial \mathcal{B}_{1}}{\partial Q_{4}} \delta Q_{4}=\delta p_{o}-\frac{\partial \mathcal{B}_{1}}{\partial Q_{1}} \delta Q_{1} \\
\frac{\partial \mathcal{B}_{2}}{\partial Q_{2}} \delta Q_{2}+\frac{\partial \mathcal{B}_{2}}{\partial Q_{3}} \delta Q_{3}+\frac{\partial \mathcal{B}_{2}}{\partial Q_{4}} \delta Q_{4}=\delta T_{o}-\frac{\partial \mathcal{B}_{2}}{\partial Q_{1}} \delta Q_{1} \\
\frac{\partial \mathcal{B}_{3}}{\partial Q_{2}} \delta Q_{2}+\frac{\partial \mathcal{B}_{3}}{\partial Q_{3}} \delta Q_{3}+\frac{\partial \mathcal{B}_{3}}{\partial Q_{4}} \delta Q_{4}=\delta \vartheta-\frac{\partial \mathcal{B}_{3}}{\partial Q_{1}} \delta Q_{1}
\end{array}\right.
$$

Sob essas condições, pode-se obter o determinante do Jacobiano principal:

$$
\Delta \equiv \frac{\partial\left(\mathcal{B}_{1}, \mathcal{B}_{2}, \mathcal{B}_{3}\right)}{\partial\left(Q_{2}, Q_{3}, Q_{4}\right)}=\left|\begin{array}{ccc}
\frac{\partial \mathcal{B}_{1}}{\partial Q_{2}} & \frac{\partial \mathcal{B}_{1}}{\partial Q_{3}} & \frac{\partial \mathcal{B}_{1}}{\partial Q_{4}} \\
\frac{\partial \mathcal{B}_{2}}{\partial Q_{2}} & \frac{\partial \mathcal{B}_{2}}{\partial Q_{3}} & \frac{\partial \mathcal{B}_{2}}{\partial Q_{4}} \\
\frac{\partial \mathcal{B}_{3}}{\partial Q_{2}} & \frac{\partial \mathcal{B}_{3}}{\partial Q_{3}} & \frac{\partial \mathcal{B}_{3}}{\partial Q_{4}}
\end{array}\right|=-\frac{(\gamma-1) M^{2} p_{o}}{\left(R \rho^{3} u^{2}\right)} \neq 0
$$


que é claramente não-nulo, logo o sistema de equações (3.47) é possível e determinado. Aplicando a regra de Cramer para resolvê-lo, tem-se:

$$
\begin{gathered}
\delta Q_{2}=\frac{1}{\Delta}\left|\begin{array}{ccc}
\delta p_{o}-\frac{\partial \mathcal{B}_{1}}{\partial Q_{1}} \delta Q_{1} & \frac{\partial \mathcal{B}_{1}}{\partial Q_{3}} & \frac{\partial \mathcal{B}_{1}}{\partial Q_{4}} \\
\delta T_{o}-\frac{\partial \mathcal{B}_{2}}{\partial Q_{1}} \delta Q_{1} & \frac{\partial \mathcal{B}_{2}}{\partial Q_{3}} & \frac{\partial \mathcal{B}_{2}}{\partial Q_{4}} \\
\delta \vartheta-\frac{\partial \mathcal{B}_{3}}{\partial Q_{1}} \delta Q_{1} & \frac{\partial \mathcal{B}_{3}}{\partial Q_{3}} & \frac{\partial \mathcal{B}_{3}}{\partial Q_{4}}
\end{array}\right| \\
\delta Q_{3}=\frac{1}{\Delta}\left|\begin{array}{ccc}
\frac{\partial \mathcal{B}_{1}}{\partial Q_{2}} & \delta p_{o}-\frac{\partial \mathcal{B}_{1}}{\partial Q_{1}} \delta Q_{1} & \frac{\partial \mathcal{B}_{1}}{\partial Q_{4}} \\
\frac{\partial \mathcal{B}_{2}}{\partial Q_{2}} & \delta T_{o}-\frac{\partial \mathcal{B}_{2}}{\partial Q_{1}} \delta Q_{1} & \frac{\partial \mathcal{B}_{2}}{\partial Q_{4}} \\
\frac{\partial \mathcal{B}_{3}}{\partial Q_{2}} & \delta \vartheta-\frac{\partial \mathcal{B}_{3}}{\partial Q_{1}} \delta Q_{1} & \frac{\partial \mathcal{B}_{3}}{\partial Q_{4}}
\end{array}\right| \\
\delta Q_{4}=\frac{1}{\Delta}\left|\begin{array}{lll}
\frac{\partial \mathcal{B}_{1}}{\partial Q_{2}} & \frac{\partial \mathcal{B}_{1}}{\partial Q_{3}} & \delta p_{o}-\frac{\partial \mathcal{B}_{1}}{\partial Q_{1}} \delta Q_{1} \\
\frac{\partial \mathcal{B}_{2}}{\partial Q_{2}} & \frac{\partial \mathcal{B}_{2}}{\partial Q_{3}} & \delta T_{o}-\frac{\partial \mathcal{B}_{2}}{\partial Q_{1}} \delta Q_{1} \\
\frac{\partial \mathcal{B}_{3}}{\partial Q_{2}} & \frac{\partial \mathcal{B}_{3}}{\partial Q_{3}} & \delta \vartheta-\frac{\partial \mathcal{B}_{3}}{\partial Q_{1}} \delta Q_{1}
\end{array}\right|
\end{gathered}
$$

Ou ainda:

$$
\begin{aligned}
& \delta Q_{2}=\frac{1}{\Delta}\left|\begin{array}{ccc}
1 & \frac{\partial \mathcal{B}_{1}}{\partial Q_{3}} & \frac{\partial \mathcal{B}_{1}}{\partial Q_{4}} \\
0 & \frac{\partial \mathcal{B}_{2}}{\partial Q_{3}} & \frac{\partial \mathcal{B}_{2}}{\partial Q_{4}} \\
0 & \frac{\partial \mathcal{B}_{3}}{\partial Q_{3}} & \frac{\partial \mathcal{B}_{3}}{\partial Q_{4}}
\end{array}\right| \delta p_{o}+\frac{1}{\Delta}\left|\begin{array}{ccc}
0 & \frac{\partial \mathcal{B}_{1}}{\partial Q_{3}} & \frac{\partial \mathcal{B}_{1}}{\partial Q_{4}} \\
1 & \frac{\partial \mathcal{B}_{2}}{\partial Q_{3}} & \frac{\partial \mathcal{B}_{2}}{\partial Q_{4}} \\
0 & \frac{\partial \mathcal{B}_{3}}{\partial Q_{3}} & \frac{\partial \mathcal{B}_{3}}{\partial Q_{4}}
\end{array}\right| \delta T_{o}+ \\
& +\frac{1}{\Delta}\left|\begin{array}{ccc}
0 & \frac{\partial \mathcal{B}_{1}}{\partial Q_{3}} & \frac{\partial \mathcal{B}_{1}}{\partial Q_{4}} \\
0 & \frac{\partial \mathcal{B}_{2}}{\partial Q_{3}} & \frac{\partial \mathcal{B}_{2}}{\partial Q_{4}} \\
1 & \frac{\partial \mathcal{B}_{3}}{\partial Q_{3}} & \frac{\partial \mathcal{B}_{3}}{\partial Q_{4}}
\end{array}\right| \delta \vartheta-\frac{1}{\Delta}\left|\begin{array}{ccc}
\frac{\partial \mathcal{B}_{1}}{\partial Q_{1}} & \frac{\partial \mathcal{B}_{1}}{\partial Q_{3}} & \frac{\partial \mathcal{B}_{1}}{\partial Q_{4}} \\
\frac{\partial \mathcal{B}_{2}}{\partial Q_{1}} & \frac{\partial \mathcal{B}_{2}}{\partial Q_{3}} & \frac{\partial \mathcal{B}_{2}}{\partial Q_{4}} \\
\frac{\partial \mathcal{B}_{3}}{\partial Q_{1}} & \frac{\partial \mathcal{B}_{3}}{\partial Q_{3}} & \frac{\partial \mathcal{B}_{3}}{\partial Q_{4}}
\end{array}\right| \delta Q_{1} \\
& \delta Q_{3}=\frac{1}{\Delta}\left|\begin{array}{ccc}
\frac{\partial \mathcal{B}_{1}}{\partial Q_{2}} & 1 & \frac{\partial \mathcal{B}_{1}}{\partial Q_{4}} \\
\frac{\partial \mathcal{B}_{2}}{\partial Q_{2}} & 0 & \frac{\partial \mathcal{B}_{2}}{\partial Q_{4}} \\
\frac{\partial \mathcal{B}_{3}}{\partial Q_{2}} & 0 & \frac{\partial \mathcal{B}_{3}}{\partial Q_{4}}
\end{array}\right| \delta p_{o}+\frac{1}{\Delta}\left|\begin{array}{ccc}
\frac{\partial \mathcal{B}_{1}}{\partial Q_{2}} & 0 & \frac{\partial \mathcal{B}_{1}}{\partial Q_{4}} \\
\frac{\partial \mathcal{B}_{2}}{\partial Q_{2}} & 1 & \frac{\partial \mathcal{B}_{2}}{\partial Q_{4}} \\
\frac{\partial \mathcal{B}_{3}}{\partial Q_{2}} & 0 & \frac{\partial \mathcal{B}_{3}}{\partial Q_{4}}
\end{array}\right| \delta T_{o}+ \\
& +\frac{1}{\Delta}\left|\begin{array}{ccc}
\frac{\partial \mathcal{B}_{1}}{\partial Q_{2}} & 0 & \frac{\partial \mathcal{B}_{1}}{\partial Q_{4}} \\
\frac{\partial \mathcal{B}_{2}}{\partial Q_{2}} & 0 & \frac{\partial \mathcal{B}_{2}}{\partial Q_{4}} \\
\frac{\partial \mathcal{B}_{3}}{\partial Q_{2}} & 1 & \frac{\partial \mathcal{B}_{3}}{\partial Q_{4}}
\end{array}\right| \delta \vartheta-\frac{1}{\Delta}\left|\begin{array}{ccc}
\frac{\partial \mathcal{B}_{1}}{\partial Q_{2}} & \frac{\partial \mathcal{B}_{1}}{\partial Q_{1}} & \frac{\partial \mathcal{B}_{1}}{\partial Q_{4}} \\
\frac{\partial \mathcal{B}_{2}}{\partial Q_{2}} & \frac{\partial \mathcal{B}_{2}}{\partial Q_{1}} & \frac{\partial \mathcal{B}_{2}}{\partial Q_{4}} \\
\frac{\partial \mathcal{B}_{3}}{\partial Q_{2}} & \frac{\partial \mathcal{B}_{3}}{\partial Q_{1}} & \frac{\partial \mathcal{B}_{3}}{\partial Q_{4}}
\end{array}\right| \delta Q_{1} \\
& \delta Q_{4}=\frac{1}{\Delta}\left|\begin{array}{ccc}
\frac{\partial \mathcal{B}_{1}}{\partial Q_{2}} & \frac{\partial \mathcal{B}_{1}}{\partial Q_{3}} & 1 \\
\frac{\partial \mathcal{B}_{2}}{\partial Q_{2}} & \frac{\partial \mathcal{B}_{2}}{\partial Q_{3}} & 0 \\
\frac{\partial \mathcal{B}_{3}}{\partial Q_{2}} & \frac{\partial \mathcal{B}_{3}}{\partial Q_{3}} & 0
\end{array}\right| \delta p_{o}+\frac{1}{\Delta}\left|\begin{array}{ccc}
\frac{\partial \mathcal{B}_{1}}{\partial Q_{2}} & \frac{\partial \mathcal{B}_{1}}{\partial Q_{3}} & 0 \\
\frac{\partial \mathcal{B}_{2}}{\partial Q_{2}} & \frac{\partial \mathcal{B}_{2}}{\partial Q_{3}} & 1 \\
\frac{\partial \mathcal{B}_{3}}{\partial Q_{2}} & \frac{\partial \mathcal{B}_{3}}{\partial Q_{3}} & 0
\end{array}\right| \delta T_{o}+ \\
& +\frac{1}{\Delta}\left|\begin{array}{ccc}
\frac{\partial \mathcal{B}_{1}}{\partial Q_{2}} & \frac{\partial \mathcal{B}_{1}}{\partial Q_{3}} & 0 \\
\frac{\partial \mathcal{B}_{2}}{\partial Q_{2}} & \frac{\partial \mathcal{B}_{2}}{\partial Q_{3}} & 0 \\
\frac{\partial \mathcal{B}_{3}}{\partial Q_{2}} & \frac{\partial \mathcal{B}_{3}}{\partial Q_{3}} & 1
\end{array}\right| \delta \vartheta-\frac{1}{\Delta}\left|\begin{array}{ccc}
\frac{\partial \mathcal{B}_{1}}{\partial Q_{2}} & \frac{\partial \mathcal{B}_{1}}{\partial Q_{3}} & \frac{\partial \mathcal{B}_{1}}{\partial Q_{1}} \\
\frac{\partial \mathcal{B}_{2}}{\partial Q_{2}} & \frac{\partial \mathcal{B}_{2}}{\partial Q_{3}} & \frac{\partial \mathcal{B}_{2}}{\partial Q_{1}} \\
\frac{\partial \mathcal{B}_{3}}{\partial Q_{2}} & \frac{\partial \mathcal{B}_{3}}{\partial Q_{3}} & \frac{\partial \mathcal{B}_{3}}{\partial Q_{1}}
\end{array}\right| \delta Q_{1}
\end{aligned}
$$


Alternativamente, as diferenciais totais para as variações dependentes são computadas na forma:

$$
\begin{aligned}
& \delta Q_{2}=\frac{1}{\Delta}\left[\frac{\partial\left(\mathcal{B}_{1}, \mathcal{B}_{2}, \mathcal{B}_{3}\right)}{\partial\left(p_{o}, Q_{3}, Q_{4}\right)} \delta p_{o}+\frac{\partial\left(\mathcal{B}_{1}, \mathcal{B}_{2}, \mathcal{B}_{3}\right)}{\partial\left(T_{o}, Q_{3}, Q_{4}\right)} \delta T_{o}+\frac{\partial\left(\mathcal{B}_{1}, \mathcal{B}_{2}, \mathcal{B}_{3}\right)}{\partial\left(\vartheta, Q_{3}, Q_{4}\right)} \delta \vartheta-\frac{\partial\left(\mathcal{B}_{1}, \mathcal{B}_{2}, \mathcal{B}_{3}\right)}{\partial\left(Q_{1}, Q_{3}, Q_{4}\right)} \delta Q_{1}\right] \\
& \delta Q_{3}=\frac{1}{\Delta}\left[\frac{\partial\left(\mathcal{B}_{1}, \mathcal{B}_{2}, \mathcal{B}_{3}\right)}{\partial\left(Q_{2}, p_{o}, Q_{4}\right)} \delta p_{o}+\frac{\partial\left(\mathcal{B}_{1}, \mathcal{B}_{2}, \mathcal{B}_{3}\right)}{\partial\left(Q_{2}, T_{o}, Q_{4}\right)} \delta T_{o}+\frac{\partial\left(\mathcal{B}_{1}, \mathcal{B}_{2}, \mathcal{B}_{3}\right)}{\partial\left(Q_{2}, \vartheta, Q_{4}\right)} \delta \vartheta-\frac{\partial\left(\mathcal{B}_{1}, \mathcal{B}_{2}, \mathcal{B}_{3}\right)}{\partial\left(Q_{2}, Q_{1}, Q_{4}\right)} \delta Q_{1}\right] \\
& \delta Q_{4}=\frac{1}{\Delta}\left[\frac{\partial\left(\mathcal{B}_{1}, \mathcal{B}_{2}, \mathcal{B}_{3}\right)}{\partial\left(Q_{2}, Q_{3}, p_{o}\right)} \delta p_{o}+\frac{\partial\left(\mathcal{B}_{1}, \mathcal{B}_{2}, \mathcal{B}_{3}\right)}{\partial\left(Q_{2}, Q_{3}, T_{o}\right)} \delta T_{o}+\frac{\partial\left(\mathcal{B}_{1}, \mathcal{B}_{2}, \mathcal{B}_{3}\right)}{\partial\left(Q_{2}, Q_{3}, \vartheta\right)} \delta \vartheta-\frac{\partial\left(\mathcal{B}_{1}, \mathcal{B}_{2}, \mathcal{B}_{3}\right)}{\partial\left(Q_{2}, Q_{3}, Q_{1}\right)} \delta Q_{1}\right]
\end{aligned}
$$

Os resultados são então rearranjados como na eq. $(1.20 \mathrm{~b}), \mathcal{B}_{Q}^{\prime} \cdot \delta \mathbf{Q}=-\mathcal{B}_{\mathcal{P}}^{\prime}$. $\delta \mathcal{P}:$

$$
\left(\begin{array}{cccc}
0 & 0 & 0 & 0 \\
\frac{\partial Q_{2}}{\partial Q_{1}} & -1 & 0 & 0 \\
\frac{\partial Q_{3}}{\partial Q_{1}} & 0 & -1 & 0 \\
\frac{\partial Q_{4}}{\partial Q_{1}} & 0 & 0 & -1
\end{array}\right)\left(\begin{array}{c}
\delta Q_{1} \\
\delta Q_{2} \\
\delta Q_{3} \\
\delta Q_{4}
\end{array}\right)=-\left(\begin{array}{ccc}
0 & 0 & 0 \\
\frac{\partial Q_{2}}{\partial p_{o}} & \frac{\partial Q_{2}}{\partial T_{o}} & \frac{\partial Q_{2}}{\partial \vartheta} \\
\frac{\partial Q_{3}}{\partial p_{o}} & \frac{\partial Q_{3}}{\partial T_{o}} & \frac{\partial Q_{3}}{\partial \vartheta} \\
\frac{\partial Q_{4}}{\partial p_{o}} & \frac{\partial Q_{4}}{\partial T_{o}} & \frac{\partial Q_{4}}{\partial \vartheta}
\end{array}\right)\left(\begin{array}{c}
\delta p_{o} \\
\delta T_{o} \\
\delta \vartheta
\end{array}\right)
$$

onde as derivadas parciais vem do determinante Jacobiano correspondente nos diferenciais totais, eq. (3.55), i.e.:

$$
\begin{aligned}
& \frac{\partial Q_{2}}{\partial Q_{1}}=-\frac{\frac{\partial\left(\mathcal{B}_{1}, \mathcal{B}_{2}, \mathcal{B}_{3}\right)}{\partial\left(Q_{1}, Q_{3}, Q_{4}\right)}}{\frac{\partial\left(\mathcal{B}_{1}, \mathcal{B}_{2}, \mathcal{B}_{3}\right)}{\partial\left(Q_{2}, Q_{3}, Q_{4}\right)}} ; \frac{\partial Q_{3}}{\partial Q_{1}}=-\frac{\frac{\partial\left(\mathcal{B}_{1}, \mathcal{B}_{2}, \mathcal{B}_{3}\right)}{\partial\left(Q_{2}, Q_{1}, Q_{4}\right)}}{\frac{\partial\left(\mathcal{B}_{1}, \mathcal{B}_{2}, \mathcal{B}_{3}\right)}{\partial\left(Q_{2}, Q_{3}, Q_{4}\right)}} ; \frac{\partial Q_{4}}{\partial Q_{1}}=-\frac{\frac{\partial\left(\mathcal{B}_{1}, \mathcal{B}_{2}, \mathcal{B}_{3}\right)}{\partial\left(Q_{2}, Q_{3}, Q_{1}\right)}}{\frac{\partial\left(\mathcal{B}_{1}, \mathcal{B}_{2}, \mathcal{B}_{3}\right)}{\partial\left(Q_{2}, Q_{3}, Q_{4}\right)}} \\
& \frac{\partial Q_{2}}{\partial p_{o}}=\frac{\frac{\partial\left(\mathcal{B}_{1}, \mathcal{B}_{2}, \mathcal{B}_{3}\right)}{\partial\left(p_{o}, Q_{3}, Q_{4}\right)}}{\frac{\partial\left(\mathcal{B}_{1}, \mathcal{B}_{2}, \mathcal{B}_{3}\right)}{\partial\left(Q_{2}, Q_{3}, Q_{4}\right)}} ; \frac{\partial Q_{3}}{\partial p_{o}}=\frac{\frac{\partial\left(\mathcal{B}_{1}, \mathcal{B}_{2}, \mathcal{B}_{3}\right)}{\partial\left(Q_{2}, p_{o}, Q_{4}\right)}}{\frac{\partial\left(\mathcal{B}_{1}, \mathcal{B}_{2}, \mathcal{B}_{3}\right)}{\partial\left(Q_{2}, Q_{3}, Q_{4}\right)}} ; \frac{\partial Q_{4}}{\partial p_{o}}=\frac{\frac{\partial\left(\mathcal{B}_{1}, \mathcal{B}_{2}, \mathcal{B}_{3}\right)}{\partial\left(Q_{2}, Q_{3}, p_{o}\right)}}{\frac{\partial\left(\mathcal{B}_{1}, \mathcal{B}_{2}, \mathcal{B}_{3}\right)}{\partial\left(Q_{2}, Q_{3}, Q_{4}\right)}} \\
& \frac{\partial Q_{2}}{\partial T_{o}}=\frac{\frac{\partial\left(\mathcal{B}_{1}, \mathcal{B}_{2}, \mathcal{B}_{3}\right)}{\partial\left(T_{o}, Q_{3}, Q_{4}\right)}}{\frac{\partial\left(\mathcal{B}_{1}, \mathcal{B}_{2}, \mathcal{B}_{3}\right)}{\partial\left(Q_{2}, Q_{3}, Q_{4}\right)}} ; \frac{\partial Q_{3}}{\partial T_{o}}=\frac{\frac{\partial\left(\mathcal{B}_{1}, \mathcal{B}_{2}, \mathcal{B}_{3}\right)}{\partial\left(Q_{2}, T_{o}, Q_{4}\right)}}{\frac{\partial\left(\mathcal{B}_{1}, \mathcal{B}_{2}, \mathcal{B}_{3}\right)}{\partial\left(Q_{2}, Q_{3}, Q_{4}\right)}} ; \frac{\partial Q_{4}}{\partial T_{o}}=\frac{\frac{\partial\left(\mathcal{B}_{1}, \mathcal{B}_{2}, \mathcal{B}_{3}\right)}{\partial\left(Q_{2}, Q_{3}, T_{o}\right)}}{\frac{\partial\left(\mathcal{B}_{1}, \mathcal{B}_{2}, \mathcal{B}_{3}\right)}{\partial\left(Q_{2}, Q_{3}, Q_{4}\right)}} \\
& \frac{\partial Q_{2}}{\partial \vartheta}=\frac{\frac{\partial\left(\mathcal{B}_{1}, \mathcal{B}_{2}, \mathcal{B}_{3}\right)}{\partial\left(\theta, Q_{3}, Q_{4}\right)}}{\frac{\partial\left(\mathcal{B}_{1}, \mathcal{B}_{2}, \mathcal{B}_{3}\right)}{\partial\left(Q_{2}, Q_{3}, Q_{4}\right)}} ; \frac{\partial Q_{3}}{\partial \vartheta}=\frac{\frac{\partial\left(\mathcal{B}_{1}, \mathcal{B}_{2}, \mathcal{B}_{3}\right)}{\partial\left(Q_{2}, \theta, Q_{4}\right)}}{\frac{\partial\left(\mathcal{B}_{1}, \mathcal{B}_{2}, \mathcal{B}_{3}\right)}{\partial\left(Q_{2}, Q_{3}, Q_{4}\right)}} ; \frac{\partial Q_{4}}{\partial \vartheta}=\frac{\frac{\partial\left(\mathcal{B}_{1}, \mathcal{B}_{2}, \mathcal{B}_{3}\right)}{\partial\left(Q_{2}, Q_{3}, \theta\right)}}{\frac{\partial\left(\mathcal{B}_{1}, \mathcal{B}_{2}, \mathcal{B}_{3}\right)}{\partial\left(Q_{2}, Q_{3}, Q_{4}\right)}}
\end{aligned}
$$

Os operadores acima são dados explicitamente por:

$$
\begin{gathered}
\mathcal{B}_{Q}^{\prime}=\left(\begin{array}{cccc}
0 & 0 & 0 & 0 \\
u-\frac{\gamma p u}{\rho\left(u^{2}+v^{2}\right)} & -1 & 0 & 0 \\
v-\frac{\gamma p v}{\rho\left(u^{2}+v^{2}\right)} & 0 & -1 & 0 \\
\frac{-\rho u^{2}+\gamma\left(-2(-2+\gamma) p+\rho u^{2}\right)+(-1+\gamma) \rho v^{2}}{2(-1+\gamma) \rho} & 0 & 0 & -1
\end{array}\right) \\
\mathcal{B}_{\mathcal{P}}^{\prime}=\left(\begin{array}{ccc}
0 & 0 & 0 \\
\frac{\rho u}{M^{2} P_{o}} & \frac{u}{2 T_{o}}\left(\rho-\frac{2 \rho}{M^{2}}\right) & -\frac{\rho^{2} u^{2} v}{\gamma M^{2} p} \\
\frac{\rho v}{M^{2} P_{o}} & \frac{v}{2 T_{o}}\left(\rho-\frac{2 \rho}{M^{2}}\right) & \frac{\rho^{2} u^{3}}{\gamma M^{2} p} \\
\frac{(\gamma-1) p}{P_{o}} & \frac{\gamma p}{T}\left[\frac{1}{(\gamma-1)}-\frac{T}{T_{o}}\right] & 0
\end{array}\right)
\end{gathered}
$$


onde a primeira linha de ambos os operadores significa simplesmente que $\delta Q_{1}$ é livre, de uma forma que essa variação representa os efeitos de variações arbitrárias do domínio do escoamento na fronteira.

Agora, o ponto mais importante desta pesquisa está no fato de que o operador $\mathcal{B}_{Q}^{\prime}$ acima é exatamente equivalente a eq. (43) de Hayashi, Ceze e Volpe (2013), que é reproduzida abaixo para efeito de comparação:

$$
\left\{\begin{array}{l}
\delta Q_{2}=\frac{u}{2}\left[2-\gamma+\gamma^{2}-\frac{2 e(\gamma-1) \gamma}{\left(u^{2}+v^{2}\right)}\right] \delta Q_{1} \\
\delta Q_{3}=\frac{v}{2}\left[2-\gamma+\gamma^{2}-\frac{2 e(\gamma-1) \gamma}{\left(u^{2}+v^{2}\right)}\right] \delta Q_{1} \\
\delta Q_{4}=\frac{1}{2}\left[-2 e(\gamma-2) \gamma+(\gamma-1)^{2}\left(u^{2}+v^{2}\right)\right] \delta Q_{1}
\end{array}\right.
$$

Com pequeno esforço algébrico, percebe-se que a única diferença entre elas é o fato de que os coeficientes de $\delta Q_{1}$ e seus correspondentes elementos em $\mathcal{B}_{Q}^{\prime}$ estão escritos em termos da pressão estática $p$ na eq. (3.58), enquanto na eq. (3.60) são dados em termos da energia $e$.

O fato de que ambas as formas (3.58) e (3.60) são inteiramente equivalentes tem uma consequencia fundamental: ele implica que as mesmas condições de contorno implementadas naquele trabalho, podem ser utilizadas aqui na forma de um operador $\mathcal{B}^{*}(\boldsymbol{\varphi})=0$. A forma original dessa condição dada pelas eqs. (45) e (46) daquele trabalho (HAYASHI; CEZE; VOLPE, 2013) é dada por:

$$
C_{1} \varphi_{1}+C_{2} \varphi_{2}+C_{3} \varphi_{3}+C_{4} \varphi_{4}=0
$$

onde os coeficientes são:

$$
\left\{\begin{array}{l}
C_{1}=\left[2-\gamma+\gamma^{2}-\frac{2 \gamma e(\gamma-1)}{u^{2}+v^{2}}\right] \frac{\left(k_{x} u+k_{y} v\right)}{2} \\
C_{2}=\frac{\left(2-\gamma+\gamma^{2}\right) k_{x} u^{2}+2\left(1-\gamma+\gamma^{2}\right) k_{y} u v}{2}-\frac{(\gamma-1) \gamma k_{x} v^{2}}{2}-\frac{2 \gamma e(\gamma-1)\left[2 k_{y} u v+k_{x}\left(u^{2}-v^{2}\right)\right]}{2\left(u^{2}+v^{2}\right)} \\
C_{3}=\frac{-\gamma(\gamma-1) k_{y} u^{4}+2 k_{y} u^{2} v^{2}}{2\left(u^{2}+v^{2}\right)}+\frac{2\left(1-\gamma+\gamma^{2}\right) k_{x}\left(u^{3} v+u v^{3}\right)+\left(2-\gamma+\gamma^{2}\right) k_{y} v^{4}+2 \gamma e(\gamma-1)\left[k_{y}\left(u^{2}-v^{2}\right)-2 k_{x} u v\right]}{2\left(u^{2}+v^{2}\right)} \\
C_{4}=-\frac{\left(k_{x} u+k_{y} v\right)\left[(\gamma-1)\left(u^{2}+v^{2}\right)-2 \gamma e\right]\left[\left(2-\gamma+\gamma^{2}\right)\left(u^{2}+v^{2}\right)-2 \gamma e(\gamma-1)\right]}{4\left(u^{2}+v^{2}\right)}
\end{array}\right.
$$

A forma da eq. (3.61) em termos do operador $\mathcal{B}^{*}$ é bem simples e é dada por:

$$
\left.\mathcal{B}^{*}\right|_{s_{i}}=\left(\begin{array}{cccc}
1 & -\frac{C_{2}}{C_{1}} & -\frac{C_{3}}{C_{1}} & -\frac{C_{4}}{C_{1}} \\
0 & 0 & 0 & 0 \\
0 & 0 & 0 & 0 \\
0 & 0 & 0 & 0
\end{array}\right)
$$


que é consistente com o fato de que existe apenas uma característica adjunta entrando no domínio em fronteiras de entrada subsônica. As últimas três linhas, na realidade, representam as características que apontam para fora do domínio.

Então, ao aplicar à condição de entrada a mesma decomposição apresentada na eq. (3.34),

$$
\left\{\begin{array}{l}
P_{1}(\boldsymbol{\varphi})=-\boldsymbol{\varphi}^{T} \mathbb{A}_{K} \\
\mathcal{B}^{*}(\boldsymbol{\varphi})=\boldsymbol{\varphi}^{T} \mathcal{B}^{* T}
\end{array}\right.
$$

A álgebra resultante é inteiramente análoga aquela da eq. (3.36), como esperado. A única diferença é que os produtos internos agora se referem à condição de entrada $\left(s_{i}\right)$, ao invés daquela de saída $\left(s_{o}\right)$. Além dessa mudança, o operador $\mathcal{M}$ é então obtido:

$\left.\mathcal{M}\right|_{s_{i}}=\sqrt{g_{11}}\left(\begin{array}{cccc}M_{11} & 0 & 0 & 0 \\ 0 & 0 & 0 & 0 \\ 0 & 0 & 0 & 0 \\ 0 & 0 & 0 & 0\end{array}\right) ; M_{11}=\left(\gamma^{2}-\gamma+2\right)\left(k_{x}+k_{y}\right)-\frac{e_{t}(\gamma-1) \gamma\left(k_{x} u+k_{y} v\right)}{\left(u^{2}+v^{2}\right)}$

que também é linearmente independente do $\mathcal{B}_{Q}^{\prime}$ correspondente, como pode ser claramente visto ao comparar o resultado acima com a eq. (3.58). A partir da primeira equação em (3.64) e da (1.26), obtém-se $\psi$ :

$$
\boldsymbol{\psi}=-P_{1}(\boldsymbol{\varphi})=+\boldsymbol{\varphi}^{T} \cdot \mathbb{A}_{K}
$$

Finalmente, levando em conta o fato de que $\mathcal{B}^{*}(\varphi)=\varphi^{T} \mathcal{B}^{* T}=0$, e substituindo as eqs. (3.64) e (3.66) para os termos correspondentes na eq. (3.39), obtém-se para $\Delta_{c_{1}}+\Delta_{c_{2}}$ :

$$
\begin{aligned}
\left\langle\boldsymbol{\psi} \cdot \mathcal{B}_{Q}^{\prime} \cdot \delta \mathbf{Q}\right\rangle_{s_{i}}+\left\langle\boldsymbol{\varphi}^{T} \cdot \mathbb{A}_{K} \cdot \delta \mathbf{Q}\right\rangle_{s_{i}} & =\left\langle\boldsymbol{\psi} \cdot \mathcal{B}_{Q}^{\prime} \cdot \delta \mathbf{Q}\right\rangle_{s_{i}}+\left\langle P_{1}(\boldsymbol{\varphi}), \mathcal{B}_{Q}^{\prime} \cdot \delta \mathbf{Q}\right\rangle_{s_{i}} \\
& =\left\langle\boldsymbol{\varphi}^{T} \cdot \mathbb{A}_{K} \cdot \mathcal{B}_{Q}^{\prime} \cdot \delta \mathbf{Q}\right\rangle_{s_{i}}-\left\langle\boldsymbol{\varphi}^{T} \cdot \mathbb{A}_{K} \cdot \mathcal{B}_{Q}^{\prime} \cdot \delta \mathbf{Q}\right\rangle_{s_{i}} \\
& =0
\end{aligned}
$$

que resulta identicamente nula, como certamente deveria.

Acima de tudo, o que esses resultados mostram, olhando o tratamento das fronteiras de farfield no problema de contorno de Hayashi, Ceze e Volpe (2013), é que, não apenas ele é totalmente consistente com os requisitos de Cacuci et al. (1980), como também permite estender o escopo das derivadas de sensibilidade. Isso parece indicar que o trabalho de Hayashi, Ceze e Volpe (2013) gira ao redor de casos mais simples, onde ambas as eqs. (3.40) e (3.56) tem $\delta \mathcal{P}=0$ na seção 
de entrada.

O problema de Riemann completo é obtido ao unir as as três primeiras PDE's características adjuntas da eq. (3.25) com a única condição de contorno adjunta das eqs. (3.61) e (3.62).

Ao diferenciar (3.61) com respeito ao tempo, tem-se: $\dot{C}_{\alpha} \varphi_{\alpha}+C_{\alpha} \dot{\varphi}_{\alpha}=0$, onde $1 \leq \alpha \leq 4$. Novamente os coeficientes da eq. (3.62) são baseados na solução de estado estacionário do escoamento, então $\dot{C}_{\alpha}=0$. Então, ao incluir a equação resultante no sistema de PDE's características a que saem do domínio, obtém-se o sistema completo de equações:

$$
\left\{\begin{array}{l}
K_{11}^{t} \frac{\partial \varphi_{1}}{\partial t}+K_{12}^{t} \frac{\partial \varphi_{2}}{\partial t}+K_{13}^{t} \frac{\partial \varphi_{3}}{\partial t}+K_{14}^{t} \frac{\partial \varphi_{4}}{\partial t}=R_{1} \\
K_{21}^{t} \frac{\partial \varphi_{1}}{\partial t}+K_{22}^{t} \frac{\partial \varphi_{2}}{\partial t}+K_{23}^{t} \frac{\partial \varphi_{3}}{\partial t}+K_{24}^{t} \frac{\partial \varphi_{4}}{\partial t}=R_{2} \\
K_{31}^{t} \frac{\partial \varphi_{1}}{\partial t}+K_{32}^{t} \frac{\partial \varphi_{2}}{\partial t}+K_{33}^{t} \frac{\partial \varphi_{3}}{\partial t}+K_{34}^{t} \frac{\partial \varphi_{4}}{\partial t}=R_{3} \\
C_{1} \frac{\partial \varphi_{1}}{\partial t}+C_{2} \frac{\partial \varphi_{2}}{\partial t}+C_{3} \frac{\partial \varphi_{3}}{\partial t}+C_{4} \frac{\partial \varphi_{4}}{\partial t}=0
\end{array}\right.
$$

onde as derivadas espaciais do lado direito da eq. (3.25) tiveram seus termos coletados em $R_{\alpha}$. O sistema resultante é sujeito então a integração numérica com marcha explícita no tempo.

A tabela 3.2 apresenta um resumo das condições de contorno adjuntas nas fronteiras de entrada que correspondem a cada regime de escoamento.

Tabela 3.2: Resumo das condições de contorno adjuntas de entrada

\begin{tabular}{ccc}
\hline \hline Regime & Variação $\delta \mathbf{Q}$ & Condição adjunta $\varphi$ \\
\hline subsônico & $\delta Q_{2}\left(\delta Q_{1}\right), \delta Q_{3}\left(\delta Q_{1}\right), \delta Q_{4}\left(\delta Q_{1}\right)$ & $\begin{array}{c}\varphi_{1}\left(\varphi_{2}, \varphi_{3}, \varphi_{4}\right) \\
\text { eq. }(3.60)\end{array}$ \\
\hline supersônico & $\delta Q_{\alpha}=0$ & $\varphi_{\alpha}$ livres \\
\hline \hline
\end{tabular}

\subsection{Gradiente Adjunto}

A forma original do gradiente de sensibilidades vem da eq. (3.14), e corresponde aos termos $e, g, h$ e $i$, que são reproduzidos abaixo:

$$
\begin{aligned}
\langle\mathbf{a}, \delta \boldsymbol{\alpha}\rangle & =-\left\langle\varphi_{\alpha}, \delta\left(J \beta_{i^{\prime}}^{2}\right) f_{\alpha}^{i^{\prime}} n_{2}\right\rangle_{b_{\infty}}+\left\langle\delta\left(J \beta_{i^{\prime}}^{k}\right), \frac{f_{\alpha}^{i^{\prime}}}{J} \frac{\partial\left(J \varphi_{\alpha}\right)}{\partial \xi^{k}}\right\rangle+ \\
& -\left\langle\mathcal{F}, \delta\left|\frac{\mathrm{d} S^{\prime}}{\mathrm{d} S}\right|\right\rangle_{b_{w}}-\left\langle p,\left[\varphi_{\left(i^{\prime}+1\right)} \delta\left(J \beta_{i^{\prime}}^{2}\right) n_{2}\right]\right\rangle_{b_{w}}-\left\langle\boldsymbol{\psi}, \mathcal{B}_{\mathcal{P}}^{\prime} \delta \mathcal{P}\right\rangle_{s_{i}}(3 .
\end{aligned}
$$


Entre eles, os quatro primeiros termos do lado direito da equação representam variações geométricas e podem ser simplificados ainda mais, como mostra Jameson e Kim (2003b) no apêndice . O último termo da equação acima é o único que, na realidade trata de sensibilidades nas seções de entrada. Na ausência de variações de geometria, ele se torna o único termo no gradiente,

$$
\langle\mathbf{a}, \delta \mathcal{P}\rangle=-\left\langle\boldsymbol{\psi}, \mathcal{B}_{\mathcal{P}}^{\prime} \delta \mathcal{P}\right\rangle_{s_{i}}
$$

Para escoamentos supersônicos, o operador $\mathcal{B}_{\mathcal{P}}^{\prime}$ vem da eq. (3.40), enquanto $\boldsymbol{\psi}$ é dado pela eq. (3.43). Assim, obtém-se:

$$
\left\{\begin{array}{l}
\frac{\partial I}{\partial \rho}=\int_{s_{i}}\left(-U_{n} \varphi_{1}-u U_{n} \varphi_{2}-v U_{n} \varphi_{3}-\frac{1}{2} c^{2} U_{n} M^{2} \varphi_{4}\right) \mathrm{d} S \\
\frac{\partial I}{\partial u}=\int_{s_{i}}\left[-k_{x} \rho \varphi_{1}-\rho\left(k_{x} u+U_{n}\right) \varphi_{2}-\rho v k_{x} \varphi_{3}+\left(-k_{x} \sigma-\rho u U_{n}\right) \varphi_{4}\right] \mathrm{d} S \\
\frac{\partial I}{\partial v}=\int_{s_{i}}\left[-k_{y} \rho \varphi_{1}-\rho u k_{y} \varphi_{2}-\rho\left(k_{y} v+U_{n}\right) \varphi_{3}+\left(-k_{y} \sigma-\rho v U_{n}\right) \varphi_{4}\right] \mathrm{d} S \\
\frac{\partial I}{\partial p}=\int_{s_{i}}\left(-k_{x} \varphi_{2}-k_{y} \varphi_{3}-\frac{\gamma U_{n}}{\gamma-1} \varphi_{4}\right) \mathrm{d} S
\end{array}\right.
$$

onde $\sigma \equiv \frac{c^{2}\left[2+(\gamma-1) M^{2}\right] \rho}{2(\gamma-1)}$ foi definida de modo a apresentar uma forma mais "limpa" das equações e $U_{n}=k_{x} u+k_{y} v$, corresponde a velocidade do fluido normal a fronteira.

Similarmente, no caso de entrada subsônica, $\mathcal{B}_{\mathcal{P}}^{\prime}$ é dado pela eq. (3.59), enquanto $\boldsymbol{\psi}$ vem da eq. (3.66). Desta forma:

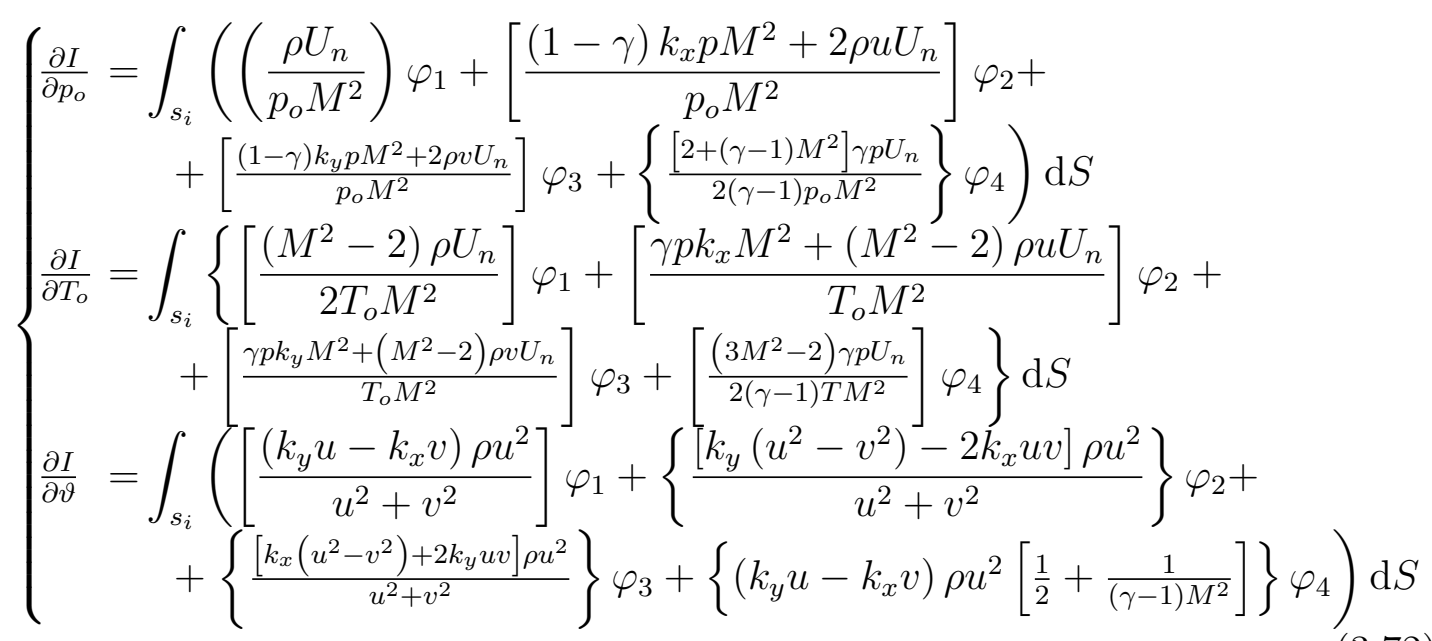

A respeito das equações dadas acima, vale acrescentar que nenhuma das expressões depende explicitamente da medida de mérito, ou seja, na realidade, toda influência do funcional aparece apenas por meio da solução adjunta. 


\section{RESULTADOS}

Este capítulo apresenta os resultados e discussões das simulações numéricas realizadas com o objetivo de validar o código computacional implementado que resolve as equações adjuntas, bem como as expressões para o cálculo de sensibilidades não-geométricas desenvolvidas no capítulo 3. Os testes são realizados nos regimes de escoamento subsônico, supersônico e transônico em 4 geometrias: um bocal divergente, um perfil diamante, um perfil NACA 0012 e um perfil RAE 2822 .

\subsection{Método de Validação da Solução Adjunta}

A ausência de significado físico das variáveis adjuntas dificulta a validação de um código computacional que resolva estas equações visualizando apenas o seu campo de solução. Isso ocorre porque não há como determiná-las empirica e/ou analiticamente em qualquer situação. Uma das formas comumente utilizadas para contornar esse problema é comparar diretamente os gradientes determinados a partir da solução adjunta com resultados computados por outro método numérico. Apesar de custoso, o método de diferenças finitas, brevemente citado na seção 1.4.2 e também referenciado na literatura como método de força bruta, é adequado para essa finalidade devido a simplicidade de sua implementação.

Formalmente, derivadas de uma função $f(x)$ em um ponto $x_{o}$ podem ser estimadas por diferenças finitas com segunda ordem de precisão ${ }^{1}$ ao considerar as seguintes expansões em série de Taylor em torno de $x_{o}$ :

$$
\begin{aligned}
& f\left(x_{o}+\Delta x\right)=f\left(x_{o}\right)+\Delta x \frac{\mathrm{d} f\left(x_{o}\right)}{\mathrm{d} x}+\frac{(\Delta x)^{2}}{2 !} \frac{\mathrm{d}^{2} f\left(x_{o}\right)}{\mathrm{d} x^{2}}+\cdots \\
& f\left(x_{o}-\Delta x\right)=f\left(x_{o}\right)-\Delta x \frac{\mathrm{d} f\left(x_{o}\right)}{\mathrm{d} x}+\frac{(\Delta x)^{2}}{2 !} \frac{\mathrm{d}^{2} f\left(x_{o}\right)}{\mathrm{d} x^{2}}+\cdots
\end{aligned}
$$

Subtraindo a eq. (4.2) de (4.1) e rearranjando os termos de maneira conve-

\footnotetext{
${ }^{1}$ Matematicamente, um método numérico é dito de $k$-ésima ordem se o erro da solução $e$ for proporcional à dimensão da malha $h$ elevado a $k$-ésima potência, isto é, $e \propto h^{k}$.
} 
niente, obtém-se a seguinte expressão para a sensibilidade de $f$ com relação à $x$ em $x=x_{o}$ :

$$
\left.\frac{\mathrm{d} f(x)}{\mathrm{d} x}\right|_{x_{o}}=\frac{f\left(x_{o}+\Delta x\right)-f\left(x_{o}-\Delta x\right)}{2 \Delta x}+\mathcal{O}\left(\Delta x^{2}\right)
$$

Assim, a avaliação da sensibilidade de uma determinada medida de mérito, I, com relação a cada parâmetro necessita de duas simulações convergidas do escoamento, uma para avaliar $I\left(x_{o}+\Delta x\right)$ e outra para $I\left(x_{o}-\Delta x\right)$.

Como já comentado, o regime de velocidade de escoamentos compressíveis modelados pelas equações de Euler define o número de propriedades que podem ser impostas como condições de contorno no domínio computacional. Em escoamentos bidimensionais, em particular, três propriedades são impostas nas fronteiras de entrada quando o escoamento é subsônico nesta região, enquanto quatro propriedades são impostas no caso supersônico. Assim, a escolha dos parâmetros de controle deste trabalho naturalmente recai sobre essas propriedades fixadas no problema físico. Portanto, para a avaliação dos gradientes $\partial I / \partial \rho, \partial I / \partial u, \partial I / \partial v$ e $\partial I / \partial p$ com a ordem de precisão desejada no caso supersônico, são necessárias oito simulações do escoamento. Enquanto isso, o caso subsônico requer seis soluções convergidas para a obtenção de $\partial I / \partial p_{o}, \partial I / \partial T_{o}$ e $\partial I / \partial \vartheta$. Novamente, vale destacar que em ambos os regimes de escoamento descritos, a mesma informação pode ser obtida apenas com uma solução física e uma adjunta.

\subsection{Medidas de Mérito Adotadas}

As simulações apresentadas neste capítulo comparam gradientes de três medidas de mérito distintas. Todas elas são integrais de superfície nas paredes sólidas do domínio computacional como mostra a eq. (4.4). Nos casos de escoamento externo elas correspondem às forças de sustentação $(L)$, arrasto de onda ${ }^{2}(D)$ e momento de arfagem em torno do bordo de ataque $(M)$.

$$
\begin{aligned}
L & =\oint_{b_{w}}\left(-p n_{y} \cos \theta+p n_{x} \sin \theta\right) \mathrm{d} S \\
D & =\oint_{b_{w}}\left(-p n_{x} \cos \theta-p n_{y} \sin \theta\right) \mathrm{d} S \\
M & =\oint_{b_{w}}\left[-p n_{x}\left(y-y_{r e f}\right) \mathrm{d}-p n_{y}\left(x-x_{r e f}\right)\right] \mathrm{d} S
\end{aligned}
$$

\footnotetext{
${ }^{2}$ Vale lembrar que o modelo adotado para representar as equações que governam o escoamento no âmbito deste trabalho é invíscido e, portanto, só é possível computar arrasto de onda nas soluções do problema primal.
} 
onde $\vec{n}=n_{x} \hat{\imath}+n_{y} \hat{\jmath}$ é o versor normal à parede apontando para dentro do domínio, o ponto $\left(x_{r e f}, y_{r e f}\right)$ corresponde às coordenadas de referência para o cálculo do momento e $\theta$ corresponde ao ângulo de incidência do escoamento não perturbado nas fronteiras de entrada.

As seções subsequentes retratam testes de validação do método proposto para calcular sensibilidades não-geométricas. A seção 4.3 apresenta os casos de escoamento interno em um bocal divergente. Nos testes com tal geometria, apenas sensibilidades com relação à medida de mérito dada pela eq. (4.4a) são computadas. Já nos casos de escoamento externo descritos na seção 4.4, são avaliados os gradientes das forças de sustentação e arrasto de onda quando o regime de escoamento é supersônico e da força de sustentação e momento de arfagem nos testes em regime de escoamento subsônico/transônico. Para tanto, utiliza-se de códigos computacionais que resolvem os sistemas de PDE's de ambos os problemas primal e dual por meio do método de volumes finitos com discretização das equações do movimento em um esquema centrado nas células (cell centered) com dissipação artificial. A solução de estado estacionário é obtida por meio de marcha no tempo da solução transiente via Runge-Kutta de 5 passos com $2^{\text {a }}$ ordem de precisão (JAMESON; SCHMIDT; TURKEL, 1981), em malhas não-estruturadas formadas por elementos triangulares (JAMESON; SRIRAM; MARTINELLI, 2003), com condições de contorno baseadas em equações diferenciais características e relações de compatibilidade (HAYASHI; CEZE; VOLPE, 2013). A forma adimensional das propriedades do escoamento são obtidas com base em um estado de referência (HAYASHI, 2009), que é o mesmo para todos os testes realizados neste capítulo. Os valores de referência são: massa específica, $\rho_{r e f}=1.486 \mathrm{~kg} / \mathrm{m}^{3}$; velocidade, $v_{r e f}=320.44 \mathrm{~m} / \mathrm{s}$; e temperatura, $T_{r e f}=357.77 \mathrm{~K}$. Além disso, a razão de calores específicos $\gamma=1.4$ e constante do gás $R=287 \mathrm{~J} / \mathrm{kg} \cdot \mathrm{K}$ também são comuns a todas as simulações aqui presentes.

\subsection{Escoamento Interno}

Como primeiro teste de validação, optou-se por estudar o escoamento em um bocal divergente. A escolha por essa geometria se deu devido a possibilidade de analisar escoamentos em qualquer regime, subsônico ou supersônico sem a presença de ondas de choque. A parede é descrita pela parábola $y=0.3 x^{2}+0.3$, com $x$ variando de 0 a 1 . A linha de simetria, por sua vez é dada por $y=0$. 


\subsubsection{Caso Supersônico}

As simulações nesse regime do escoamento partiram de condições de entrada e saída de escoamento idênticas, que foram definidas com pressão estática $p=108.9877 \mathrm{kPa}$, temperatura estática $T=255.5 \mathrm{~K}$ e número de Mach variando de 1.5 até 2.5. A figura 4.1 apresenta a malha do bocal divergente com 9002 elementos triangulares que foi utilizada nas simulações dos problemas físicos e adjunto, juntamente com contornos de número de Mach no caso em que $M=2$ na seção de entrada.

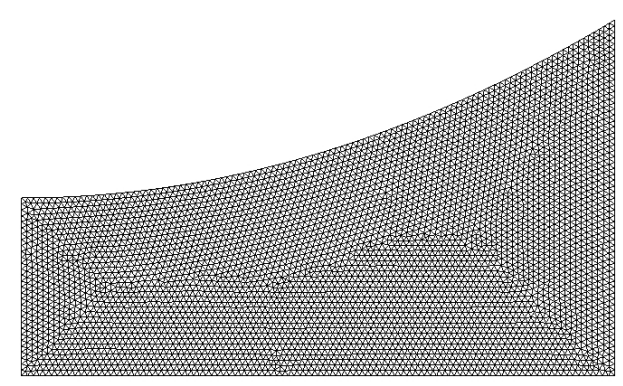

(a) Malha

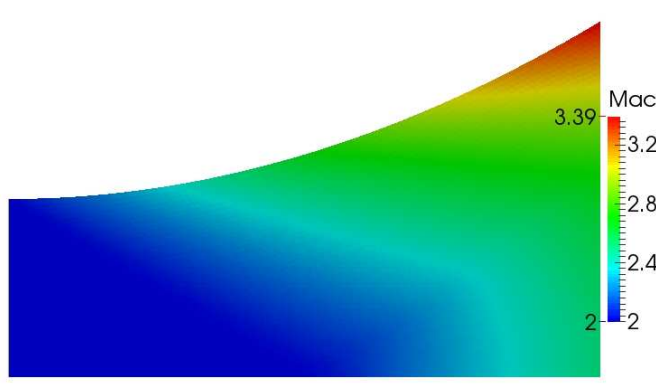

(b) Contornos de número de Mach

Figura 4.1: Escoamento supersônico interno em bocal divergente com número de Mach, $M=2$, na seção de entrada.

A título de ilustração, a solução adjunta referente ao mesmo escoamento é exibida, em suas quatro variáveis, na fig. 4.2. Ela é, particularmente, interessante para ratificar o argumento relatado no início deste capítulo, de que, na ausência de solução analítica, é difícil validar o código que resolve o problema dual apenas com base no seu campo de solução $\varphi_{\alpha}$. Para entender isso, basta observar que, diferentemente de pressão ou velocidade, os multiplicadores de Lagrange são entidades matemáticas abstratas e que não há meios de medi-los diretamente na natureza.

Os gradientes adjuntos da medida de mérito dada pela eq. (4.4a) com relação às variáveis primitivas são finalmente computados e, para fins de validação, comparados com os determinados por diferenças finitas na fig. 4.3. Nota-se que em todos eles há uma excelente concordância entre os resultados de ambos os métodos. As diferenças são apresentadas na fig. 4.4 e permanecem sempre abaixo de $4.6 \times 10^{-4}$ para todos os componentes do gradiente.

Aqui vale lembrar que a solução adjunta tem aproximadamente o mesmo custo computacional das equações que governam o escoamento. Assim, o tempo total gasto para que se possa computar o gradiente utilizando o método adjunto foi 


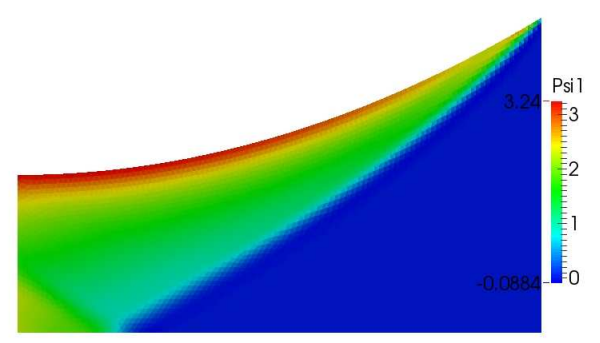

(a) $\varphi_{1}$

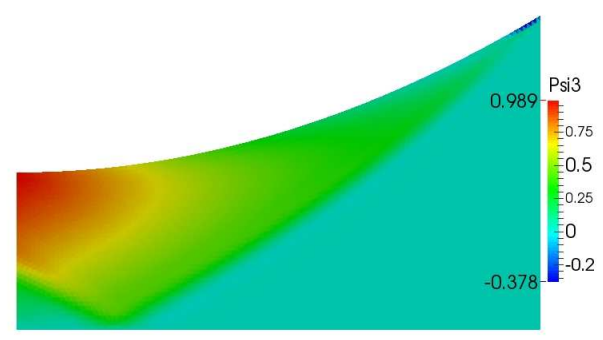

(c) $\varphi_{3}$

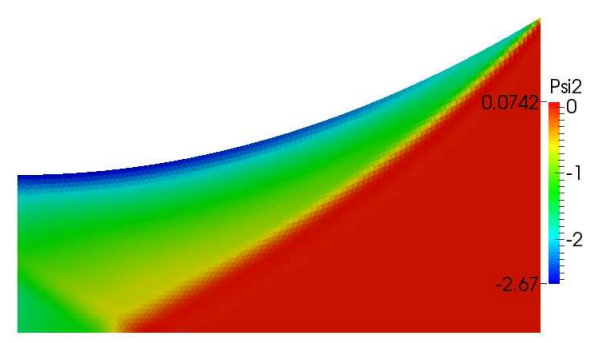

(b) $\varphi_{2}$

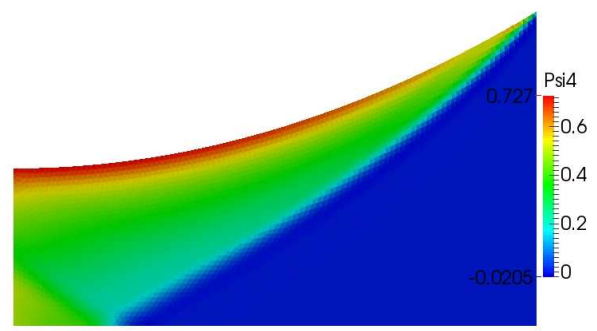

(d) $\varphi_{4}$

Figura 4.2: Solução adjunta em bocal divergente supersônico com medida de mérito $I=L$ e número de Mach, $M=2$, na seção de entrada.

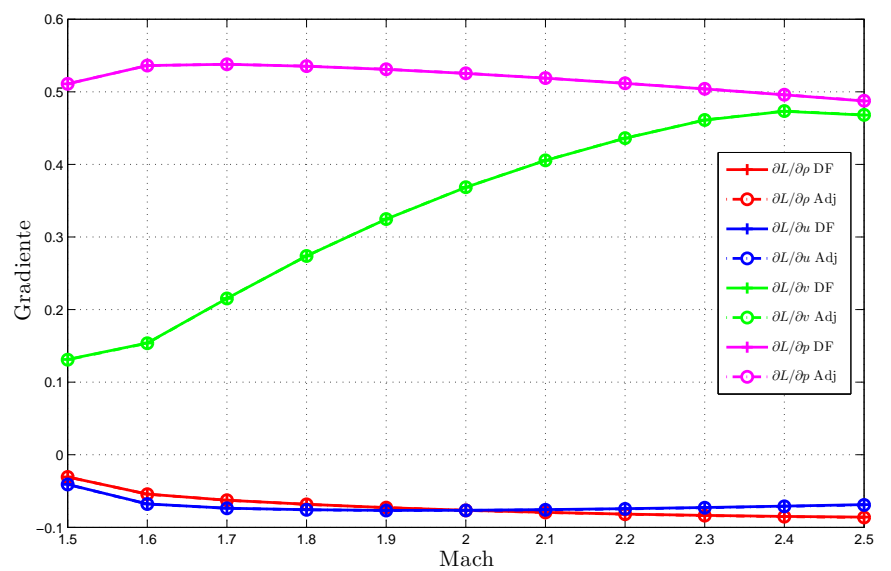

Figura 4.3: Sensibilidade em bocal supersônico. Linhas sólidas com marcador ' + ', diferenças finitas; traço-ponto com marcador 'o', adjunto. Vermelho, $\partial L / \partial \rho$; azul, $\partial L / \partial u$; verde, $\partial L / \partial v$; magenta, $\partial L / \partial p$.

de aproximadamente duas simulações, enquanto através do método de diferenças finitas com segunda ordem de precisão, foram necessárias oito simulações para conseguir a mesma informação. Isso destaca a importância do método para estimar gradientes de sensibilidades já que o seu custo é praticamente indpendente do número de parâmetros de controle. Nesse sentido, a figura 4.5 compara os históricos de convergência da soluções de ambos os problemas no caso em que $M=2$ na seção de entrada. É fácil perceber que ambos os problemas convergem 


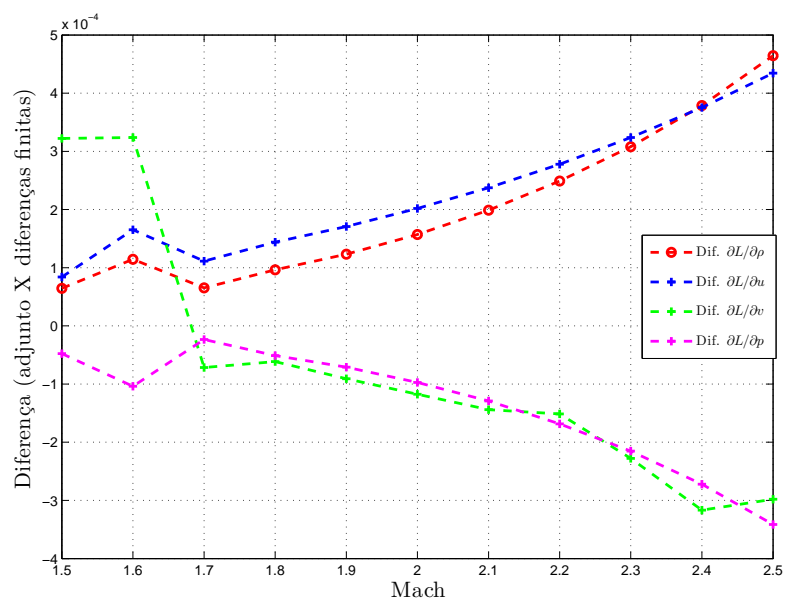

Figura 4.4: Diferenças entre os gradientes de sensibilidade em bocal supersônico obtidos pelo método adjunto e diferenças finitas. Vermelho, $\partial L / \partial \rho$; azul, $\partial L / \partial u$; verde, $\partial L / \partial v$; magenta, $\partial L / \partial p$.

seguindo o mesmo padrão.
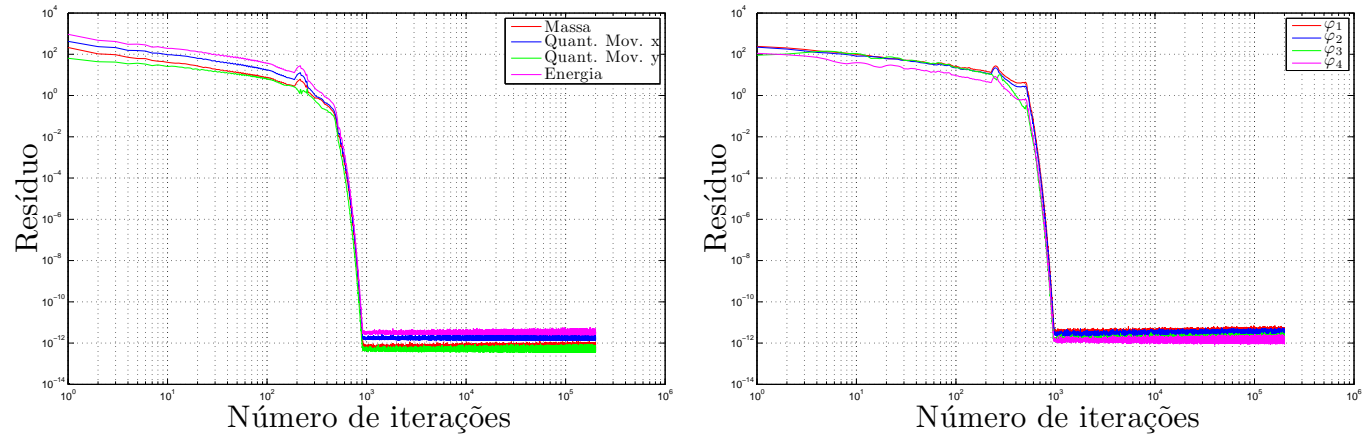

(a) Problema físico. Vermelho, massa; azul, (b) Problema adjunto. Vermelho, $\varphi_{1}$; azul, quant. mov. x; verde, quant. mov. y; ma- $\varphi_{2}$; verde, $\varphi_{3}$; magenta, $\varphi_{4}$. genta, energia.

Figura 4.5: Comparação dos históricos de convergência dos problemas físico e adjunto em bocal divergente sob regime de escoamento supersônico $(M=2)$.

\subsubsection{Caso Subsônico}

Antes de definir as condições iniciais aplicadas nas simulações no regime de escoamento subsônico, vale salientar que o sistema de equações governantes se torna mal condicionado à medida em que se aproxima do limite incompressível (algo em torno de $M=0.3$ ). Isto ocorre porque a equação de conservação da energia passa a ser redundante quando os efeitos de compressibilidade desaparecem. Dessa forma, em todos os resultados apresentados nesta tese, as soluções iniciais do problema físico tem número de Mach $M \geq 0.5$. Observe, no entanto, que entre as propriedades escolhidas como condições de contorno do problema 
físico nas seções de entrada, não está incluso o número de Mach neste trabalho. Isso quer dizer que este é um grau de liberdade livre nessa conjuntura. Logo, mesmo que a solução inicial tenha, por exemplo, $M=0.5$ nesta fronteira, não implica que a solução estacionária o terá.

A tabela 4.1 apresenta, justamente, as condições de contorno físicas impostas nas fronteiras de entrada e saída. As propriedades já estão com seus valores adimensionalizados pelo estado de referência já citado.

Tabela 4.1: Condições de contorno impostas em bocal divergente subsônico (propriedades adimensionais)

\begin{tabular}{|c|c|c|c|c|}
\hline \multicolumn{4}{|c|}{ Entrada } & Saída \\
\hline Caso & $p_{o}$ & $T_{o}$ & $\theta$ & $p$ \\
\hline 1 & 0.847295 & 0.75 & $0^{\circ}$ & 0.818591 \\
\hline 2 & 0.911074 & 0.765714 & $0^{\circ}$ & 0.871403 \\
\hline 3 & 0.990787 & 0.784286 & $0^{\circ}$ & 0.939453 \\
\hline 4 & 1.088814 & 0.805714 & $0^{\circ}$ & 1.025695 \\
\hline 5 & 1.208074 & 0.83 & $0^{\circ}$ & 1.133591 \\
\hline
\end{tabular}

A comparação entre gradientes para os casos listados na tabela 4.1 são mostrados na fig. 4.6. Os casos 1 a 5 correspondem, respectivamente, aos seguintes valores de Mach na seção de entrada da solução convergida: 0.47, 0.55, 0.64, 0.715 e 0.75. Estes equivalem a abscissa da figura 4.6, que compara as sensibilidades obtidas por diferenças finitas e pelo método adjunto.

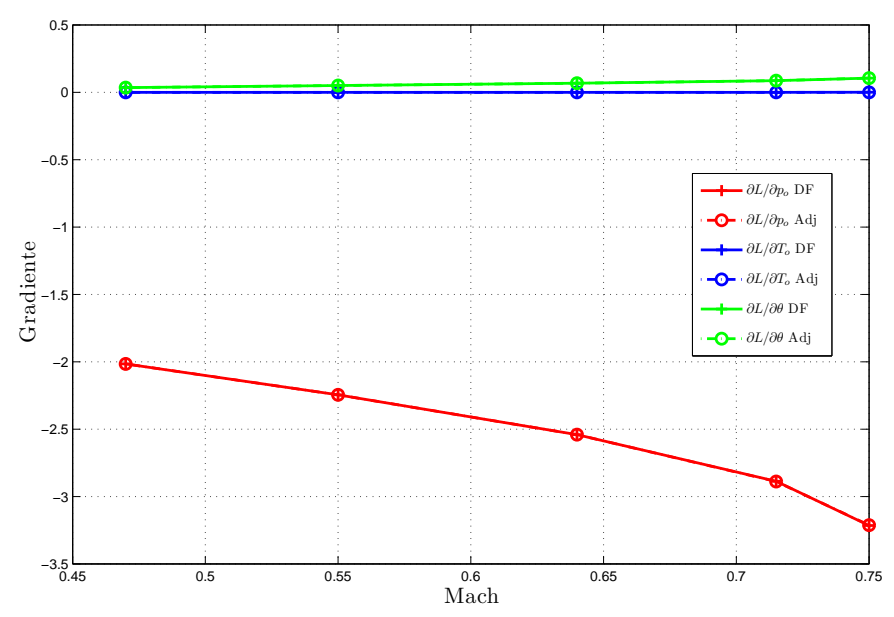

Figura 4.6: Sensibilidade em bocal subsônico. Linhas sólidas com marcador '+', diferenças finitas; traço-ponto com marcador 'o', adjunto. Vermelho, $\partial L / \partial p_{o}$; azul, $\partial L / \partial T_{o}$; verde, $\partial L / \partial \theta$.

É importante ressaltar que a geometria escolhida é conveniente também para 
o caso subsônico, pois o escoamento se mantém sempre no mesmo regime, i.e., não existem quaisquer regiões do domínio onde o escoamento ultrapassa Mach 1. As diferenças entre os gradientes se mantém todas menores do que $3.0 \times 10^{-3} \mathrm{em}$ todos os seus componentes, como indica a fig. 4.7 .

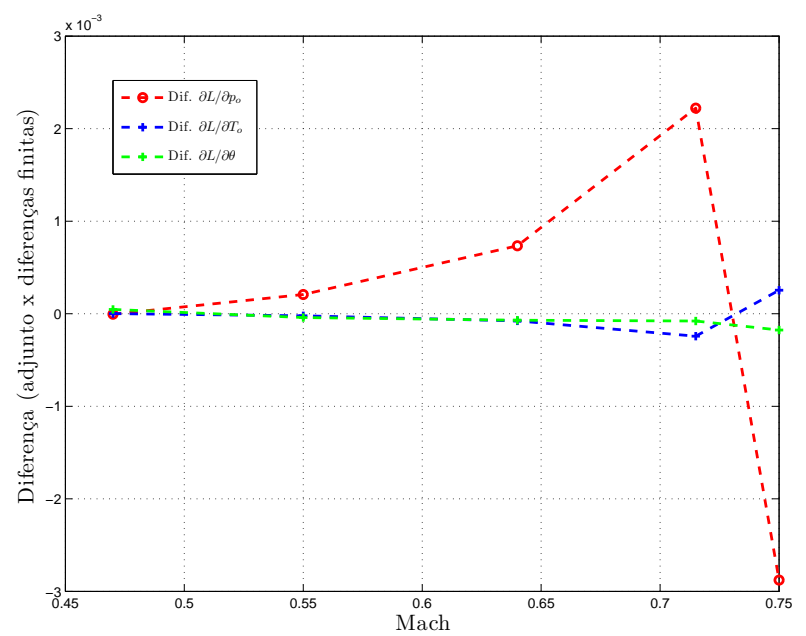

Figura 4.7: Diferenças entre as sensibilidade em bocal subsônico obtidos pelo método adjunto e diferenças finitas. Vermelho, $\partial L / \partial p_{o}$; azul, $\partial L / \partial T_{o}$; verde, $\partial L / \partial \theta$.

Os históricos de convergência das soluções física e adjunta do caso 4 da tabela 4.1 são mostrados na figura 4.8. Note que, a velocidade de com que a solução converge neste caso é bem menor do que no caso supersônico apresentado na figura 4.5 mesmo com ambas serem simuladas na mesma malha computacional.
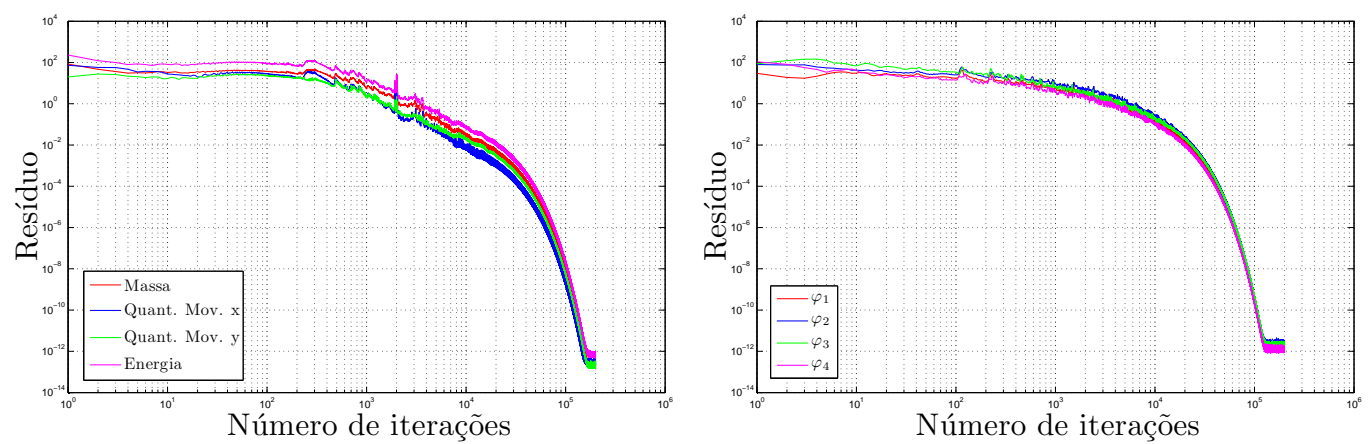

(a) Problema físico. Vermelho, massa; azul, (b) Problema adjunto. Vermelho, $\varphi_{1}$; azul, quant. mov. $\mathrm{x}$; verde, quant. mov. $\mathrm{y} ;$ ma- $\varphi_{2}$; verde, $\varphi_{3}$; magenta, $\varphi_{4}$. genta, energia.

Figura 4.8: Comparação dos históricos de convergência dos problemas físico e adjunto em bocal divergente em regime de escoamento subsônico (caso 4). 


\subsection{Escoamento Externo}

Tipicamente, escoamentos em torno de superfícies aerodinâmicas em escoamentos externos são simuladas em malhas "O" ou "C", como as apresentadas na fig. 4.9. Aqui, no entanto, há um problema em utilizar esses tipos de malha, particularmente, em escoamentos no regime supersônico. Note, que nessas malhas, ocorreriam regiões em que a velocidade normal à esse tipo de fronteira permeável seriam menores do que a velocidade do som. Nesse caso, não seria possível, por exemplo, computar as variações por diferenças finitas, já que a física presente seria diferente ao longo dessa fronteira, ou seja, haveriam regiões em que três propriedades seriam fixadas, enquanto em outras teriam quatro.

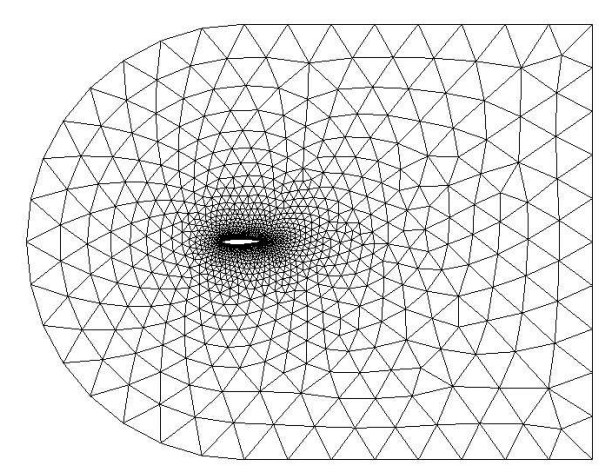

(a) Malha "C"

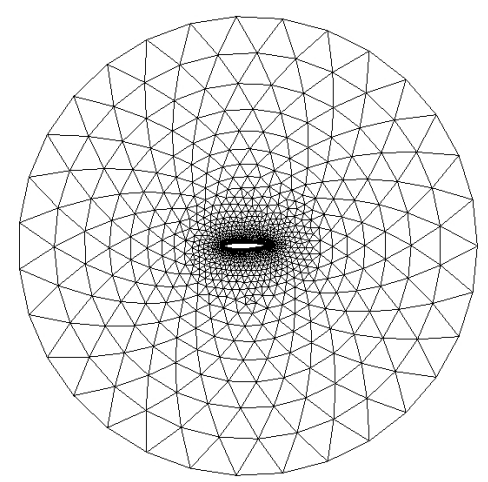

(b) Malha "O"

Figura 4.9: Malhas tipicamente utilizadas para simular escoamentos externos em torno de aerofólios.

Por esses motivos, optou-se por um tipo de malha com formato quadrado e condições periódicas nas fronteiras superior e inferior, como indica a fig 4.10. Nas malhas utilizadas em todos os casos de escoamento externo, o lado do quadrado foi definido como sendo 100 vezes a corda dos aerofólios, assim sendo as fronteiras esquerda e direita foram implementadas com condições de contorno de farfield.

\subsubsection{Perfil Diamante}

As simulações em escoamentos internos da seção 4.3 se mostraram suficientes para provar o conceito apresentado neste trabalho em escoamentos compressíveis nos regimes subsônico e supersônico sem a presença de ondas de choque. Seguindo uma sequencia crescente de complexidade dos problmas analisados, é natural investigar casos em que ocorram choques. Como uma primeira geometria para estudar o comportamento da solução adjunta nessas condições, optou-se por um perfil diamante com vértices nos pontos coordenados $(0.0 ; 0.0),(0.5 ; 0.05)$, 


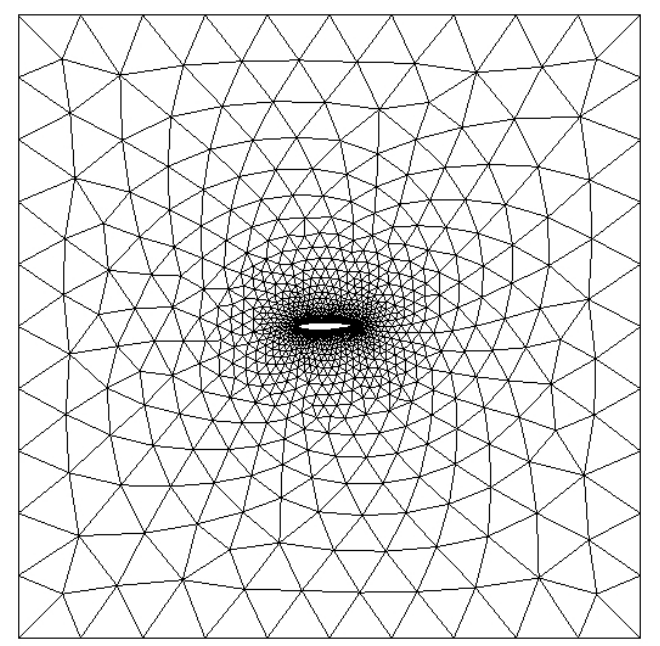

Figura 4.10: Malha com condições de contorno periódicas nas fronteiras superior e inferior.

$(0.5 ;-0.05)$ e $(1.0 ; 0.0)$. A figura 4.11 mostra a malha em que foram processados os resultados nessa geometria, juntamente com contornos de número de Mach, no caso em que se tem número de Mach de escoamento livre, $M_{\infty}=1.8$, e ângulo de ataque de $2^{\circ}$.

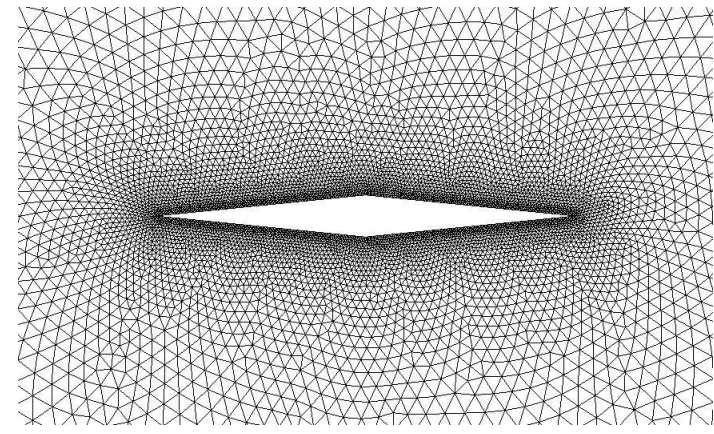

(a) Malha

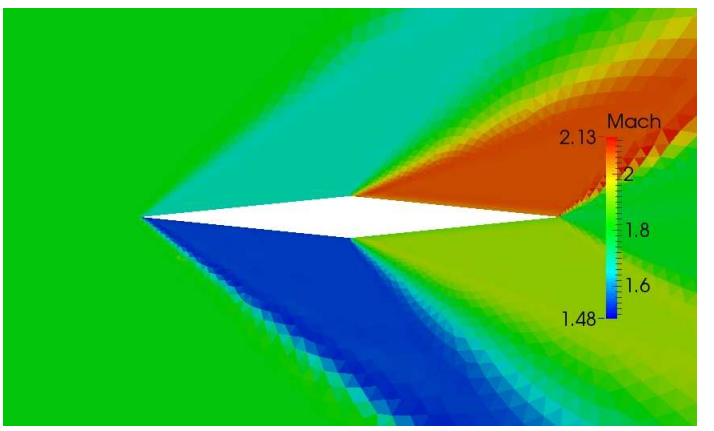

(b) Contorno de Mach

Figura 4.11: Escoamento supersônico externo em perfil diamante com número de Mach de escoamento livre, $M_{\infty}=1.8$, e ângulo de ataque, $\theta=2^{\circ}$.

Todos os casos de validação desta seção foram simulados com a malha da figura 4.11(a), formada por 19926 elementos, sendo 400 deles definindo a geometria do perfil. A figura 4.11(b) ainda revela uma característica desejada para iniciar as investigações em escoamentos onde aparecem ondas de choque: a garantia de que não haverá choque destacado. Ao observar a figura C.1 no apêndice C, é possível encontrar o ângulo de deflexão máximo para cada condição de escoamento aqui simulada. Veja que para $1.5 \leq M \leq 2.5$, tem-se $12^{\circ} \leq \theta_{\max } \leq 30^{\circ}$. Logo, com ângulo de deflexão da geometria escolhida (aproximadamente $5.7^{\circ}$ ) é sempre menor do que $\theta_{\max }$ e, portanto, todas as ondas de choque que surgirem nesses 
escoamentos estarão coladas à ponta do perfil, como explica Anderson (2011).

A figura 4.12, por sua vez, apresenta a solução adjunta do escoamento mostrado na figura 4.11 quando a medida de mérito adotada é a força de sustentação $(L)$. É interessante notar a presença de um "choque adjunto" com inclinação contrária se comparada com o choque físico. Esse fato surge da complementaridade entre os problemas primal e dual amplamente discutido por Hayashi (2009) e que está diretamente relacionado com a direção de propagação de suas características, em sentidos opostos.

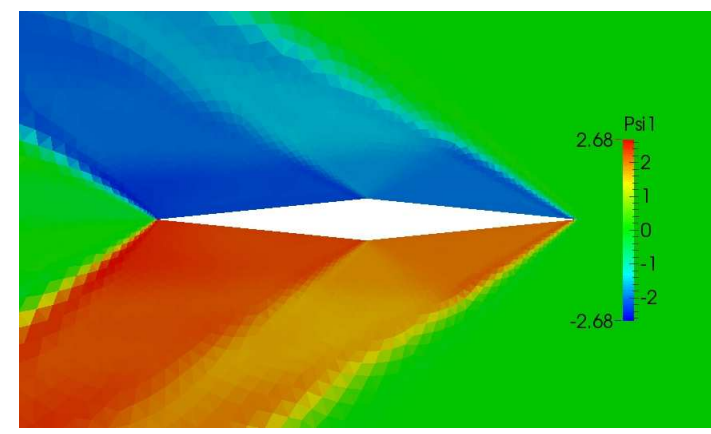

(a) $\varphi_{1}$

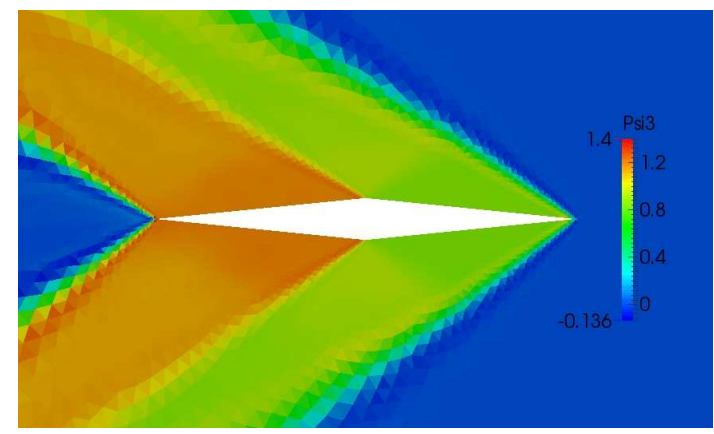

(c) $\varphi_{3}$

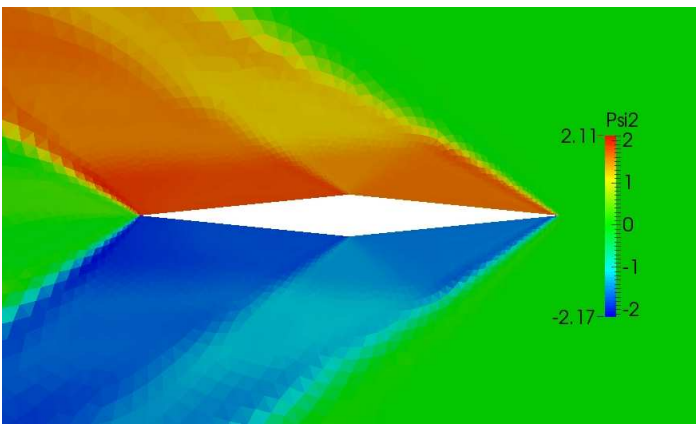

(b) $\varphi_{2}$

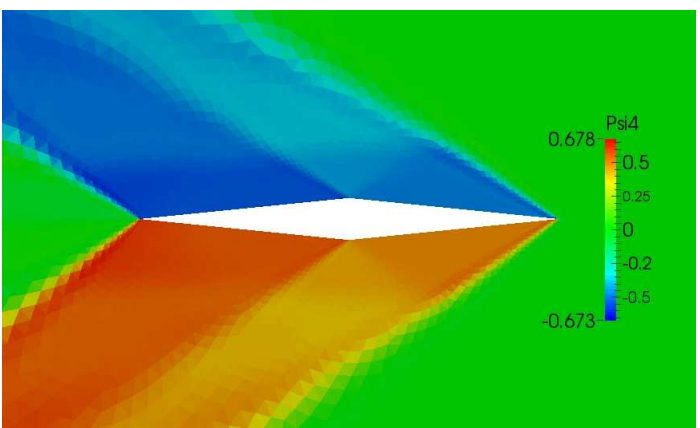

(d) $\varphi_{4}$

Figura 4.12: Solução adjunta de um esoamento supersônico externo em perfil diamante com número de Mach de escoamento livre, $M_{\infty}=1.8$, e ângulo de ataque, $\theta=2^{\circ}$.

As figuras 4.13 - 4.17, apresentadas a seguir, exibem a comparação das sensibilidades da força de sustentação obtidas pelos métodos adjunto e de diferenças finitas, com relação às variáveis primitivas na fronteira de entrada escoamento no domínio no perfil diamante descrito com ângulos de ataque que variam de $0^{\circ}$ à $4^{\circ}$.

Claramente, ambos os gradientes, adjunto e força bruta, convergem para valores fixos (para cada ângulo de ataque) em todas as condições supersônicas testadas a medida em que se avança com o número de Mach. Mesmo com uma malha relativamente "grossa" o método parece lidar bem com ondas de choque. 


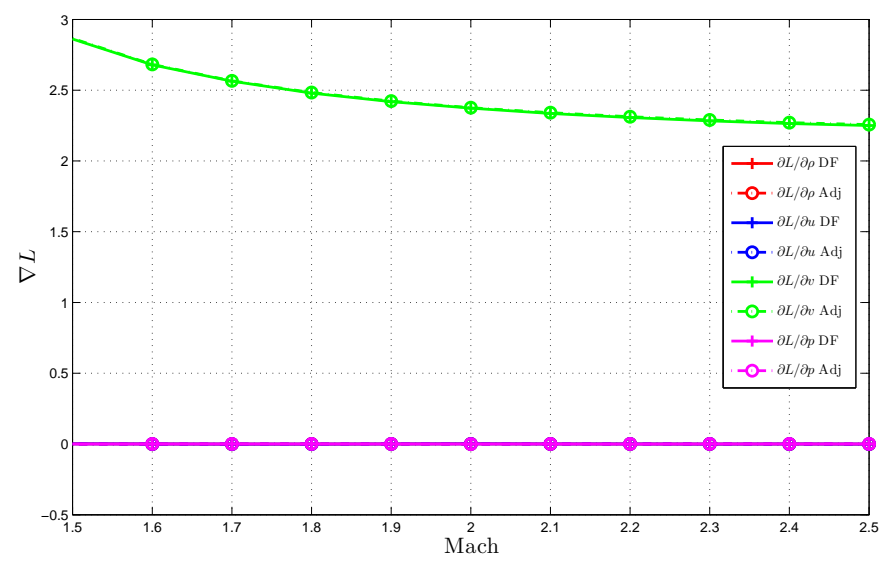

Figura 4.13: Sensibilidade da sustentação em perfil diamante com $\theta=0^{\circ}$. Linhas sólidas com marcador ' + ', diferenças finitas; traço-ponto com marcador 'o', adjunto. Vermelho, $\partial L / \partial \rho$; azul, $\partial L / \partial u$; verde, $\partial L / \partial v$; magenta, $\partial L / \partial p$.

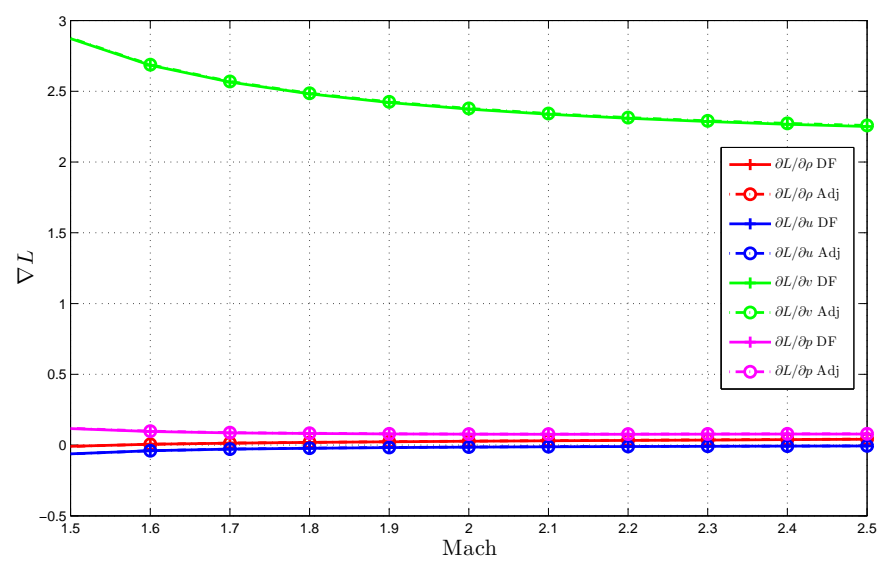

Figura 4.14: Sensibilidade da sustentação em perfil diamante com $\theta=1^{\circ}$. Linhas sólidas com marcador ' + ', diferenças finitas; traço-ponto com marcador 'o', adjunto. Vermelho, $\partial L / \partial \rho$; azul, $\partial L / \partial u$; verde, $\partial L / \partial v$; magenta, $\partial L / \partial p$.

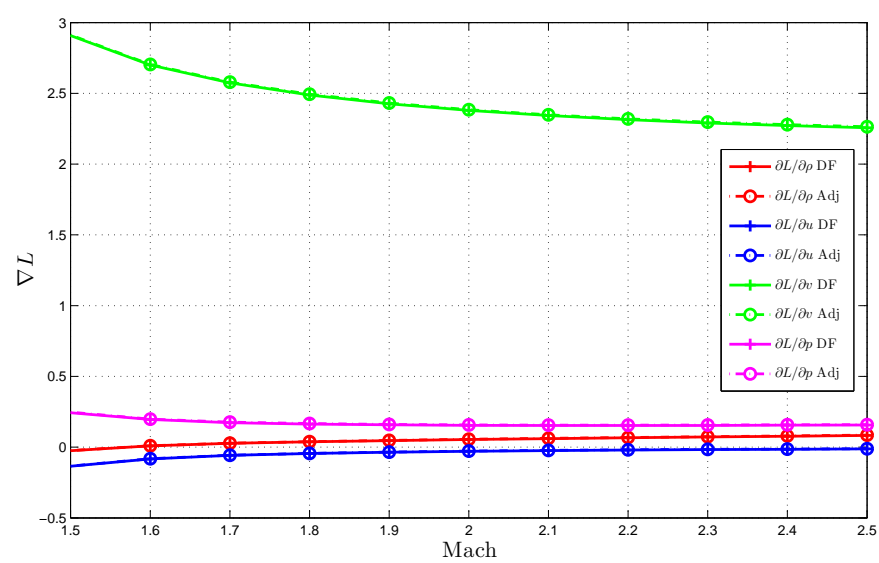

Figura 4.15: Sensibilidade da sustentação em perfil diamante com $\theta=2^{\circ}$. Linhas sólidas com marcador ' + ', diferenças finitas; traço-ponto com marcador 'o', adjunto. Vermelho, $\partial L / \partial \rho$; azul, $\partial L / \partial u$; verde, $\partial L / \partial v$; magenta, $\partial L / \partial p$. 


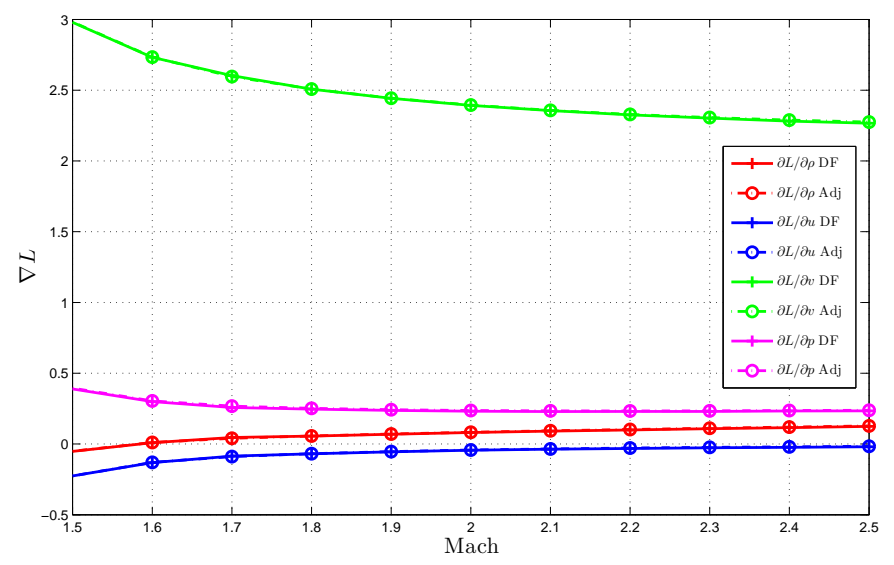

Figura 4.16: Sensibilidade da sustentação em perfil diamante com $\theta=3^{\circ}$. Linhas sólidas com marcador ' + ', diferenças finitas; traço-ponto com marcador 'o', adjunto. Vermelho, $\partial L / \partial \rho$; azul, $\partial L / \partial u$; verde, $\partial L / \partial v$; magenta, $\partial L / \partial p$.

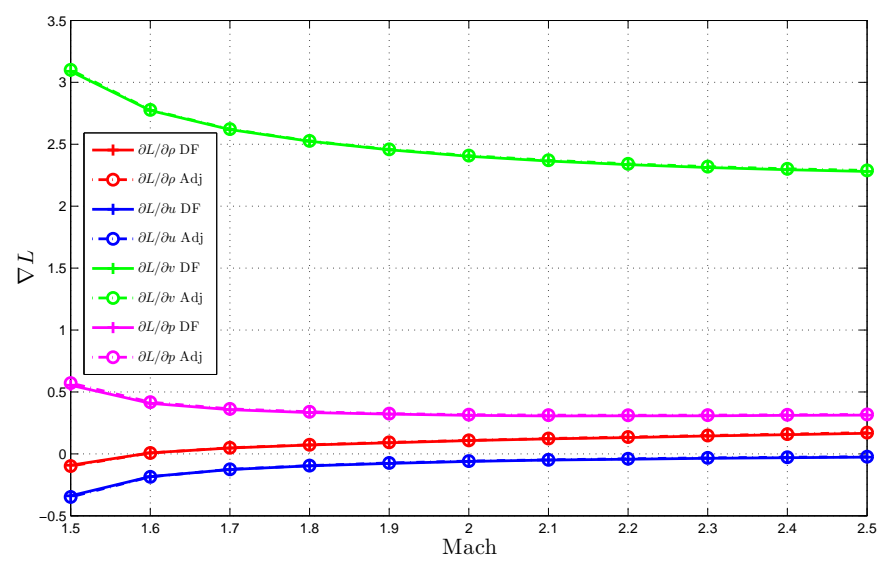

Figura 4.17: Sensibilidade da sustentação em perfil diamante com $\theta=4^{\circ}$. Linhas sólidas com marcador ' + ', diferenças finitas; traço-ponto com marcador 'o', adjunto. Vermelho, $\partial L / \partial \rho$; azul, $\partial L / \partial u$; verde, $\partial L / \partial v$; magenta, $\partial L / \partial p$.

A figura 4.18 mostra os históricos de convergência dos problemas físico e adjunto para o caso em que o número de Mach de escoamento livre é $M_{\infty}=1.8$ e o ângulo de ataque é $\theta=1^{\circ}$.

De posse das soluções encontradas para essas situações, é possível uni-las, gerando superfícies para cada uma das derivadas computadas. As figuras 4.19, 4.20, 4.21 e 4.22 representam exatamente essa construção para cada um dos quatro componentes do gradiente adjunto para ângulos de ataque variando de $0^{\circ}$ à $4^{\circ} \mathrm{e}$ números de Mach na faixa de 1.5 à 2.5.

Observa-se que o gradiente $\partial L / \partial v$ é o único não-nulo no caso particular em que o ângulo de ataque $\theta=0^{\circ}$, o que é, naturalmente, esperado devido a simetria da geometria em questão. Além disso, a magnitude das demais derivadas $\partial L / \partial \rho$, $\partial L / \partial u$ e $\partial L / \partial p$ cresce a medida em que o ângulo de ataque sobe. É interessante 

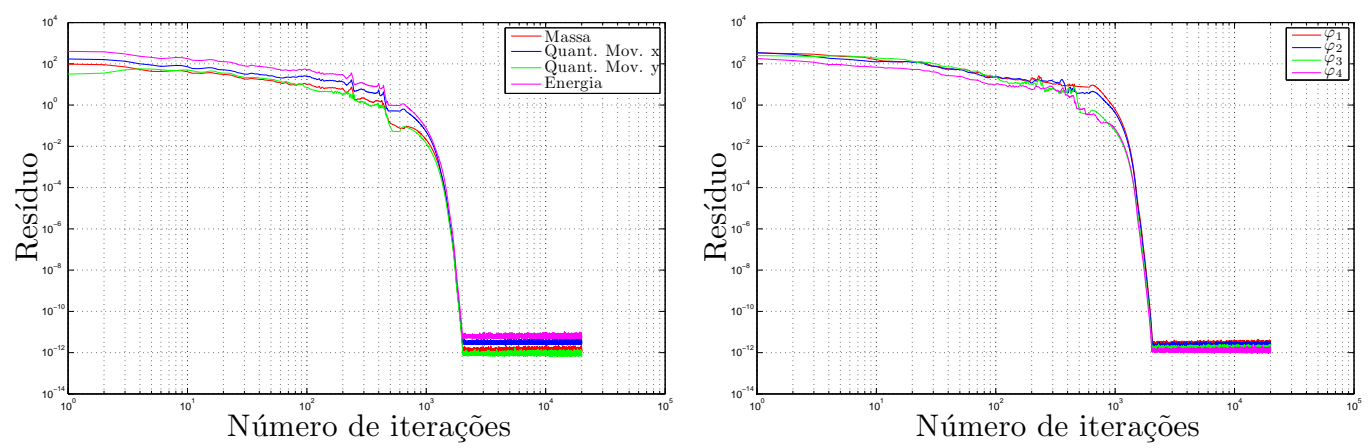

(a) Problema físico. Vermelho, massa; azul, (b) Problema adjunto. Vermelho, $\varphi_{1}$; azul, quant. mov. x; verde, quant. mov. y; ma- $\varphi_{2}$; verde, $\varphi_{3}$; magenta, $\varphi_{4}$. genta, energia.

Figura 4.18: Comparação dos históricos de convergência dos problemas físico e adjunto em perfil diamante em regime de escoamento supersônico com número de Mach de escoamento livre, $M_{\infty}=1.8$, e o ângulo de ataque, $\theta=1^{\circ}$.

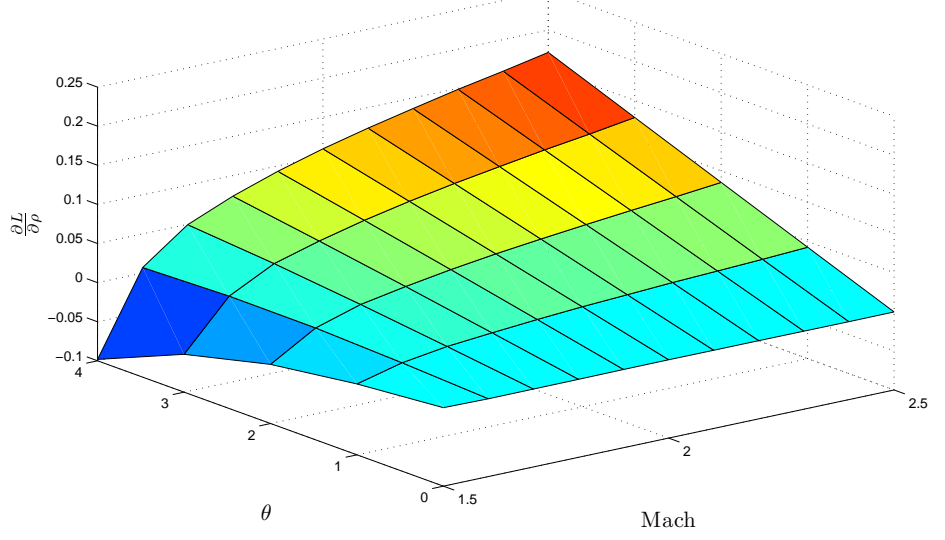

Figura 4.19: Perfil diamante $-\partial L / \partial \rho$

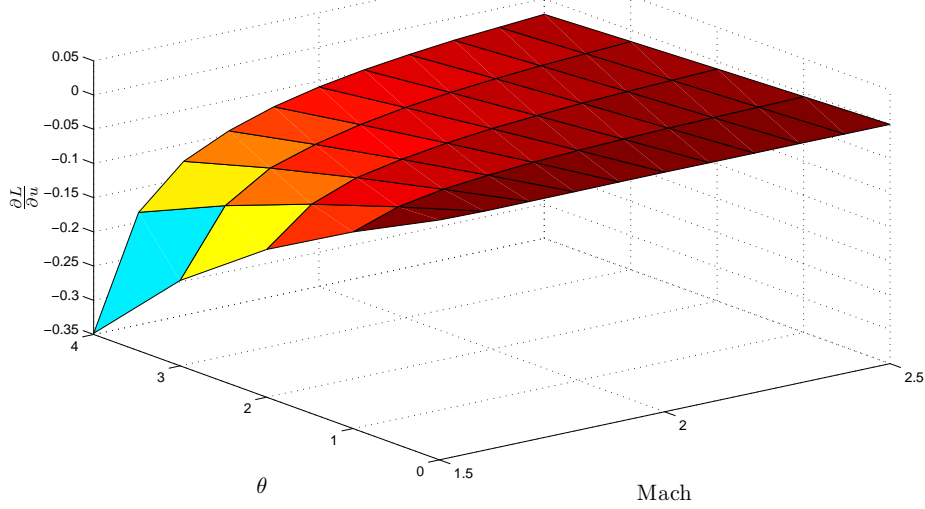

Figura 4.20: Perfil diamante $-\partial L / \partial u$

notar também o comportamento assintótico de todas elas à medida que o número de Mach é aumentado. O gradiente $\partial L / \partial u$, em particular, chama a atenção para o fato de tender a zero com o incremento de $M$, independentemente do ângulo de 


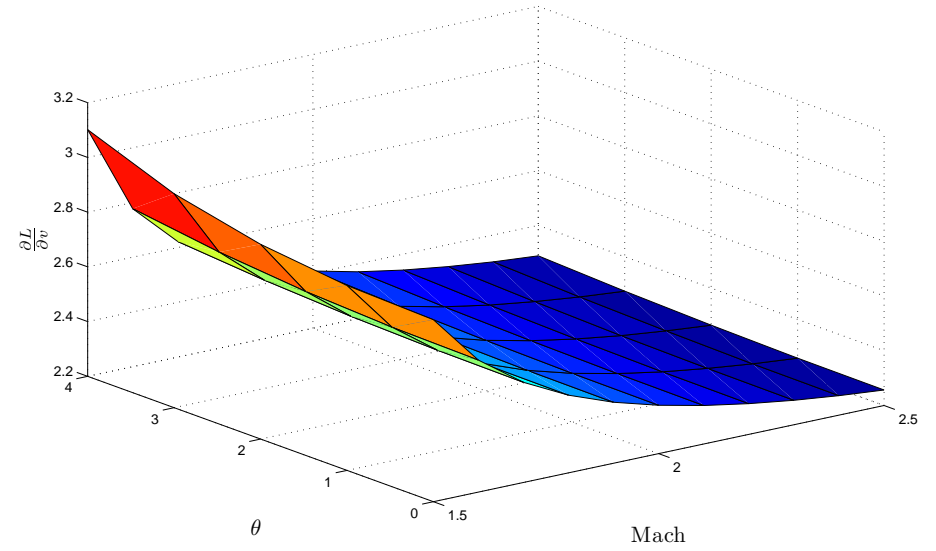

Figura 4.21: Perfil diamante $-\partial L / \partial v$

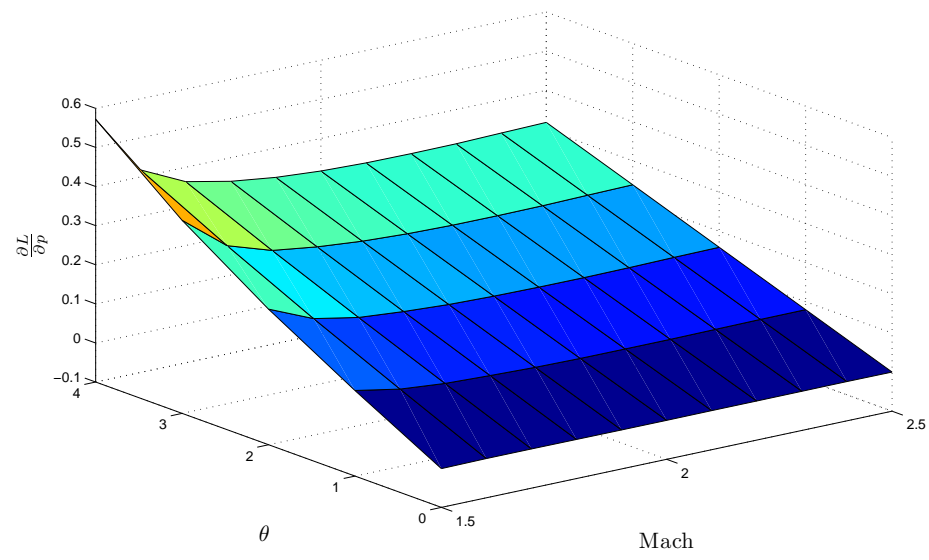

Figura 4.22: Perfil diamante $-\partial L / \partial p$

ataque. Em outras palavras, variações da componente horizontal da velocidade se tornam irrelevantes para a sustentação em altas velocidades com esse tipo de geometria.

Como já destacado, um dos grandes trunfos do método adjunto é a sua flexibilidade com relação a escolha da medida de mérito adotada. Com uma simples alteração na condição de contorno de parede é possível, por exemplo, computar a sensibilidade do arrasto de onda com relação às mesmas variáveis, como mostram as figuras $4.23-4.27$.

É claro que, assim como nos casos em que o funcional objetivo era a força de sustentação, também é possível unir os resultados da sensibilidade do arrasto de onda, gerando superfícies que descrevem as derivadas em questão sob as condições de escoamento livre computadas. As figuras 4.28 - 4.31 mostram, justamente, esses resultados para a faixa de números de Mach de 1.5 à 2.5 e ângulos de ataque de $0^{\circ}$ à $4^{\circ}$. 


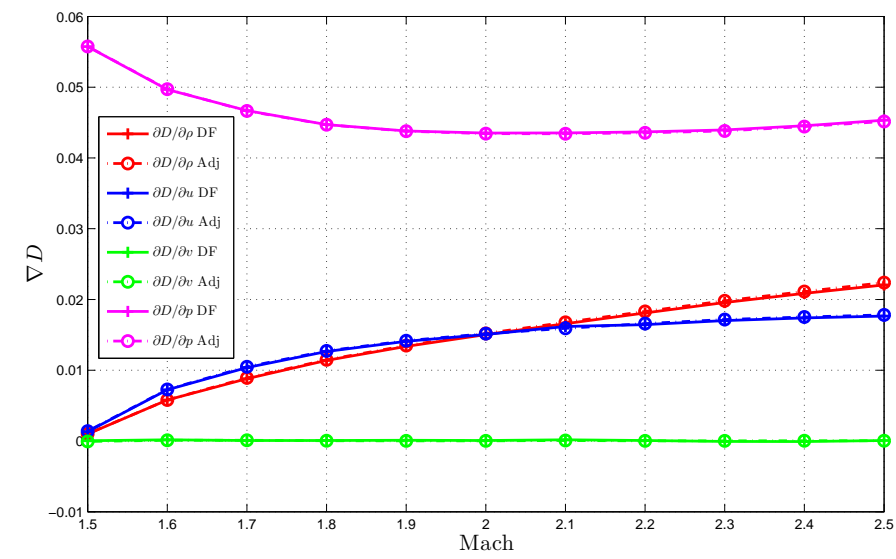

Figura 4.23: Sensibilidade do arrasto de onda em perfil diamante $\operatorname{com} \theta=0^{\circ}$. Linhas sólidas com marcador ' + ', diferenças finitas; traço-ponto com marcador 'o', adjunto. Vermelho, $\partial D / \partial \rho$; azul, $\partial D / \partial u$; verde, $\partial D / \partial v$; magenta, $\partial D / \partial p$.

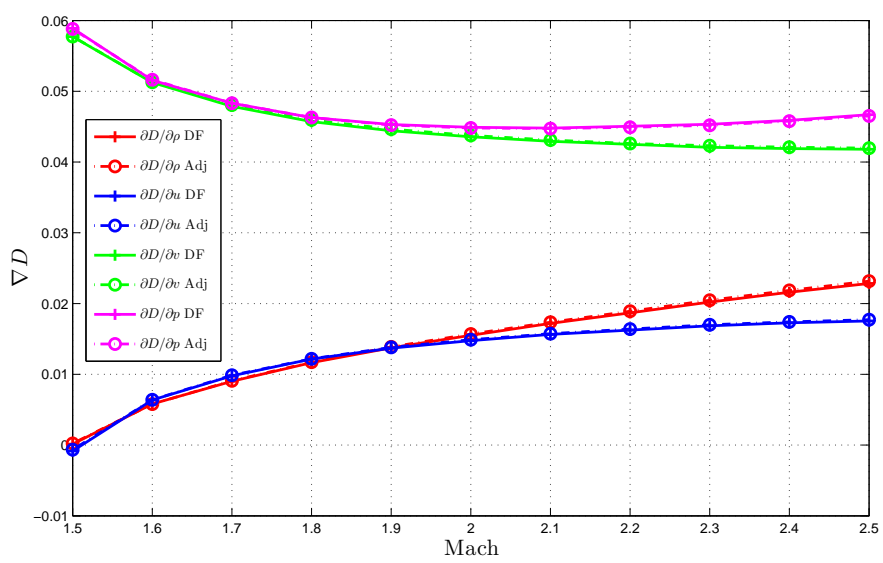

Figura 4.24: Sensibilidade do arrasto de onda em perfil diamante $\operatorname{com} \theta=1^{\circ}$. Linhas sólidas com marcador ' + ', diferenças finitas; traço-ponto com marcador 'o', adjunto. Vermelho, $\partial D / \partial \rho$; azul, $\partial D / \partial u$; verde, $\partial D / \partial v$; magenta, $\partial D / \partial p$.

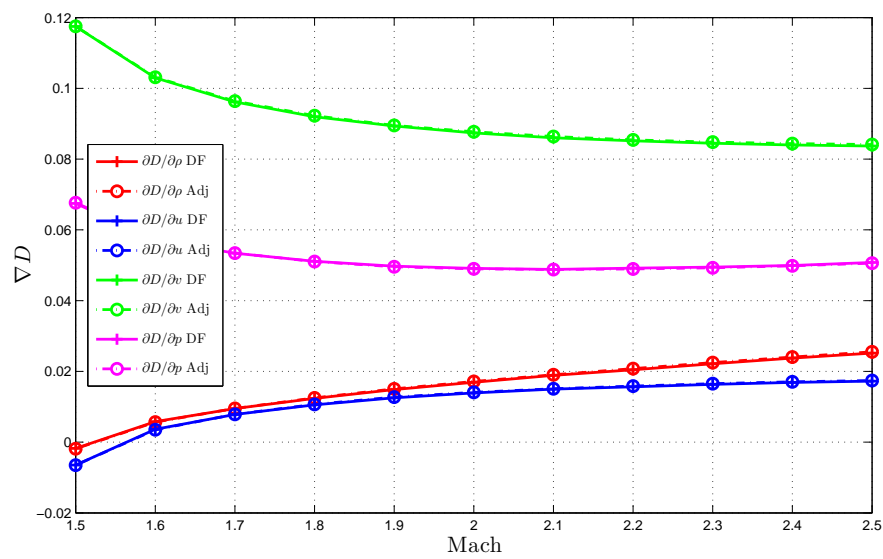

Figura 4.25: Sensibilidade do arrasto de onda em perfil diamante $\operatorname{com} \theta=2^{\circ}$. Linhas sólidas com marcador ' + ', diferenças finitas; traço-ponto com marcador 'o', adjunto. Vermelho, $\partial D / \partial \rho$; azul, $\partial D / \partial u$; verde, $\partial D / \partial v$; magenta, $\partial D / \partial p$. 


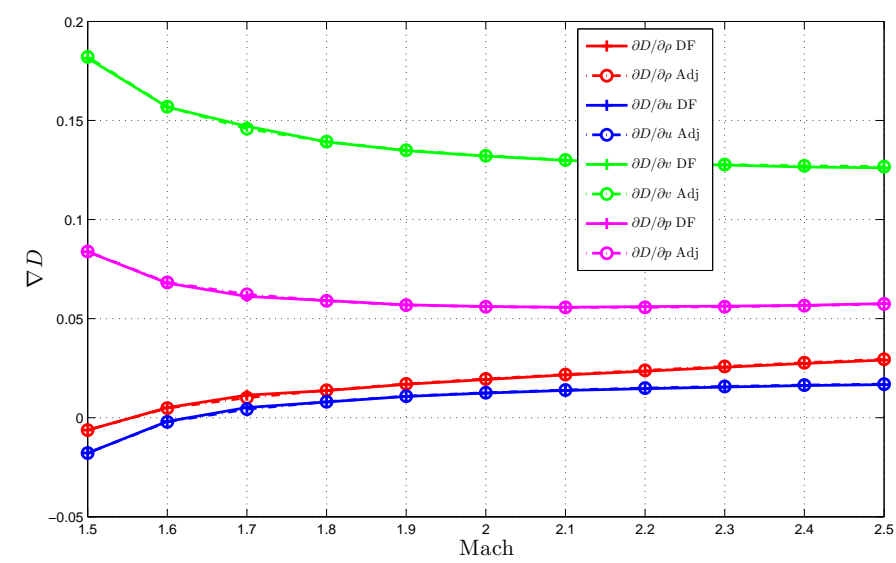

Figura 4.26: Sensibilidade do arrasto de onda em perfil diamante $\operatorname{com} \theta=3^{\circ}$. Linhas sólidas com marcador ' + ', diferenças finitas; traço-ponto com marcador 'o', adjunto. Vermelho, $\partial D / \partial \rho$; azul, $\partial D / \partial u$; verde, $\partial D / \partial v$; magenta, $\partial D / \partial p$.

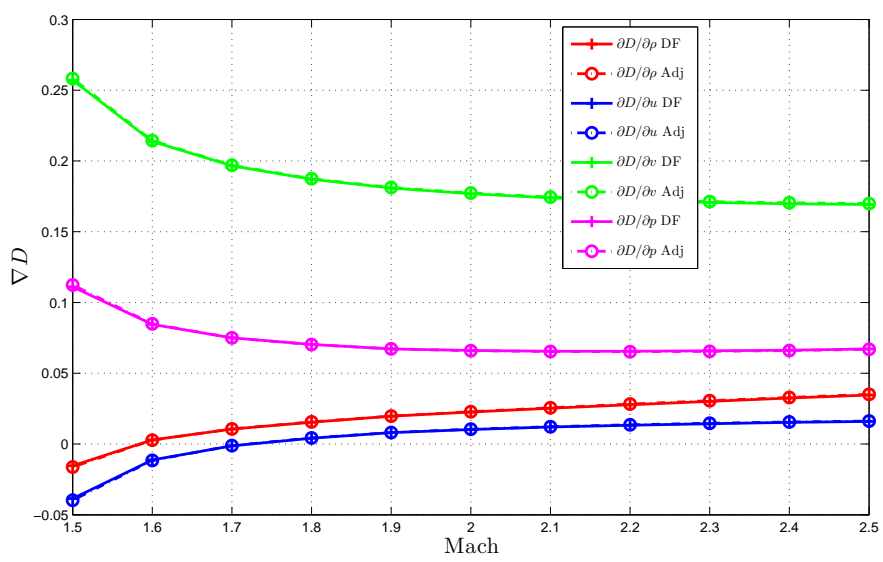

Figura 4.27: Sensibilidade do arrasto de onda em perfil diamante com $\theta=4^{\circ}$. Linhas sólidas com marcador ' + ', diferenças finitas; traço-ponto com marcador 'o', adjunto. Vermelho, $\partial D / \partial \rho$; azul, $\partial D / \partial u$; verde, $\partial D / \partial v$; magenta, $\partial D / \partial p$.

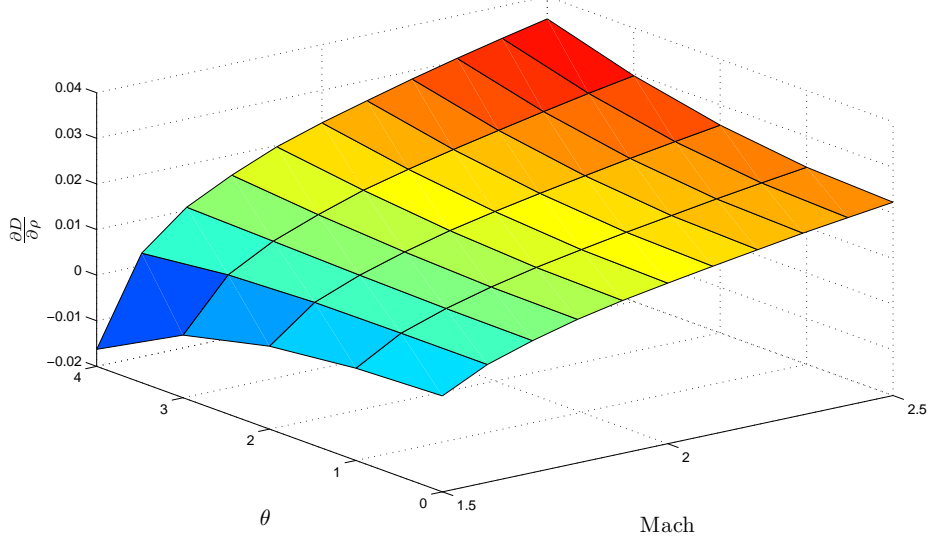

Figura 4.28: Perfil diamante $-\partial D / \partial \rho$

Os resultados até aqui apresentados demonstram que a abordagem do problema adjunto proposta neste trabalho é capaz de lidar com escoamentos nos 


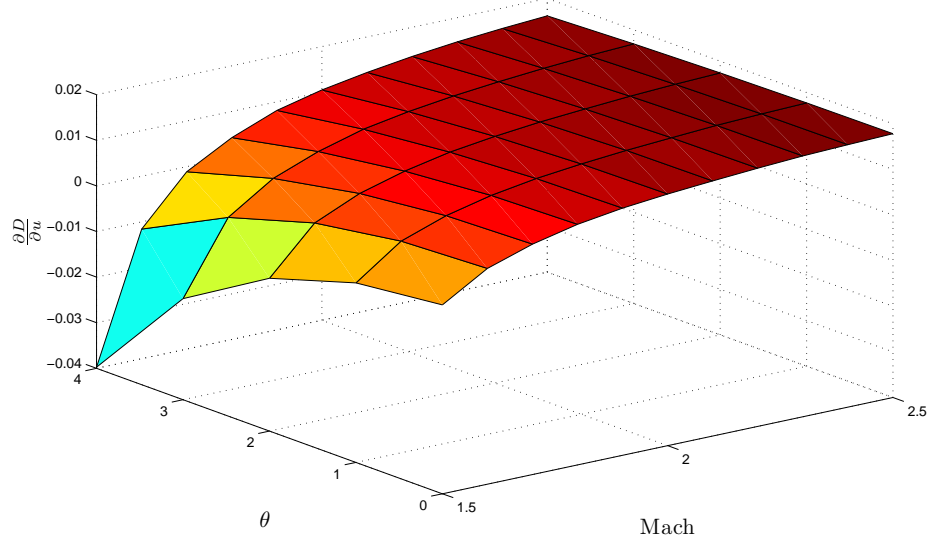

Figura 4.29: Perfil diamante $-\partial D / \partial u$

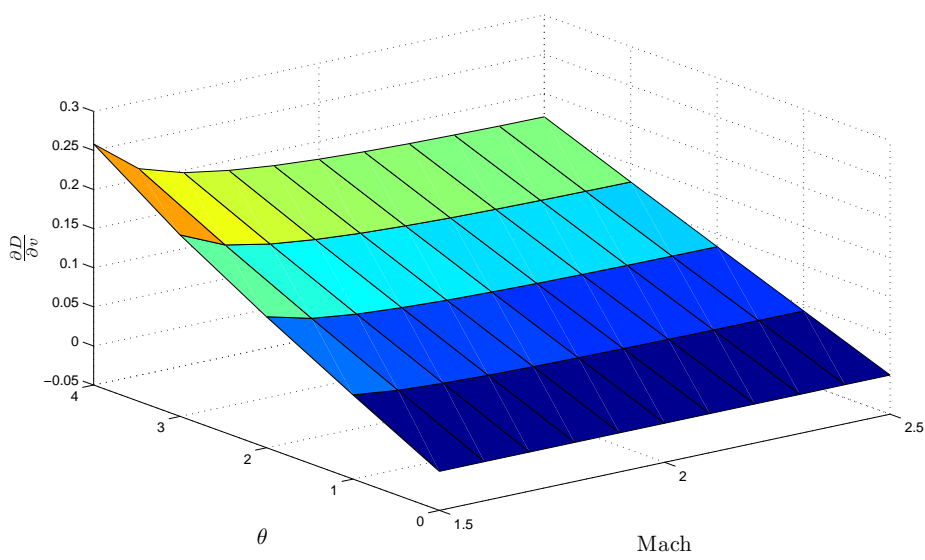

Figura 4.30: Perfil diamante $-\partial D / \partial v$

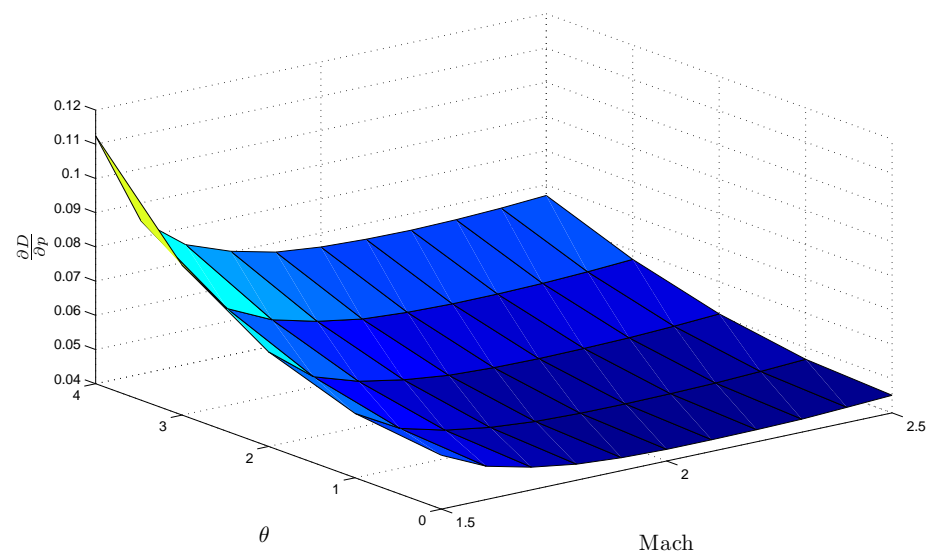

Figura 4.31: Perfil diamante $-\partial D / \partial p$

regimes de escoamento subsônico e supersônico com e sem a presença de ondas de choque, fornecendo gradientes bastante condizentes com os obtidos por diferenças finitas, porém com custo computacional bem menor. Entretanto, estes testes não esgotam as possibilidades que a física proporciona. A literatura mostra que, tipicamente, escoamentos em regime transônico são os que causam as maio- 
res dificuldades nas simulações de CFD. Prosseguindo com a ordem cresecente de complexidade de aplicações, as próximas duas seções tratam, exatamente, desse regime de escoamento.

\subsubsection{Perfil RAE 2822}

Nesta e na próxima seção, serão finalmente apresentados os casos de maior interesse para a indústria aeroespacial: os escoamentos transônicos. Todas as simulações incluídas até o fim deste capítulo correspondem a um dos casos listados na tabela 4.2 como condições de contorno impostas em fronteiras permeáveis.

Tabela 4.2: Condições de contorno impostas em escoamentos subsôni$\cos$ /transônicos externos (propriedades adimensionais)

\begin{tabular}{|c|c|c|c|c|}
\hline \multicolumn{4}{|c|}{ Entrada } & Saída \\
\hline Caso & $p_{o}$ & $T_{o}$ & $\theta$ & $p$ \\
\hline 1 & 0.847295 & 0.75 & $0^{\circ}$ a $4^{\circ}$ & 0.714286 \\
\hline 2 & 0.911074 & 0.765714 & $0^{\circ}$ a $4^{\circ}$ & 0.714286 \\
\hline 3 & 0.990787 & 0.784286 & $0^{\circ}$ a $4^{\circ}$ & 0.714286 \\
\hline 4 & 1.088814 & 0.805714 & $0^{\circ}$ a $4^{\circ}$ & 0.714286 \\
\hline 5 & 1.208074 & 0.83 & $0^{\circ}$ a $4^{\circ}$ & 0.714286 \\
\hline
\end{tabular}

Esta seção, em particular, trata das simulações feitas em torno do aerofólio RAE 2822. Os casos 1 a 5 descritos na tabela 4.2 correspondem às soluções estacionárias do escoamento em que o número de Mach é: 0.5, 0.6, 0.7, 0.8 e 0.9, respectivamente, nas fronteiras de entrada.

Seguindo a mesma sequencia dos testes anteriores, considere, também, a malha computacional utilizada e uma solução deste aerofólio na situação com $M=0.9$ e $\theta=0^{\circ}$, apresentada na fig. 4.32 afim de elucidar o tipo de escoamento agora analisado.

A fig. 4.33, por sua vez, apresenta os contornos das 4 variáveis adjuntas obtidas a partir da solução da fig. 4.32 .

A comparação da sensibilidade da força de sustentação exercida sobre o aerofólio RAE 2822 determinada pelos métodos adjunto e diferenças finitas é finalmente exibida nas figuras 4.34 - 4.38 para escoamentos com ângulo de ataque entre $0^{\circ}$ e $4^{\circ}$. Assim como nos demais casos subsônicos apresentados neste trabalho, $\partial L / \partial p_{o}, \partial L / \partial T_{o}$, e $\partial L / \partial \theta$ são as sensibilidades avaliadas.

Nitidamente, há uma piora na qualidade dos resultados presentes nesta seção 


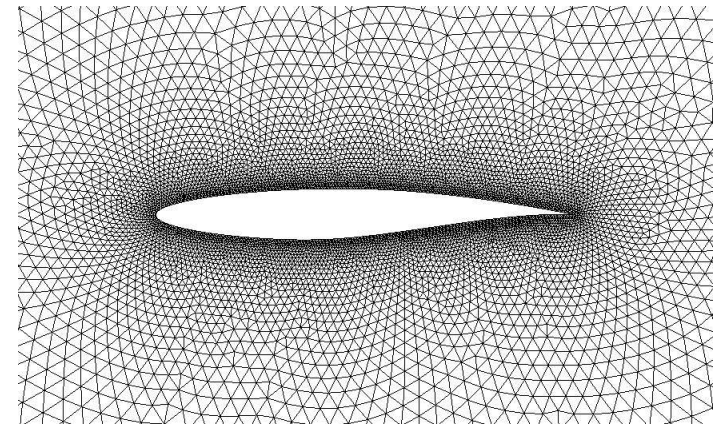

(a) Malha

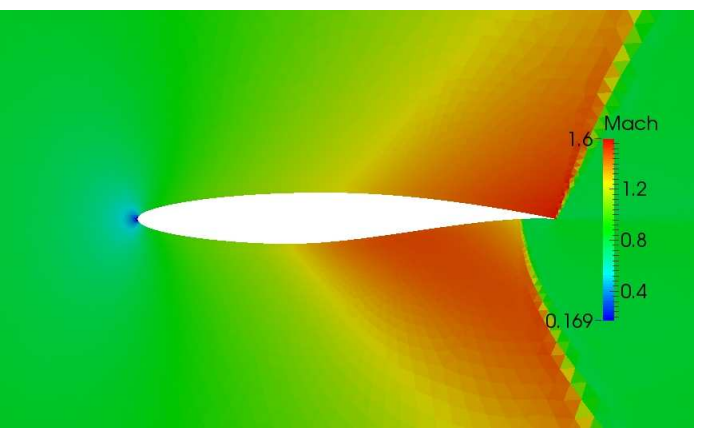

(b) Contorno de Mach

Figura 4.32: Solução do perfil RAE 2822 com número de Mach de escoamento livre, $M_{\infty}=0.9$, e ângulo de ataque, $\theta=0^{\circ}$.

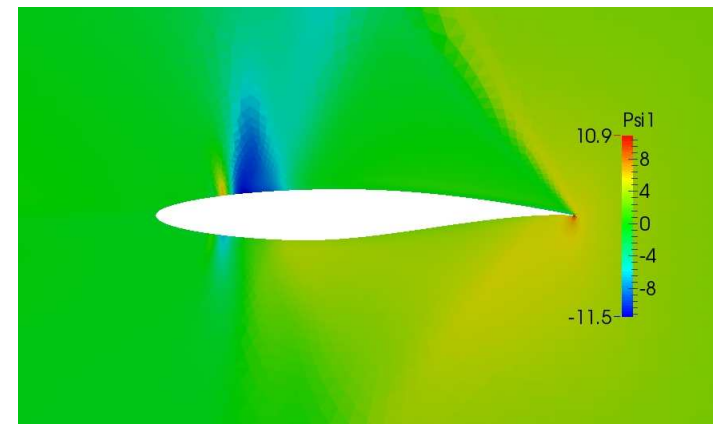

(a) $\varphi_{1}$

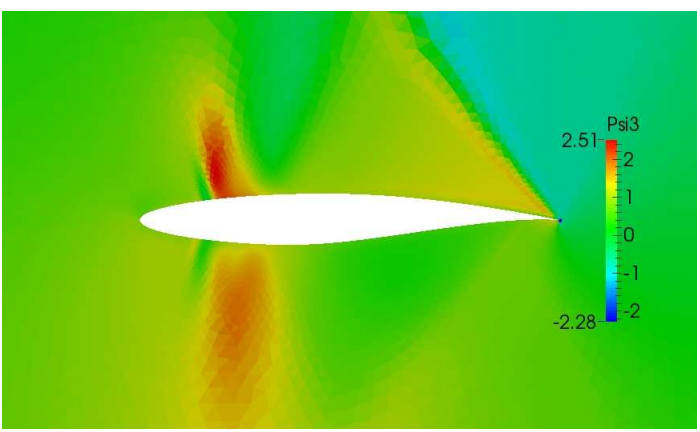

(c) $\varphi_{3}$

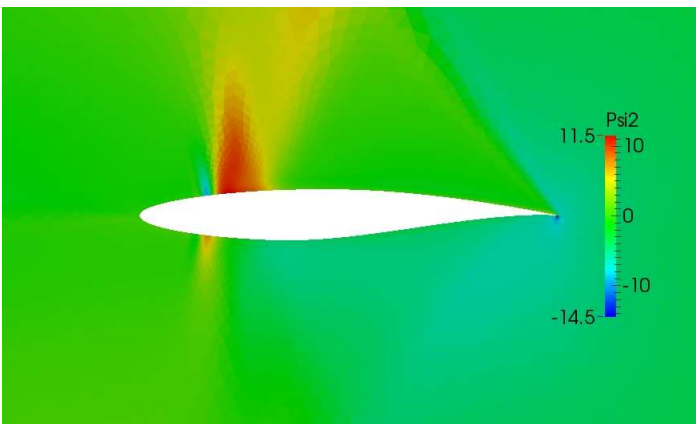

(b) $\varphi_{2}$

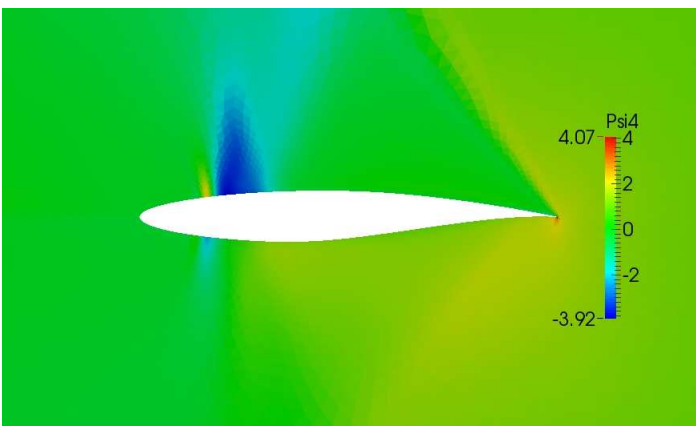

(d) $\varphi_{4}$

Figura 4.33: Solução adjunta de um esoamento subsônico externo em perfil RAE 2822 com número de Mach de escoamento livre, $M_{\infty}=0.9$, e ângulo de ataque, $\theta=0^{\circ}$.

ao compará-los com os casos anteriores. Os piores resultados ocorrem, particularmente, no regime de escoamento transônico, como o caso em que $M=0.8$ e $\theta=3^{\circ}$ exibido na fig. 4.37 que, mesmo com algum refinamento de malha não teve o seu nível de precisão como nas geometrias anteriormente testadas. Ainda assim, os demais testes parecem exibir gradientes bastante condizentes. É interessante notar que há situações transônicas com presença de choque com boas aproximações e enquanto outras não. A princípio, uma boa alternativa para tentar melhorar esses resultados seria através da sistematização de um estudo de malhas, propondo uma sequencia progressivamente mais refinada, analisando 


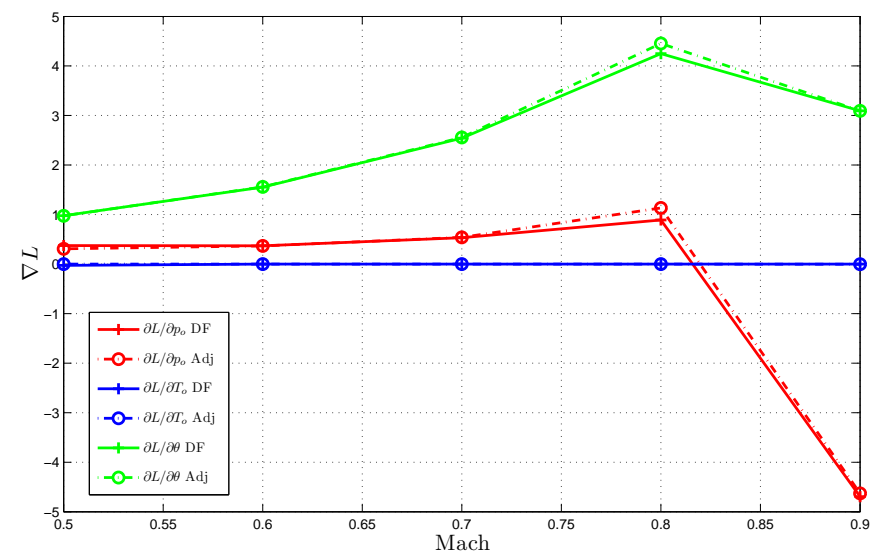

Figura 4.34: Sensibilidade da sustentação em perfil RAE $2822 \operatorname{com} \theta=0^{\circ}$. Linhas sólidas com marcador ' + ', diferenças finitas; traço-ponto com marcador 'o', adjunto. Vermelho, $\partial L / \partial p_{o}$; azul, $\partial L / \partial T_{o}$; verde, $\partial L / \partial \theta$.

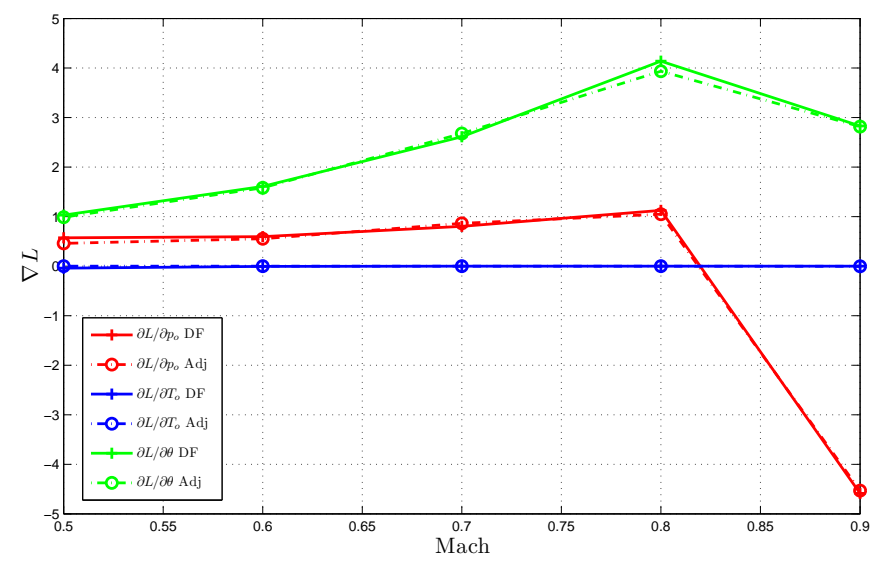

Figura 4.35: Sensibilidade da sustentação em perfil RAE $2822 \operatorname{com} \theta=1^{\circ}$. Linhas sólidas com marcador ' + ', diferenças finitas; traço-ponto com marcador 'o', adjunto. Vermelho, $\partial L / \partial p_{o}$; azul, $\partial L / \partial T_{o}$; verde, $\partial L / \partial \theta$.

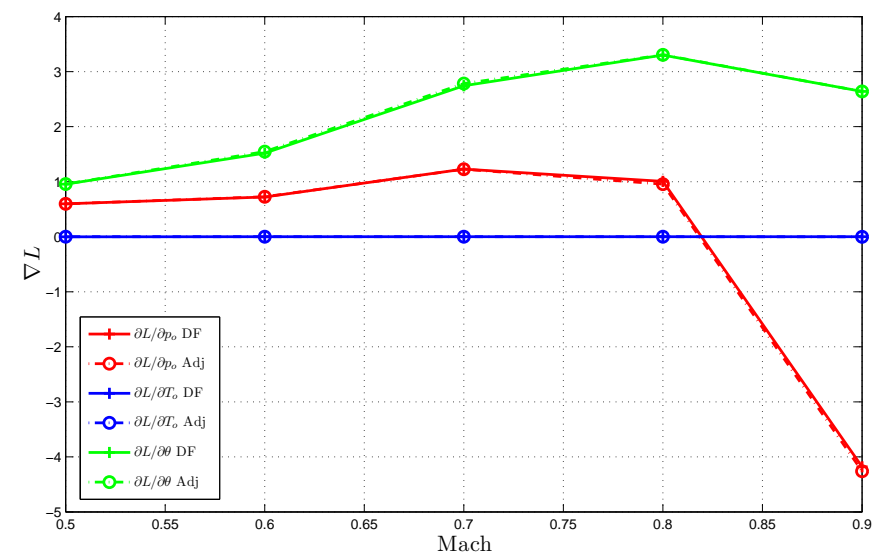

Figura 4.36: Sensibilidade da sustentação em perfil RAE $2822 \operatorname{com} \theta=2^{\circ}$. Linhas sólidas com marcador ' + ', diferenças finitas; traço-ponto com marcador 'o', adjunto. Vermelho, $\partial L / \partial p_{o}$; azul, $\partial L / \partial T_{o}$; verde, $\partial L / \partial \theta$. 


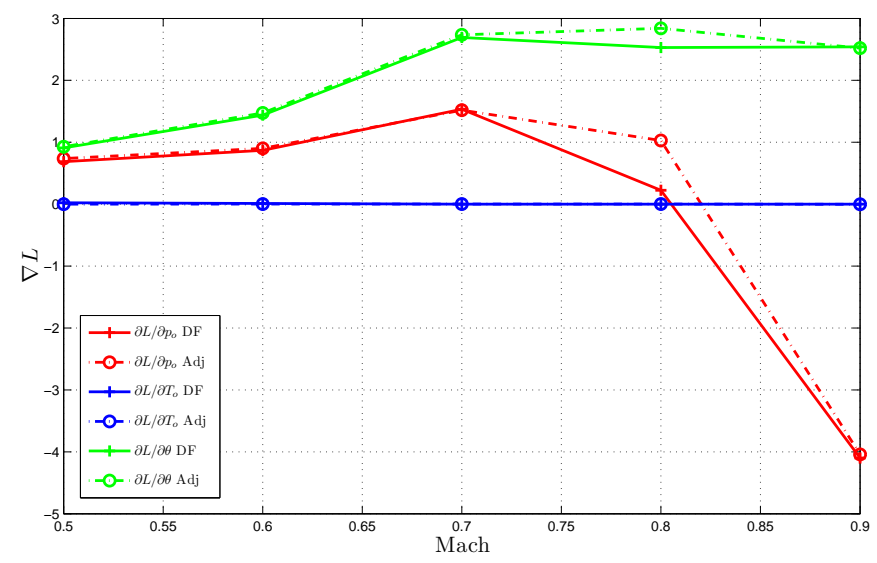

Figura 4.37: Sensibilidade da sustentação em perfil RAE $2822 \operatorname{com} \theta=3^{\circ}$. Linhas sólidas com marcador ' + ', diferenças finitas; traço-ponto com marcador 'o', adjunto. Vermelho, $\partial L / \partial p_{o}$; azul, $\partial L / \partial T_{o}$; verde, $\partial L / \partial \theta$.

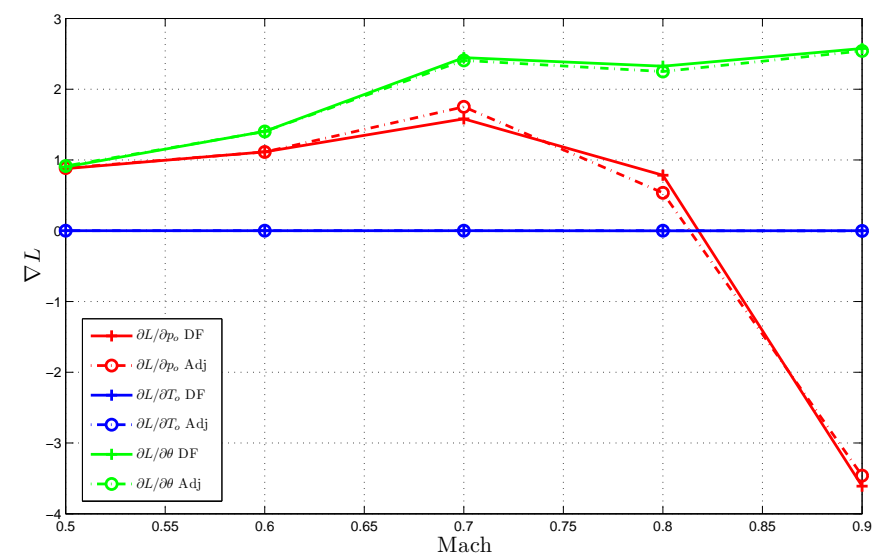

Figura 4.38: Sensibilidade da sustentação em perfil RAE $2822 \operatorname{com} \theta=4^{\circ}$. Linhas sólidas com marcador ' + ', diferenças finitas; traço-ponto com marcador 'o', adjunto. Vermelho, $\partial L / \partial p_{o}$; azul, $\partial L / \partial T_{o}$; verde, $\partial L / \partial \theta$.

então o comportamento dos gradientes. Os atributos de cada uma das malhas geradas com essa finalidade são apresentados na tabela 4.3.

É claro que esta sequencia de malhas não esgota tal estudo de refinamento. Para isso seriam necessárias malhas ainda mais refinadas. De qualquer forma, uma análise, ainda que preliminar, pode indicar as razões de resultados não tão bons quanto os apresentados até aqui. A figura 4.39 revela a todas as malhas da tabela 4.3 nas proximidades do aerofólio.

A situação que apresentou os piores resultados nas análises no RAE 2822 foi, certamente, na condição $M_{\infty}=0.8$ e $\theta=3^{\circ}$, exposta na fig. 4.41. De modo a tentar tirar maiores conclusões a respeito do ocorrido, considere os gradientes $\partial L / \partial p_{o}$ obtidos para ângulos de ataque de $\theta=3^{\circ}$ em todas as 4 malhas dessa geometria exibidas na fig. 4.40 . 
Tabela 4.3: Malhas RAE 2822

\begin{tabular}{ccc}
\hline \hline Malha & No $^{\circ}$ de elementos & No $^{\mathrm{o}}$ de elementos no aerofólio \\
\hline 1 & 11376 & 300 \\
2 & 19538 & 400 \\
3 & 31602 & 800 \\
4 & 43558 & 1200 \\
\hline \hline
\end{tabular}

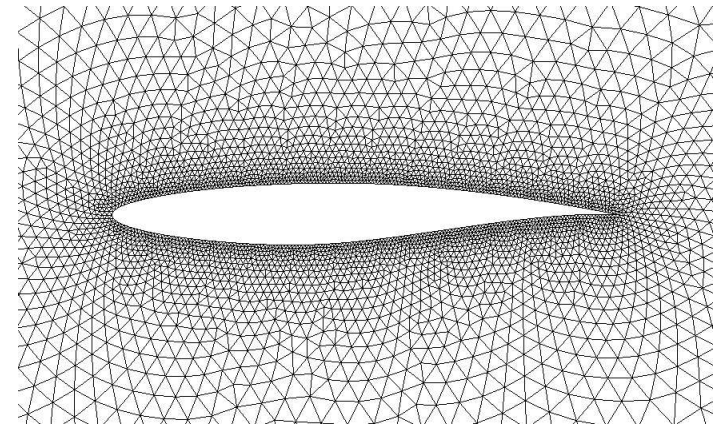

(a) Malha 1

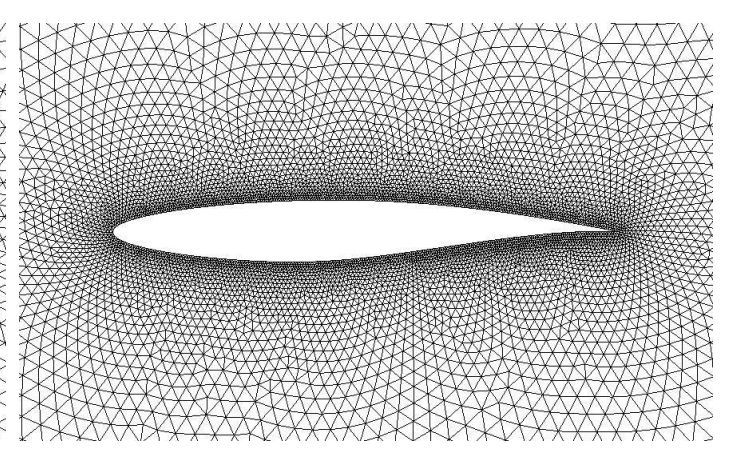

(b) Malha 2

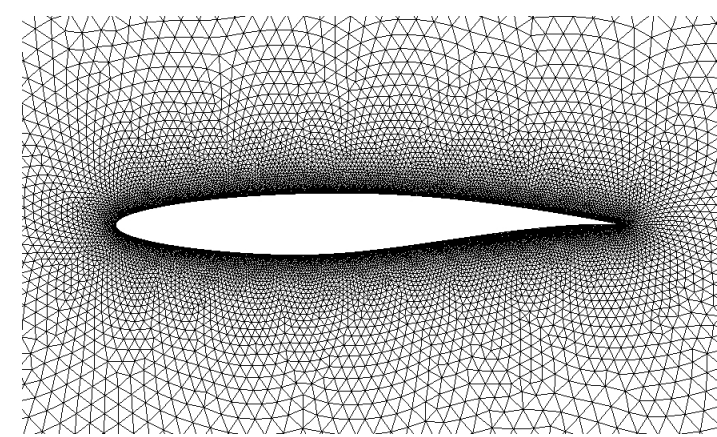

(c) Malha 3

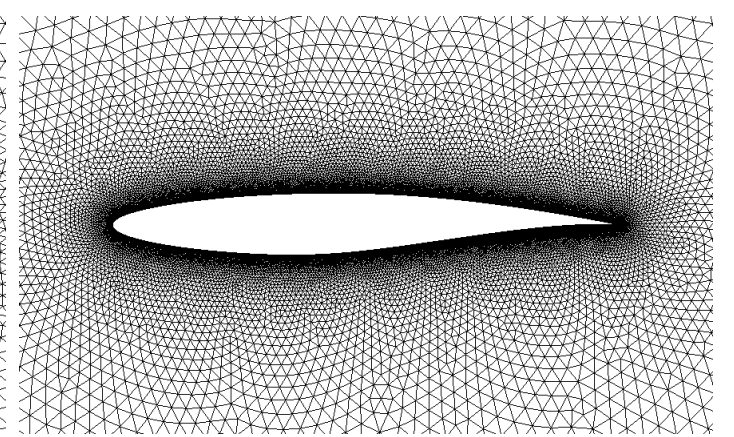

(d) Malha 4

Figura 4.39: Malhas RAE 2822

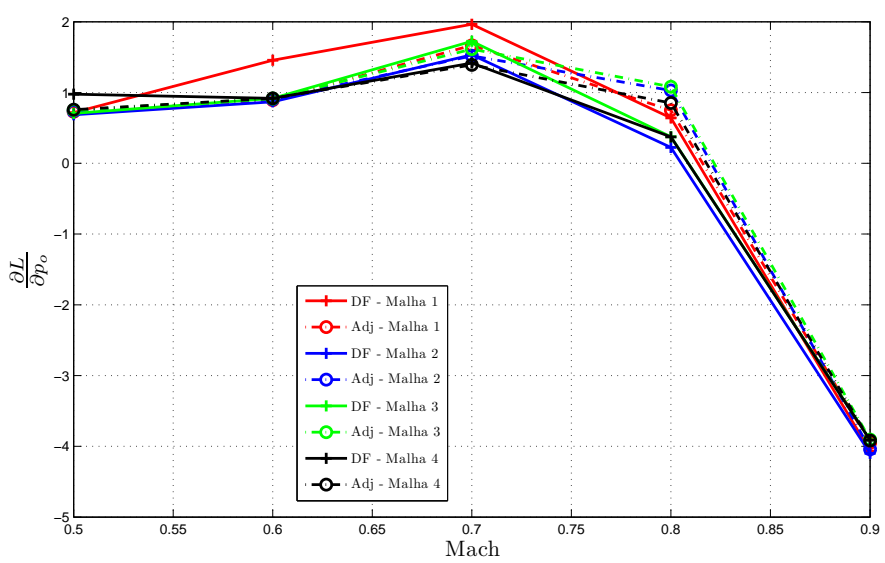

Figura 4.40: Comparação do gradiente de sensibilidade $\partial L / \partial p_{o}$ em diferentes malhas do perfil RAE $2822 \operatorname{com} \theta=3^{\circ}$. Linhas sólidas com marcador ' + ', diferenças finitas; traço-ponto com marcador 'o', adjunto. Vermelho, malha 1; azul, malha 2; verde, malha 3; preto, malha 4. 
Com exceção da malha mais "grossa", representada pela cor vermelha na fig. 4.40 que apresenta resultados, efetivamente, mais distantes dos demais. Observase que todos os gradientes estão em concordância com as sensibilidades de diferenças finitas e, convergindo para um mesmo valor a menos da condição $M=0.8$. Analisando, então, as soluções físicas desse caso expostas na fig. 4.41, é possível perceber que o posicionamento do choque no extradorso do aerofólio é ligeiramente diferente em cada uma das malhas (a régua inserida na figura ajuda a perceber o exato posicionamento do choque em cada caso).

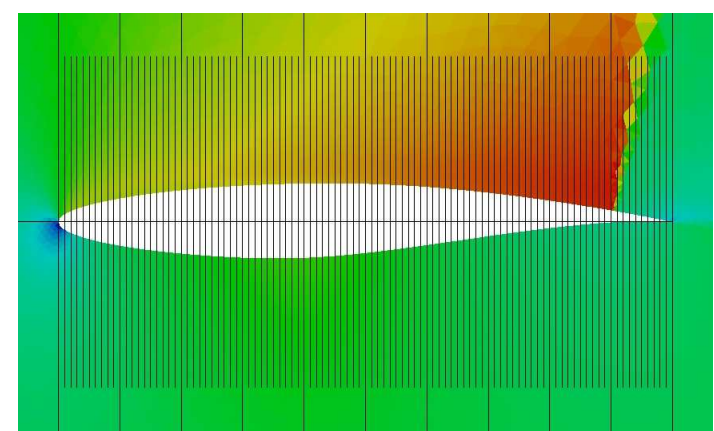

(a) Malha 1

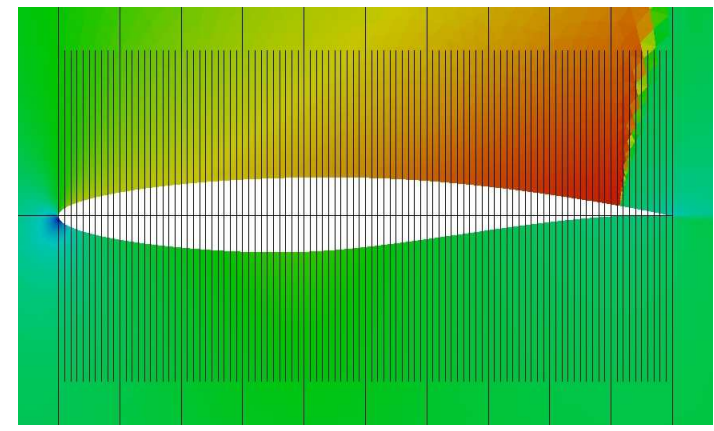

(c) Malha 3

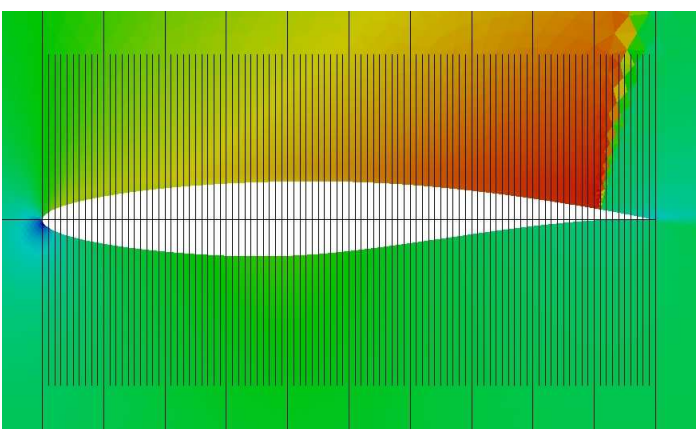

(b) Malha 2

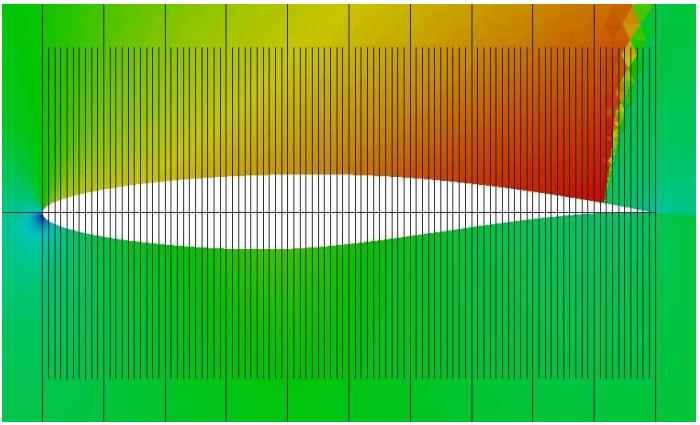

(d) Malha 4

Figura 4.41: Contornos de número de Mach do perfil RAE 2822 com número de Mach de escoamento livre, $M_{\infty}=0.8$ e ângulo de ataque, $\theta=3^{\circ}$.

O leitor poderia então se perguntar se estas soluções realmente representam estados estacionários. A resposta para essa pergunta pode ser encontrada na fig. 4.42, que apresenta o histórico de convergência das soluções da fig. 4.41. A queda do resíduo até níveis próximos de "zero máquina" indicam que cada uma delas atingiu seu estado estacionário sob as condições prescritas.

Embora ainda não haja uma demonstração matemática que prove a existência de uma solução estacionária das equações de Euler no regime transônico ${ }^{3}$, há um fator numérico que pode influenciar no posicionamento da onda de choque em

\footnotetext{
${ }^{3}$ Existem até mesmo relatos na literatura de pesquisadores como Jameson (1991), que afirma ter encontrado soluções múltiplas em escoamentos dessa natureza, justamente em situações com número de Mach entre 0.75 e 0.8 .
} 


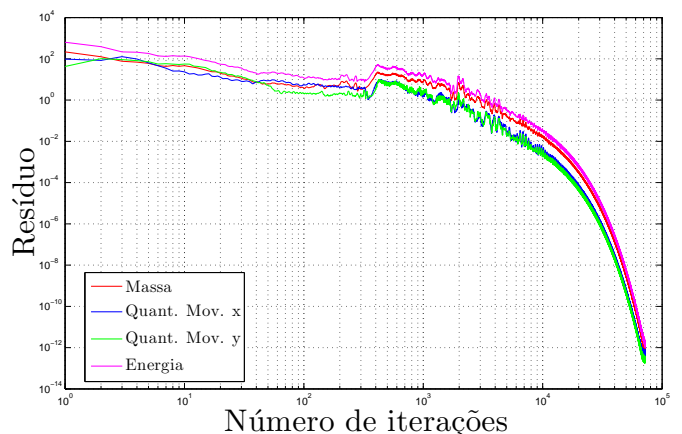

(a) Malha 1

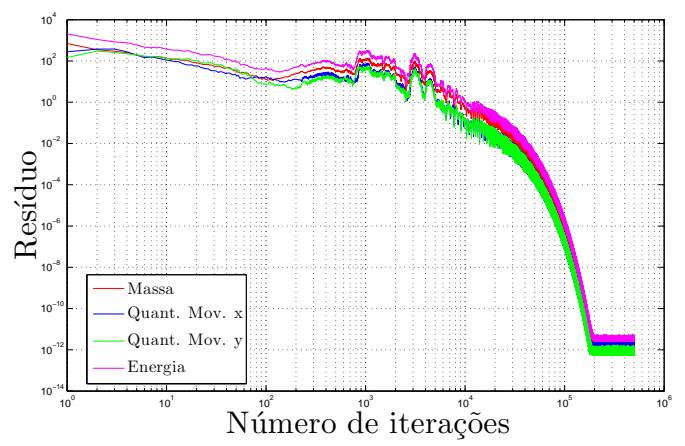

(c) Malha 3

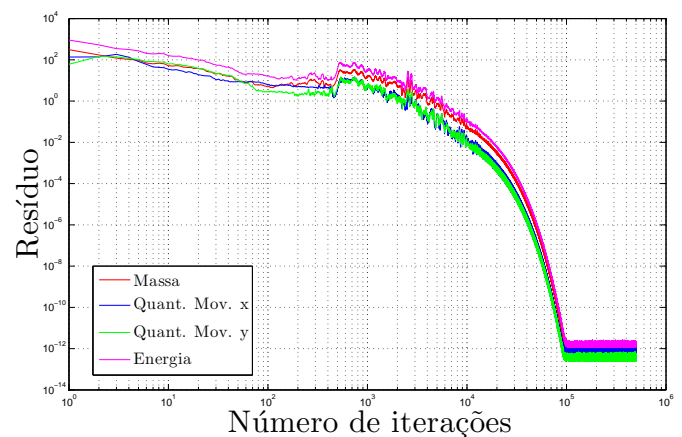

(b) Malha 2

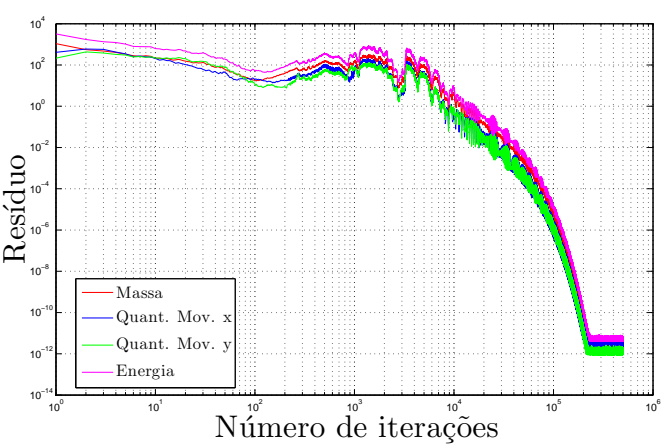

(d) Malha 4

Figura 4.42: Histórico de convergência do perfil RAE 2822 com número de Mach de escoamento livre, $M_{\infty}=0.8$, e ângulo de ataque, $\theta=3^{\circ}$.

cada uma das malhas. Para compreendê-lo, considere a variação de entropia no domínio, dada pela eq. (4.5).

$$
\Delta s=s-s_{\infty}=\frac{p}{\rho^{\gamma}}-\left(\frac{p}{\rho^{\gamma}}\right)_{\infty}
$$

onde o subscrito $\infty$ se refere às condições de escoamento ao longe.

Uma vez que a pressão de estagnação, $p_{o}$, não permanece constante ao atravessar a onda de choque, as relações invíscidas nesta interface implicam em uma variação descontínua de entropia através dele (HIRSCH, 2007). Assim, uma onda de choque, naturalmente, provocaria geração de entropia. Observe, agora, a figura 4.43, que avalia as quantidades $\Delta s$ dadas pela eq. (4.5) das soluções da figura 4.41. Note que, na região imediatamente adjacente a superfície do aerofólio, também, existe uma camada de elementos onde há geração de entropia (apesar de difícil vizualização devido à qualidade da impressão, é relativamente fácil percebê-la na fig. 4.43(a) que representa a malha mais "grossa" dentre as utilizadas para essa geometria). É claro que isso não deveria ocorrer na ausência de choque em escoamentos modelados pelas equações de Euler. Esse fato, no entanto, pode ser justificado pela dissipação artificial adicionada pelo método numérico. Uma vez que a adição de dissipação artificial se dá em maior quantidade 
nas regiões de maior gradiente de pressão, malhas mais "grossas" tem, naturalmente, mais dissipação artificial do que as malhas mais "finas". É importante notar que as soluções numéricas em todas as malhas utilizadas apresentam o que parece uma "camada limite" em diferentes graus. E é possívelmente essa diferença na quantidade de dissipação artificial que cada malha impõe a solução que esteja causando o posicionamento distinto das ondas de choque estacionárias.

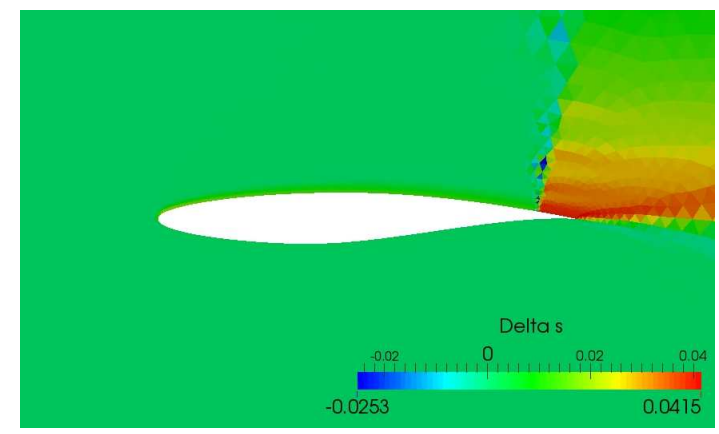

(a) Malha 1

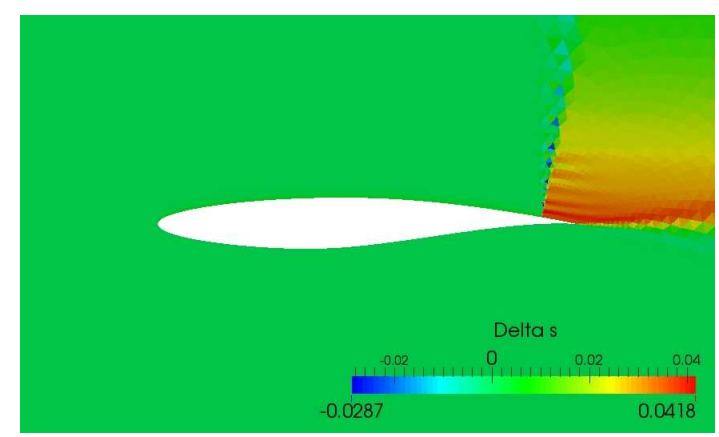

(c) Malha 3

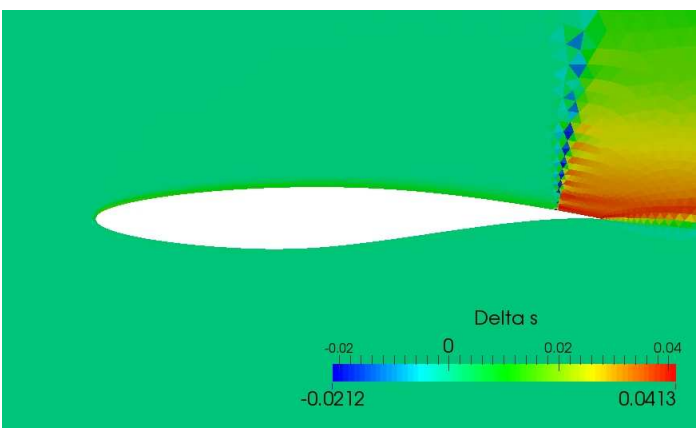

(b) Malha 2

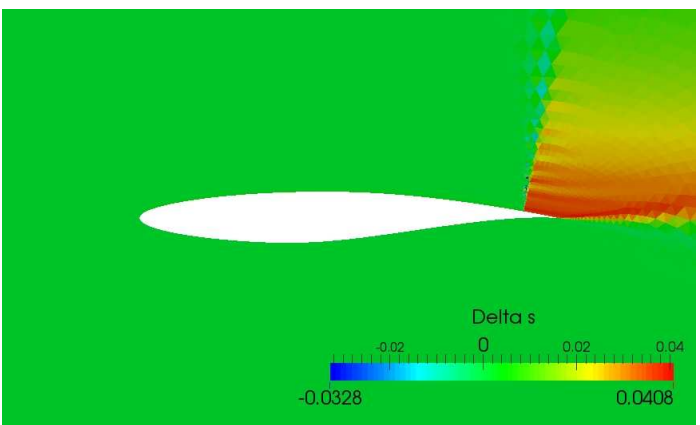

(d) Malha 4

Figura 4.43: Variação de entropia, $\Delta s$, em torno do perfil RAE 2822 em escoamento com número de Mach de escoamento livre, $M_{\infty}=0.8$, e ângulo de ataque, $\theta=3^{\circ}$.

Naturalmente, esse fato já é suficiente para explicar a diferença entre os gradientes calculados pelo método adjunto. Uma vez que eles foram obtidos a partir de soluções diferentes do escoamento, devem conduzir a diferentes sensibilidades. A ocorrência ainda leva a outras questões como, por exemplo, a precisão do gradiente computado por diferenças finitas em casos transônicos, já que as soluções perturbadas podem, igualmente, produzir soluções com choques distintos. Nesta situação a sensibilidade determinada pelo força bruta estaria "em xeque" e poderia não ser adequada para validar a solução adjunta por comparação.

O leitor pode então se perguntar se soluções com onda de choque como na situação $M=0.9$ não resultariam no mesmo problema. Ao analisar as soluções para esse caso, apresentadas na fig. 4.44 para contornos de Mach, verifica-se que em todas elas o choque mais forte está posicionado no mesmo lugar, no bordo de 
fuga do aerofólio, independentemente da malha utilizada.

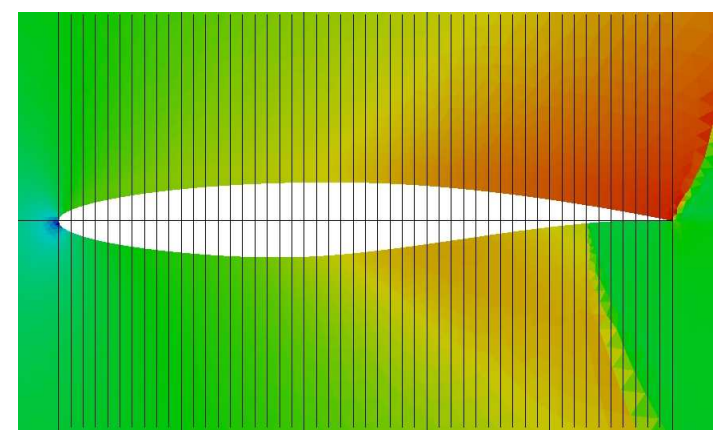

(a) Malha 1

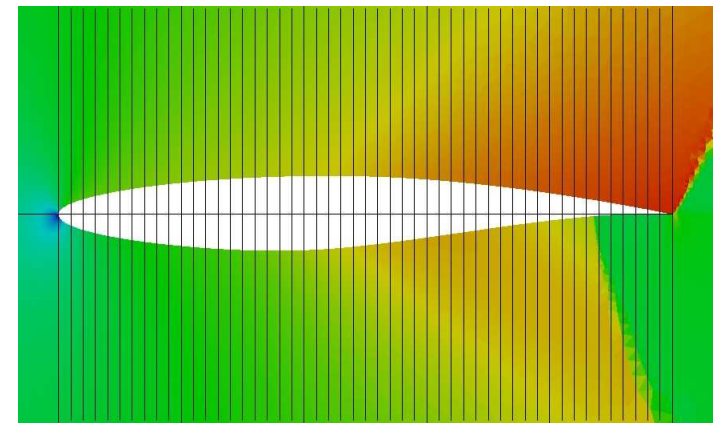

(c) Malha 3

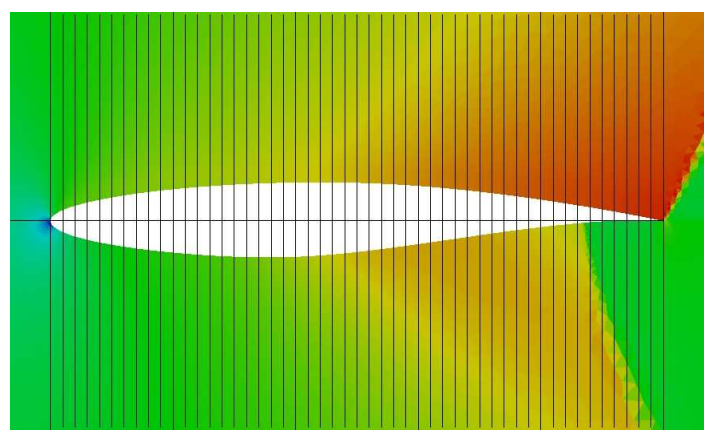

(b) Malha 2

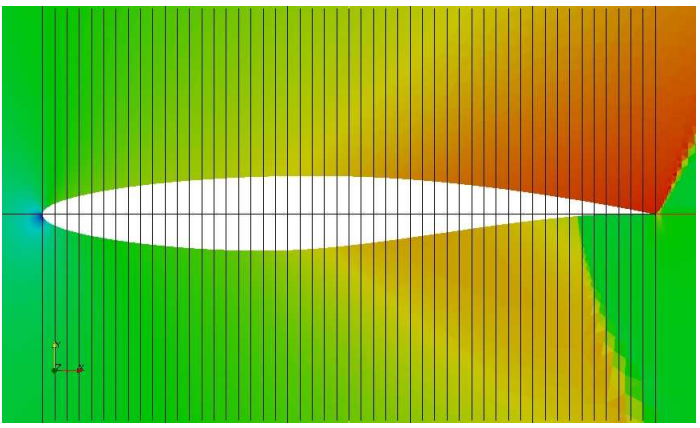

(d) Malha 4

Figura 4.44: Contornos de número de Mach do perfil RAE 2822 com número de Mach de escoamento livre, $M_{\infty}=0.9$, e ângulo de ataque, $\theta=3^{\circ}$.

É claro, que no presente trabalho não se ousa afirmar a não-unicidade da solução. Até mesmo porque, devido a limitação de tempo aqui encontrada, não foi possível realizar simulações em malhas mais finas, fato descrito na literatura como primordial para obter soluções estacionárias no regime de escoamento transônico. A questão, no entato, justifica de alguma forma, as discrepâncias reveladas em uma parte dos resultados.

As figuras 4.45 à 4.49 apresentam as sensibilidades do momento de arfagem em relação ao bordo de ataque do perfil RAE 2822 com relação aos mesmos parâmetros de controle.

Observa-se que as sensibilidades do momento de arfagem medidas pelo método adjunto são condizentes com diferenças finitas nas mesmas condições de escoamento livre que as sensibilidades da força de sustentação calculadas pelo método adjunto são coerentes com diferenças finitas. Essa constatação pode ser uma consequencia direta da dificuldade de posicionar choques em alguns casos devido ao possível excesso de dissipação artificial. 


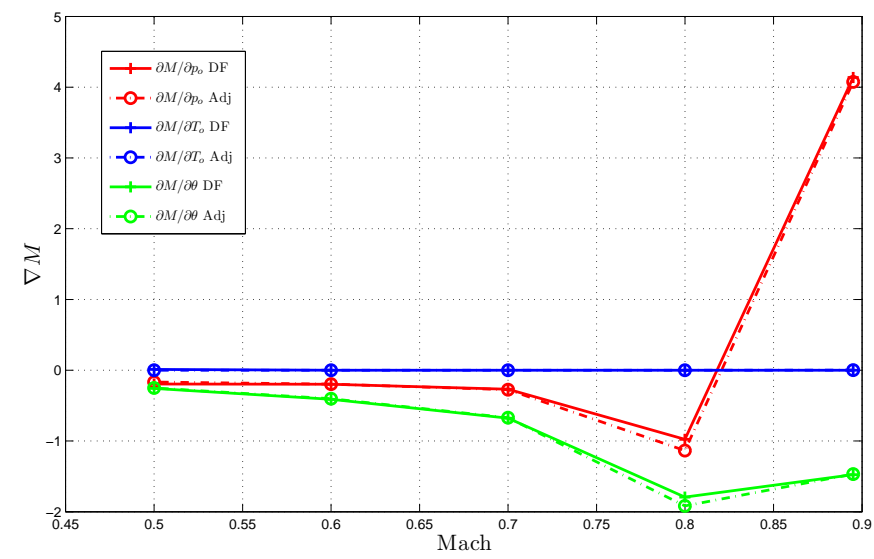

Figura 4.45: Sensibilidade do momento de arfagem em perfil RAE $2822 \mathrm{com}$ $\theta=0^{\circ}$. Linhas sólidas com marcador ' + ', diferenças finitas; traço-ponto com marcador 'o', adjunto. Vermelho, $\partial M / \partial p_{o}$; azul, $\partial M / \partial T_{o}$; verde, $\partial M / \partial \theta$.

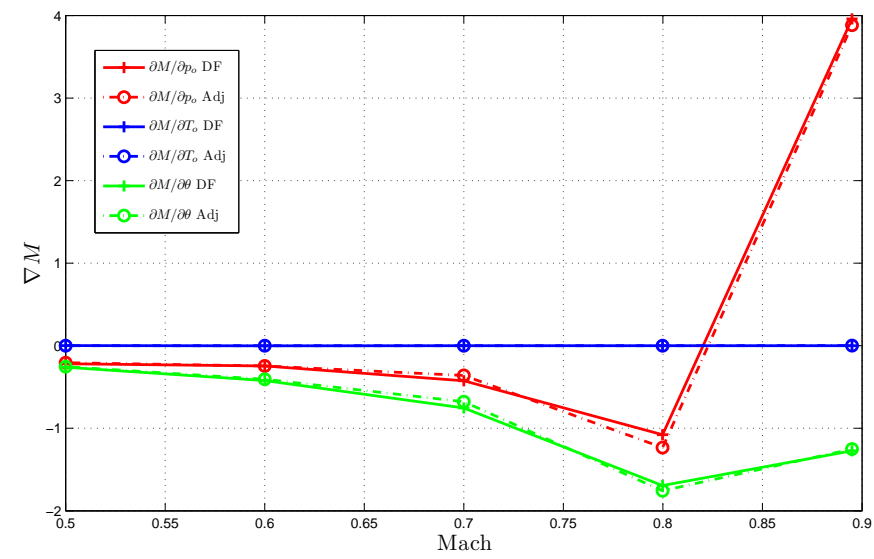

Figura 4.46: Sensibilidade do momento de arfagem em perfil RAE $2822 \mathrm{com}$ $\theta=1^{\circ}$. Linhas sólidas com marcador ' + ', diferenças finitas; traço-ponto com marcador 'o', adjunto. Vermelho, $\partial M / \partial p_{o}$; azul, $\partial M / \partial T_{o}$; verde, $\partial M / \partial M \theta$.

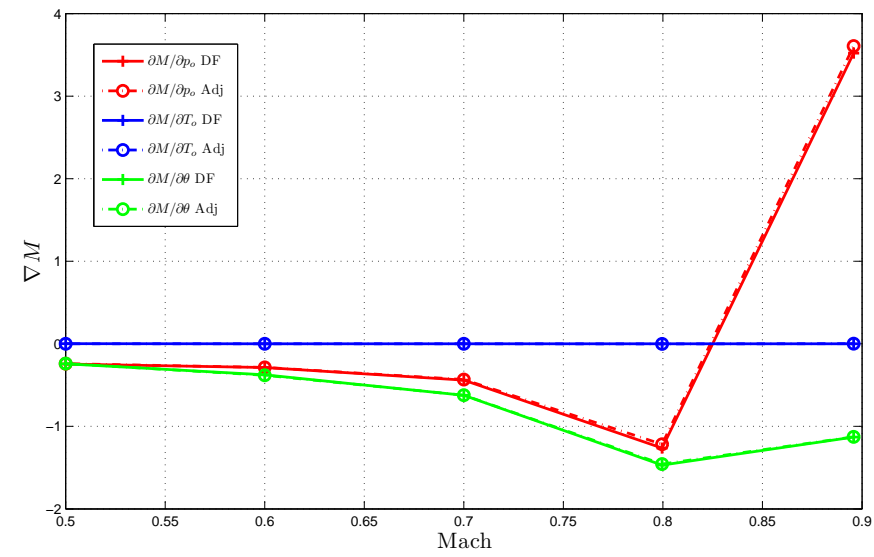

Figura 4.47: Sensibilidade do momento de arfagem em perfil RAE $2822 \mathrm{com}$ $\theta=2^{\circ}$. Linhas sólidas com marcador ' + ', diferenças finitas; traço-ponto com marcador 'o', adjunto. Vermelho, $\partial M / \partial p_{o}$; azul, $\partial M / \partial T_{o}$; verde, $\partial M / \partial \theta$. 


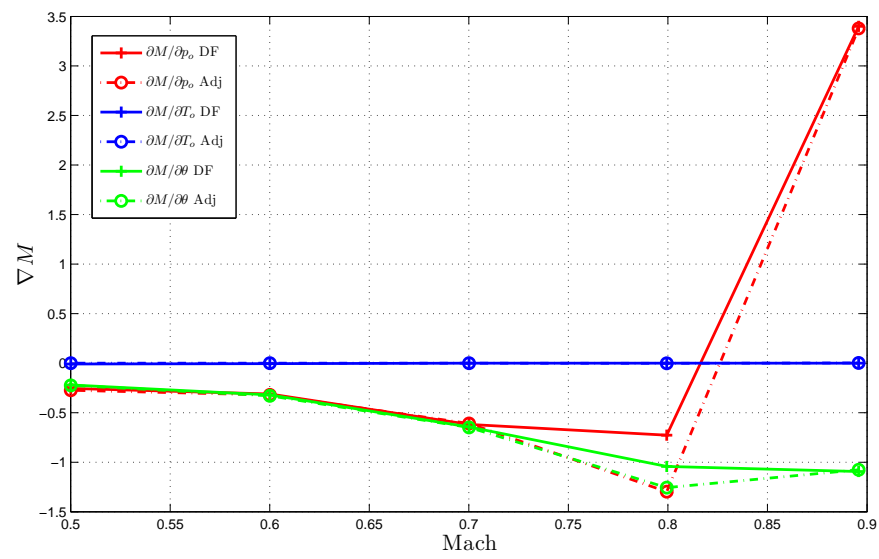

Figura 4.48: Sensibilidade do momento de arfagem em perfil RAE $2822 \mathrm{com}$ $\theta=3^{\circ}$. Linhas sólidas com marcador ' + ', diferenças finitas; traço-ponto com marcador 'o', adjunto. Vermelho, $\partial M / \partial p_{o}$; azul, $\partial M / \partial T_{o}$; verde, $\partial M / \partial \theta$.

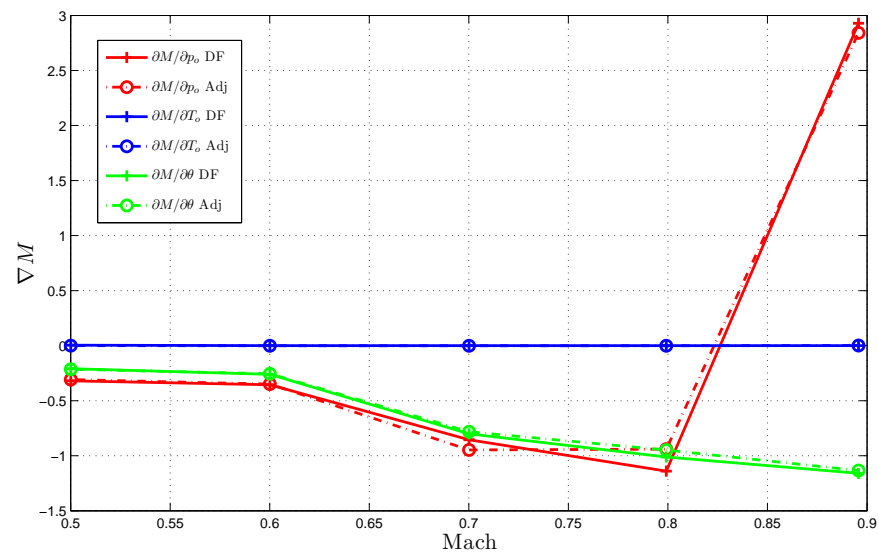

Figura 4.49: Sensibilidade do momento de arfagem em perfil RAE $2822 \mathrm{com}$ $\theta=4^{\circ}$. Linhas sólidas com marcador ' + ', diferenças finitas; traço-ponto com marcador 'o', adjunto. Vermelho, $\partial M / \partial p_{o}$; azul, $\partial M / \partial T_{o}$; verde, $\partial M / \partial \theta$.

\subsubsection{Perfil NACA 0012}

Por fim, a última geometria a ser considerada neste trabalho é o perfil simétrico NACA 0012. A figura 4.50 mostra a malha utilizada para as simulações com esta geometria, juntamente com os contornos de número de Mach para um caso em que $M_{\infty}=0.8$ e o ângulo de ataque é $\theta=2^{\circ}$. A solução adjunta para medida de mérito, $L$, desse mesmo caso é ilustrada na fig. 4.51 .

Por, fim, as figuras $4.52-4.56$ trazem os gradientes de sensibilidade para essa geometria em 5 diferentes ângulos de ataque. Note que ainda existem alguns problemas na comparação dos gradientes adjunto e força bruta no regime de escoamento transônico. Assim como na seção anterior, acredita-se que eles sejam oriundos de oscilações na posição do choque em uma solução que, a princípio, 


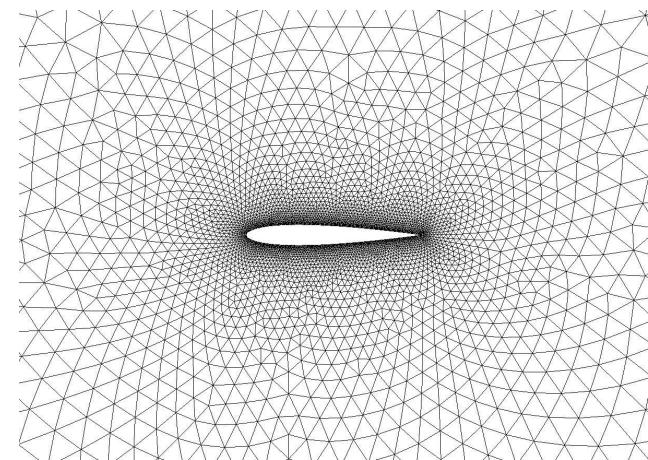

(a) Malha

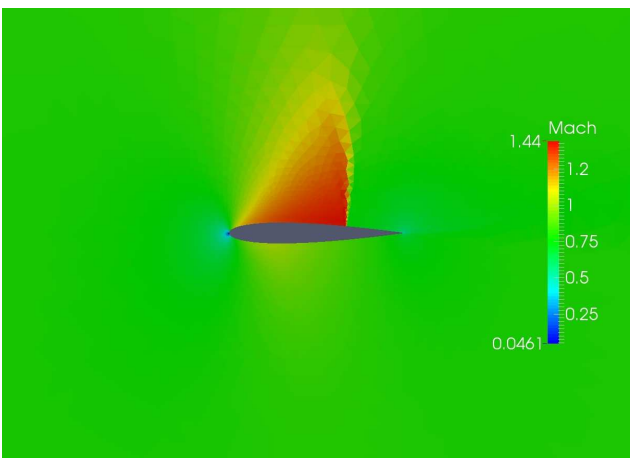

(b) Contornos de número de Mach

Figura 4.50: Solução do perfil NACA 0012 com número de Mach de escoamento livre, $M_{\infty}=0.8$, e ângulo de ataque, $\alpha=2^{\circ}$.

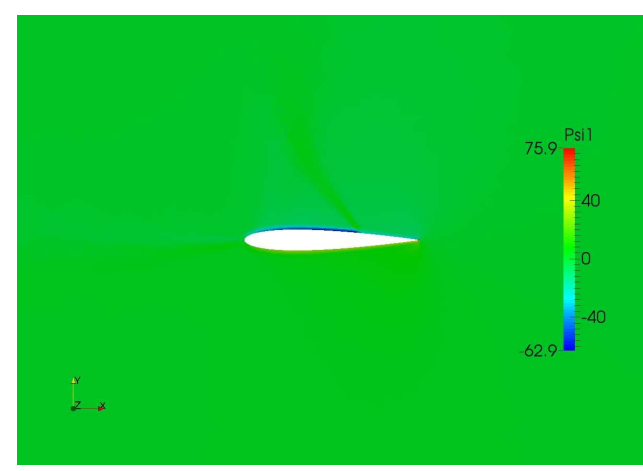

(a) $\varphi_{1}$

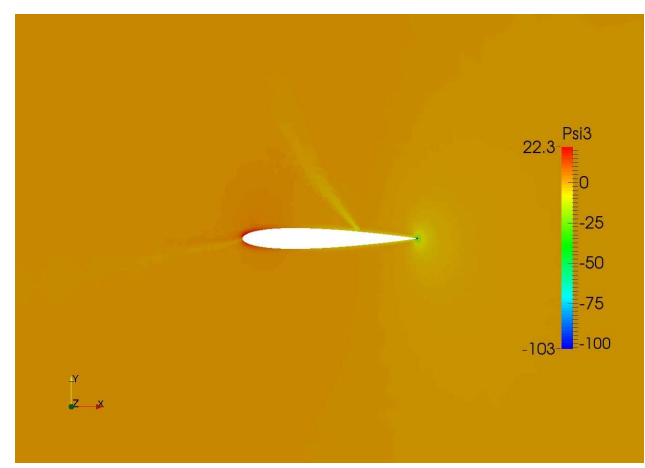

(c) $\varphi_{3}$

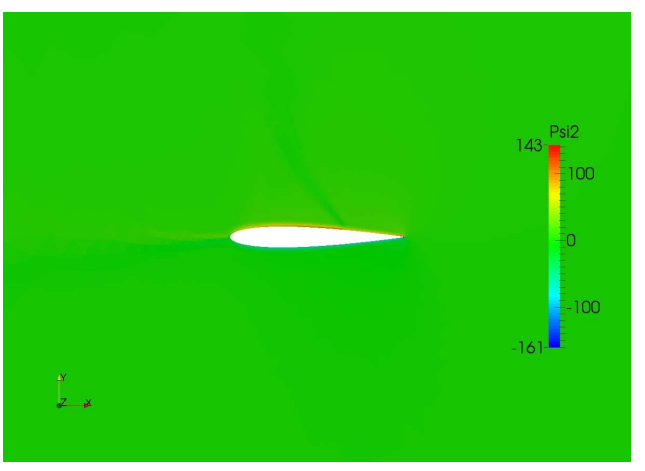

(b) $\varphi_{2}$

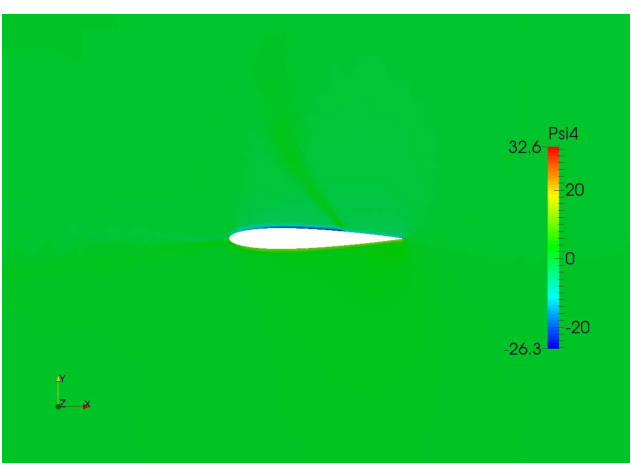

(d) $\varphi_{4}$

Figura 4.51: Solução adjunta do perfil NACA 0012 com $M=0.8$ e $\alpha=2^{\circ}$.

deveria ser estacionária.

Note ainda que, assim como ocorreu com o RAE 2822, os piores resultados no NACA 0012 sucedem quando Mach está em torno de 0.8 nas fronteiras de entrada no domínio. De fato, esta é uma condição de escoamento crítica no que diz respeito a unicidade de soluções (JAMESON, 1991).

Também é importante dizer que a presença de uma onda de choque no problema físico não deve ser um problema para o método. Basta observar os bons resultados em escoamentos supersônicos incidindo no perfil diamante na seção 


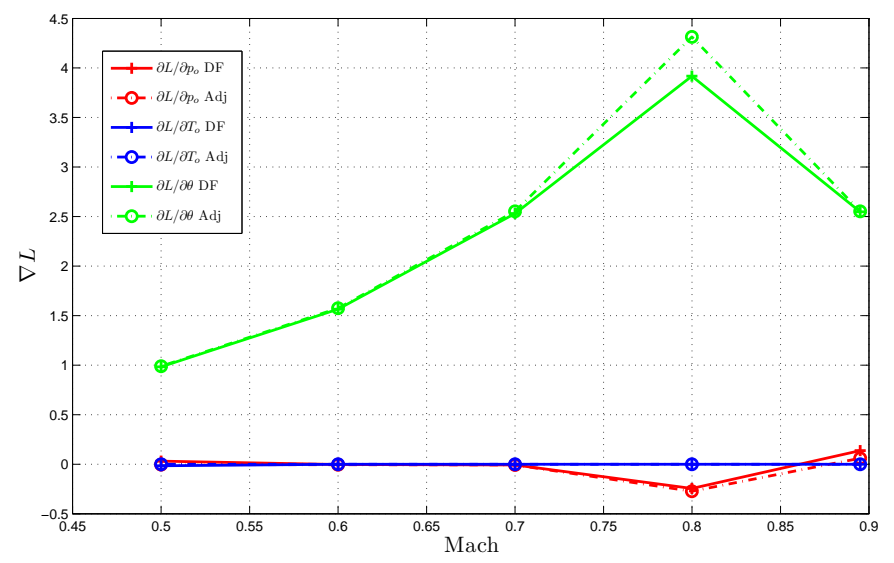

Figura 4.52: Sensibilidade da sustentação em perfil NACA $0012 \operatorname{com} \theta=0^{\circ}$. Linhas sólidas com marcador ' + ', diferenças finitas; traço-ponto com marcador 'o', adjunto. Vermelho, $\partial L / \partial p_{o}$; azul, $\partial L / \partial T_{o}$; verde, $\partial L / \partial \theta$.

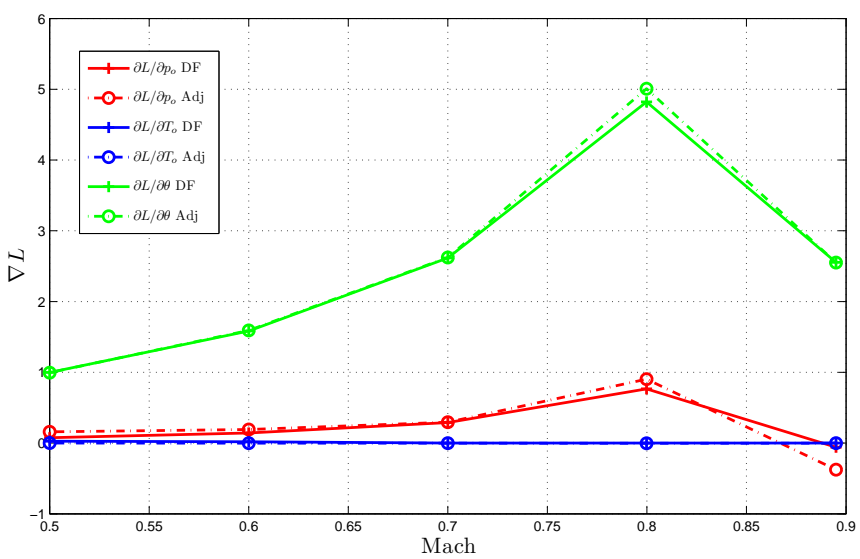

Figura 4.53: Sensibilidade da sustentação em perfil NACA $0012 \mathrm{com} \theta=1^{\circ}$. Linhas sólidas com marcador ' + ', diferenças finitas; traço-ponto com marcador 'o', adjunto. Vermelho, $\partial L / \partial p_{o}$; azul, $\partial L / \partial T_{o}$; verde, $\partial L / \partial \theta$.

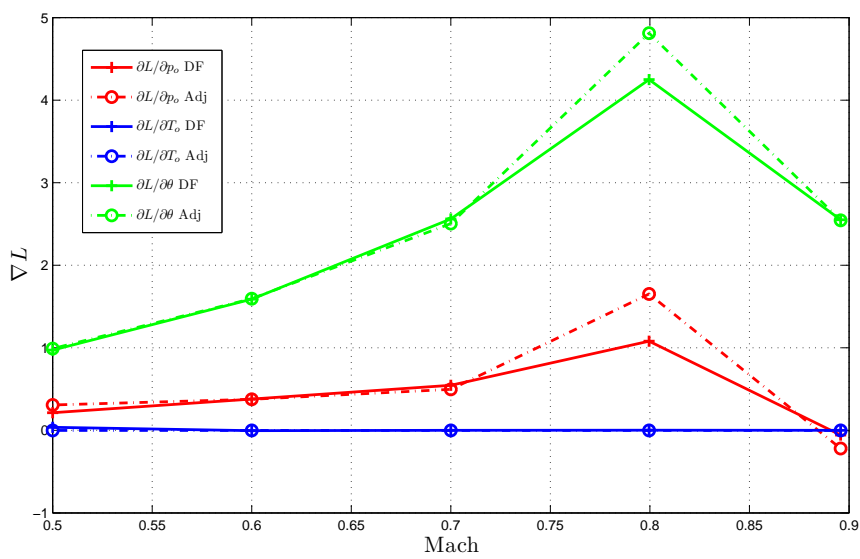

Figura 4.54: Sensibilidade da sustentação em perfil NACA $0012 \operatorname{com} \theta=2^{\circ}$. Linhas sólidas com marcador ' + ', diferenças finitas; traço-ponto com marcador 'o', adjunto. Vermelho, $\partial L / \partial p_{o}$; azul, $\partial L / \partial T_{o}$; verde, $\partial L / \partial \theta$. 


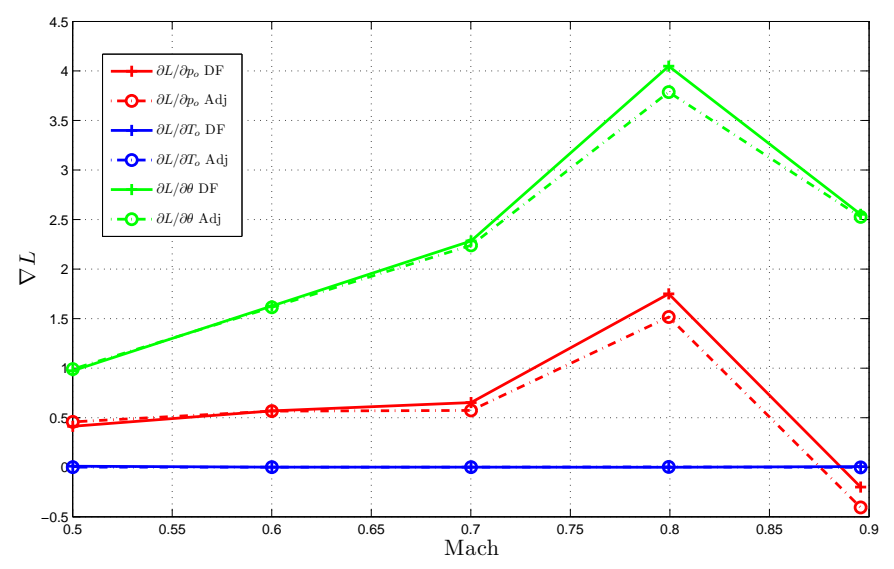

Figura 4.55: Sensibilidade da sustentação em perfil NACA $0012 \operatorname{com} \theta=3^{\circ}$. Linhas sólidas com marcador ' + ', diferenças finitas; traço-ponto com marcador 'o', adjunto. Vermelho, $\partial L / \partial p_{o}$; azul, $\partial L / \partial T_{o}$; verde, $\partial L / \partial \theta$.

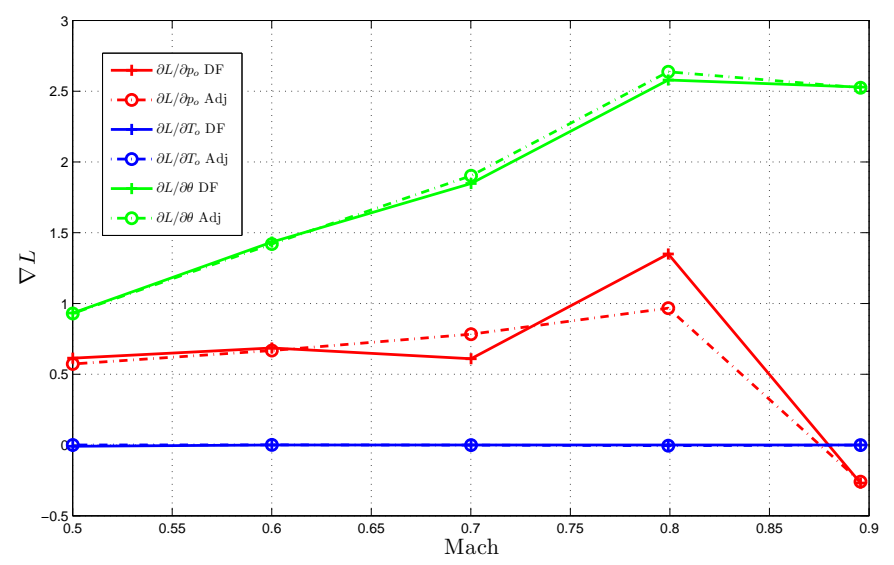

Figura 4.56: Sensibilidade da sustentação em perfil NACA $0012 \operatorname{com} \theta=4^{\circ}$. Linhas sólidas com marcador ' + ', diferenças finitas; traço-ponto com marcador 'o', adjunto. Vermelho, $\partial L / \partial p_{o}$; azul, $\partial L / \partial T_{o}$; verde, $\partial L / \partial \theta$.

4.4.1, em que haviam ondas de choque em todas as soluções. O que, aparentemente piora o cálculo dos gradientes são soluções múltiplas associadas a uma mesma condição.

Seria interessante analisar a solução problemática em diferentes malhas, assim como se fez com o perfil RAE 2822. Para isso, a tabela 4.4 enumera uma sequencia de 4 malhas progressivamente mais refinadas que foram geradas para estudar as soluções que envolvem esta geometria.

A região da malha próxima ao aerofólio é destacada na figura 4.57 para cada uma das discretizações espaciais descritas na tabela 4.4.

Seguindo o procedimento realizado anteriormente para o RAE 2822, propõese analisar agora o gradiente de sensibilidade $\partial L / \partial \theta$ nas diferentes malhas geradas para o NACA 0012 em simulações com ângulo de ataque $\theta=2^{\circ}$. A fig. 4.58 
Tabela 4.4: Malhas NACA0012

\begin{tabular}{ccc}
\hline \hline Malha & No $^{\circ}$ de elementos & $\mathrm{N}^{\mathrm{o}}$ de elementos no aerofólio \\
\hline 1 & 11238 & 300 \\
2 & 19308 & 400 \\
3 & 30474 & 800 \\
4 & 41220 & 1200 \\
\hline \hline
\end{tabular}

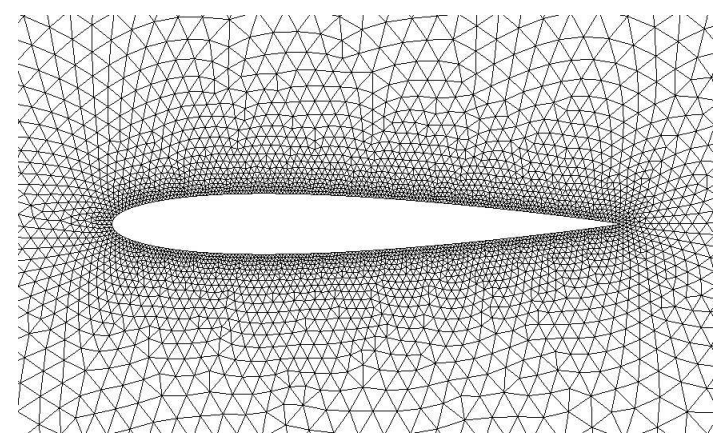

(a) Malha 1

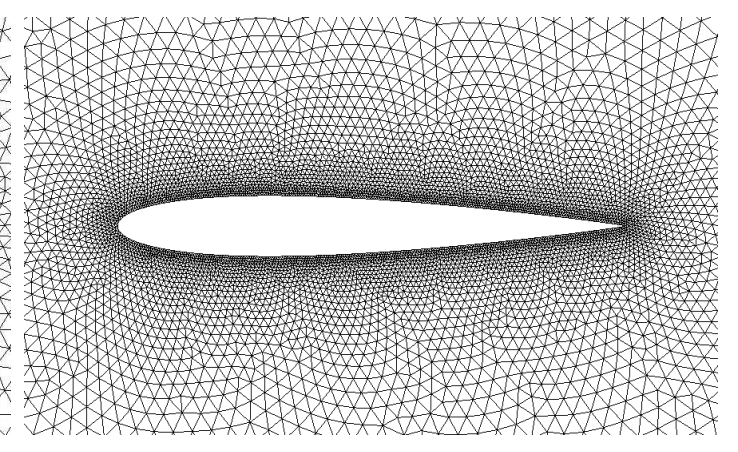

(b) Malha 2

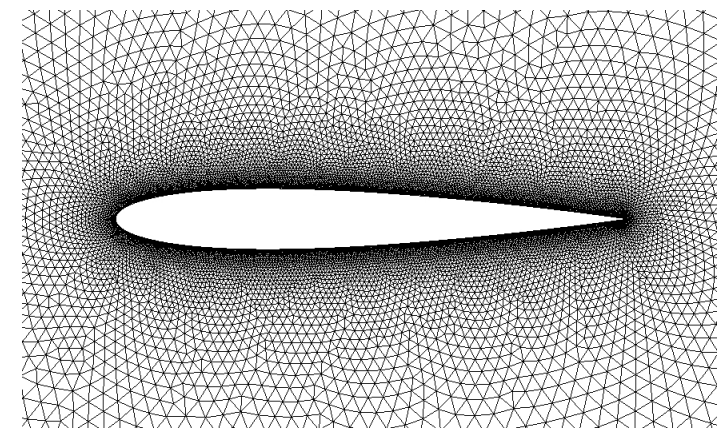

(c) Malha 3

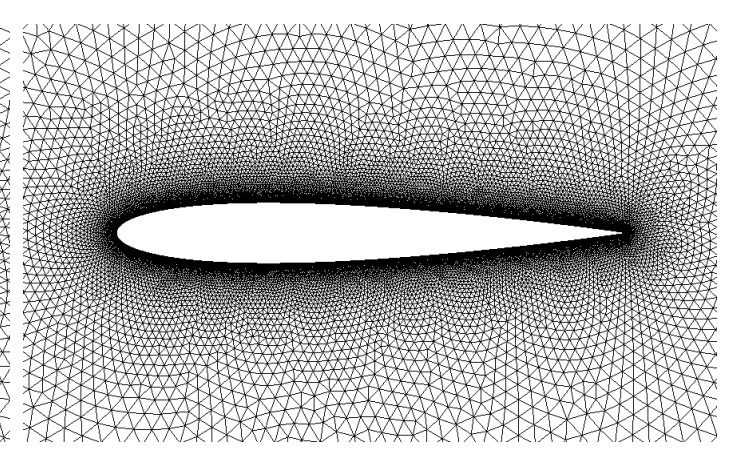

(d) Malha 4

Figura 4.57: Malhas NACA0012

apresenta, justamente, uma compilação desses resultados.

Observe, agora as soluções físicas do caso que tem número de Mach de escoamento livre, $M_{\infty}=0.8$, e ângulo de ataque, $\theta=2^{\circ}$, expostas na fig. (4.59). Note que, aqui a oscilação da posição do choque nas soluções estacionárias é ainda mais evidente do que no caso anterior. Talvez por isso as diferenças tenham sido também maiores.

A figura 4.60 apresenta os históricos de convergência das equações do movimento para cada uma das malhas do caso com número de Mach de escoamento livre, $M_{\infty}=0.8$, e ângulo de ataque, $\theta=2^{\circ}$. Assim como na seção anterior, todas as soluções parecem ter chegado ao estado estacionário.

E, novamente, a justificativa para os choques em posições diferentes pode ser explicada pela dissipação artificial, evidenciada pela geração de entropia ao redor 


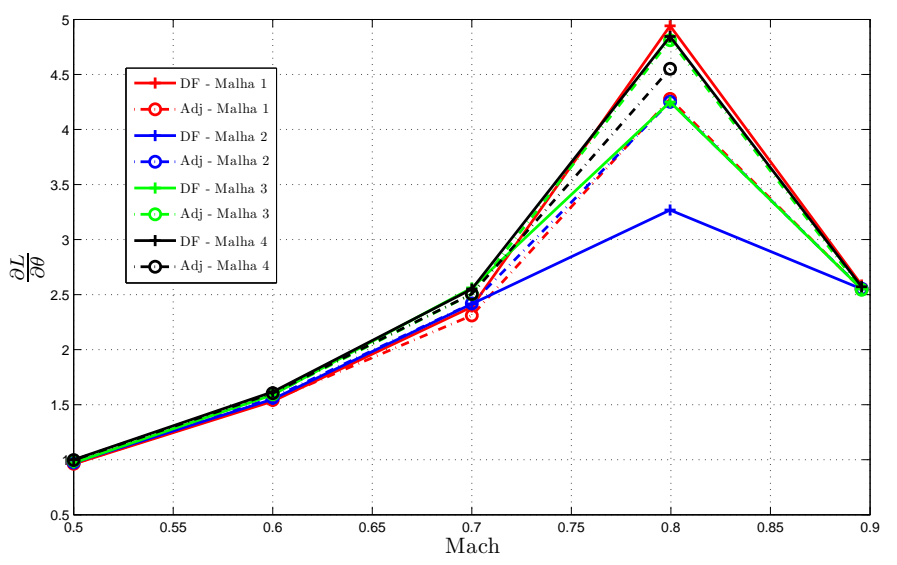

Figura 4.58: Comparação da sensibilidade $\partial L / \partial \theta$ em diferentes malhas do perfil NACA $0012 \operatorname{com} \theta=2^{\circ}$. Linhas sólidas com marcador ' + ', diferenças finitas; traço-ponto com marcador 'o', adjunto. Vermelho, malha 1; azul, malha 2; verde, malha 3 ; preto, malha 4.

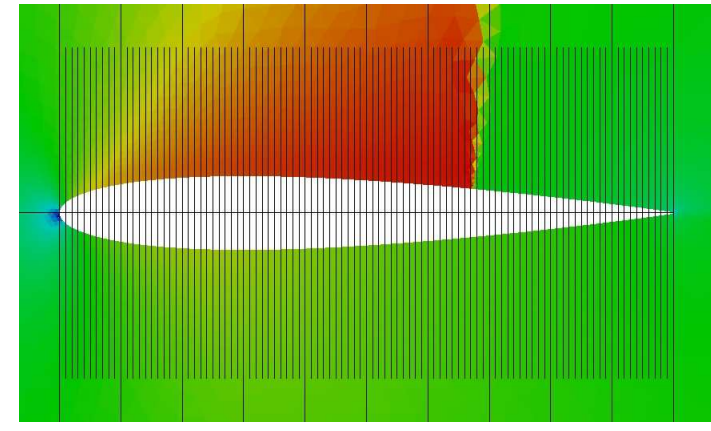

(a) Malha 1

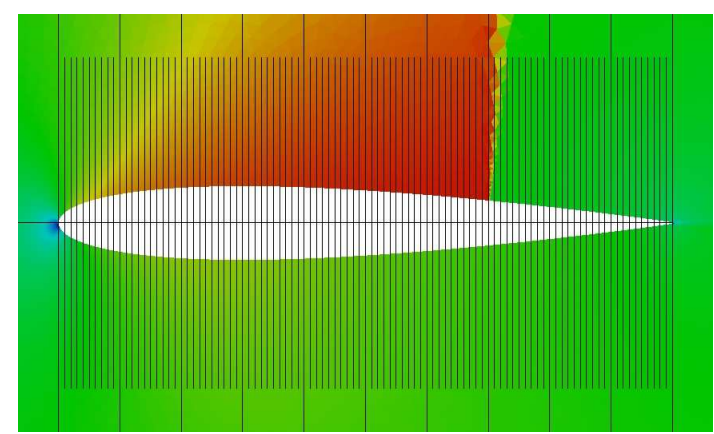

(c) Malha 3

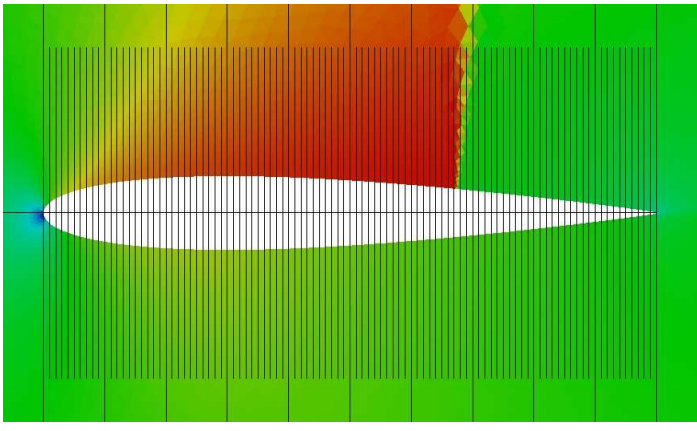

(b) Malha 2

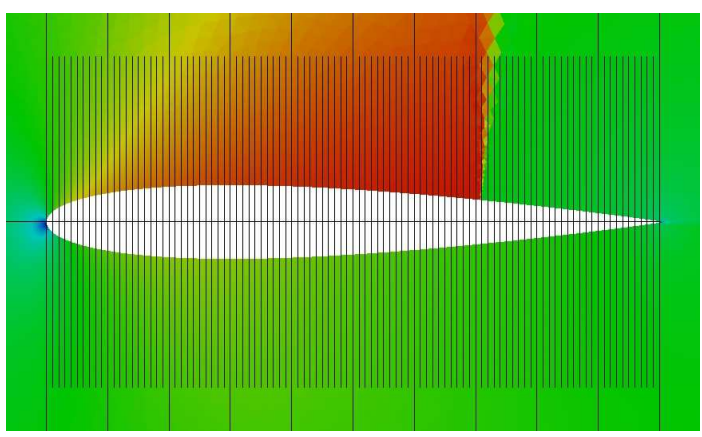

(d) Malha 4

Figura 4.59: Contornos de número de Mach do perfil NACA 0012 com número de Mach de escoamento livre, $M_{\infty}=0.8$, e ângulo de ataque, $\theta=2^{\circ}$.

dos aerofólios destacada na figura 4.61.

Similarmente ao que ocorreu com o RAE 2822, a condição de escoamento com $M_{\infty}=0.9$ também resultou em soluções estacionárias com choque fixo, indepenedentemente da malha como mostra a fig. 4.62, o que justifica os melhores resultados obtidos nesta condição.

Por fim, as figuras $4.63-4.67$ apresentam a comparação entre os gradientes 


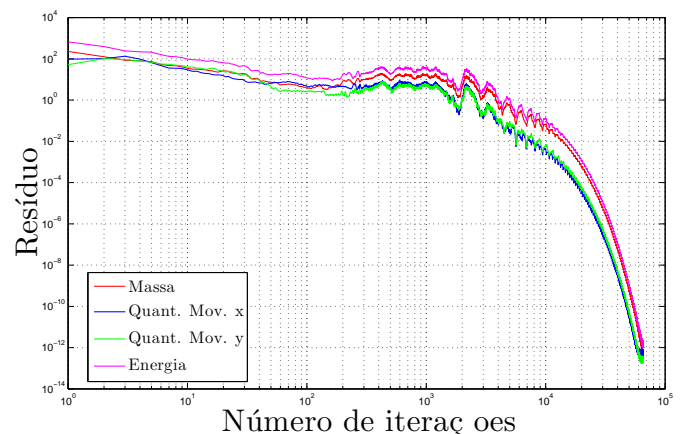

(a) Malha 1

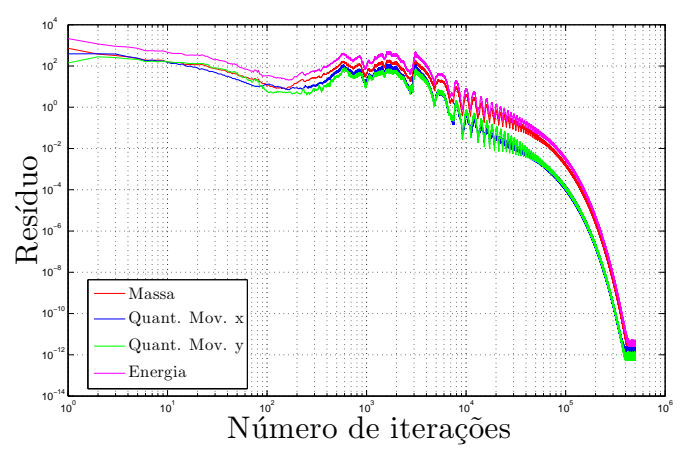

(c) Malha 3

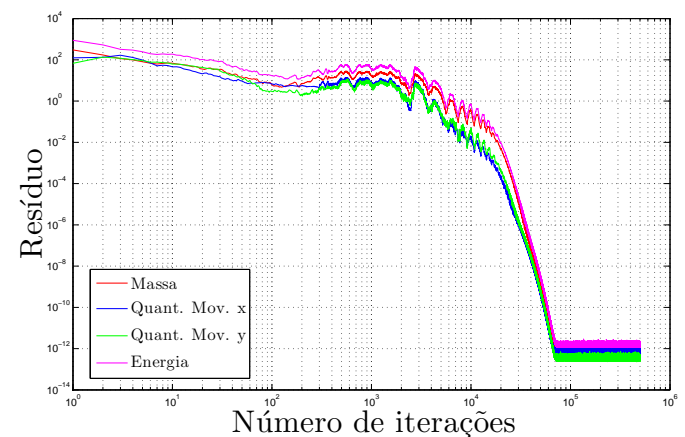

(b) Malha 2

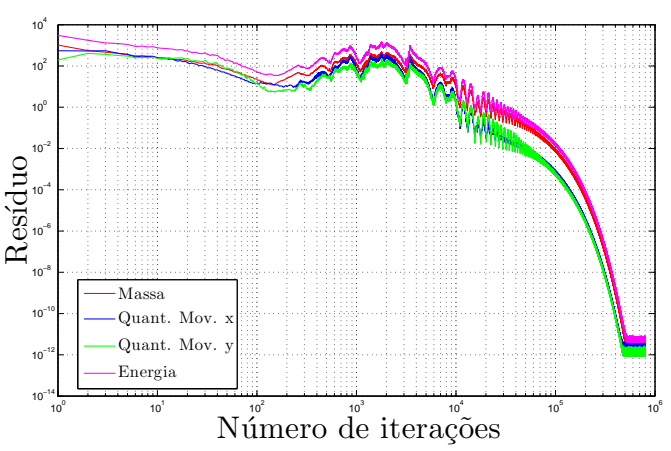

(d) Malha 4

Figura 4.60: Histórico de convergência das soluções físicas do perfil NACA 0012 com número de Mach de escoamento livre, $M_{\infty}=0.8$, e ângulo de ataque, $\theta=2^{\circ}$.

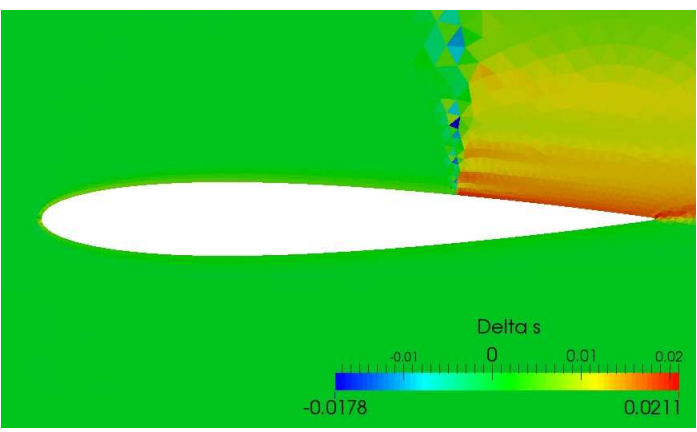

(a) Malha 1

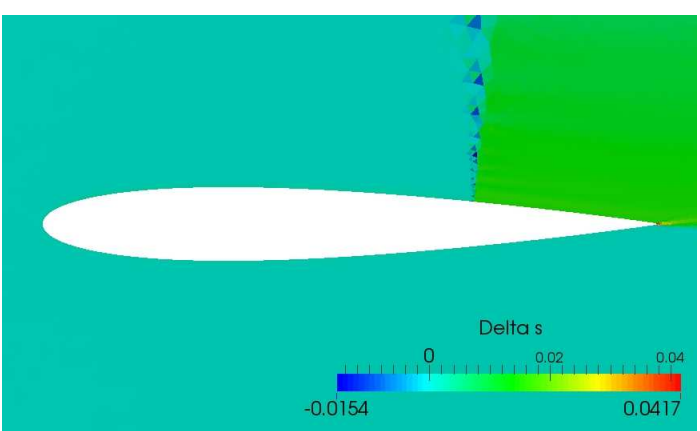

(c) Malha 3

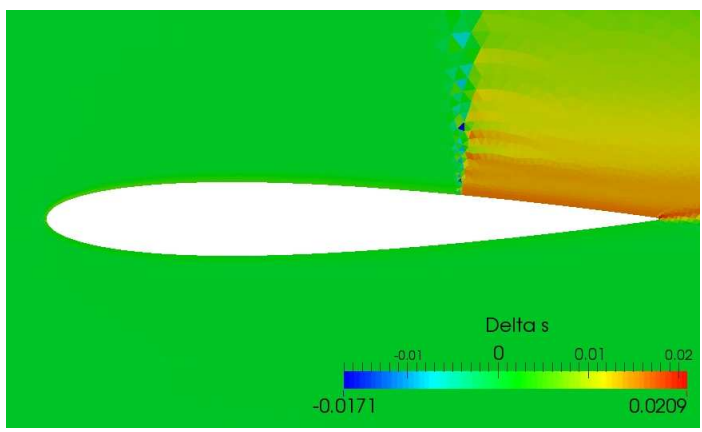

(b) Malha 2

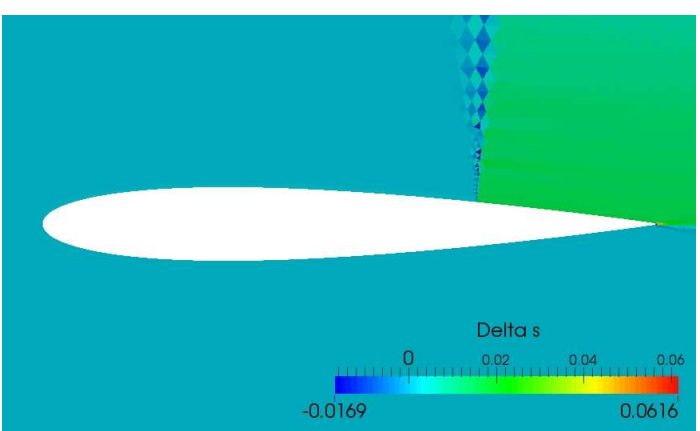

(d) Malha 4

Figura 4.61: Contornos de número de Mach do perfil NACA 0012 com número de Mach de escoamento livre, $M_{\infty}=0.8$, e ângulo de ataque, $\theta=2^{\circ}$. 


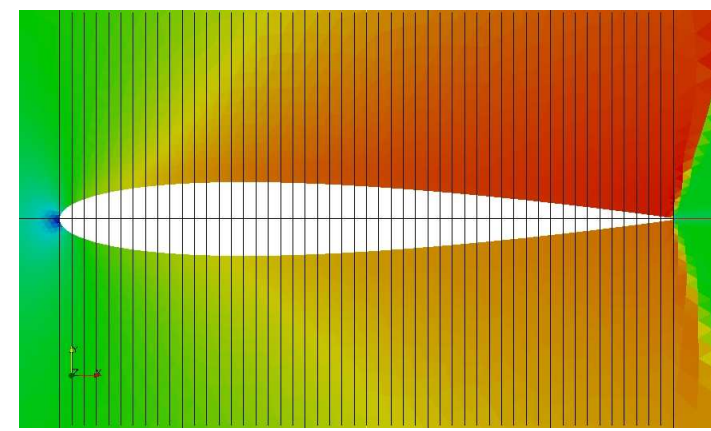

(a) Malha 1

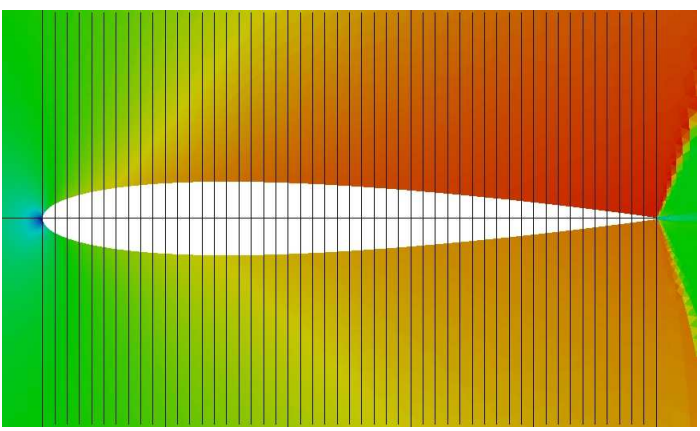

(c) Malha 3

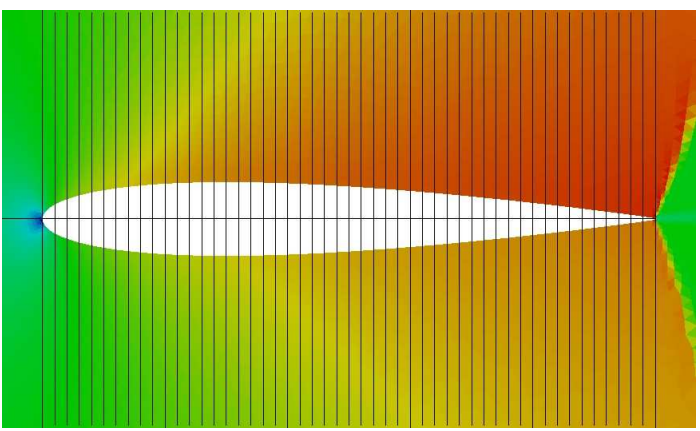

(b) Malha 2

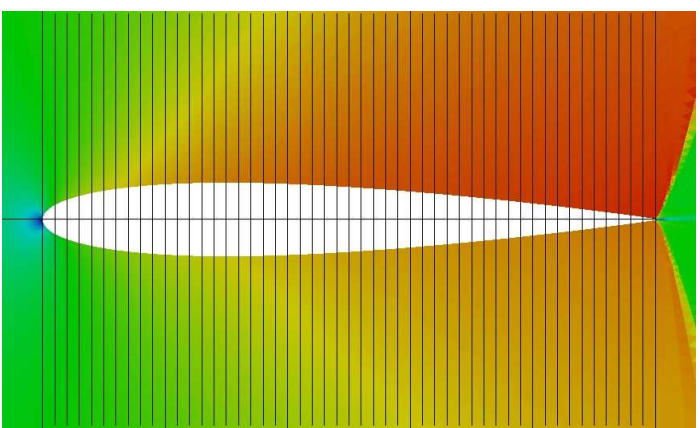

(d) Malha 4

Figura 4.62: Contornos de número de Mach do perfil NACA 0012 com número de Mach de escoamento livre, $M_{\infty}=0.9$, e ângulo de ataque, $\theta=2^{\circ}$.

obtidos pelo método adjunto e diferenças finitas do momento de arfagem. Novamente, os gradientes adjuntos são muito condizentes com os obtidos pelo método de força bruta nos casos inteiramente subsônicos, porém, não se pode dizer o mesmo dos casos transônicos. Particularmente, aqueles em que foram encontradas soluções diferentes para cada malha, com as mesmas condições de escoamento não perturbado.

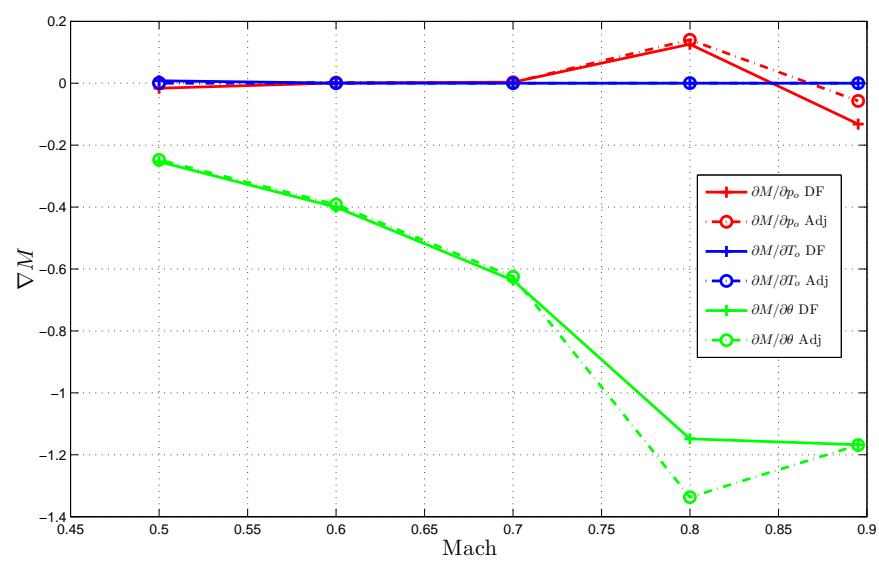

Figura 4.63: Sensibilidade do momento de arfagem em perfil NACA $0012 \mathrm{com}$ $\theta=0^{\circ}$. Linhas sólidas com marcador ' + ', diferenças finitas; traço-ponto com marcador 'o', adjunto. Vermelho, $\partial M / \partial p_{o}$; azul, $\partial M / \partial T_{o}$; verde, $\partial M / \partial \theta$. 


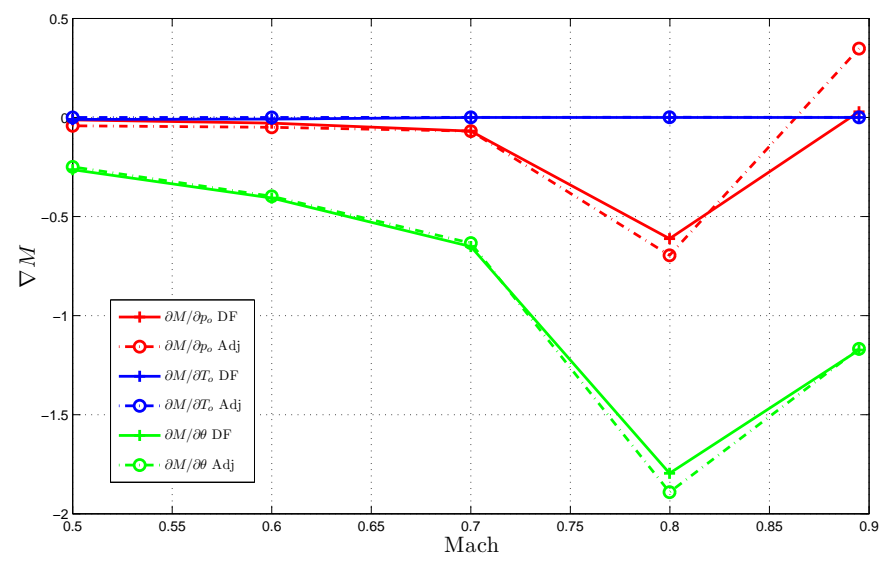

Figura 4.64: Sensibilidade do momento de arfagem em perfil NACA $0012 \mathrm{com}$ $\theta=1^{\circ}$. Linhas sólidas com marcador ' + ', diferenças finitas; traço-ponto com marcador 'o', adjunto. Vermelho, $\partial M / \partial p_{o}$; azul, $\partial M / \partial T_{o}$; verde, $\partial M / \partial \theta$.

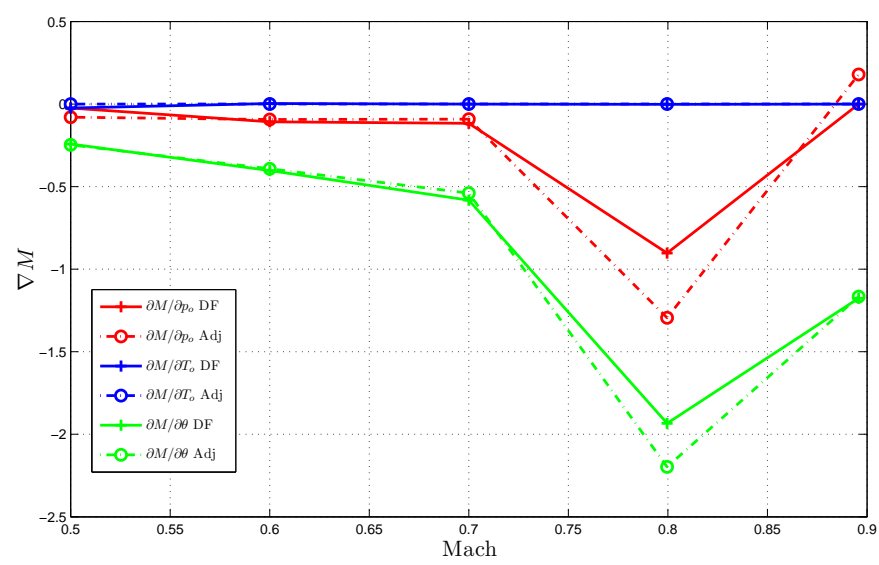

Figura 4.65: Sensibilidade do momento de arfagem em perfil NACA $0012 \mathrm{com}$ $\theta=2^{\circ}$. Linhas sólidas com marcador ' + ', diferenças finitas; traço-ponto com marcador 'o', adjunto. Vermelho, $\partial M / \partial p_{o}$; azul, $\partial M / \partial T_{o}$; verde, $\partial M / \partial \theta$.

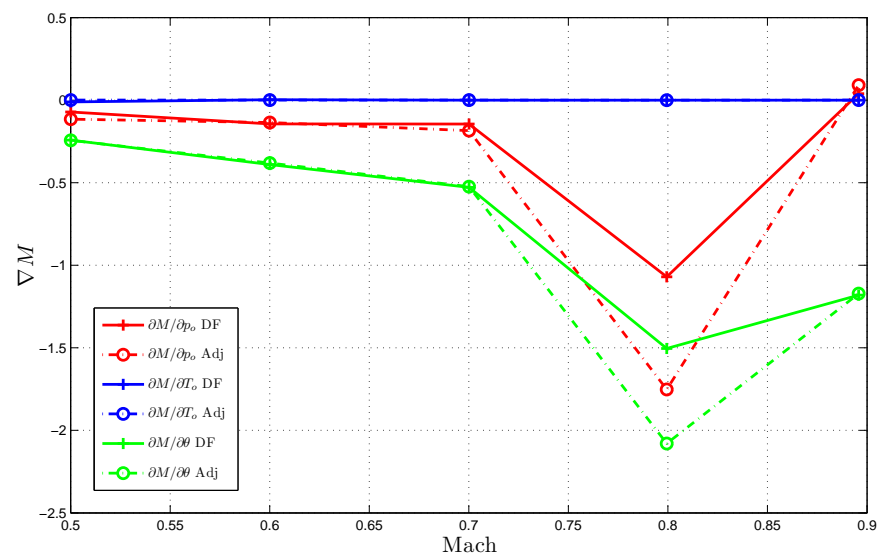

Figura 4.66: Sensibilidade do momento de arfagem em perfil NACA $0012 \mathrm{com}$ $\theta=3^{\circ}$. Linhas sólidas com marcador ' + ', diferenças finitas; traço-ponto com marcador 'o', adjunto. Vermelho, $\partial M / \partial p_{o}$; azul, $\partial M / \partial T_{o}$; verde, $\partial M / \partial \theta$. 


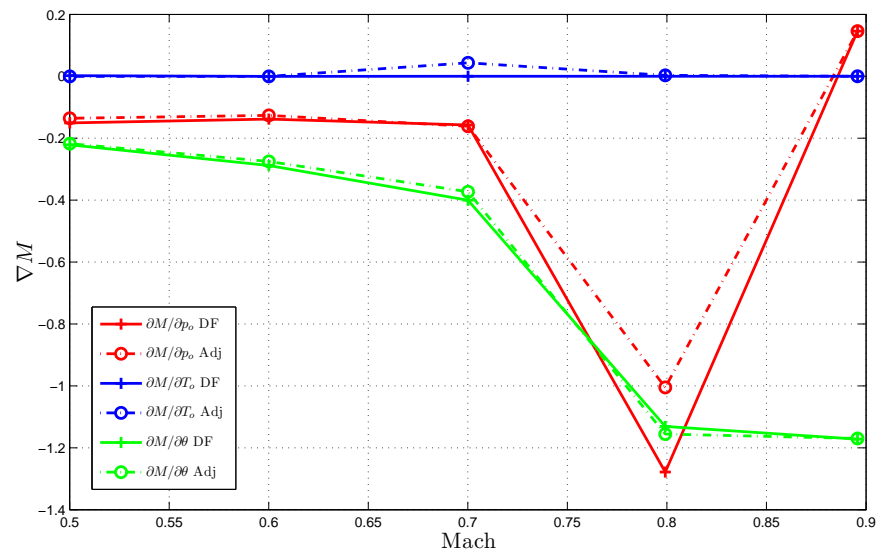

Figura 4.67: Sensibilidade do momento de arfagem em perfil NACA $0012 \mathrm{com}$ $\theta=4^{\circ}$. Linhas sólidas com marcador ' + ', diferenças finitas; traço-ponto com marcador 'o', adjunto. Vermelho, $\partial M / \partial p_{o}$; azul, $\partial M / \partial T_{o}$; verde, $\partial M / \partial \theta$. 


\section{Conclusões E TRABAlHos FUTUROS}

\subsection{Conclusões}

Este trabalho propõe uma nova abordagem da formulação contínua do método adjunto, que permite estender a sua, já conhecida, capacidade de estimar sensibilidades de medidas de mérito para aplicações aerodinâmicas. Ao utilizar propriedades físicas do escoamento como parâmetros de controle do problema de otimização em fronteiras permeáveis do domínio computacional, o procedimento, originalmente apresentado por Cacuci et al. (1980) para avaliar sensibilidades gerais de sistemas de equações não-lineares, permite calcular as chamadas derivadas de estabilidade de uma aeronave, por exemplo. O procedimento, no entanto, requer que o problema de contorno adjunto seja construído de modo consistente com a decomposição do concomitante bilinear proposta por Cacuci et al. (1980). Verificou-se, então, que as condições de contorno desenvolvidas por Hayashi, Ceze e Volpe (2013) são compatíveis com este procedimento. Consequentemente, gradientes não-geométricos, poderiam ser estimados com a mesma solução adjunta calculada para realizar otimização de forma. Vale ressaltar que a implementação das usuais condições de contorno homogêneas adjuntas utilizadas em problemas de aerodinâmica externa resultariam em gradientes nulos com relação a qualquer parâmetro em fronteiras de farfield, como se pode observar nas equações (3.72) e (3.71), que se referem a essa informação nos regimes de escoamento subsônico e supersônico, respectivamente.

As aplicações apresentadas nesta tese se limitam a escoamentos modelados pelas equações de Euler bidimensionais em estado estacionário. Essa escolha se deve, naturalmente, às restrições de tempo e adequação ao escopo do trabalho associado a uma tese de doutoramento. É importante dizer, no entanto, que do ponto de vista teórico, o mesmo procedimento pode ser aplicado a escoamentos tridimensionais. Gradientes adjuntos de diferentes medidas de mérito foram validados por comparação com os obtidos pelo método diferenças finitas em casos de 
escoamento interno e externo nos regimes subsônico, supersônico e transônico. O método se mostrou muito eficaz nos casos de escoamento subsônico e supersônico sem a presença de choques. Os testes supersônicos com ondas de choque coladas ao perfil diamante também demonstraram uma forte concordância entre os gradientes obtidos por diferenças finitas e o método adjunto. Já nos casos transônicos, nem todos os resultados apresentaram o mesmo grau de concordância entre os gradientes. Para tentar contornar esse problema foi feito um estudo de refinamento de malha. Aparentemente, como foi discutido no capítulo 4, verificou-se a existência de múltiplas soluções para uma mesma geometria. Isto ocorrreu em alguns casos em que o número de Mach do escoamento não perturbado estava em torno de 0.8. Mostrou-se, ainda, que existem diferenças na geração de entropia nos campos de solução fora do choque, o que indica a presença de dissipação artificial. Assim, é esperado que as soluções de malhas diferentes resultem em ondas de choque em posições também diferentes e, uma vez que a solução base não corresponde ao mesmo problema físico, não deveria ser possível comparar os gradientes. Aqui, vale ressaltar que não existe prova matemática de que haja unicidade na solução das equações de Euler em regime transônico. Esta ainda é uma questão aberta. Jameson (1991) relata, inclusive, ter encontrado múltiplas soluções em aerofólios para escoamentos modelados pelas equações de Euler numa faixa de número de Mach entre 0.75 e 0.8, números muito próximos aos dos casos problemáticos do capítulo 4. É possível que este problema seja resolvido ao utilizar malhas ainda mais refinadas, mas que por limitações de tempo não foram incluídas aqui. De qualquer forma, isso justifica, em algum grau, a discordância entre os gradientes adjunto e de diferenças finitas nesses casos.

Vale destacar ainda que a utilização da formulação contínua do método traz como grande trunfo a possibilidade de acoplá-lo a qualquer código que resolva o escoamento de fluidos modelados pelas equações de Euler compressíveis. Note que as variações físicas realizáveis descritas para anular o concomitante bilinear no problema variacional não requerem um código que resolva as equações governantes com a mesma imposição. Assim, poderia-se determinar o gradiente, inclusive, a partir de soluções físicas que tenham sido obtidas com invariantes de Riemann como condição de contorno nas fronteiras de farfield.

\subsection{Trabalhos Futuros}

A evidência de que as imprecisões enfrentadas durante a validação do método adjunto por comparação com gradientes obtidos pelo método de diferenças finitas 
se deve ao excesso de dissipação artificial que pode, em princípio, levar a ondas de choque posicionadas em regiões distintas, encaminham as pesquisas para uma direção de estudo de refinamento de malha. Vale destacar que o malhas mais refinadas implicam em menos dissipação artificial na solução numérica que, por hipótese, deve estar provocando o deslocamento das ondas de choque no extradorso dos perfis de asa. Isso inclui entre outras coisas a idéia de utilizar malhas adaptativas. Outra possibilidade, seguindo a mesma linha de pensamento, seria implementar algum método menos dissipativo para resolver as equações que governam o escoamento.

Pode-se considerar, ainda, de substituir o método de diferenças finitas pelo método de passo complexo (complex step), para comparação com o gradiente adjunto. Segundo Martins e Hwang (2013), ele substitui, com vantagens, o método de diferenças finitas para avaliação de derivadas parciais. 


\section{REFERÊNCIAS}

ALONSO, J. J.; KROO, I. M. Advanced algorithms for design and optimization of quiet supersonic platforms. In: AIAA Computational Fluid Dynamics Conference. Reno, NV: [s.n.], 2002. AIAA-2002-0144.

ANDERSON, J. D. Fundamentals of Aerodynamics. 5th. ed. N.Y.: McGraw-Hill, 2011. (McGraw-Hill series in aeronautical and aerospace engineering).

AZEVEDO, J. L. F. Notas de aula. Métodos Numéricos para Escoamento Compressível I. 2006.

BEUX, F.; DERVIEUX, A. A Hierarchical Approacch for Shape Optimization. [S.l.], 1993.

CACUCI, D. G.; WEBER; F., C.; M., O. E.; MARABLE, J. H. Sensitivity theory for general systems of non-linear equations. Nuclear Science and Engineering, v. 75 , p. $88-110,1980$.

CEZE, M. A. B. Projeto Inverso Aerodinâmico Utilizando o Método Adjunto Aplicado às Equações de Euler. Dissertação (Mestrado) - Escola Politécnica da USP, 2008.

CHIEREGATTI, B. G. Otimização aerodinâmica de aerofólios utilizando o método adjunto. Trabalho de Graduação - EPUSP. 2008.

DUTA, M. C.; GILES, M. B.; CAMPOBASSO, M. S. The harmonic adjoint approach to unsteady turbomachinery design. Int. J. Numer. Meth. Fluids, v. 40, p. 323-332, 2002.

ECONOMON, T. D.; PALACIOS, F.; ALONSO, J. J. An unsteady continuous adjoint approach for aerodynamic design on dynamic meshes. In: AIAA. 15th AIAA/ISSMO Multidisciplinary Analysis and Optimization Conference. Atlanta, GA, 2014.

FLÜGGE, W. Tensor Analysis and Continuum Mechanics. 1st. ed. N.Y.: Springer-Verlag, 1972.

FUJITA, T. Efficient wing design for complex aircraft configuration using cad, unstructured cfd and inverse problems. In: ICAS. ICAS 2002 Congress. [S.l.], 2002. p. 231.1-231.9.

GILES, M.; PIERCE, N.; SÜLI, E. Progress in adjoint error correction for integral functionals. Comput Visual Sci, v. 6, p. 113-121, 2004.

GILES, M.; SÜLI, E. Adjoint methods for pdes: a posteriori error analysis and postprocessing by duality. Acta Numerica, v. 11, p. 145-236, 2002. 
GILES, M. B.; PIERCE, N. A. Adjoint equations in cfd: duality, boundary conditions and solution behaviour. In: AIAA. 13th AIAA Computational Fluid Dynamics Conference. Snowmass Village, CO, USA, 1997.

GILES, M. B.; PIERCE, N. A. On the properties of the adjoint euler equations. In: BAINES, M. J. (Ed.). Numerical Methods for Fluid Dynamics VI. [S.l.: s.n.], 1998.

GILES, M. B.; PIERCE, N. A. Superconvergent lift estimates through adjoint error analysis. 1998.

GILES, M. B.; PIERCE, N. A. Adjoint recovery of superconvergent functionals from approximate solutions of partial differential equations. Oxford, August 1999.

GILES, M. B.; PIERCE, N. A. Improved lift and drag estimates using adjoint Euler equations. 1999. AIAA.

GILES, M. B.; PIERCE, N. A. Analytic Adjoint Solutions for the Quasi-1D Euler Equations. Oxford, March 2000.

GILES, M. B.; PIERCE, N. A. An introduction to the adjoint approach to design. Flow, Turbulence and Combustion, v. 65, p. 393-415, 2000.

GILES, M. B.; PIERCE, N. A. Analytic adjoint solutions for the quasi-1d Euler equations. Journal of Fluid Mechanics, v. 426, p. 327-345, 2001.

GOLDBERG, D. E. Genetic Algorithms in Search, Optimization and Machine Learning. 1st. ed. Boston, MA, USA: Addison-Wesley Longman Publishing Co., Inc., 1989.

HALL, M.; CACUCI, D. Physical interpretation of the adjoint functions for sensitivity analysis of atmospheric models. Journal of Atmospheric Sciences, v. 40, p. 2537-2546, October 1983.

HAYASHI, M.; CEZE, M.; VOLPE, E. Characteristics-based boundary conditions for the Euler adjoint problem. International Journal for Numerical Methods in Fluids, v. 71, n. 10, p. 1297-1321, April 2013.

HAYASHI, M. T. Estudo Conceitual do Problema Adjunto Baseado nas Equações de Euler Para Aplicações de Otimização Aerodinâmica. Dissertação (Mestrado)

- Escola Politécnica da USP, 2009.

HICKS, R. M.; HENNE, P. A. Wing design by numerical optimization. Journal of Aircraft, v. 15, n. 7, p. 407-412, July 1978.

HICKS, R. M.; MURMAN, E. M.; VANDERPLAATS, G. N. An Assessment of Airfoil Design by Numerical Optimization. Moffett Field, CA, July 1974. NASA TM X-3092.

HIRSCH, C. Numerical Computation of Internal and External Flows. Vol. 1: Fundamentals of Numerical Discretization. [S.l.]: John Wiley \& Sons, 1988.

HIRSCH, C. Numerical Computation of Internal and External Flows. Vol. 2: Computational Methods for Inviscid and Viscous Flows. [S.1.]: John Wiley \& Sons, 1988. 
HIRSCH, C. Numerical Computation of Internal and External Flows. Vol. 1: The Fundamentals of Computational Fluid Dynamics. 2nd. ed. [S.1.]: John Wiley \& Sons Ltd., 2007.

JAMESON, A. Aerodynamic design via control theory. In: 12th IMACS World Congress on Scientific Computation. Paris: [s.n.], 1988. (MAE Report 1824).

JAMESON, A. Automatic design of transonic airfoils to reduce the shock induced pressure drag. In: 31st Israel Annual Conference on Aviation and Aeronautics. Tel Aviv: [s.n.], 1990. (MAE Report 1881).

JAMESON, A. Airfoils admitting non-unique solutions of the euler equations. In: AIAA. AIAA 22nd Fluid Dynamics, Plasmadynamics and Laser Conference. Honolulu, HI, 1991.

JAMESON, A. Optimum Aerodynamic Design via Boundary Control. [S.l.], April 1994.

JAMESON, A. Re-engineering the design process through computation. In: 35th Aerospace Sciences Meeting \& Exhibit. Reno, NV: AIAA, 1997. AIAA-97-0641.

JAMESON, A. Aerodynamic Shape Optimization Using the Adjoint Method. February 2003. Lectures at the Von Kármán Institute, Brussels.

JAMESON, A.; KIM, S. Reduction of the adjoint gradient formula for aerodynamic shape optimization problems. AIAA Journal, v. 41, n. 11, p. 2114-2129, November 2003.

JAMESON, A.; KIM, S. Reduction of the adjoint gradient formula in the continuous limit. In: AIAA Computational Fluid Dynamics Conference. [S.l.: s.n.], 2003. AIAA 2003-0040.

JAMESON, A.; MARTINELLI, L.; PIERCE, N. A. Optimum aerodynamic design using the Navier-Stokes equations. In: AMERICAN INSTITUTE OF AERONAUTICS AND ASTRONAUTICS. 35th Aerospace Sciences Meeting 86 Exhibit. Reno, NV: AIAA, 1997. p. 1-20.

JAMESON, A.; NADARAJAH, S. K. A comparison of the continuous and discrete adjoint approach to automatic aerodynamic optimization. In: AIAA 38th Aerospace Sciences Meeting and Exhibit. Reno, NV: [s.n.], 2000.

JAMESON, A.; SCHMIDT, W.; TURKEL, E. Numerical solution of the euler equations by finite volume methods using runge-kutta time-stepping schemes. AIAA Journal, 1981.

JAMESON, A.; SRIRAM; MARTINELLI, L. A continuous adjoint method for unstructured grids. In: AIAA. 16th CFD Conference. Orlando, FL, 2003. AIAA 2003-3955.

KAPLAN, W. Advanced Calculus. NY: Addison-Wesley Pub. Co., 1971.

KIM, H. J.; KOC, S.; NAKAHASHI, K. Aerodynamic design of wing-bodynacelle-pylon configuration. In: AIAA Computational Fluid Dynamics Conference. [S.l.: s.n.], 2005. 
KIM, S. Design Optimization of High-Lift Configurations Using a Viscous Adjoint-Based Method. Tese (Doutorado) — Stanford University, 2001.

KLINE, H.; PALACIOS, F.; ECONOMON, T. D.; ALONSO, J. J. Adjoint-based optimization of a hypersonic inlet. In: AIAA. 22nd AIAA Computational Fluid Dynamics Conference. Dallas, TX, 2015.

KLINE, H. L.; ECONOMON, T. D.; ALONSO, J. J. Multi-objective optimization of a hypersonic inlet using generalized outflow boundary conditions in the continuous adjoint method. In: AIAA. 54th AIAA Aerospace Sciences Meeting. San Diego, CA, 2016.

KULFAN, B. M.; BUSSOLETTI, J. E. Fundamental parametric geometry representations for aircraft component shapes. In: 11th AIAA/ISSMO Multidisciplinary Analysis and Optimization Conference. Portsmouth, VA: [s.n.], 2006.

KURUVILA, G.; TAASAN, S.; SALAS, M. D. Airfoil Optimization by the OneShot Method, Optimum Design Methods in Aerodynamics. 1994. AGARD-FDPVKI Special Course.

LANEY, G. B. Computational Gasdynamics. New York, NY: Cambridge University Press, 1998.

LEVEQUE, R. J. Finite-Volume Methods for Hyperbolic Problems. [S.1.]: Cambridge University Press, 2002. (Cambridge Texts in Applied Mathematics).

LIGHTHILL, M. J. A new method of two-dimensional aerodynamics design. In: REM 2112, Aeronautical Research Council. [S.l.: s.n.], 1945.

LIONS, J. L. Optimal Control of Systems Governed by Partial Differential Equations. 1st. ed. Berlin: Spirnger-Verlag, 1971. (Die Grundelehren der mathematischen Wissenschaften, 170).

LOMAX, H.; PULLIAM, T. H.; ZINGG, D. W. Fundamentals of Computational Fluid Dynamics. [S.l.]: Springer-Verlag Berlin, 2001.

LUSTERNICK, L. A.; SOBOLEV, V. J. Elements of Functional Analysis. 1st. ed. Delhi: Hindustan Pub. Co., 1961. (Russian Monographs and Texts on Advanced Mathematics and Physics, V).

MAKINO, Y.; IWAMIYA, T.; LEI, Z. Fuselage shape optimization of a wingbody configuration with nacelles. Journal of Aircraft, v. 40, n. 2, p. 297-302, March-April 2003.

MALISKA, C. R. Transferência de Calor e Mecânica dos Fluidos Computacional. $2^{\mathrm{a}}$. ed. Rio de Janeiro, RJ: LTC - Livros Técnicos e Científicos Editora S.A., 2004 .

MANI, K.; MAVRIPLIS, D. Unsteady discrete adjoint formulation for twodimensional flow problems with deforming meshes. AIAA Journal, v. 46, n. 6, p. 1351-1364, June 2008.

MARCO, N.; BEUX, F. Multilevel Optimization: Application to One-Shot Shape Optimum Design. [S.l.], 1993. 
MARTINS, J. R. R. A.; ALONSO, J. J.; REUTHER, J. J. A coupled-adjoint sensitivity analysis method for high-fidelity aero-structural design. Optimization and Engineering, v. 6, p. 33-62, 2005.

MARTINS, J. R. R. A.; HWANG, J. T. Review and unification of discrete methods for computing derivatives of single- and multi-disciplinary computational models. AIAA Journal, 2013.

MCFADDEN, G. B. An Artificial Viscosity Method for the Design of Supercritical Airfoils. [S.l.], 1979.

MORSE, P. M.; FESHBACH, H. Methods of Theoretical Physics. 1st. ed. NY: MacGraw-Hill, 1953.

NADARAJAH, S.; JAMESON, A. Optimum shape design for unsteady threedimensional viscous flows using a non-linear frequency domain method. In: AMERICAN INSTITUTE OF AERONAUTICS AND ASTRONAUTICS. 24th Applied Aerodynamics Conference. San Francisco, CA: AIAA, 2006.

NADARAJAH, S. K.; JAMESON, A. Optimum shape design for unsteady flows with time-accurate continuous and discrete adjoint methods. AIAA Journal, v. 45 , n. 7 , p. $1478-1491$, July 2007.

NAIK, D. A.; KRIST, S. E.; CAMPBELL, R. L.; VATSA, V. N.; BUNING, P. G.; GEA, L. M. Inverse design of nacelles using multi-block Navier-Stokes codes. In: AMERICAN INSTITUTE OF AERONAUTICS AND ASTRONAUTICS. AIAA 33st Aerospace Sciences Meeting and Exhibit. Reno, NV: AIAA, 1995. AIAA 1995-1820.

NIELSEN, E. J.; LU, J.; PARK, M. A.; DARMOFAL, D. L. An implicit, exact dual adjoint solution method for turbulent flows on unstructured grids. Journal of Computers and Fluids, v. 33, n. 9, p. 1131-1155, November 2004.

OLIVEIRA, G. L.; TRAPP, L. G.; MACEDO, A. P. Engine-airframe integration methodologies for regional jet aircraft with underwing engines. In: AMERICAN INSTITUTE OF AERONAUTICS AND ASTRONAUTICS. AIAA 41st Aerospace Sciences Meeting and Exhibit. Reno, NV: AIAA, 2003. AIAA 2003-0934.

PALANIAPPAN, K.; SAHU, P.; JAMESON, A.; ALONSO, J. J. Design of adjoint-based laws for wing flutter control. Journal of Aircraft, v. 48, n. 1, p. 331-335, January-February 2011.

PIERCE, N.; GILES, M. Adjoint and defect error bounding and correction for functional estimates. Journal of Computational Physics, v. 200, p. 769-794, 2004.

PIRONNEAU, O. Optimal Shape Design for Elliptic Systems. New York: Springer-Verlag, 1983. (Springer Series in Computational Physics).

PRAGER, W. Introduction to Mechanics of Continua. [S.l.]: Ginn and Company, 1961.

REUTHER, J. J. Aerodynamic Shape Optimization Using Control Theory. Tese (Doutorado) — University of California Davis, 1996. 
REUTHER, J. J.; JAMESON, A. Control theory based airfoil design for potential flow and a finite volume discretization. In: AIAA. AIAA 32nd Aerospace Sciences Meeting and Exhibit. Reno, NV, 1994. AIAA Paper 94-0499.

REUTHER, J. J.; JAMESON, A. Aerodynamic shape optimization of wing and wing-body configurations using control theory. In: AIAA. 33rd Aerospace Sciences Meeting and Exhibit. Reno, NV, 1995. AIAA Paper 95-0123.

REUTHER, J. J.; JAMESON, A.; FARMER, J.; MARTINELLI, L.; SAUNDERS, D. Aerodynamic shape optimization of complex aircraft configurations via an adjoint formulation. In: AIAA. 34th Aerospace Sciences Meeting and Exhibit. Reno, NV, 1996. AIAA Paper 96-0094.

RODRIGUEZ, D. Response surface based optimization with a cartesian cfd method. In: AMERICAN INSTITUTE OF AERONAUTICS AND ASTRONAUTICS. AIAA 41st Aerospace Sciences Meeting and Exhibit. Reno, NV: AIAA, 2003. AIAA 2003-0465.

SADRI, R.; LEBLOND, D.; PIPERNI, P. Coupling of a wing inverse design code to an euler/navier-stokes flow solver using mesh movement capabilities. Advanced Aerodynamics, Bombardier Aerospace. 2002.

SANTOS, L. C. C. A Hybrid Inverse Optimization Method for Aerodynamic Design of Lifting Surfaces. Tese (Doutorado) - Georgia Institute of Technology, 1993.

SANTOS, L. C. C. A Study on Aerodynamic Design Optimization Using An Adjoint Method. Braunschweig, July 1995.

SCHWARTZ, L. Mathematics for the Physical Sciences. 1st. ed. Reading: Addison-Wesley, 1966. (Adiwes International Series in Mathematics).

TAASAN, S.; KURUVILA, G.; SALAS, M. D. Aerodynamic design and optimization in one shot. In: 30th Aerospace Sciences Meeting and Exhibit. Reno, NV: [s.n.], 1992. AIAA 92-0025.

THOMAS, J. P.; HALL, K. C.; DOWELL, E. H. Discrete adjoint approach for modeling unsteady aerodynamic design sensitivities. AIAA Journal, v. 43, n. 9, p. 1931-1936, September 2005.

VANDERPLAATS, G. N. Numerical Optimization Techniques for Engineering Design: With Applications. 1st. ed. N.Y.: McGraw-Hill, 1984. (Series in Mechanical Engineering).

VENDITTI, D. A.; DARMOFAL, D. L. A multilevel error estimation and grid adaptive strategy for improving the accuracy of integral outputs. 1999. AIAA Paper 99-3292.

VENDITTI, D. A.; DARMOFAL, D. L. Adjoint error estimation and grid adaptation for functional outputs: Application to quasi-one-dimensional flow. Journal of Computational Physics, v. 164, p. 204-227, 2000.

VENDITTI, D. A.; DARMOFAL, D. L. Grid adaptation for functional outputs: Application to two-dimensional inviscid flows. Journal of Computational Physics, v. 176, p. 40-69, 2002. 
VENDITTI, D. A.; DARMOFAL, D. L. Anisotropic grid adaptation for functional outputs: application to two-dimensional viscous flows. Journal of Computational Physics, v. 187, p. 22-46, 2003.

VOLPE, E. V. A11 - Inverse Aerodynamic Design Module. [S.1.], 2004.

VOLPE, E. V. A3 - Inverse Aerodynamic Design Applications. [S.1.], 2005.

VOLPE, E. V. Análise conceitual de projeto inverso e otimização aerodinâmica com foco no método adjunto. Tese de Livre Docência — EPUSP. 2011.

VOLPE, E. V.; OLIVEIRA, G. L.; SANTOS, L. C. C.; HAYASHI, M. T.; CEZE, M. A. B. Inverse aerodynamic design applications usign the mgm hybrid formulation. In: Inverse Problems Design and Optimization Symposium. Miami, FL: [s.n.], 2007.

VOLPE, E. V.; SANTOS, L. C. C. Boundary and internal conditions for adjoint fluid flow problems - applications to quasi-1d euler equations. Journal of Engineering Mathematics (paper accepted for publication). 2009.

ZINGG, D. W.; NEMEC, M.; PULLIAM, T. H. A comparative evaluation of genetic and gradient-based algorithms applied to aerodynamic optimization. European Journal of Computational Mechanics, v. 17, n. 1-2, p. 103-126, 2008. 


\section{ApÊNDICE A - COEFICIENTES DAS EquaÇões Características AdJuntas}

A tabela A.1 apresentada a seguir mostra os valores atribuídos aos coeficientes

$K_{\alpha \beta}^{t}, K_{\alpha \beta}^{x}$ e $K_{\alpha \beta}^{y}$ das equações características adjuntas bidimensionais (3.25). Os índices dos coeficientes são definidos da seguinte maneira: $\alpha$ indica a equação do sistema característico; $\beta$, refere-se à variável adjunta a que está multiplicando; enquanto o superescrito $t, x$ ou $y$ indica ao tipo de derivada que está associada (temporal, espacial na direção coordenada x ou y, respectivamente).

Aqui, vale ressaltar que todos os coeficientes são constantes no tempo durante a solução da equação adjunta em cada elemento da malha. Isto ocorre porque, nas aplicações de interesse desta Tese, a solução adjunta é obtida para uma solução estacionária do escoamento. Mais do que isso, como eles não dependem das variáveis adjuntas ou suas derivadas, caracterizam as PDE's adjuntas como lineares. 
Tabela A.1: Coeficientes das equações características adjuntas (3.25)

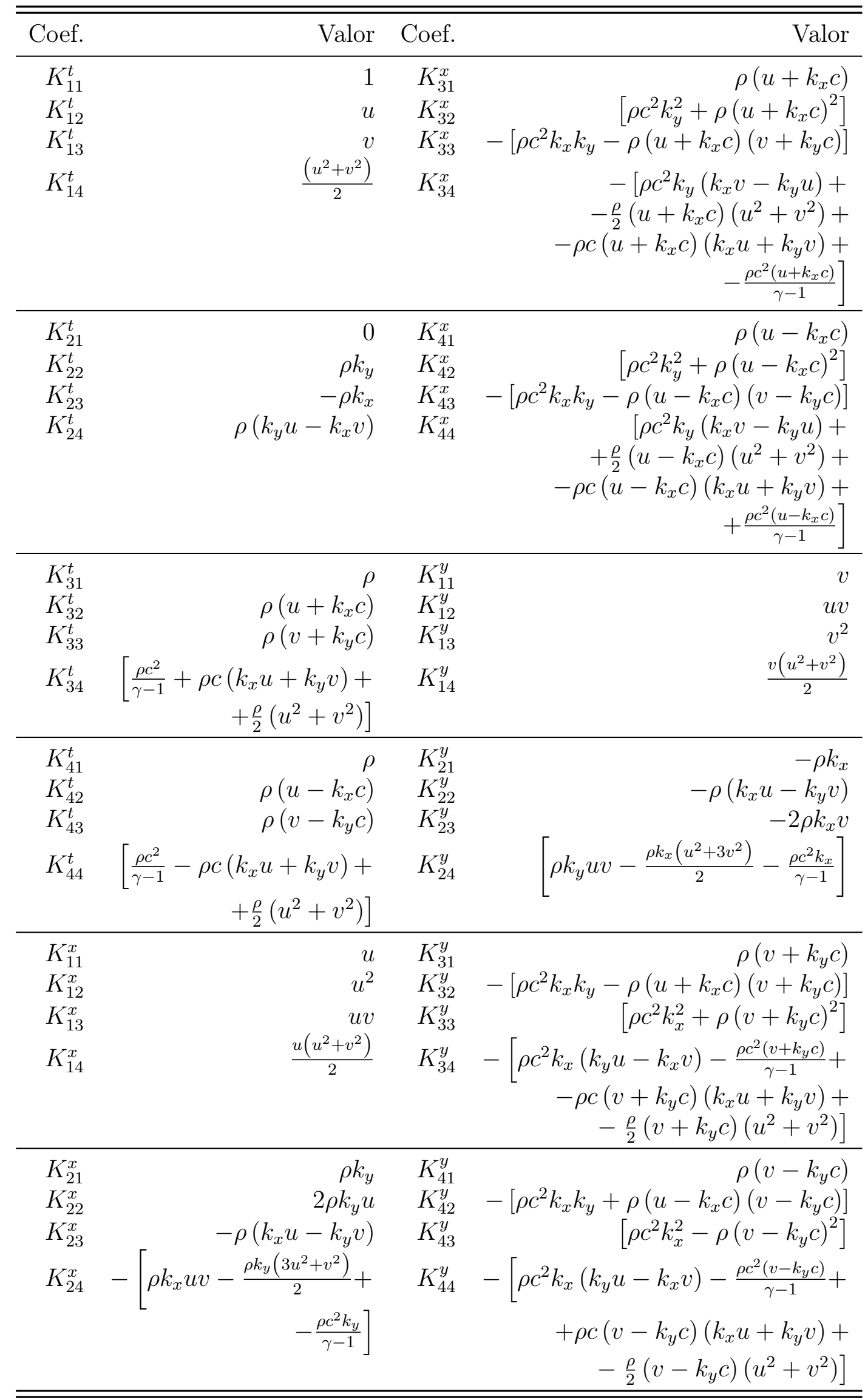




\section{ApÊndiCE B - REDUÇÃo Do GRAdiente GEOMÉTRICO}

Os termos $e, g$ e $h$ do gradiente de sensibilidade dado pela eq. (3.14), que apenas tratam de variações geométricas podem ser ainda mais simplificados. Como cada variação de parâmetro é tomada como independente das demais, qualquer variação puramente geométrica é considerada na ausência de qualquer outra mudança de parâmetros. Portanto, esses termos podem estar sujeitos às mesmas simplificações que foram propostas por Jameson e Kim (2003b). Sob essas condições, a eq. (3.14) pode ser reduzida a:

$$
\begin{aligned}
& \delta G=\underbrace{\left\langle\delta \phi_{\alpha}, \frac{\partial F_{\alpha}^{k}}{\partial \xi^{k}}\right\rangle+\langle\delta \psi, \mathcal{B}\rangle_{s_{i}}+\left\langle\delta \mathbf{a}, \alpha-\alpha_{o}\right\rangle}_{(a)}-\underbrace{\left\langle\frac{\left.C_{\alpha \beta}^{k} \frac{\partial\left(J \phi_{\alpha}\right)}{J \xi^{k}}, \delta \bar{Q}_{\beta}\right\rangle}{J}\right\rangle}_{(b)}+ \\
&+\underbrace{\left\langle\psi_{\alpha} \frac{\partial \mathcal{B}_{\alpha}}{\partial Q_{\beta}}+\phi_{\alpha} C_{\alpha \beta}^{2} n_{2}, \delta \bar{Q}_{\beta}\right\rangle_{s_{i}}}_{(d)}+\underbrace{\left\langle\phi_{\alpha} C_{\alpha \beta}^{2} n_{2}, \delta \bar{Q}_{\beta}\right\rangle_{s_{o}}}_{(f)}+ \\
&+ \underbrace{\left\langle\frac{\partial \mathcal{F}}{\partial Q_{\alpha}}\left|\frac{\mathrm{d} S^{\prime}}{\mathrm{d} S}\right|+\left[\phi_{\left(i^{\prime}+1\right)} J \beta_{i^{\prime}}^{2} n_{2}\right] \frac{\partial p}{\partial Q_{\alpha}}, \delta Q_{\alpha}\right\rangle_{b_{w}}}_{(j)}+\underbrace{\langle\mathbf{a}, \delta \alpha\rangle+\left\langle\psi, \mathcal{B}_{\alpha}^{\prime} \delta \alpha\right\rangle_{s_{i}}}_{(i)}+ \\
& \underbrace{}_{b_{w}\left\{\phi_{\alpha}\left[\delta\left(J \beta_{i^{\prime}}^{2}\right) f_{\alpha}^{i^{\prime}}+C_{\alpha \beta}^{2} \delta Q_{\beta}^{*}\right] n_{2}+\mathcal{F} \delta\left|\frac{\mathrm{d} S^{\prime}}{\mathrm{d} S}\right|+p \phi_{\left(i^{\prime}+1\right)} \delta\left(J \beta_{i^{\prime}}^{2}\right) n_{2}\right\}} \mathrm{d} S
\end{aligned}
$$

O procedimento substitui os três termos $e, g$ e $h$ por uma única integral sobre $b_{w}$, que agora é chamada $j$. Além disso, duas novas variações são trazidas à tona na eq. (B.1). São elas: $\delta Q_{\beta}^{*}$ e $\delta \bar{Q}_{\beta}=\delta Q_{\beta}-\delta Q_{\beta}^{*}$. A primeira, representa variações nas variáveis de estado que são devidas apenas ao movimento da malha a uma configuração de contorno fixa (JAMESON; KIM, 2003b). Apesar da sua causa particular, $\delta \xi^{k}$, elas são da mesma natureza das variações originais $\delta Q_{\beta}$, de tal modo que devem satisfazer uma equação que é totalmente análoga à de Euler. 


\section{APÊNDICE C - Diagrama $\boldsymbol{\theta}-\boldsymbol{\beta}-\boldsymbol{M}$}

Usando a equação da continuidade e o fato de que a componente tangencial da velocidade não muda ao atravessar o choque, algumas relações trigonométricas levam a uma equação que concatena o ângulo de deflexão $\theta$ da gemetria, o ângulo do choque $\beta$ e o número de Mach $M_{1}$ a montante do choque (ANDERSON, 2011):

$$
\operatorname{tg} \theta=2 \operatorname{cotg} \beta \frac{M_{1}^{2} \operatorname{sen}^{2} \beta-1}{M_{1}^{2}[\gamma+\cos (2 \beta)]+2}
$$

Esta função chamada relação $\theta-\beta-M$ especifica $\theta$ como função exclusivamente de $M_{1}$ e $\beta$. A figura C.1 mostra justamente os resultados dessa equação plotados em um gráfico. Ela é, particularmente, utilizada nesta Tese para verificar se as soluções em regime de escoamento supersônico ao redor de um perfil diamante (seção 4.4.1) apresentam ondas de choque destacadas ou não. Para fazer essa análise, basta considerar a curva de nível de um dado número de Mach $M_{1}$, representado pelas linhas azuis na figura C.1 e verificar o ponto de cruzamento com a linha vermelha, que representa $\theta_{\max }$ para que exista uma onda de choque oblíqua colada na superfície sólida. Caso a geometria seja tal que seu ângulo de deflexão $\theta>\theta_{\max }$ a natureza estabelecerá um choque curvo destacado da parede (ANDERSON, 2011). 


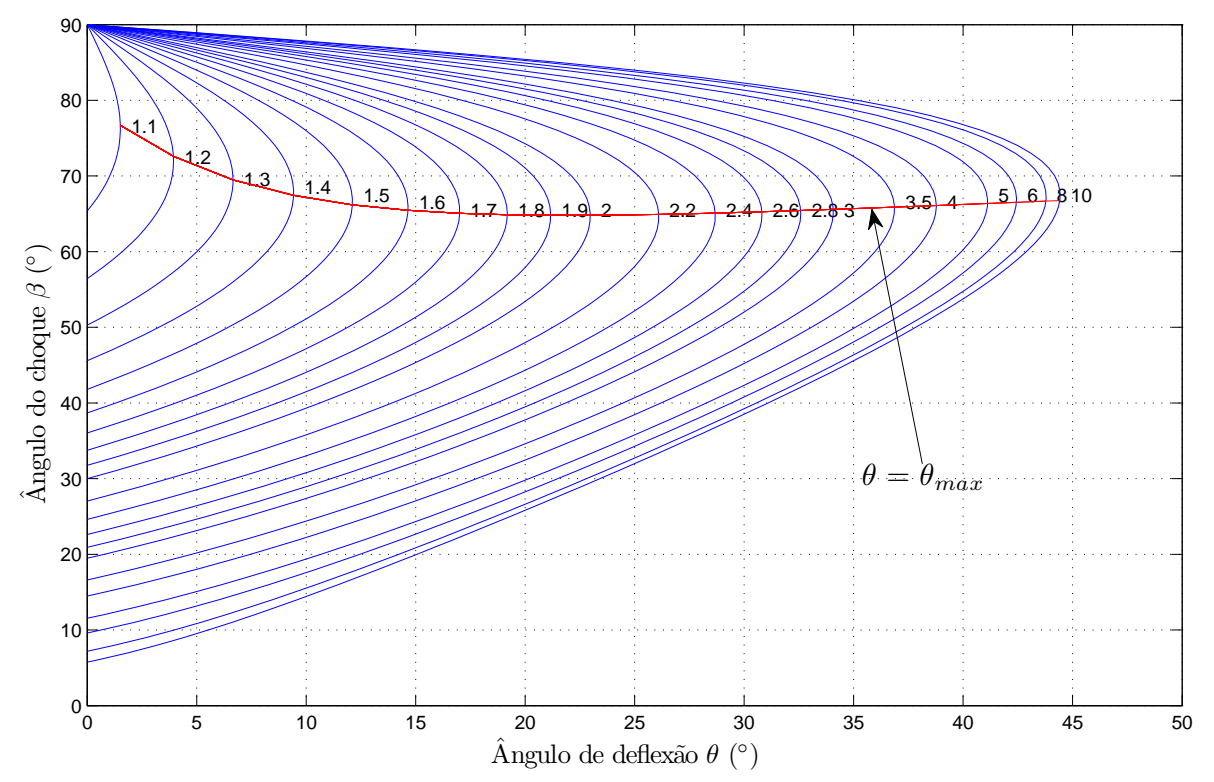

Figura C.1: Diagrama $\theta-\beta-M$ 